\title{
VITAMIN D, INFLAMMATION AND THEIR INTERPLAY IN COLORECTAL CANCER PROGNOSIS
}

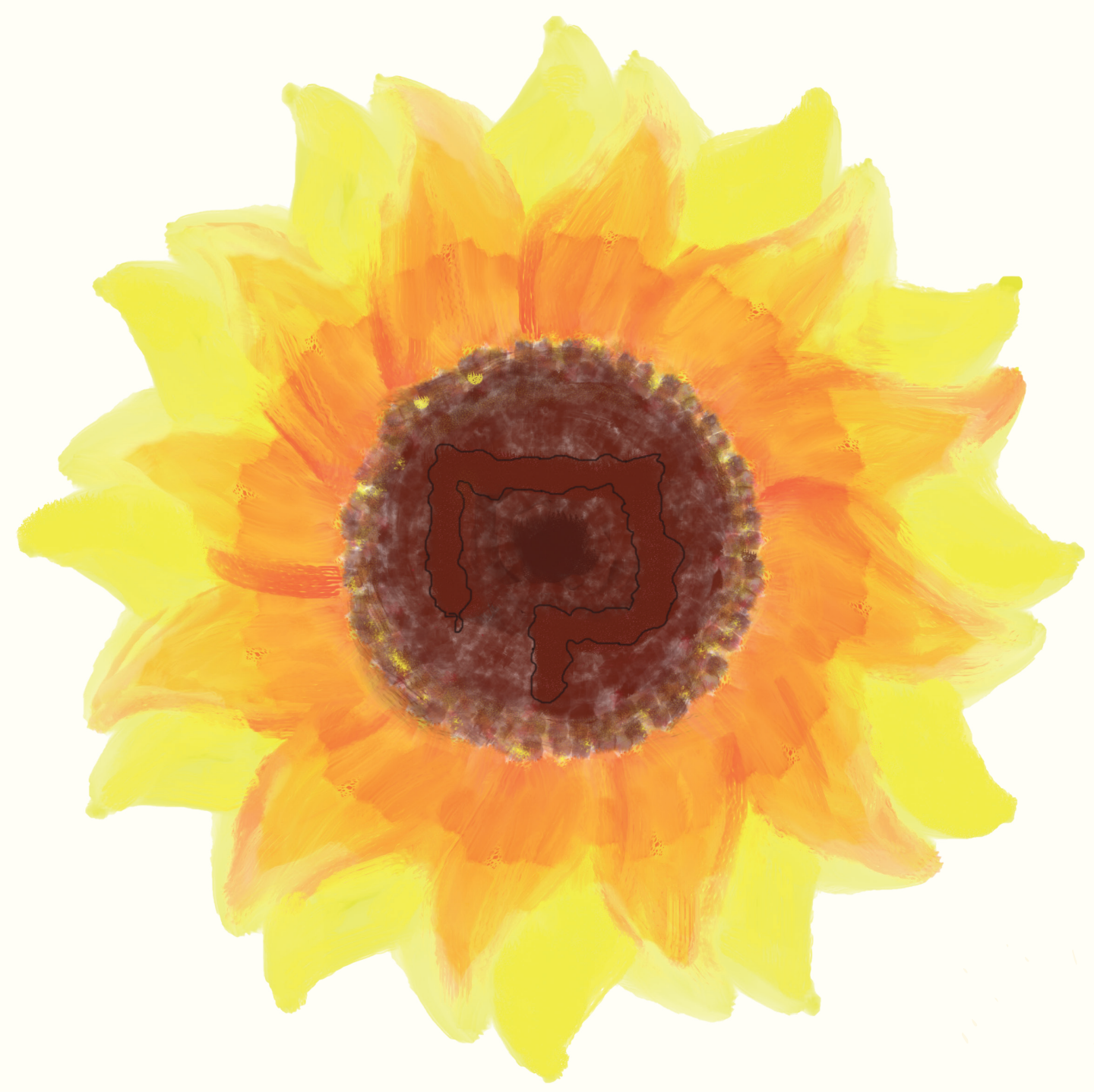

Evertine Wesselink 



\section{Propositions}

1. Higher vitamin D concentrations are associated with a lower risk of total mortality in colorectal cancer patients, especially in combination with a high magnesium intake.

(this thesis)

2. A dose of vitamin $D$ a day lowers inflammation, but does not keep it away. (this thesis)

3. A molecular pathological epidemiology research approach is necessary to unravel how nutrition is related to the pathogenesis of neoplastic and non-neoplastic diseases.

4. The cause of a nutrient deficiency in an individual must be explored rather than blindly giving high dose supplements to resolve the deficiency.

5. Data waste, i.e. inefficient use of collected data, is a major problem in the academic world.

6. Instead of protecting individuals, the General Data Protection Regulation of 2018 harms individuals.

7. Circular agriculture is the best form of sustainable farming.

Propositions belonging to the thesis, entitled:

"Vitamin D, inflammation and their interplay in colorectal cancer prognosis"

Evertine Wesselink

Wageningen, 12 October 2021 



\section{Vitamin D, inflammation and their interplay in colorectal cancer prognosis}

Evertine Wesselink 


\section{Thesis committee}

\section{Promotor}

Prof. Dr Ellen Kampman

Professor of Nutrition and Disease

Wageningen University \& Research

\section{Co-promotors}

Dr Fränzel J.B. van Duijnhoven

Assistant Professor, Nutrition, Genes and Colorectal cancer

Wageningen University \& Research

Dr Martijn J.L. Bours

Assistant Professor of Epidemiology

Maastricht University

\section{Other members}

Prof. Dr Edward L. Giovannucci, Harvard T.H. Chan School of Public Health, Boston, USA Prof. Dr Lisette C.P.G.M. de Groot, Wageningen University \& Research

Prof. Dr Miriam Koopman, University Medical Center Utrecht

Prof. Dr Huub F.J. Savelkoul, Wageningen University \& Research

This thesis was conducted under auspices of the Graduate School VLAG (Advanced Studies in Food Technology, Agrobiotechnology, Nutrition and Health Sciences). 


\title{
Vitamin D, inflammation and their interplay in colorectal cancer prognosis
}

\author{
Evertine Wesselink
}

\section{Thesis}

submitted in fulfilment of the requirements for the degree of doctor

at Wageningen University

by the authority of the Rector Magnificus,

Prof. Dr A.P.J. Mol,

in the presence of the

Thesis Committee appointed by the Academic Board

to be defended in public

on Tuesday 12 October 2021

at 4 p.m. in the Aula. 
Evertine Wesselink

Vitamin D, inflammation and their interplay in colorectal cancer prognosis, 240 pages.

PhD thesis, Wageningen University, Wageningen, the Netherlands (2021)

With references, with summary in English and Dutch

ISBN: 978-94-6395-861-5

DOI: https://doi.org/10.18174/548920 


\section{TABLE OF CONTENT}

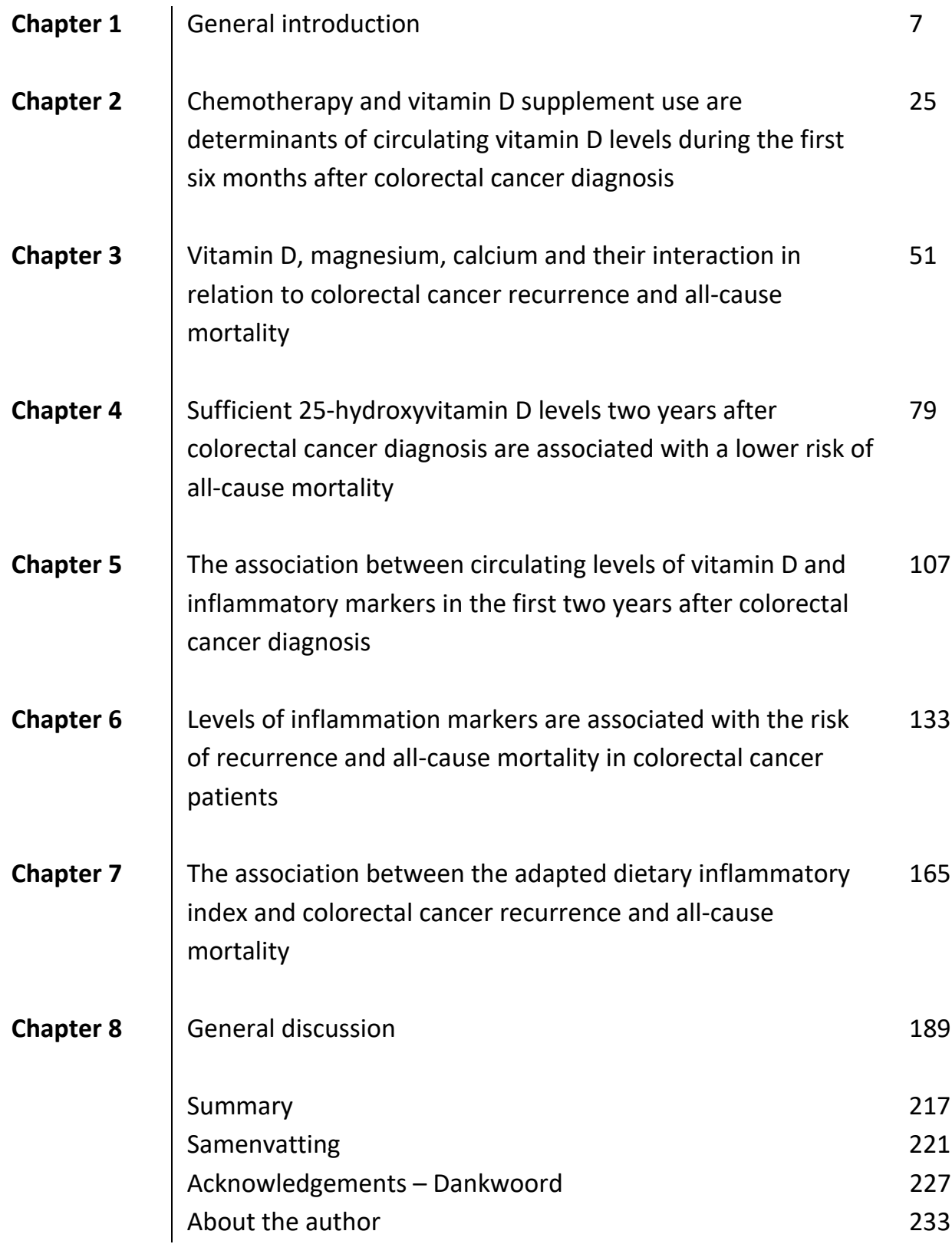




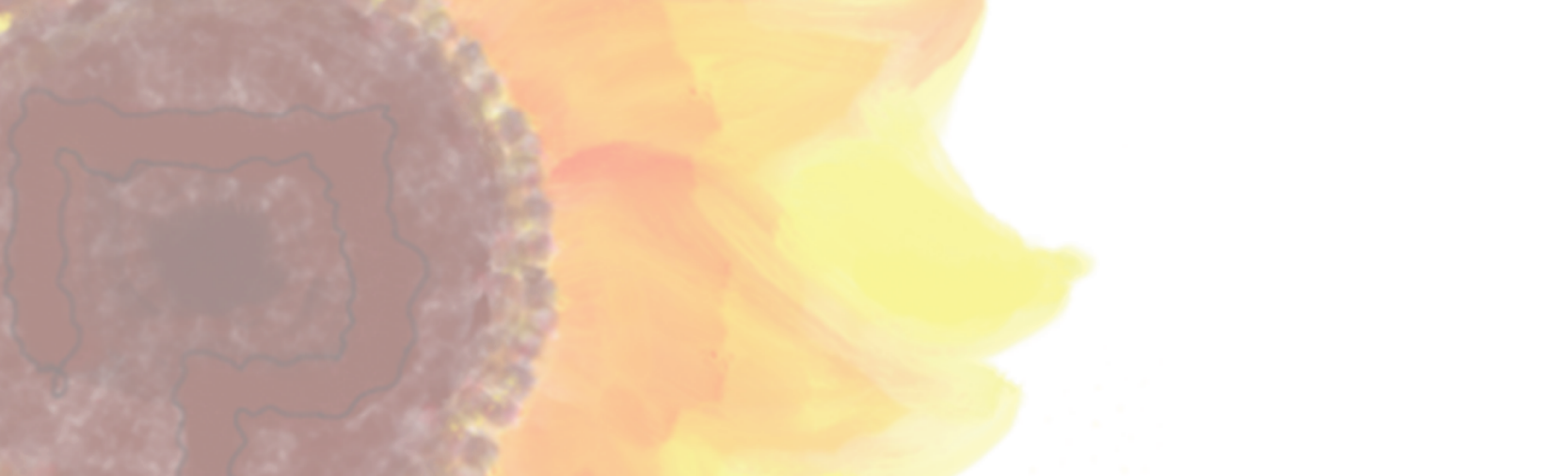


CHAPTER 1

GENERAL INTRODUCTION 

Colorectal cancer (CRC) accounts for one in ten cancer cases and deaths worldwide and the incidence of CRC is still increasing [1]. The number of CRC survivors is further increasing due to population screening programs and better treatments [2]. However, many CRC survivors suffer from side-effects of treatment, such as fatigue, neuropathy and an increased risk of cardiometabolic diseases, which may impact quality of life and prognosis [3-5]. Cancer recurrence is another major concern for CRC survivors and an important contributor to mortality in this growing population [6, 7]. Therefore, it is necessary to optimize long-term survival in CRC patients. Vitamin D is a potentially interesting compound in this respect as associations of vitamin D with a lower CRC risk and reduced risk of total cancer mortality have been shown in the general population [8, 9]. In addition, high compared to low concentrations of vitamin D at diagnosis are associated with higher survival rates in CRC survivors [10]. It is hypothesized that vitamin D exerts its beneficial effect on CRC outcomes due to its anti-inflammatory potential [11]. Nevertheless, until now many questions remain unanswered regarding the "black box" between vitamin D and CRC prognosis. For example, is vitamin D deficiency causally related to worse CRC prognosis? Does vitamin D improve CRC prognosis by reducing inflammation? Are vitamin D and inflammation associated with CRC recurrences?

\section{VITAMIN D DEFICIENCY}

Vitamin D deficiency is common in CRC patients [12-16] and is associated with adverse health outcomes and a higher risk of mortality [17]. Serum twenty-five hydroxy vitamin $D_{3}$ $\left(25(\mathrm{OH}) \mathrm{D}_{3}\right)$ is the main circulating form of vitamin $\mathrm{D}$ and generally accepted as a robust and reliable biomarker of vitamin D status [18]. As much as $40 \%$ of the European population is vitamin D deficient (serum $25(\mathrm{OH}) \mathrm{D}_{3}<50 \mathrm{nmol} / \mathrm{L}$ ) [19]. In CRC patients the prevalence of vitamin D deficiency is even higher, ranging between 40 and $87 \%$ at the time of diagnosis [12-16]. Low serum $25(\mathrm{OH}) \mathrm{D}_{3}$ concentrations are associated with many adverse health outcomes. First of all, the lowest category of $25(\mathrm{OH}) \mathrm{D}_{3}$ concentrations compared to highest category of $25(\mathrm{OH}) \mathrm{D}_{3}$ concentrations is associated with a higher risk of CRC-specific and all-cause mortality [10]. Secondly, vitamin D deficiency is associated with a higher prevalence of osteoporosis, depression and fatigue in the general population [20-22]. Those adverse health outcomes are often reported by CRC survivors, even years after diagnosis and are seriously affecting their quality of life and daily functioning [23-26]. The high prevalence of vitamin D deficiency in CRC patients, together with the possible beneficial effects of $25(\mathrm{OH}) \mathrm{D}_{3}$ on quality of life, cancer progression and survival warrants further study on determinants of serum $25(\mathrm{OH}) \mathrm{D}_{3}$ concentrations and changes in $25(\mathrm{OH}) \mathrm{D}_{3}$ concentrations over time during and after cancer treatment. Knowing which determinants are associated with low $25(\mathrm{OH}) \mathrm{D}_{3}$ concentrations will enable health professionals to identify and monitor patients at high risk of a vitamin D deficiency during and after treatment. 


\section{DETERMINANTS OF VITAMIN D STATUS}

Sunlight, some food products and vitamin D supplements are sources of vitamin $D$, of which sunlight is the most important one [27]. The ability to synthesize vitamin D in the skin is dependent on age, skin pigmentation, clothing style, season and the use of sunblock [27]. Food products rich in vitamin D are fatty fish, fortified margarine and dairy products, eggs and mushrooms [28]. Serum $25(\mathrm{OH})_{3}$ concentrations are also influenced by physical activity, body mass index (BMI) and other dietary factors. Higher physical activity levels and dietary calcium intake are associated with higher $25(\mathrm{OH}) \mathrm{D}_{3}$ concentrations, while a higher $\mathrm{BMI}$ and an older age are associated with lower $25(\mathrm{OH}) \mathrm{D}_{3}$ concentrations in healthy adults [27, 29-32].

Next to these demographic and lifestyle factors, also the cancer itself and cancer treatment may influence vitamin D concentrations. Very low $25(\mathrm{OH}) \mathrm{D}_{3}$ levels (median 17 $25 \mathrm{nmol} / \mathrm{L}$ ) were observed in $\mathrm{CRC}$ patient cohorts in which $25(\mathrm{OH}) \mathrm{D}_{3}$ concentrations were measured shortly after surgery $[12,14,15]$, while $25(\mathrm{OH}) \mathrm{D}_{3}$ concentrations ranging between 43 and $65 \mathrm{nmol} / \mathrm{L}$ were observed in cohorts in which concentrations were measured before surgery [13, 16, 33-37]. Moreover, in a small study in 92 CRC patients a post-operative decline in $25(\mathrm{OH}) \mathrm{D}_{3}$ concentrations followed by a recovery to above preoperative concentrations was observed [38]. Next to that, results of previous studies in patients receiving chemotherapy, showed either a higher risk of having vitamin $D$ deficiency [39] or a decrease in $25(\mathrm{OH}) \mathrm{D}_{3}$ concentrations over time [40]. However, the relative importance of dietary and other lifestyle factors for vitamin $D$ levels in conjunction with clinical factors in CRC patients is presently unknown.

\section{INTERACTION WITH CALCIUM AND MAGNESIUM}

Other nutrients, such as calcium and magnesium, may influence vitamin D metabolism and activation [41]. Vitamin $D_{3}$ obtained from sunlight exposure, foods or supplements must undergo two activation steps before it is converted to its biologically active form calcitriol $\left(1,25(\mathrm{OH})^{2} \mathrm{D}_{3}\right)$ (Figure 1). Vitamin $\mathrm{D}$, calcium and magnesium already interact on the absorption level in the intestine [41]. Higher $25(\mathrm{OH}) \mathrm{D}_{3}$ concentrations stimulate calcium absorption and higher calcium concentrations results in a longer half life time for $25(\mathrm{OH}) \mathrm{D}_{3}$, implying a vitamin D sparing effect [42]. On the other hand, higher calcium intake can exacerbate the onset of a low magnesium status and vice versa [41]. After absorption into the blood stream, vitamin $\mathrm{D}$ is transported by vitamin $\mathrm{D}$ binding protein (VDBP). The production of VDBP is magnesium-dependent. In addition, activities of three major enzymes involved in vitamin D metabolism: 25-hydroxylase, $1 \alpha$-hydroxylase and 24hydroxylase, are magnesium dependent. Therefore, magnesium deficiency can lead to an inadequate vitamin D activation $[43,44]$. 
Given the role of magnesium and calcium in several biochemical processes involved in the synthesis and metabolism of vitamin D (Figure 1), magnesium and/or calcium intake could modify the association between vitamin $\mathrm{D}$ and $\mathrm{CRC}$ outcomes. A previous cohort study in the general population observed a stronger inverse association between $25(\mathrm{OH}) \mathrm{D}_{3}$ concentrations and all-cause mortality in participants with a high magnesium intake

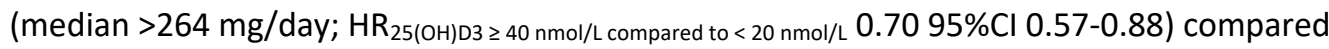

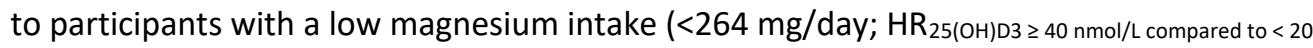
$\mathrm{nmol} / \mathrm{L} 0.8795 \% \mathrm{Cl} 0.64-1.20$ ) [44]. Although vitamin $\mathrm{D}$ and calcium in relation to CRC survival have been studied together $[45,46]$, the interaction between calcium and vitamin $D$ in relation to CRC outcomes has not yet been studied. Whether magnesium and/or calcium, alone or in interaction with vitamin $D$, are beneficial for patients with $C R C$ is therefore unknown.

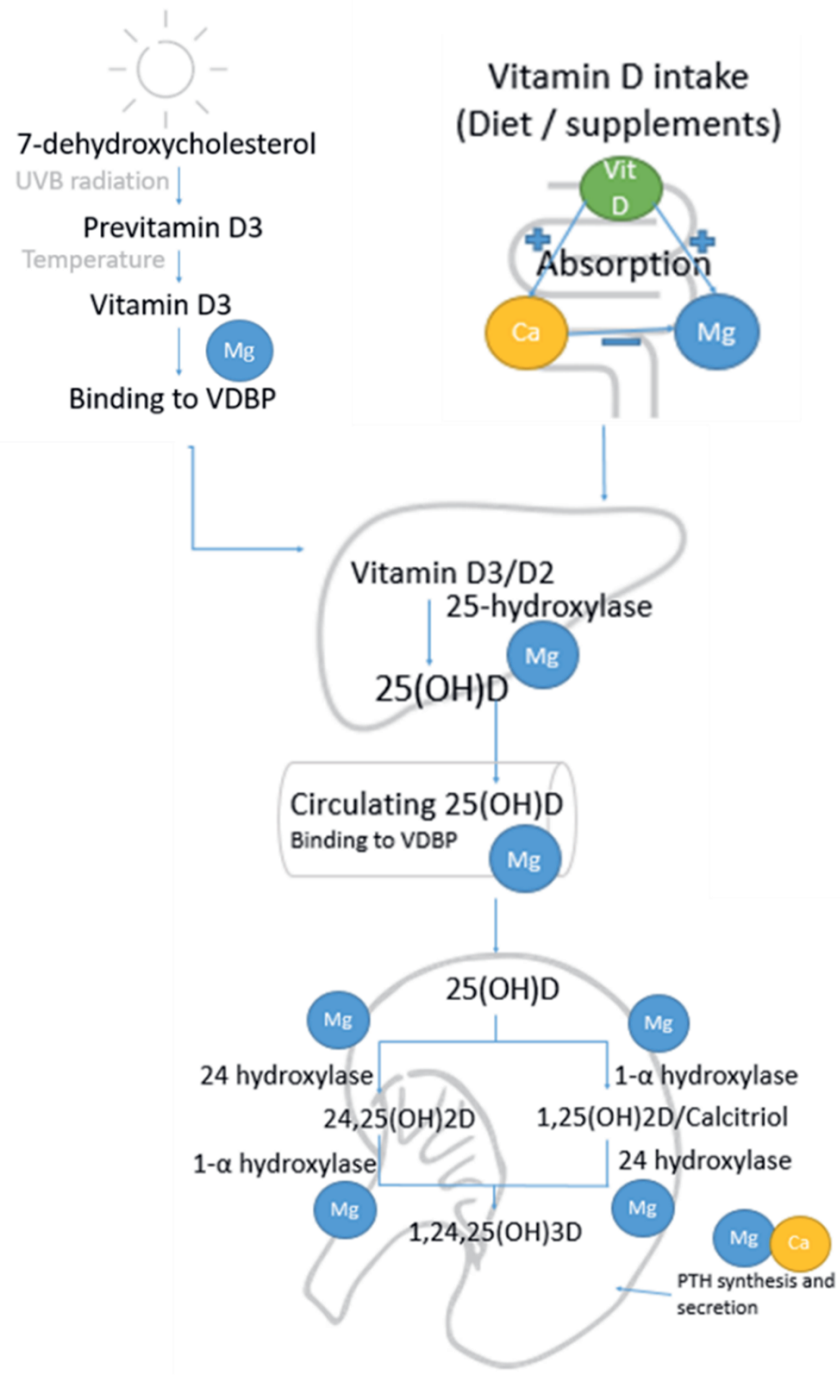


Figure 1: Vitamin D metabolism and interaction between vitamin $D$, magnesium and calcium; adapted from Deng 2013. Vitamin D, calcium and magnesium interact on the absorption level in the intestine, where vitamin D stimulates the absorption of calcium and magnesium, while higher calcium intake hampers the absorption of magnesium.

Magnesium is an essential cofactor for 3 major enzymes involved in vitamin D metabolism. VDBP: vitamin D binding protein; Mg: magnesium; Ca: calcium; 25(OH)D: 25-hydroxy vitamin $D ; 1,25(O H)^{2} D: 1,25$ dihydroxy vitamin $D$;

\section{VITAMIN D AND COLORECTAL CANCER OUTCOMES}

Evidence is accumulating that circulating vitamin D concentrations are also inversely associated with mortality in CRC patients [12, 14-16, 33-35, 47-50]. Wu and colleagues performed a meta-analysis in 2020, including 17 original cohort studies in CRC patients with a total of 17,770 participants [10]. Pooled effect estimates comparing the highest versus lowest category of circulating $25(\mathrm{OH}) \mathrm{D}_{3}$ at or shortly after diagnosis showed a hazard ratio $(\mathrm{HR})$ of 0.64 (95\% confidence interval $(\mathrm{Cl})$ 0.55-0.72) for all-cause mortality and $0.65(95 \% \mathrm{Cl} 0.56-0.73)$ for CRC-specific mortality. Wu and colleagues also conducted dose-response analyses in which a risk reduction of $7 \%(\mathrm{HR} 0.9395 \% \mathrm{Cl} 0.90-95)$ for allcause mortality and $12 \%$ (HR $0.8895 \% \mathrm{Cl} 0.84-0.93$ ) for cancer-specific mortality was observed for each $20 \mathrm{nmol} / \mathrm{L}$ increment in $25(\mathrm{OH}) \mathrm{D}_{3}$ concentration [10]. The association between $25(\mathrm{OH}) \mathrm{D}_{3}$ concentrations and $\mathrm{CRC}$ recurrence is hardly reported so far. One previous study including 2,910 patients diagnosed with CRC observed a $32 \%(95 \% \mathrm{Cl} 1.03-$ $1.71 \%)$ higher risk of recurrence in patients with very low plasma $25(\mathrm{OH}) \mathrm{D}_{3}$ concentrations at diagnosis $\left(<11.8 \mathrm{nmol} / \mathrm{L}\right.$ ) compared to patients with $25(\mathrm{OH}) \mathrm{D}_{3}$ concentrations $>45.2$ $\mathrm{nmol} / \mathrm{L}$, independent of stage of disease [15].

Until now, previous studies measured $25(\mathrm{OH}) \mathrm{D}_{3}$ concentrations at one moment in time. Measuring $25(\mathrm{OH}) \mathrm{D}_{3}$ concentrations several times during and after treatment could provide more insights into the causality of the longitudinal relationship between (changes in) $25(\mathrm{OH}) \mathrm{D}_{3}$ concentrations and $\mathrm{CRC}$ outcomes. Only one previous study investigated timing of vitamin D measurements in relation to CRC outcomes. In this study, combining data of in total 3,535 CRC patients higher versus lower pre-operative as well as higher versus lower post-operative $25(\mathrm{OH}) \mathrm{D}_{3}$ concentrations were associated with a lower risk of all-cause mortality (HR $0.3095 \% \mathrm{Cl} 0.12-0.71$; $\mathrm{HR} 0.6595 \% \mathrm{Cl} 0.51-0.81$, respectively) [38]. Although timing of $25(\mathrm{OH}) \mathrm{D}_{3}$ measurements (pre versus postoperative) was investigated in the study, only one measurement per patient was taken, while $25(\mathrm{OH}) \mathrm{D}_{3}$ concentrations are likely to change over time within individuals $[39,51]$. Such changes over time, for example a steep decrease during cancer treatment or delayed recovery of $25(\mathrm{OH}) \mathrm{D}_{3}$ concentrations after treatment, could also affect health outcomes in CRC patients. Until now, however, changes in $25(\mathrm{OH}) \mathrm{D}_{3}$ concentrations during and after treatment in relation to CRC outcomes have not been investigated. 


\section{VITAMIN D, INFLAMMATION AND COLORECTAL CANCER PROGRESSION}

Vitamin D is hypothesized to influence cancer progression via several mechanisms as it can stimulate cell differentiation and inhibit angiogenesis, cell migration and metastases [5255]. Modulation of inflammatory processes is considered to be one of the major mechanisms by which vitamin $D$ exerts its beneficial effects on tumour progression [11, 54, 56-62]. Results of in-vitro and animal studies showed that calcitriol can affect multiple inflammatory processes involved in CRC progression, including the Nuclear Factor kappa B (NFKB) pathway [56-59] (Figure 2). Calcitriol inhibits the activity of NFKB, which is a key regulator of immune responses and inflammation. When stimulated, NFKB activates the transcription of various genes involved in pro-inflammatory and pro-tumorigenic processes $[60,61]$. Inhibiting NFKB thereby results in the release of less pro-inflammatory mediators $[54,58]$. Another pathway by which calcitriol can affect inflammatory processes involved in CRC progression is by increasing the expression of MAP kinase phosphatase 5 (MKP5). The increased expression of MKP5 leads to downstream anti-inflammatory processes by dephosphorylation and inactivation of p38 stress-induced kinases. In turn, this results in a decreased production of pro-inflammatory cytokines that sustain and amplify inflammatory responses such as interleukin-6 (IL6) and tumour necrosis factor- $\alpha$ $(\mathrm{TNF} \alpha)[11,59]$.

Although the role of vitamin D in inflammation has extensively been studied in in-vitro and animal studies, studies in humans remain limited. Results of a recent study in CRC patients showed a weak correlation between $25(\mathrm{OH}) \mathrm{D}_{3}$ concentrations and C-reactive protein (CRP) concentrations before and after surgery [38]. In addition, one clinical trial in colorectal adenoma patients observed a non-statistically significant decline in CRP, IL6, IL1ß and TNF $\alpha$ concentrations after supplementation with $20 \mu \mathrm{g} /$ day of vitamin $D_{3}$ for 6 months. Moreover, a combined inflammatory z-score decreased with $77 \%$ in the vitamin D group compared with a $33 \%$ decrease in the control group [63]. Such a combined inflammatory z-score is often calculated to cluster conceptually related markers of lowgrade inflammation and to improve statistical efficiency [64]. To the best of my knowledge, no other studies assessed the relation between vitamin $D$ and inflammation in CRC patients. Therefore, in this thesis we will investigate circulating inflammation markers potentially influenced by vitamin D and associated with CRC progression, including IL6, IL8, IL10, TNF $\alpha$ [57]. Investigating inflammation as one of the potential underlying mechanisms between vitamin $\mathrm{D}$ and CRC outcomes will provide more evidence for a causal inference regarding the relationship between vitamin D concentrations and CRC outcomes. 


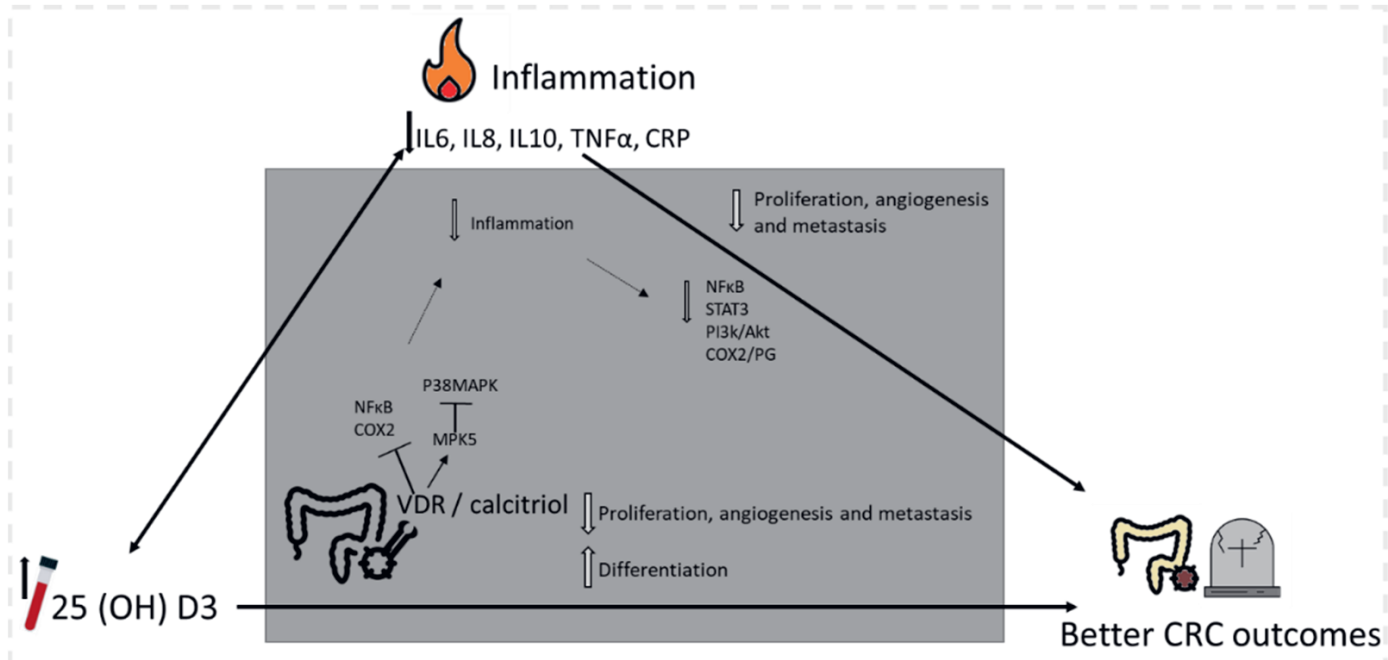

Figure 2: Overview of associations between circulating $25(\mathrm{OH}) \mathrm{D}_{3}$ concentrations, inflammation and CRC outcomes investigated in this thesis and not yet investigated ("black box"), but suggested underlying mechanisms.

\section{INFLAMMATION AND COLORECTAL CANCER OUTCOMES}

Inflammation plays an important role in tumour promotion [65] and circulating inflammation markers are associated with worse CRC outcomes [66-72]. Pro-inflammatory cytokines can serve as direct growth factors [65]. Besides, inflammatory factors are essential in shaping cell plasticity within the tumour microenvironment, which further affects tumour growth [65]. NFKB-dependent cytokines, such as IL6, TNF $\alpha$ and IL8, induce tumour growth through the activation of oncogenic pathways including signal transducer and activator of transcription 3 (STAT3). Furthermore, inflammatory cytokines also stimulate angiogenesis and alter mechanical and metabolic properties of stromal and tumour cells [65]. Finally, inflammation also influences cancer invasion, epithelial to mesenchymal transition and cell migration $[65,73,74]$. Inflammation is hypothesized to be even more important in tumour metastasis and cancer mortality than in cancer initiation [65].

CRP is a frequently measured inflammatory marker in epidemiological studies, because it is relatively easy and cheap to measure. CRP plays important roles in inflammatory processes, apoptosis and the production of cytokines, particularly IL6 and TNF $\alpha$ [75], which in turn stimulates CRC progression. However, CRP is a non-specific marker of inflammation. Assessing inflammation markers that are suggested to be involved in tumour progression such as IL6, IL8 and TNF $\alpha[61,76]$, could provide more insights into the relationship between specific inflammatory pathways and tumour progression in humans. 
Higher concentrations of pro-inflammatory markers, including IL6, TNF $\alpha$, CRP and IL8, have been associated with more advanced CRC in small studies in CRC patients [66, 69, 7779]. Higher concentrations of IL6, IL8, IL10 and TNF $\alpha$ have also been associated with a worse disease free-, cancer specific- and overall survival [66-72]. Higher levels of CRP were associated with a higher risk of recurrence [80]. Given the role of inflammation in tumour growth and metastasis, inhibiting inflammation could be a potential target for delaying tumour progression.

\section{THE INFLAMMATORY POTENTIAL OF THE DIET AND COLORECTAL CANCER OUTCOMES}

Diet has a marked effect on inflammation [81-83]. Consequently, decreasing the inflammatory potential of the diet could possibly lower inflammation and subsequently delay tumour progression. The complex relationship between nutrition, inflammation and cancer progression is reviewed by Zitvogel and colleagues [84]. In short, an imbalance in food intake, as observed in a western style diet, results in metabolic alterations (metabolic syndrome) and/or obesity. This is linked to chronic subclinical inflammation, which could enhance tumour initiation and progression [84]. Diets high in red and processed meat, and fast foods are associated with higher levels of systemic inflammation, expressed by amongst others higher IL6, TNF $\alpha$, and CRP concentrations, while diets high in fruit, vegetables, and unsaturated fatty acids are associated with lower levels of inflammation in the general population $[82,83]$. The awareness that nutrition influences inflammation has led to the development of dietary inflammatory indexes, of which some are based on nutrients $[64,85]$, while others are based on food groups [86, 87]. A higher risk of allcause mortality was observed with a more pro-inflammatory diet, assessed with indices based on nutrients, at or shortly after diagnosis in CRC patients [88-91]. It is unknown whether the inflammatory potential of the diet also influences cancer recurrence. Given the role of diet in inflammation together with the role of inflammation in tumour progression, decreasing the inflammatory potential of the diet could be a way to hamper CRC progression. 


\section{OBJECTIVES}

The main overarching objective of this thesis is to investigate associations between vitamin $\mathrm{D}$ concentrations, inflammation and their interplay, in relation to CRC outcomes (Figure 3). To address the overall objective several more specific aims are:

1. To investigate which factors determine vitamin D concentrations in CRC patients.

2. To investigate the associations between vitamin $D$ concentrations, magnesium and calcium intake and their interactions in relation to CRC outcomes.

3. To investigate changes in vitamin D concentrations over time in relation to CRC outcomes.

4. To investigate how vitamin D concentrations are associated with levels of inflammation markers over time.

5. To investigate inflammation markers and the inflammatory potential of the diet in relation to $\mathrm{CRC}$ outcomes.

\section{RATIONALE FOR RESEARCH QUESTIONS AND THESIS OUTLINE}

Insights into the role of vitamin D and inflammation in CRC survival could ultimately lead to the development of guidelines regarding vitamin D specified for CRC patients, potentially beneficially affecting CRC prognosis. In this thesis, we investigate the relationship between vitamin $D$, inflammation and their interplay in relation to $C R C$ recurrence and all-cause mortality (Figure 3 ) in two prospective cohort studies in CRC patients in the Netherlands; the COLON study from Wageningen University and the EnCoRe study from Maastricht University. The COLON and EnCoRe studies are described in detail elsewhere $[92,93]$ as well as in the different chapters (2-7) of this thesis. Briefly, CRC patients were recruited upon diagnosis and followed over time. Measurements were done before, during and after treatment, which provides the unique opportunity to investigate changes in vitamin D concentrations and inflammation markers over time in relation to CRC outcomes.

We evaluated which clinical characteristics in conjunction with demographic and lifestyle factors are associated with $25(\mathrm{OH}) \mathrm{D}_{3}$ concentrations at diagnosis and six months later in chapter 2 of this thesis. The association between vitamin D, magnesium and calcium and their interaction in relation to CRC outcomes was investigated in chapter 3 . In chapter 4 of this thesis the association between trajectories of vitamin D concentrations over time and CRC outcomes was examined. The association between vitamin D concentrations and concentrations of inflammatory markers was explored in chapter $\mathbf{5}$. In chapter $\mathbf{6}$ the 
association between levels of inflammation markers and CRC outcomes was investigated. Chapter 7 describes the association between the inflammatory potential of the diet and CRC outcomes. Finally, in chapter $\mathbf{8}$ the main findings of the different chapters are summarized and discussed. In addition, clinical implication are mentioned and recommendations for future research are proposed.

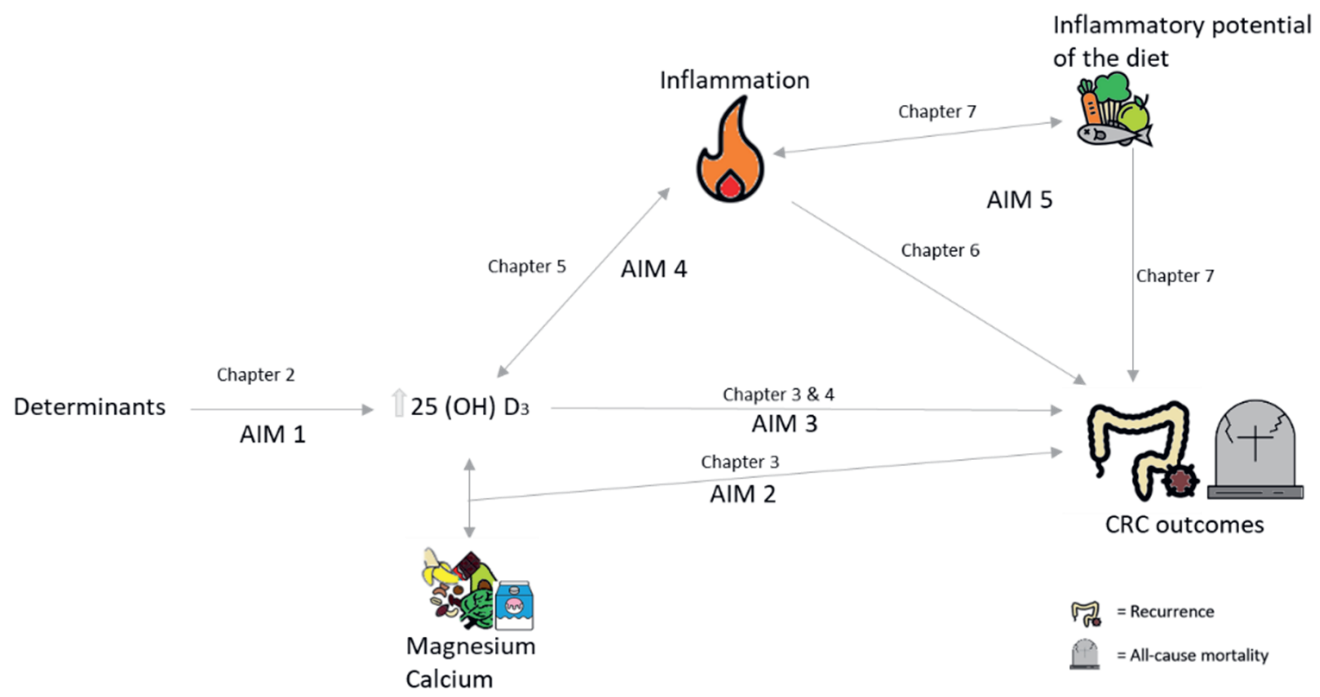

Figure 3: Schematic overview of the study aims that are investigated in this thesis. 


\section{REFERENCES}

[1] Bray F, Ferlay J, Soerjomataram I, Siegel RL, Torre LA, Jemal A. Global cancer statistics 2018: GLOBOCAN estimates of incidence and mortality worldwide for 36 cancers in 185 countries. CA: a cancer journal for clinicians. 2018;68:394-424.

[2] Brouwer NP, Bos AC, Lemmens VE, Tanis PJ, Hugen N, Nagtegaal ID, et al. An overview of 25 years of incidence, treatment and outcome of colorectal cancer patients. International journal of cancer. 2018;143:275866.

[3] Lawrence DP, Kupelnick B, Miller K, Devine D, Lau J. Evidence report on the occurrence, assessment, and treatment of fatigue in cancer patients. Jnci Monographs. 2004;2004:40-50.

[4] Wesselink E, Winkels RM, van Baar H, Geijsen A, van Zutphen M, van Halteren HK, et al. Dietary Intake of Magnesium or Calcium and Chemotherapy-Induced Peripheral Neuropathy in Colorectal Cancer Patients. Nutrients. 2018;10.

[5] Agmon Nardi I, lakobishvili Z. Cardiovascular Risk in Cancer Survivors. Current Treatment Options in Cardiovascular Medicine. 2018;20:47.

[6] Custers JA, Gielissen MF, Janssen SH, de Wilt JH, Prins JB. Fear of cancer recurrence in colorectal cancer survivors. Supportive Care in Cancer. 2016;24:555-62.

[7] Elferink MAG, de Jong KP, Klaase JM, Siemerink EJ, de Wilt JHW. Metachronous metastases from colorectal cancer: a population-based study in North-East Netherlands. International journal of colorectal disease. 2015;30:205-12.

[8] McCullough ML, Zoltick ES, Weinstein SJ, Fedirko V, Wang M, Cook NR, et al. Circulating Vitamin D and Colorectal Cancer Risk: An International Pooling Project of 17 Cohorts. JNCl: Journal of the National Cancer Institute. 2018;111:158-69.

[9] Keum N, Lee DH, Greenwood DC, Manson JE, Giovannucci E. Vitamin D supplementation and total cancer incidence and mortality: a meta-analysis of randomized controlled trials. Annals of Oncology. 2019;30:733-43. [10] Wu G, Xue M, Zhao Y, Han Y, Zhang S, Zhang J, et al. Low circulating 25-hydroxyvitamin D level is associated with increased colorectal cancer mortality: a systematic review and dose-response meta-analysis. Bioscience Reports. 2020;40.

[11] Krishnan AV, Feldman D. Mechanisms of the anti-cancer and anti-inflammatory actions of vitamin D. Annual review of pharmacology and toxicology. 2011;51:311-36.

[12] Mezawa H, Sugiura T, Watanabe M, Norizoe C, Takahashi D, Shimojima A, et al. Serum vitamin D levels and survival of patients with colorectal cancer: post-hoc analysis of a prospective cohort study. BMC Cancer. 2010;10:347.

[13] Fedirko V, Riboli E, Tjønneland A, Ferrari P, Olsen A, Bueno-de-Mesquita HB, et al. Prediagnostic 25hydroxyvitamin D, VDR and CASR polymorphisms, and survival in patients with colorectal cancer in western European populations. Cancer Epidemiology and Prevention Biomarkers. 2012;21:582-93.

[14] Zgaga L, Theodoratou E, Farrington SM, Din FV, Ooi LY, Glodzik D, et al. Plasma Vitamin D Concentration Influences Survival Outcome After a Diagnosis of Colorectal Cancer. Journal of Clinical Oncology. 2014;32:2430-9.

[15] Maalmi H, Walter V, Jansen L, Chang-Claude J, Owen RW, Ulrich A, et al. Relationship of very low serum 25hydroxyvitamin D3 levels with long-term survival in a large cohort of colorectal cancer patients from Germany. European journal of epidemiology. 2017;32:961-71.

[16] Wesa KM, Segal NH, Cronin AM, Sjoberg DD, Jacobs GN, Coleton MI, et al. Serum 25-hydroxy vitamin D and survival in advanced colorectal cancer: a retrospective analysis. Nutrition and cancer. 2015;67:424-30.

[17] Bikle DD. Vitamin D metabolism, mechanism of action, and clinical applications. Chemistry \& biology. 2014;21:319-29.

[18] Seamans KM, Cashman KD. Existing and potentially novel functional markers of vitamin D status: a systematic review. The American journal of clinical nutrition. 2009:ajcn. 27230D.

[19] Cashman KD, Dowling KG, Škrabáková Z, Gonzalez-Gross M, Valtueña J, De Henauw S, et al. Vitamin D deficiency in Europe: pandemic? The American journal of clinical nutrition. 2016;103:1033-44. 
[20] Turner AG, Anderson PH, Morris HA. Vitamin D and bone health. Scandinavian Journal of Clinical and Laboratory Investigation. 2012;72:65-72.

[21] Anglin RE, Samaan Z, Walter SD, McDonald SD. Vitamin D deficiency and depression in adults: systematic review and meta-analysis. The British journal of psychiatry. 2013;202:100-7.

[22] Nowak A, Boesch L, Andres E, Battegay E, Hornemann T, Schmid C, et al. Effect of vitamin D3 on selfperceived fatigue: A double-blind randomized placebo-controlled trial. Medicine. 2016;95.

[23] Reuss-Borst M, Hartmann U, Scheede C, Weiss J. Prevalence of osteoporosis among cancer patients in Germany. Osteoporosis international. 2012;23:1437-44.

[24] Arndt V, Merx H, Stegmaier C, Ziegler H, Brenner H. Restrictions in quality of life in colorectal cancer patients over three years after diagnosis: a population based study. European journal of cancer. 2006;42:1848-57.

[25] Stone P, Richardson A, Ream E, Smith A, Kerr D, Kearney N, et al. Cancer-related fatigue: inevitable, unimportant and untreatable? Results of a multi-centre patient survey. Annals of Oncology. 2000;11:971-5.

[26] Jansen L, Koch L, Brenner H, Arndt V. Quality of life among long-term ( $\geqslant 5$ years) colorectal cancer survivorsSystematic review. European journal of cancer. 2010;46:2879-88.

[27] Lips P, Schoor NM, Jongh RT. Diet, sun, and lifestyle as determinants of vitamin D status. Annals of the New York Academy of Sciences. 2014;1317:92-8.

[28] Holick MF. Vitamin D deficiency. New England Journal of Medicine. 2007;357:266-81.

[29] Touvier M, Deschasaux M, Montourcy M, Sutton A, Charnaux N, Kesse-Guyot E, et al. Determinants of vitamin $D$ status in Caucasian adults: influence of sun exposure, dietary intake, sociodemographic, lifestyle, anthropometric, and genetic factors. Journal of Investigative Dermatology. 2015;135:378-88.

[30] Van Dam RM, Snijder MB, Dekker JM, Stehouwer CD, Bouter LM, Heine RJ, et al. Potentially modifiable determinants of vitamin $D$ status in an older population in the Netherlands: the Hoorn Study-. The American journal of clinical nutrition. 2007;85:755-61.

[31] Brock K, Huang W-Y, Fraser D, Ke L, Tseng M, Stolzenberg-Solomon R, et al. Low vitamin D status is associated with physical inactivity, obesity and low vitamin $D$ intake in a large US sample of healthy middle-aged men and women. The Journal of steroid biochemistry and molecular biology. 2010;121:462-6.

[32] Larose TL, Chen Y, Camargo CA, Langhammer A, Romundstad P, Mai X-M. Factors associated with vitamin D deficiency in a Norwegian population: the HUNT Study. Journal of epidemiology and community health.

2014;68:165-70.

[33] Ng K, Meyerhardt JA, Wu K, Feskanich D, Hollis BW, Giovannucci EL, et al. Circulating 25-hydroxyvitamin d levels and survival in patients with colorectal cancer. Journal of Clinical Oncology. 2008;26:2984-91.

[34] Tretli S, Schwartz GG, Torjesen PA, Robsahm TE. Serum levels of 25-hydroxyvitamin D and survival in Norwegian patients with cancer of breast, colon, lung, and lymphoma: a population-based study. Cancer Causes Control. 2012;23:363-70.

[35] Ng K, Sargent DJ, Goldberg RM, Meyerhardt JA, Green EM, Pitot HC, et al. Vitamin D status in patients with stage IV colorectal cancer: findings from Intergroup trial N9741. Journal of Clinical Oncology. 2011;29:1599-606. [36] Ng K, Venook AP, Sato K, Yuan C, Hollis BW, Niedzwiecki D, et al. Vitamin D status and survival of metastatic colorectal cancer patients: Results from CALGB/SWOG 80405 (Alliance). American Society of Clinical Oncology; 2015.

[37] Freedman DM, Looker AC, Abnet CC, Linet MS, Graubard BI. Serum 25-hydroxyvitamin D and cancer mortality in the NHANES III study (1988-2006). Cancer Res. 2010;70:8587-97.

[38] Vaughan-Shaw PG, Zgaga L, Ooi LY, Theodoratou E, Timofeeva M, Svinti V, et al. Low plasma vitamin D is associated with adverse colorectal cancer survival after surgical resection, independent of systemic inflammatory response. Gut. 2020;69:103-11.

[39] Fakih MG, Trump DL, Johnson CS, Tian L, Muindi J, Sunga AY. Chemotherapy is linked to severe vitamin D deficiency in patients with colorectal cancer. International journal of colorectal disease. 2009;24:219.

[40] Isenring EA, Teleni L, Woodman RJ, Kimlin MG, Walpole E, Karapetis CS, et al. Serum vitamin D decreases during chemotherapy: an Australian prospective cohort study. Asia Pacific journal of clinical nutrition.

2018;27:962. 
[41] Rosanoff A, Dai Q, Shapses SA. Essential nutrient interactions: does low or suboptimal magnesium status interact with vitamin D and/or calcium status? Advances in Nutrition. 2016;7:25-43.

[42] Lips P. Interaction between vitamin D and calcium. Scandinavian Journal of Clinical and Laboratory Investigation. 2012;72:60-4.

[43] Uwitonze AM, Razzaque MS. Role of magnesium in vitamin D activation and function. The Journal of the American Osteopathic Association 2018;118:181-9.

[44] Deng X, Song Y, Manson JE, Signorello LB, Zhang SM, Shrubsole MJ, et al. Magnesium, vitamin D status and mortality: results from US National Health and Nutrition Examination Survey (NHANES) 2001 to 2006 and NHANES III. BMC medicine. 2013;11:187.

[45] Yang B, McCullough ML, Gapstur SM, Jacobs EJ, Bostick RM, Fedirko V, et al. Calcium, vitamin D, dairy products, and mortality among colorectal cancer survivors: the Cancer Prevention Study-II Nutrition Cohort. Journal of Clinical Oncology. 2014;32:2335-43.

[46] Zhu Y, Wang PP, Zhai G, Bapat B, Savas S, Woodrow JR, et al. Vitamin D receptor and calcium-sensing receptor polymorphisms and colorectal cancer survival in the Newfoundland population. British journal of cancer. 2017;117:898-906.

[47] Fedirko V, Riboli E, Tjonneland A, Ferrari P, Olsen A, Bueno-de-Mesquita HB, et al. Prediagnostic 25hydroxyvitamin $D, V D R$ and CASR polymorphisms, and survival in patients with colorectal cancer in western European ppulations. Cancer epidemiology, biomarkers \& prevention : a publication of the American Association for Cancer Research, cosponsored by the American Society of Preventive Oncology. 2012;21:582-93.

[48] Facciorusso A, Del Prete V, Muscatiello N, Crucinio N, Barone M. Prognostic role of 25-hydroxyvitamin D in patients with liver metastases from colorectal cancer treated with radiofrequency ablation. Journal of gastroenterology and hepatology. 2016;31:1483-8.

[49] Yang L, Chen H, Zhao M, Peng P. Prognostic value of circulating vitamin D binding protein, total, free and bioavailable 25-hydroxy vitamin D in patients with colorectal cancer. Oncotarget. 2017;8:40214-21.

[50] Cooney RV, Chai W, Franke AA, Wilkens LR, Kolonel LN, Le Marchand L. C-reactive protein, lipid-soluble micronutrients, and survival in colorectal cancer patients. Cancer Epidemiology, Biomarkers \& Prevention. 2013;22:1278-88.

[51] Vaughan-Shaw PG, Buijs LF, Blackmur JP, Theodoratou E, Zgaga L, Din FVN, et al. The effect of vitamin D supplementation on survival in patients with colorectal cancer: systematic review and meta-analysis of randomised controlled trials. British Journal of Cancer. 2020.

[52] Feldman D, Krishnan AV, Swami S, Giovannucci E, Feldman BJ. The role of vitamin D in reducing cancer risk and progression. Nature reviews Cancer. 2014;14:342-57.

[53] Krishnan AV, Feldman D. Mechanisms of the anti-cancer and anti-inflammatory actions of vitamin D. Annual review of pharmacology and toxicology. 2011;51:311-36.

[54] Leyssens C, Verlinden L, Verstuyf A. Antineoplastic effects of 1,25(OH)2D3 and its analogs in breast, prostate and colorectal cancer. Endocrine-related cancer. 2013;20:R31-47.

[55] Skrajnowska D, Bobrowska-Korczak B. Potential molecular mechanisms of the anti-cancer activity of vitamin D. Anticancer research. 2019;39:3353-63.

[56] Liu W, Zhang L, Xu H-J, Li Y, Hu C-M, Yang J-Y, et al. The anti-inflammatory effects of vitamin D in tumorigenesis. International journal of molecular sciences. 2018;19:2736.

[57] van Harten-Gerritsen AS, Balvers MG, Witkamp RF, Kampman E, van Duijnhoven FJ. Vitamin D, inflammation and colorectal cancer progression: A review of mechanistic studies and future directions for epidemiological studies. Cancer Epidemiology and Prevention Biomarkers. 2015:cebp. 0601.2015.

[58] Cohen-Lahav M, Shany S, Tobvin D, Chaimovitz C, Douvdevani A. Vitamin D decreases NFkappaB activity by increasing IkappaBalpha levels. Nephrology, dialysis, transplantation : official publication of the European Dialysis and Transplant Association - European Renal Association. 2006;21:889-97.

[59] Jeon SM, Shin EA. Exploring vitamin D metabolism and function in cancer. Exp Mol Med. 2018;50:20.

[60] Liu T, Zhang L, Joo D, Sun S-C. NF-KB signaling in inflammation. Signal Transduction and Targeted Therapy.

2017;2:17023. 
[61] West NR, McCuaig S, Franchini F, Powrie F. Emerging cytokine networks in colorectal cancer. Nature Reviews Immunology. 2015;15:615-29.

[62] Aoki T, Narumiya S. Prostaglandins and chronic inflammation. Trends in pharmacological sciences.

2012;33:304-11.

[63] Hopkins MH, Owen J, Ahearn T, Fedirko V, Flanders WD, Jones DP, et al. Effects of supplemental vitamin D and calcium on biomarkers of inflammation in colorectal adenoma patients: a randomized, controlled clinical trial. Cancer prevention research. 2011;4:1645-54.

[64] van Woudenbergh GJ, Theofylaktopoulou D, Kuijsten A, Ferreira I, van Greevenbroek MM, van der Kallen CJ, et al. Adapted dietary inflammatory index and its association with a summary score for low-grade inflammation and markers of glucose metabolism: the Cohort study on Diabetes and Atherosclerosis Maastricht (CODAM) and the Hoorn study. The American journal of clinical nutrition. 2013;98:1533-42.

[65] Greten FR, Grivennikov SI. Inflammation and Cancer: Triggers, Mechanisms, and Consequences. Immunity. 2019;51:27-41.

[66] Chang PH, Pan YP, Fan CW, Tseng WK, Huang JS, Wu TH, et al. Pretreatment serum interleukin-1 $\beta$, interleukin-6, and tumor necrosis factor- $\alpha$ levels predict the progression of colorectal cancer. Cancer medicine. 2016;5:426-33.

[67] Seruga B, Zhang H, Bernstein LJ, Tannock IF. Cytokines and their relationship to the symptoms and outcome of cancer. Nature Reviews Cancer. 2008;8:887.

[68] Xu J, Ye Y, Zhang H, Szmitkowski M, Mäkinen M, Li P, et al. Diagnostic and prognostic value of serum interleukin-6 in colorectal cancer. Medicine. 2016;95.

[69] Knupfer H, Preiss R. Serum interleukin-6 levels in colorectal cancer patients--a summary of published results. International journal of colorectal disease. 2010;25:135-40.

[70] Abtahi S, Davani F, Mojtahedi Z, Hosseini SV, Bananzadeh A, Ghaderi A. Dual association of serum interleukin-10 levels with colorectal cancer. Journal of cancer research and therapeutics. 2017;13:252.

[71] Stanilov N, Miteva L, Dobreva Z, Stanilova S. Colorectal cancer severity and survival in correlation with tumour necrosis factor-alpha. Biotechnology \& Biotechnological Equipment. 2014;28:911-7.

[72] Xia W, Chen W, Zhang Z, Wu D, Wu P, Chen Z, et al. Prognostic value, clinicopathologic features and diagnostic accuracy of interleukin-8 in colorectal cancer: a meta-analysis. PloS one. 2015;10:e0123484.

[73] Grivennikov SI, Greten FR, Karin M. Immunity, inflammation, and cancer. Cell. 2010;140:883-99.

[74] Qian B-Z, Pollard JW. Macrophage diversity enhances tumor progression and metastasis. Cell. 2010;141:3951.

[75] Sproston NR, Ashworth JJ. Role of C-Reactive Protein at Sites of Inflammation and Infection. Frontiers in immunology. 2018;9:754.

[76] Li J, Huang L, Zhao H, Yan Y, Lu J. The Role of Interleukins in Colorectal Cancer. International Journal of Biological Sciences. 2020;16:2323-39.

[77] Rasic I, Radovic S, Aksamija G. Relationship between chronic inflammation and the stage and histopathological size of colorectal carcinoma. Medical Archives. 2016;70:104.

[78] Szkaradkiewicz A, Marciniak R, Chudzicka-Strugała I, Wasilewska A, Drews M, Majewski P, et al. Proinflammatory cytokines and IL-10 in inflammatory bowel disease and colorectal cancer patients. Archivum immunologiae et therapiae experimentalis. 2009;57:291.

[79] Bălăşoiu M, Bălăşoiu AT, Mogoantă SŞ, Bărbălan A, Stepan AE, Ciurea RN, et al. Serum and tumor microenvironment IL-8 values in different stages of colorectal cancer. Rom J Morphol Embryol. 2014;55:575-8.

[80] Matsubara D, Arita T, Nakanishi M, Kuriu Y, Murayama Y, Kudou M, et al. The impact of postoperative inflammation on recurrence in patients with colorectal cancer. International Journal of Clinical Oncology. 2020;25:602-13.

[81] Wiseman MJ. Nutrition and cancer: prevention and survival. British Journal of Nutrition. 2018:1-7.

[82] Galland L. Diet and inflammation. Nutrition in Clinical Practice. 2010;25:634-40.

[83] Barbaresko J, Koch M, Schulze MB, Nöthlings U. Dietary pattern analysis and biomarkers of low-grade inflammation: a systematic literature review. Nutrition reviews. 2013;71:511-27.

[84] Zitvogel L, Pietrocola F, Kroemer G. Nutrition, inflammation and cancer. Nat Immunol. 2017;18:843-50. 
[85] Shivappa N, Steck SE, Hurley TG, Hussey JR, Hebert JR. Designing and developing a literature-derived, population-based dietary inflammatory index. Public health nutrition. 2014;17:1689-96.

[86] Tabung FK, Smith-Warner SA, Chavarro JE, Wu K, Fuchs CS, Hu FB, et al. Development and Validation of an Empirical Dietary Inflammatory Index. The Journal of nutrition. 2016;146:1560-70.

[87] Joanna K, Holly H, Håkan M, Karl M, Alicja W. Questionnaire-Based Anti-Inflammatory Diet Index as a Predictor of Low-Grade Systemic Inflammation. Antioxidants \& Redox Signaling. 2018;28:78-84.

[88] Deng FE, Shivappa N, Tang Y, Mann JR, Hebert JR. Association between diet-related inflammation, all-cause, all-cancer, and cardiovascular disease mortality, with special focus on prediabetics: findings from NHANES III. European journal of nutrition. 2017;56:1085-93.

[89] Ratjen I, Shivappa N, Schafmayer C, Burmeister G, Nothlings U, Hampe J, et al. Association between the dietary inflammatory index and all-cause mortality in colorectal cancer long-term survivors. International journal of cancer Journal international du cancer. 2019;144:1292-301.

[90] Zheng J, Tabung FK, Zhang J, Murphy EA, Shivappa N, Ockene JK, et al. Post-cancer diagnosis dietary inflammatory potential is associated with survival among women diagnosed with colorectal cancer in the Women's Health Initiative. European journal of nutrition. 2019.

[91] Galas A, Kulig J. Low-grade dietary-related inflammation and survival after colorectal cancer surgery. Journal of cancer research and clinical oncology. 2014;140:1517-25.

[92] Winkels RM, Heine-Broring RC, van Zutphen M, van Harten-Gerritsen S, Kok DE, van Duijnhoven FJ, et al. The COLON study: Colorectal cancer: Longitudinal, Observational study on Nutritional and lifestyle factors that may influence colorectal tumour recurrence, survival and quality of life. BMC Cancer. 2014;14:374.

[93] van Roekel EH, Bours MJ, de Brouwer CP, Ten Napel H, Sanduleanu S, Beets GL, et al. The Applicability of the International Classification of Functioning, Disability, and Health to Study Lifestyle and Quality of Life of Colorectal Cancer Survivors. Cancer epidemiology, biomarkers \& prevention : a publication of the American Association for Cancer Research, cosponsored by the American Society of Preventive Oncology. 2014;23:1394405. 



\title{
CHAPTER 2
}

\section{CHEMOTHERAPY AND VITAMIN D SUPPLEMENT USE ARE DETERMINANTS OF CIRCULATING VITAMIN D LEVELS DURING THE FIRST SIX MONTHS AFTER COLORECTAL CANCER DIAGNOSIS.}

\author{
Evertine Wesselink \\ Martijn J.L. Bours \\ Johannes H.W. de Wilt \\ Michiel Aquarius \\ Stephanie O. Breukink \\ Bibi Hansson \\ Eric T.P. Keulen \\ Dieuwertje E. Kok \\ Jody van den Ouweland \\ Eline H. van Roekel \\ Merel Snellen \\ Renate Winkels \\ Renger F. Witkamp \\ Moniek van Zutphen \\ Matty P. Weijenberg \\ Ellen Kampman \\ Franzel J.B. van Duijnhoven
}

The Journal of steriod biochemistry and molecular biology 2020 May;199:105577 


\section{ABSTRACT}

Background: Vitamin D metabolites, including 25-hydroxyvitamin D3 $\left(25(\mathrm{OH}) \mathrm{D}_{3}\right)$, may inhibit colorectal cancer (CRC) progression. Here we investigated cross-sectional and longitudinal associations of demographic, lifestyle and clinical characteristics with serum $25(\mathrm{OH}) \mathrm{D}_{3}$ concentrations in CRC patients at diagnosis and six months later.

Methods: In 1,201 newly-diagnosed stage I-III CRC patients, $25(\mathrm{OH}) \mathrm{D}_{3}$ levels were analysed twice. Multivariable linear regression was used to assess demographic, lifestyle and clinical determinants of $25(\mathrm{OH}) \mathrm{D}_{3}$ levels at diagnosis and six months later. Linear mixed models were used to assess characteristics associated with changes in $25(\mathrm{OH}) \mathrm{D}_{3}$ levels over time.

Results: Vitamin D intake from diet or supplements, use of calcium supplements, BMI and disease stage were associated with $25(\mathrm{OH}) \mathrm{D}_{3}$ levels at both time points. Six months after diagnosis, gender and having received chemo- and/or radiotherapy were also associated with $25(\mathrm{OH}) \mathrm{D}_{3}$ levels. A stronger decrease in $25(\mathrm{OH}) \mathrm{D}_{3}$ levels was observed in patients who underwent chemotherapy, compared to surgery only $(\beta-6.9 \mathrm{nmol} / \mathrm{L} 95 \% \mathrm{Cl}-9.8 ;-4.0)$. Levels of $25(\mathrm{OH}) \mathrm{D}_{3}$ levels increased in patients using vitamin $\mathrm{D}$ supplements compared to non-users ( $\beta 4.0 \mathrm{nmol} / \mathrm{L} \mathrm{95 \% Cl} \mathrm{1.2;6.8).}$

Conclusion: Vitamin D supplement use and cancer treatment appear to be important determinants of $25(\mathrm{OH}) \mathrm{D}_{3}$ levels during the first six months after $\mathrm{CRC}$ diagnosis, although the difference in $25(\mathrm{OH}) \mathrm{D}_{3}$ levels was minor.

KEYWORDS: serum $25(\mathrm{OH}) \mathrm{D}_{3}$, vitamin $\mathrm{D}$, colorectal cancer, patients, changes over time, lifestyle and clinical determinants, 


\section{INTRODUCTION}

The incidence of colorectal cancer (CRC) and the number of cancer survivors is increasing [1]. Therefore, it is important to optimize quality of life and long term survival in CRC patients. Vitamin $D$ is a potentially interesting compound as most studies on the association between 25-hydroxyvitamin $\mathrm{D} 3\left(25(\mathrm{OH}) \mathrm{D}_{3}\right)$ blood levels at diagnosis of CRC and disease outcomes point at higher survival rates with higher levels of $25(\mathrm{OH}) \mathrm{D}_{3}$ [2]. Five out of the seven published studies reported hazard ratios ranging between 0.52 and 0.70 for all-cause mortality [3-7], while only two did not find a statistically significant association [8,9]. In addition, daily vitamin D supplementation was associated with a reduced total cancer mortality risk in the general population [10]. Besides, low $25(\mathrm{OH}) \mathrm{D}_{3}$ levels are associated with lower bone density, depression and fatigue in the general population [11-13] and likely also in CRC patients. Indeed, the prevalence of osteoporosis is higher among cancer patients compared to the general community dwelling population [14]. Furthermore, fatigue and depression are often reported in CRC patients even years after diagnosis and are seriously affecting their quality of life [15-17]. Vitamin D deficiency $\left(25(\mathrm{OH}) \mathrm{D}_{3}\right.$ levels $\left.<50 \mathrm{nmol} / \mathrm{L}\right)[18]$ at diagnosis were reported to occur in $40-87 \%$ of CRC patients $[4-7,19]$, while vitamin $D$ deficiency was reported in $29-58 \%$ of healthy individuals of comparable age [20-23]. This high prevalence of vitamin D deficiency in CRC patients, together with the possible beneficial effects of $25(\mathrm{OH}) \mathrm{D}_{3}$ on quality of life, cancer progression and survival warrants further study on determinants of serum $25(\mathrm{OH}) \mathrm{D}_{3}$ levels to eventually optimize $25(\mathrm{OH}) \mathrm{D}_{3}$ levels in CRC patients.

Several factors may influence $25(\mathrm{OH}) \mathrm{D}_{3}$ blood concentrations in CRC patients. Levels may be influenced by the disease itself and (or) cancer treatment [24-26]. Vitamin D deficiency was found to be more prevalent in rectal cancer patients compared to colon cancer patients [26]. Furthermore, low $25(\mathrm{OH}) \mathrm{D}_{3}$ levels were found in CRC patient cohorts in which $25(\mathrm{OH}) \mathrm{D}_{3}$ levels were measured shortly after surgery with a median of $25 \mathrm{nmol} / \mathrm{L}$ $[4,6,7]$, while other studies measuring $25(\mathrm{OH}) \mathrm{D}_{3}$ levels before or at diagnosis, thus before surgery, found $25(\mathrm{OH}) \mathrm{D}_{3}$ levels ranging between 43 and $65 \mathrm{nmol} / \mathrm{L}[3,5,8,9,19,27,28]$. In addition, Fakih and colleagues observed that chemotherapy was associated with vitamin $D$ deficiency in CRC patients [26]. To our knowledge, only one small study investigated serum $25(\mathrm{OH}) \mathrm{D}_{3}$ levels prospectively and observed that chemotherapy was associated with a decreased 25(OH)D level in 85 cancer patients [29].

In addition, $25(\mathrm{OH}) \mathrm{D}_{3}$ levels are likely to be modulated by diet and lifestyle. In the general population several lifestyle factors are known to be associated with $25(\mathrm{OH}) \mathrm{D}_{3}$ levels [2023]. Higher physical activity levels, the use of vitamin $D$ supplements and dietary vitamin $D$ $[20-23,30]$, and calcium intake $[22,30]$ were associated with higher $25(\mathrm{OH}) \mathrm{D}_{3}$ levels, while obesity was associated with lower $25(\mathrm{OH}) \mathrm{D}_{3}$ levels $[20-23,30]$. However, the relative importance of these dietary and lifestyle factors in conjunction with clinical factors in CRC 
patients is presently unknown. In addition, $25(\mathrm{OH}) \mathrm{D}_{3}$ levels may also change over time in CRC patients, because of changes in diet and/or lifestyle due to the diagnosis or treatment [31].

The aim of the current study was to investigate which clinical characteristics in conjunction with demographic and lifestyle factors, were associated with $25(\mathrm{OH}) \mathrm{D}_{3}$ levels at diagnosis and six months later. Besides, we determined which characteristics were associated with changes in $25(\mathrm{OH}) \mathrm{D}_{3}$ levels in the first six months after diagnosis in $\mathrm{CRC}$ patients.

\section{METHODS}

\section{STUDY POPULATION}

In total, 1733 CRC patients were recruited in two ongoing prospective cohort studies in the Netherlands between August 2010 and October 2015. Of these patients, 1,201 donated a blood sample at diagnosis and 1,002 also six months after diagnosis (Figure 1). The design of the COLON study [32] as well as the EnCoRe study [33] has been described earlier. In short, newly diagnosed CRC patients were recruited directly after diagnosis in 14 hospitals and were followed during and after treatment. Men and women of all ages, with stage I-III CRC were eligible for this analysis. Non-Dutch speaking patients, or patients with a history of CRC or (partial) bowel resection, chronic inflammatory bowel disease, hereditary CRC syndromes (e.g. Lynch syndrome, Familial Adenomatous Polyposis, PeutzJegher), dementia or another mental condition obstructing participation were excluded from the study.

In both studies, the first round of data collection was done at diagnosis. In the COLON study the second data collection time point was six months after diagnosis. For the EnCoRe cohort, data were collected at six weeks and at six months after the end of treatment. To be able to pool the data of both cohorts at comparable time points since CRC diagnosis, we selected the time point in the EnCoRe cohort closest to six months (mean 6.7 months) after diagnosis as the second time point for these analyses. 


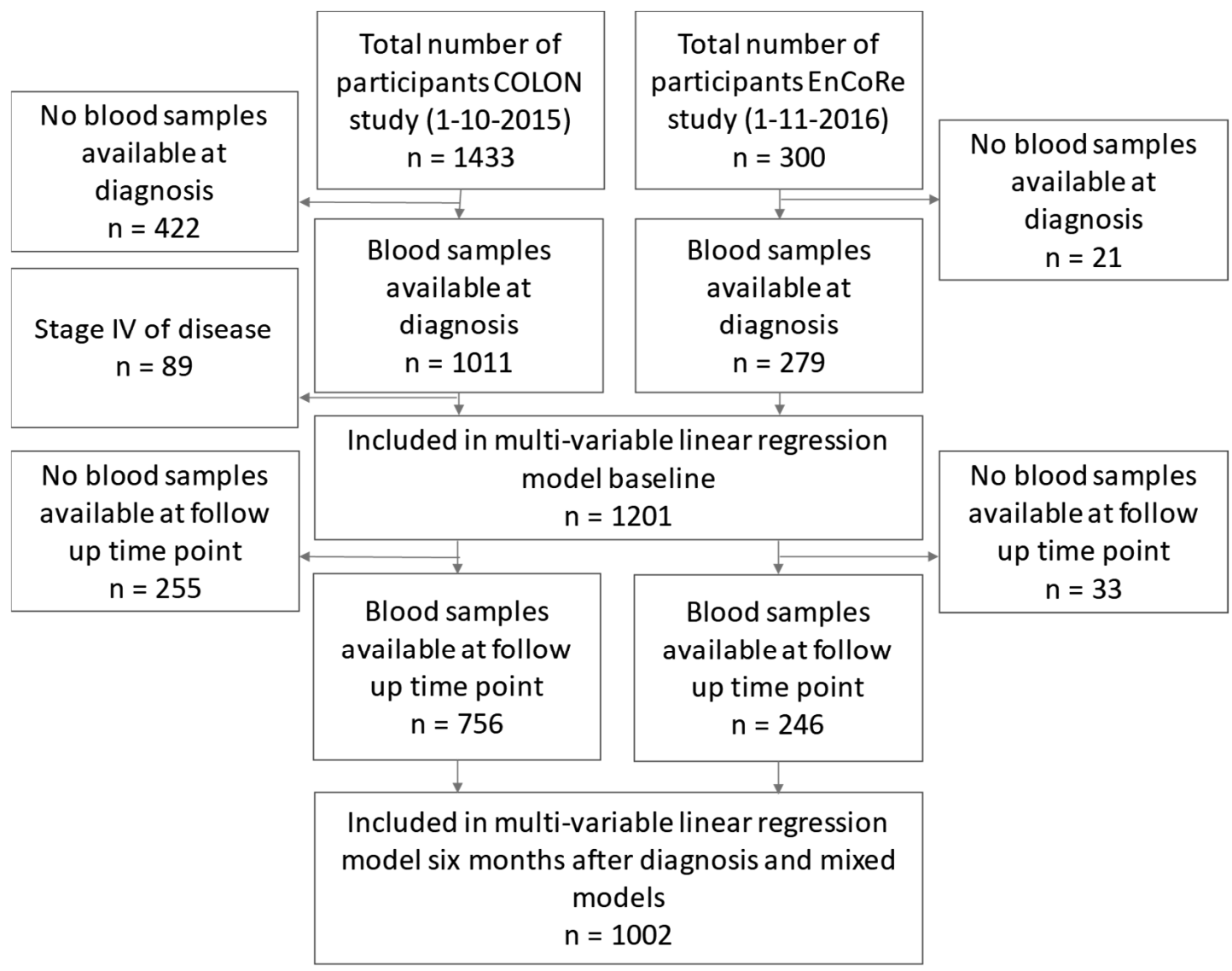

Figure 1: Flowchart representing patient selection for the current study. Colorectal cancer patients participating in the COLON study the EnCoRe study and donating blood at diagnosis were included in the current study. Patients who also donated a blood at the follow up time point were included in analyses regarding determinants of vitamin $D$ status 6 months after diagnosis and the analyses regarding the determinants of changes in vitamin D status over time. 


\section{BLOOD COLLECTION AND VITAMIN D CONCENTRATIONS}

For the COLON study, blood samples were obtained during a regular clinical visit in the hospital at diagnosis and six months after diagnosis. Blood samples at diagnosis were collected before start of treatment in $91 \%$ of the patients included in these analyses. For the EnCoRe study, blood samples at diagnosis were obtained during a regular clinical visit in the hospital or by a research assistant during a home visit before start of treatment. All blood samples at six weeks and six months after the end of treatment were collected by a research assistant during home visit. All blood samples were obtained in a serum tube, centrifuged and aliquoted into serum and were immediately stored in a freezer at $-80^{\circ} \mathrm{C}$ until further analysis.

For both cohorts, serum $25(\mathrm{OH}) \mathrm{D}_{3}$ levels were measured by isotope-dilution liquid chromatography tandem mass spectrometry (LC-MS/MS) in the Canisius Wilhelmina Hospital, Nijmegen, the Netherlands [34]. The inter-assay coefficients of variation were $5.3 \%, 3.1 \%$ and $2.9 \%$ at $25(\mathrm{OH}) \mathrm{D}_{3}$ concentrations of $39.0,92.5$ and $127.0 \mathrm{nmol} / \mathrm{L}$, respectively. Concentrations of 25(OH)D2 were all below the detection limit in our population.

\section{DEMOGRAPHIC AND LIFESTYLE DETERMINANTS}

Information on height, weight, waist and hip circumference was collected using selfadministered questionnaires in the COLON study. In the EnCoRe study, these measurements were performed during home visits. BMI was calculated and categorized as normal weight $\left(\mathrm{BMI}<25 \mathrm{~kg} / \mathrm{m}^{2}\right)$, overweight (BMI $\left.25-29 \mathrm{~kg} / \mathrm{m}^{2}\right)$, or obesity $\left(\mathrm{BMI} \geq 30 \mathrm{~kg} / \mathrm{m}^{2}\right)$. The waist-hip ratio (WHR) was calculated by dividing the waist circumference $(\mathrm{cm})$ by the hip circumference $(\mathrm{cm})$. Low education was defined as primary school and lower general secondary education; medium as lower vocational training and higher general secondary education; high as high vocational training and university.

Physical activity was assessed using the Short QUestionnaire to ASsess Health-enhancing physical activity (SQUASH) in both cohorts [35]. Activities with a Metabolic Equivalent score (MET score) $\geq 4$ were defined as moderate to vigorous physical activity in patients aged between 18 and 55 and MET score $\geq 3$ for patients $>55$ years. Habitual dietary intake in the month (COLON study) or year (EnCoRe study) preceding diagnosis was assessed using a semi-quantitative food frequency questionnaire. Six months after diagnosis habitual dietary intake was assessed with a semi-quantitative food frequency questionnaire in the COLON study and a 7 day food diary in the EnCoRe study. Average daily vitamin $\mathrm{D}(\mu \mathrm{g} /$ day), calcium (mg/day) and alcohol (g/day) intakes were calculated using the 2011 Dutch food composition table [36]. Supplement use was assessed by a dietary supplement questionnaire developed by the Division of Human Nutrition \& Health of Wageningen University and Research [32] in the COLON study. In the EnCoRe study supplement use was assessed by a dietician during a home visit, using standardized forms, and by asking the participants to show the packaging. Supplement use 
was defined as using supplements at least once a week for at least one month.

Additionally, vitamins or minerals that were used once a month, but contained a high dose to cover the intake for a longer period of time (e.g. D-CURE 25.000 IE Cholecalciferol supplementation), were also classified as supplement use.

\section{CLINICAL INFORMATION}

Clinical data, such as stage of disease, tumour location, tumour differentiation, histological type, date of start treatment, type of treatment (surgery, neo-adjuvant/adjuvant chemotherapy, radiation therapy) and presence of comorbidities (yes/no, diabetes, endocrine disorders, cardiovascular, infectious, gastro-intestinal, muscular and joint, neurologic, pulmonary and urogenital diseases) for both cohorts were derived from the Dutch ColoRectal Audit (DCRA) [37].

\section{StATISTiCAl ANALYSES}

To correct for the influence of season on serum vitamin $D$ levels, $25(\mathrm{OH}) \mathrm{D}_{3}$ were adjusted for week of blood collection using the locally weighted polynomial regression (LOESS) method $[38,39]$. To obtain interpretable $25(\mathrm{OH}) \mathrm{D}_{3}$ level values, we added the median $25(\mathrm{OH}) \mathrm{D}_{3}$ level to the residuals of the LOESS method. These adjusted $25(\mathrm{OH}) \mathrm{D}_{3}$ levels were used in all subsequent statistical analyses. Population characteristics were described as percentages and medians with interquartile range [IQR] for the total study population and stratified by vitamin $D$ deficiency ( $<50 \mathrm{nmol} / \mathrm{L}$ ) at diagnosis and six months after diagnosis. Potential determinants of $25(\mathrm{OH}) \mathrm{D}_{3}$ levels were age (continuous, years), gender (men, women), education (low, medium, high), BMI (normal, overweight, obese and continuous), WHR (continuous), smoking (current, former, never), physical activity (hours moderate-to-vigorous physical activity/week), vitamin D supplement use (yes, no), calcium supplement use (yes, no), dietary vitamin D intake ( $\mu \mathrm{g} /$ day, continuous), dietary calcium intake (mg/day, continuous), alcohol intake (g/day, continuous), tumour stage (I, II, III), tumour location (colon, rectum), tumour differentiation (good/moderate, bad), histological type (adenocarcinoma, other), cancer treatment (surgery only, surgery + neoadjuvant/adjuvant chemotherapy, surgery + radiotherapy, surgery + chemo radiation therapy) and any of the assessed comorbidities (yes, no). Lifestyle and demographic determinants measured at diagnosis were used to assess associations with $25(\mathrm{OH}) \mathrm{D}_{3}$ at diagnosis and determinants measured six months after diagnosis to assess the association with $25(\mathrm{OH}) \mathrm{D}_{3}$ levels six months after diagnosis. Clinical determinants, as stage of disease, type of treatment and having comorbidities were assessed once, shortly after diagnosis.

Linear regression analysis was used to assess which determinants were associated with $25(\mathrm{OH}) \mathrm{D}_{3}$ levels at diagnosis and six months after diagnosis. Only when potential determinants by themselves were statistically significantly associated with $25(\mathrm{OH}) \mathrm{D}_{3}$ levels, they were added to the multivariable model. Determinants added based on 
univariate regression analyses were gender, BMI, WHR, smoking, physical activity, stage of disease, alcohol intake and vitamin D supplements. The use of calcium supplements was not added to the multivariable model as this was highly correlated with the use of vitamin D supplements (89\% of patients using calcium containing supplements were also using vitamin D containing supplements). Additionally, age, having comorbidities, dietary calcium intake and vitamin D intake were added to the multivariable model, which was based on associations with $25(\mathrm{OH}) \mathrm{D}_{3}$ levels being reported in literature [20-23,30] for both time points. For the analyses six months after diagnosis, $25(\mathrm{OH}) \mathrm{D}_{3}$ levels at diagnosis and cancer treatment were also added to the models as covariates. Stage was not added to the multivariable model as covariate since type of treatment is dependent on stage of disease and are therefore highly correlated. All models were adjusted for cohort.

Linear mixed models were used to model $25(\mathrm{OH}) \mathrm{D}_{3}$ levels over time to examine changes in $25(\mathrm{OH}) \mathrm{D}_{3}$ levels from diagnosis to six months after diagnosis and to examine which determinants were associated with these changes. Only patients who donated blood at both time points $(n=1002)$ were included in these analyses. Time was added as a binary variable. As fixed effects we included cohort, age, gender, BMI, smoking status, physical activity, type of treatment, vitamin D supplement use, and dietary intake of vitamin $D$, calcium and alcohol. All fixed effects were also included in the model as an interaction term with time. Only significant interactions were retained, which included (time*type of treatment, time*WHR, time*vitamin D supplement use and time*comorbidities).

Sensitivity analyses were performed including only patients whose baseline blood sample was collected before treatment commenced (91\%).

Statistical analyses were performed in SAS 9.4 (SAS Institute, Cary NC). P-values $<0.05$ were considered statistically significant.

\section{RESULTS}

\section{PATIENT CHARACTERISTICS}

A total of 1,201 CRC patients were included in the analyses of whom 779 (65\%) were men. Median age at CRC diagnosis was 67 years [IQR 62-73] and 308 (27\%) had CRC stage I of disease, 341 (29\%) were diagnosed with stage II and 504 (44\%) with stage III. At diagnosis, the median serum $25(\mathrm{OH}) \mathrm{D}_{3}$ level was 53 [IQR 41-67] nmol/L. Six months after diagnosis the median serum $25(\mathrm{OH}) \mathrm{D}_{3}$ level was 48 [37-63] nmol/L. In our study population, $45 \%$ of the patients were vitamin D deficient ( $<50 \mathrm{nmol} / \mathrm{L}$ ) at diagnosis, while this was $54 \%$ six months after diagnosis. Twenty percent of the patients who were vitamin $D$ sufficient at diagnosis were vitamin $D$ deficient six months after diagnosis. Nine percent of the patients were deficient at diagnosis and sufficient six months after diagnosis. Thirty-seven percent of patients were deficient at both time points and $35 \%$ were sufficient at both time points. 
Patients' characteristics stratified by vitamin D deficiency at diagnosis and six months after diagnosis are shown in Table 1. At both time points, patients who had a deficient $25(\mathrm{OH}) \mathrm{D}_{3}$ status appeared to be more often male, obese, had a lower level of physical activity, a more advanced disease stage, were less often using vitamin D and calcium supplements and had a lower alcohol intake compared with patients who had sufficient levels. In addition, six months after diagnosis patients with a deficient $25(\mathrm{OH}) \mathrm{D}_{3}$ status more often received chemotherapy and/or radiotherapy compared with patients with a sufficient vitamin D status.

\section{DeterminantS OF 25(OH)D 3 LEVELS}

At diagnosis, several demographic, lifestyle and clinical factors were statistically significantly associated with $25(\mathrm{OH}) \mathrm{D}_{3}$ levels (Table 2 ). In the multivariable analyses, the use of vitamin D supplements, use of calcium supplements, higher dietary vitamin D intake, higher alcohol intake, and being more physically active were associated with higher $25(\mathrm{OH}) \mathrm{D}_{3}$ levels. A higher BMI and WHR, a high education level compared to a low education level, and stage III compared to stage I of disease were associated with lower $25(\mathrm{OH}) \mathrm{D}_{3}$ levels.

Six months after diagnosis, $25(\mathrm{OH}) \mathrm{D}_{3}$ levels at diagnosis, having comorbidities, the use of vitamin D and calcium supplements and dietary vitamin D intake were statistically significantly associated with higher $25(\mathrm{OH}) \mathrm{D}_{3}$ levels. Male gender, a higher BMI, chemotherapy as well as radiotherapy compared to surgery only, and stage III compared to stage I of disease were associated with lower $25(\mathrm{OH}) \mathrm{D}_{3}$ levels 


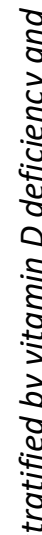

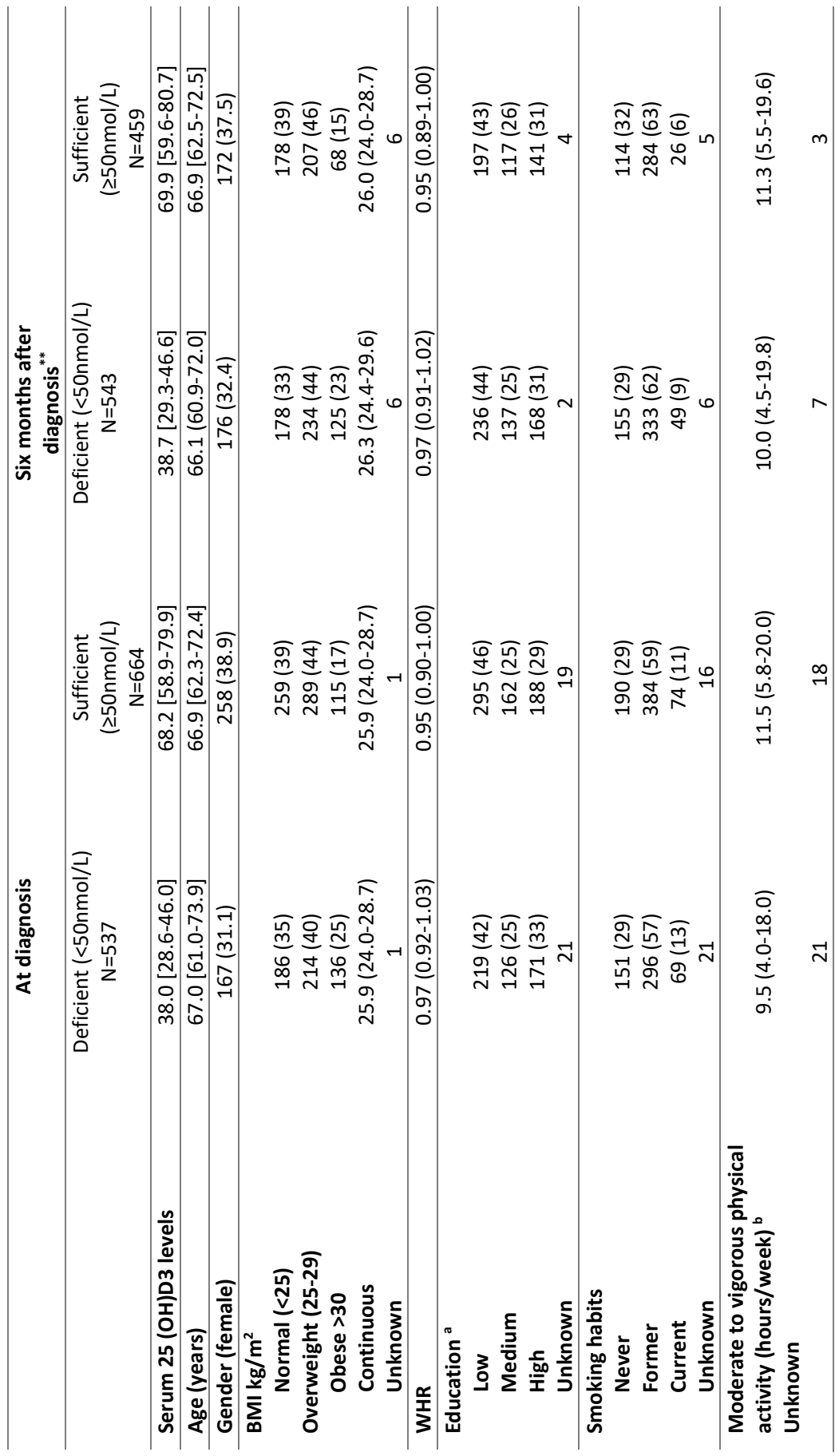




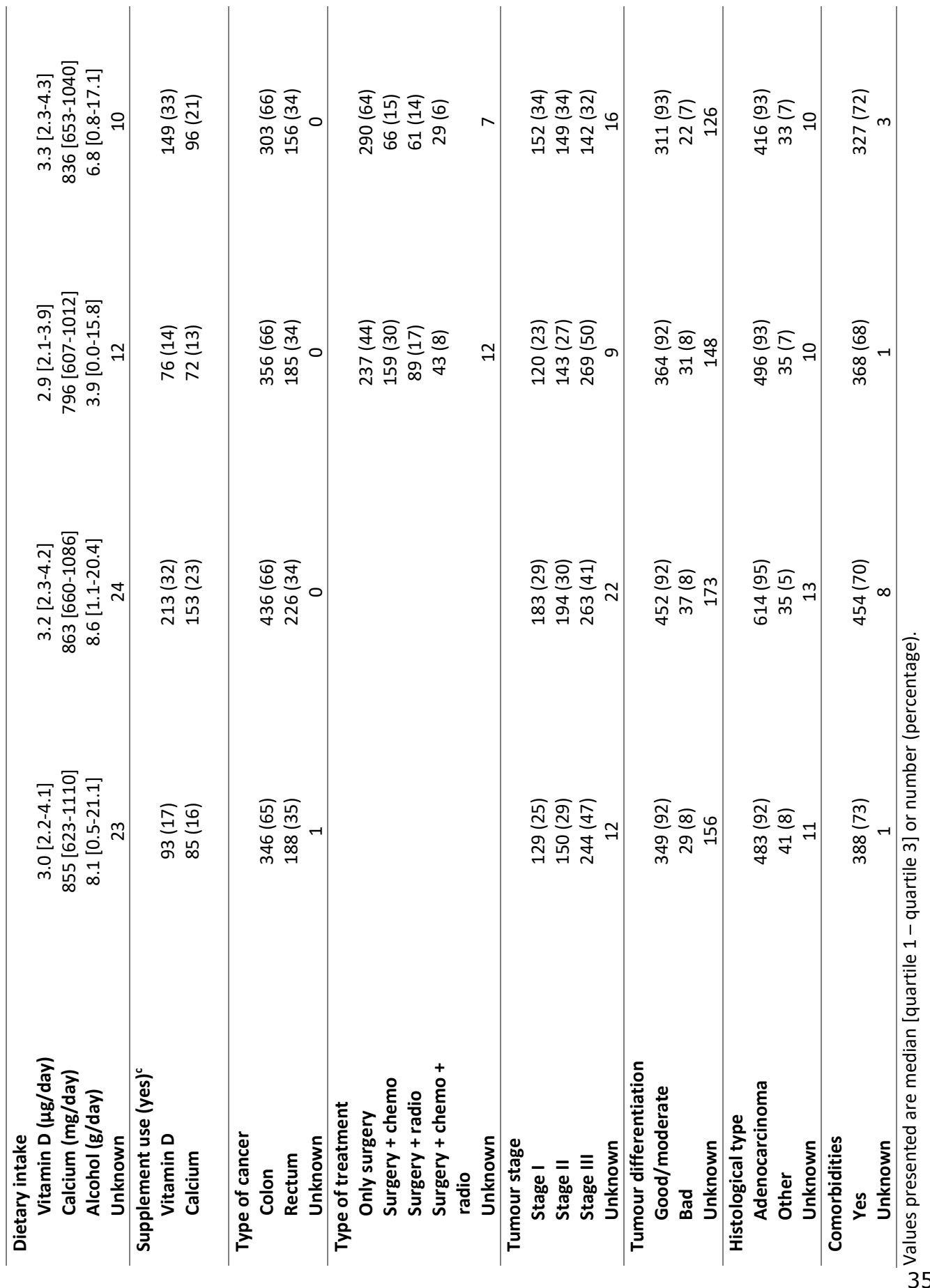


CHAPTER 2

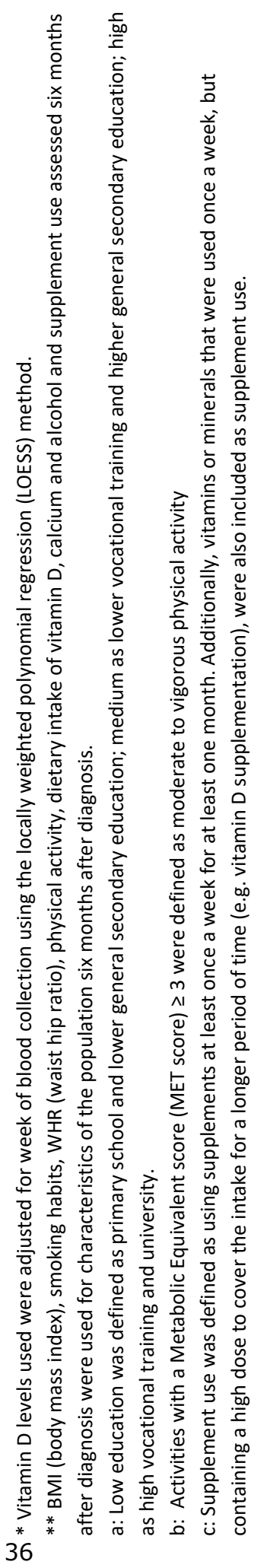




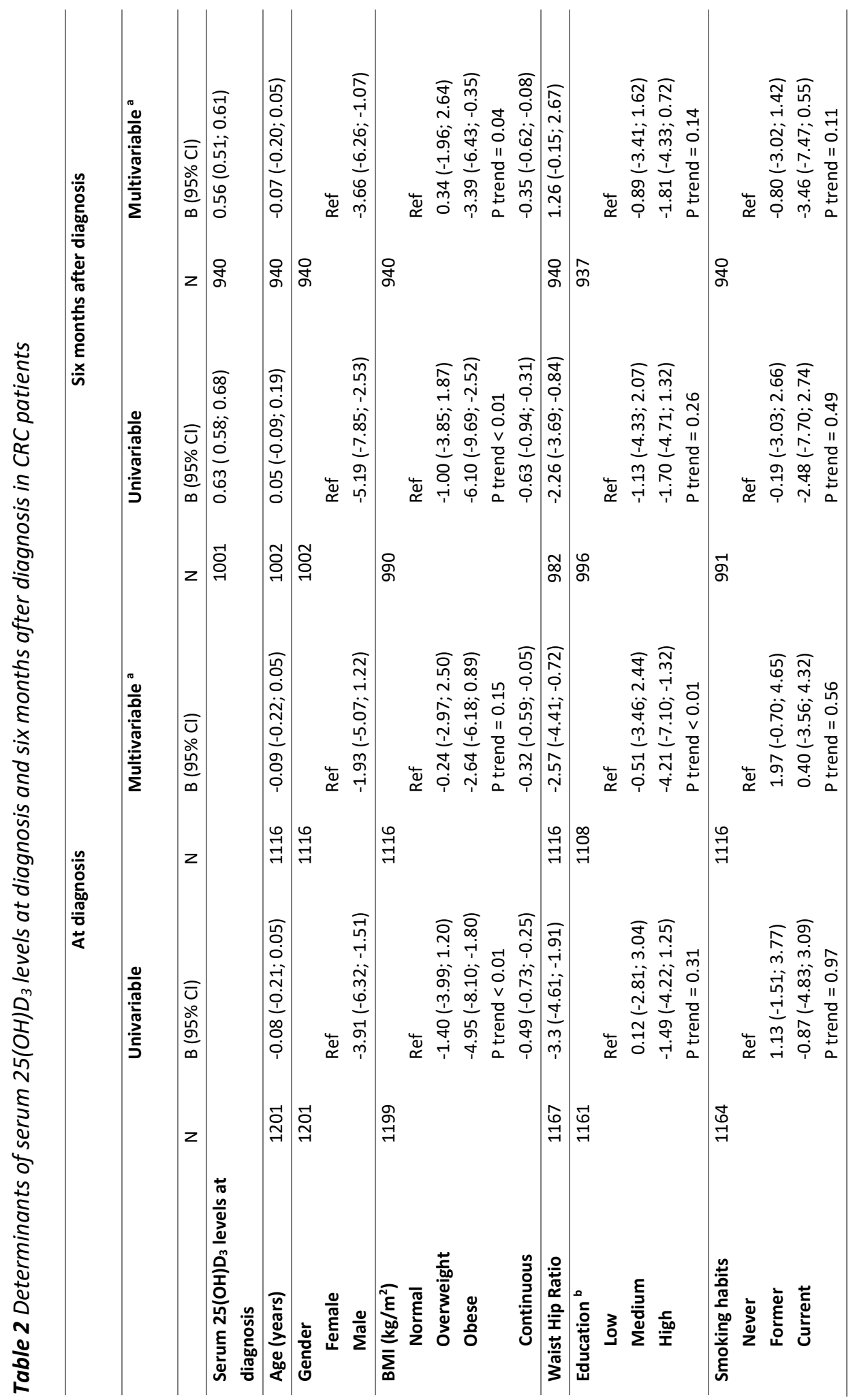




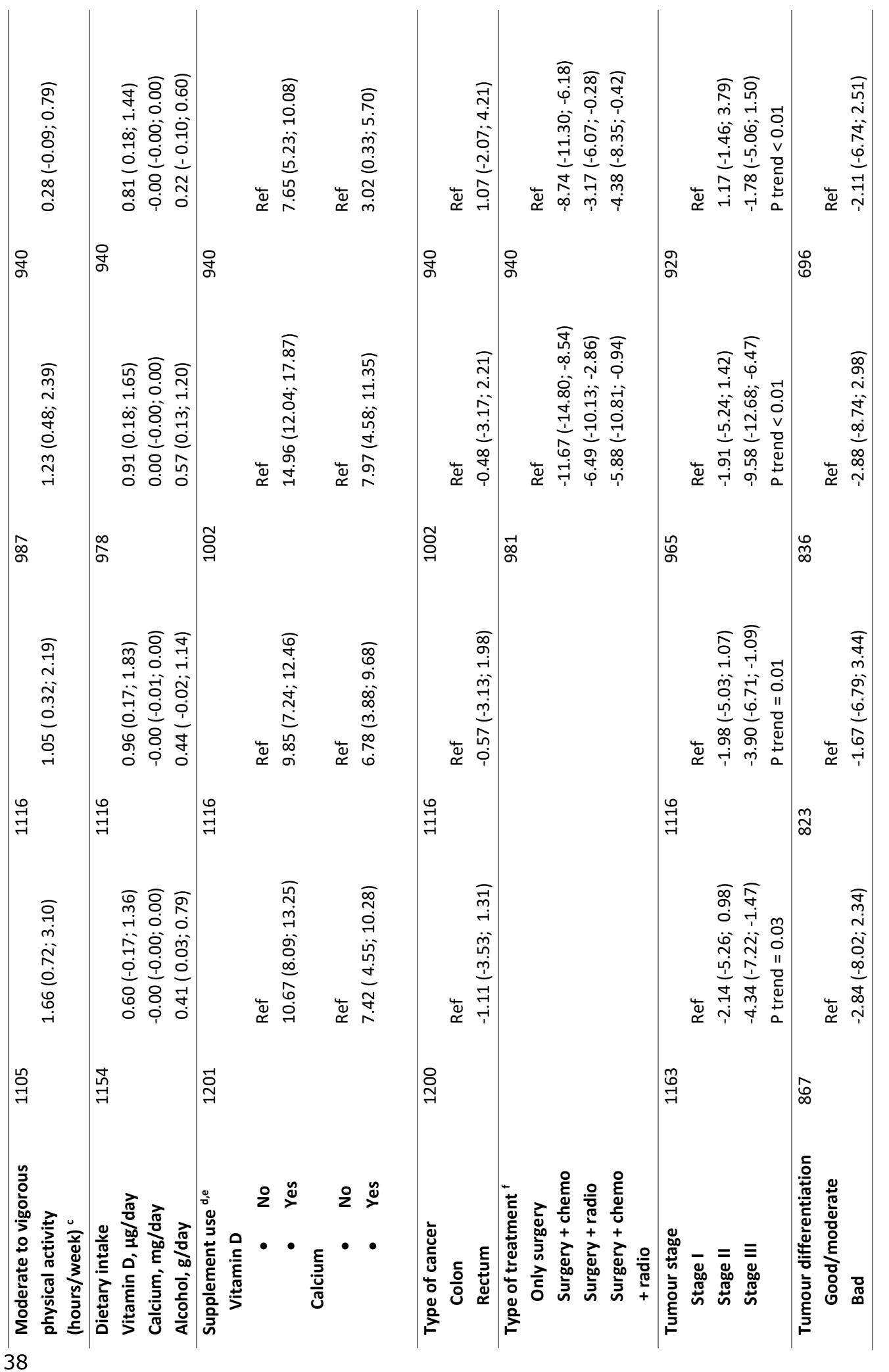




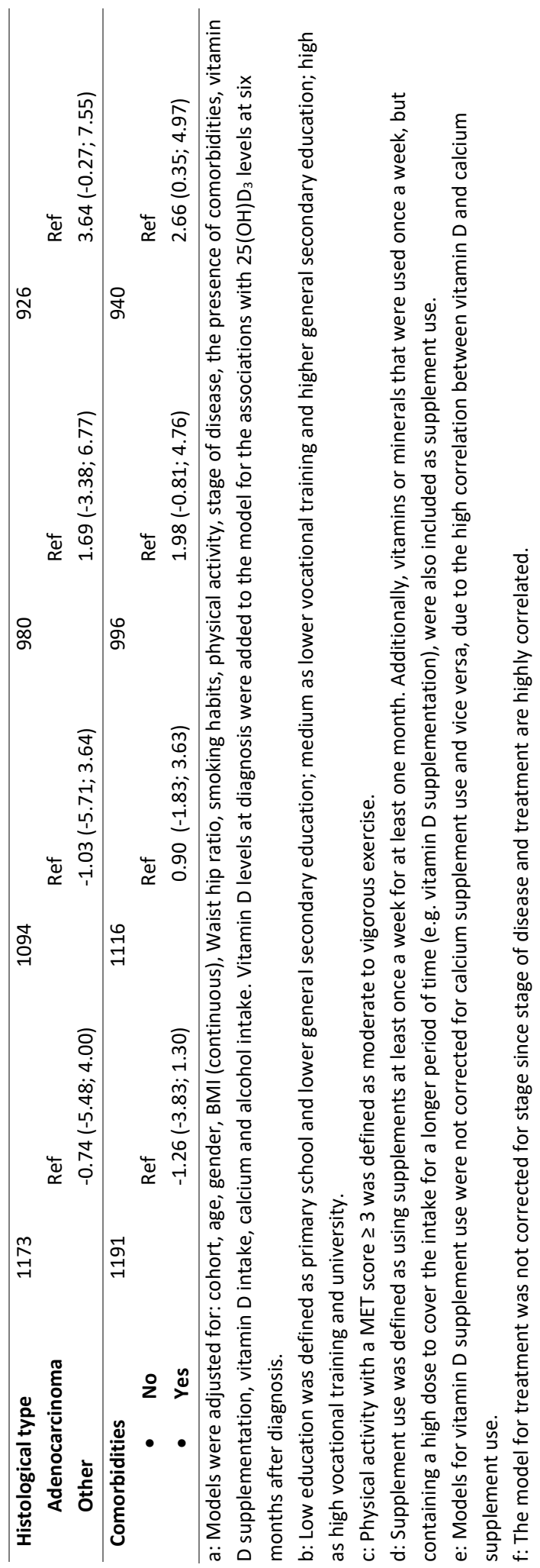




\section{DETERMINANTS OF CHANGES IN VITAMIN D LEVELS OVER TIME.}

After adjustment for other demographic and lifestyle factors $25(\mathrm{OH}) \mathrm{D}_{3}$ levels decreased $6.7 \mathrm{nmol} / \mathrm{L}(95 \% \mathrm{Cl}-9.8 ;-3.8)$ more in patients receiving chemotherapy compared to patients who underwent surgery only (table 3). Looking at absolute levels, median 25(OH) $\mathrm{D}_{3}$ levels were $55 \mathrm{nmol} / \mathrm{L}$ [IQR 42-70] at diagnosis and $52 \mathrm{nmol} / \mathrm{L}$ [IQR 41-68] six months after diagnosis in patients who only underwent surgery. For patients receiving chemotherapy median $25(\mathrm{OH}) \mathrm{D}_{3}$ levels were $53 \mathrm{nmol} / \mathrm{L}$ [IQR41-66] at diagnosis and 42 $\mathrm{nmol} / \mathrm{L}$ [IQR 31-53] six months after diagnosis.

In all patients $25(\mathrm{OH}) \mathrm{D}_{3}$ levels decreased over time, however, using vitamin $\mathrm{D}$ supplements six months after diagnosis, but not at diagnosis, was associated with a 4 $\mathrm{nmol} / \mathrm{L}$ less decrease in $25(\mathrm{OH}) \mathrm{D}_{3}$ levels $(95 \% \mathrm{Cl} 1.2 ; 6.8)$ compared to not using vitamin $\mathrm{D}$ supplements at both time points.

Table 3 Determinants of changes in vitamin $D$ levels between diagnosis and six months after diagnosis in CRC patients

\begin{tabular}{|c|c|c|c|c|}
\hline & $\mathbf{N}$ & $\begin{array}{l}\text { Estimated changes } \\
\text { (nmol/L) }(95 \% \mathrm{Cl}) \\
\text { Crude model }\end{array}$ & $\mathbf{N}$ & $\begin{array}{l}\text { Estimated changes }^{a} \\
\text { (nmol/L) }(95 \% \mathrm{Cl}) \\
\text { Adjusted model }\end{array}$ \\
\hline Age (years) & 1002 & $0.12(0.00 ; 0.25)$ & 955 & $-0.05(-0.19 ; 0.09)$ \\
\hline Gender & 1002 & & 955 & \\
\hline Female & & Ref & & Ref \\
\hline Male & & $-0.45(-2.78 ; 1.87)$ & & $-2.32(-5.33 ; 0.69)$ \\
\hline BMI $\left(\mathrm{kg} / \mathrm{m}^{2}\right)$ & 996 & & 954 & \\
\hline Normal & & Ref & & Ref \\
\hline Overweight & & $1.83(-0.82 ; 4.48)$ & & $0.61(-2.21 ; 3.43)$ \\
\hline Obese & & $0.34(-2.84 ; 3.52)$ & & $-1.14(-4.71 ; 2.43)$ \\
\hline Continuous & & $-0.01(-0.27 ; 0.26)$ & & $-0.16(-0.46 ; 0.14)$ \\
\hline WHR & 989 & $1.65(0.29 ; 3.01)$ & 955 & $1.40(-0.01 ; 2.82)$ \\
\hline Education $^{b}$ & 996 & & 952 & \\
\hline Low & & Ref & & Ref \\
\hline Medium & & $0.33(-2.28 ; 2.94)$ & & $-0.87(-3.73 ; 1.98)$ \\
\hline High & & $-0.68(-3.65 ; 2.29)$ & & $-0.03(-2.75 ; 2.70)$ \\
\hline Smoking habits & 994 & & 955 & \\
\hline Never & & Ref & & Ref \\
\hline Former & & $-0.81(-3.29 ; 1.68)$ & & $-1.29(-3.87 ; 1.29)$ \\
\hline Current & & $-2.35(-6.73 ; 2.03)$ & & $-3.08(-7.46 ; 1.32)$ \\
\hline Moderate to vigorous & 992 & & 955 & \\
\hline physical activity & & $-0.03(-0.14 ; 0.08)$ & & $-0.09(-0.53 ; 0.36)$ \\
\hline (hours/week) ${ }^{c}$ & & & & \\
\hline Dietary intake & 988 & & 955 & \\
\hline Vitamin D, $\mu \mathrm{g} /$ day & & $-0.22(-1.00 ; 0.55)$ & & $0.10(-0.70 ; 0.89)$ \\
\hline Calcium, mg/day & & $-0.00 ;(-0.00 ; 0.00)$ & & $0.00(-0.00 ; 0.01)$ \\
\hline Alcohol, g/day & & $-0.00(-0.30 ; 0.43)$ & & $-0.11(-0.39 ; 0.28)$ \\
\hline
\end{tabular}




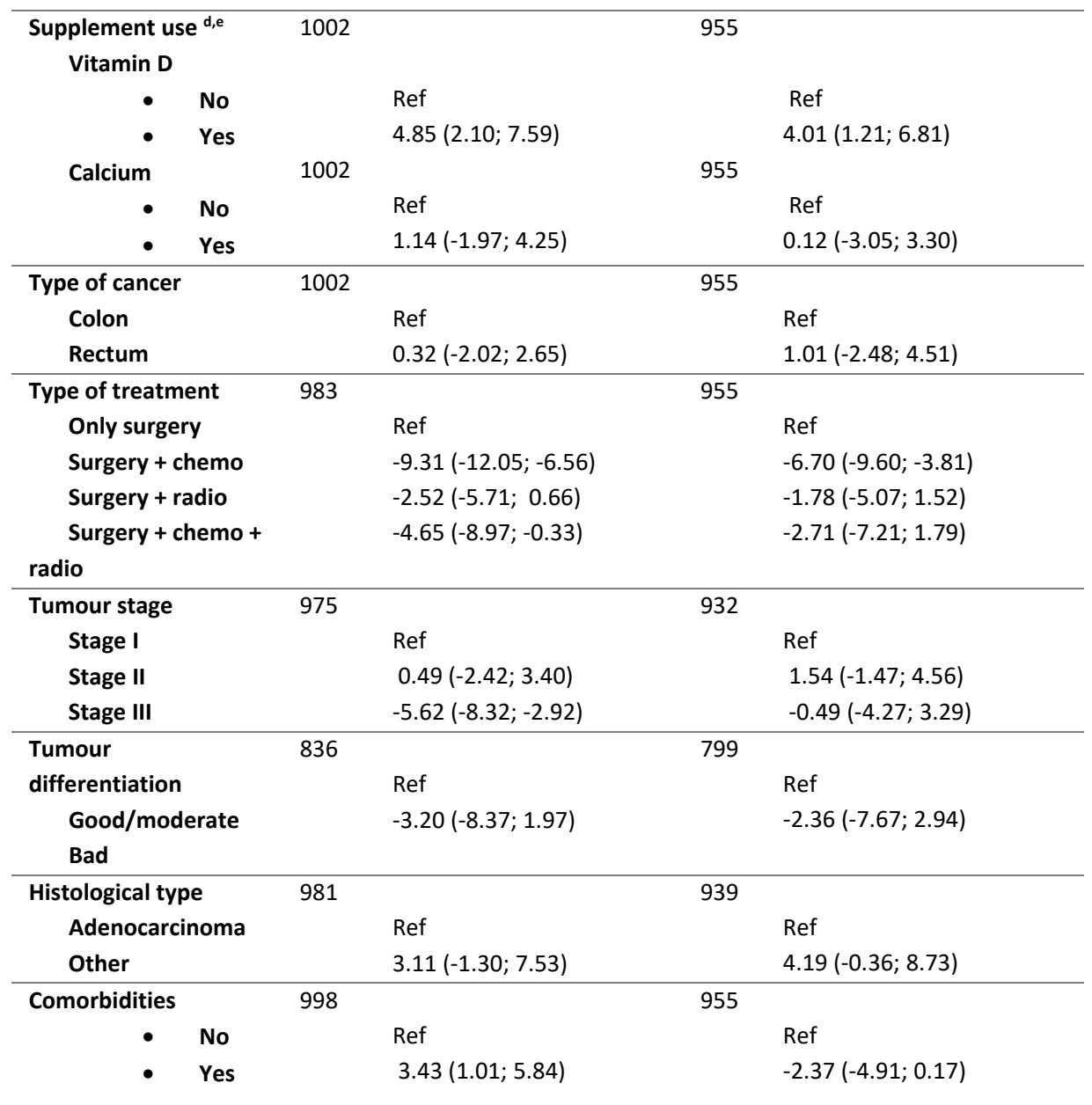

a: Models were adjusted for: cohort, age gender, BMI (continuous), Waist hip ratio, smoking habits, physical activity, vitamin D supplementation, vitamin D intake, calcium and alcohol intake. Type of treatment was added in the model for the associations six months after diagnosis.

b: Low education was defined as primary school and lower general secondary education; medium as lower vocational training and higher general secondary education; high as high vocational training and university. c: Physical activity with a MET score $\geq 3$ was defined as moderate to vigorous exercise. $\mathrm{d}$ : Supplement use was defined as using supplements at least once a week for at least one month. Additional, vitamins or minerals that were used once a week, but containing a high dose to cover the intake for a longer period of time (e.g. vitamin D supplementation), were also included as supplement use.

e: Models for vitamin D supplement use were not corrected for calcium supplement use and vice versa, due to the high correlation between vitamin $\mathrm{D}$ and calcium supplement use.

When only including patients of whom we know that blood was obtained before treatment commenced, determinants of 25(OH)D3 levels at diagnosis, at six months and of changes in $25(\mathrm{OH}) \mathrm{D}_{3}$ levels over time were similar as for the total study population (data not shown). 


\section{DISCUSSION}

We investigated determinants of $25(\mathrm{OH}) \mathrm{D}_{3}$ levels at diagnosis, of $25(\mathrm{OH}) \mathrm{D}_{3}$ at six months after diagnosis and of changes in $25(\mathrm{OH}) \mathrm{D}_{3}$ levels over time in newly diagnosed CRC patients. Several demographic, lifestyle and clinical factors were associated with $25(\mathrm{OH}) \mathrm{D}_{3}$ levels, of which the use of vitamin $D$ supplements and cancer treatment were found to be consistent determinants of $25(\mathrm{OH}) \mathrm{D}_{3}$ levels. However, it should be noted that both supplementation and receiving chemotherapy had a relatively low influence on $25(\mathrm{OH}) \mathrm{D}_{3}$ levels, an increase of approximately $4 \mathrm{nmol} / \mathrm{L}$ and a decrease of approximately $7 \mathrm{nmol} / \mathrm{L}$, respectively.

The median serum $25(\mathrm{OH}) \mathrm{D}_{3}$ level at $\mathrm{CRC}$ diagnosis in this study was 53 [41-67] nmol/L. Other studies measuring serum $25(\mathrm{OH}) \mathrm{D}_{3}$ levels at or before CRC diagnosis, found comparable levels (43-65 nmol/L) [3,5,8,9,19,27,28]. Six months after diagnosis we observed a median serum $25(\mathrm{OH}) \mathrm{D}_{3}$ level of 48 [37-63] nmol/L. No other published studies investigated $25(\mathrm{OH}) \mathrm{D}_{3}$ levels approximately six months after diagnosis, but studies in which 25(OH)D levels were measured shortly after surgery found much lower levels (25 $\mathrm{nmol} / \mathrm{L}$ ) compared to our study $[4,6,7]$. Thus $25(\mathrm{OH}) \mathrm{D}_{3}$ levels seems to change after cancer diagnosis, this change is likely depending on treatment phase.

Cancer treatment, especially chemotherapy, was associated with lower $25(\mathrm{OH}) \mathrm{D}_{3}$ levels six months after diagnosis. On top of that, receiving chemotherapy compared with receiving surgery only was associated with a stronger decrease in $25(\mathrm{OH}) \mathrm{D}_{3}$ levels between diagnosis and six months post diagnosis. In line with our results, the study of Fakih and colleagues observed a threefold higher risk of having vitamin D deficiency in CRC patients receiving chemotherapy compared to patients not receiving chemotherapy [26]. In addition, the study of Isenring and colleagues also reported a decrease in 25(OH)D levels in cancer patients receiving chemotherapy [29]. The lower $25(\mathrm{OH}) \mathrm{D}_{3}$ levels and/or the steeper decrease in $25(\mathrm{OH}) \mathrm{D}_{3}$ levels in patients receiving chemotherapy can be induced by changes in lifestyle due to chemotherapy or by chemotherapy directly. It is plausible that patients receiving chemotherapy participate less in outdoor activities and therefore experience less sun exposure and have lower $25(\mathrm{OH}) \mathrm{D}_{3}$ levels. It could also be that chemotherapy leads to changes in dietary habits, for example in a lower intake of fatty fish [40], an important source of dietary vitamin D. However, we adjusted for physical activity and dietary vitamin $\mathrm{D}$ intake in our analyses, and still observed an association between chemotherapy and lower circulating $25(\mathrm{OH}) \mathrm{D}_{3}$ levels. It could be that the absorption of vitamin $\mathrm{D}$ from diet and supplements is impaired due to subclinical mucositis induced by chemotherapy $[26,41]$. Although we find a decrease in $25(\mathrm{OH}) \mathrm{D} 3$ levels in patients receiving chemotherapy, we did not find a significant decrease in $25(\mathrm{OH}) \mathrm{D}_{3}$ levels in patients who only underwent surgery and in patients who received radiation therapy or a combination of radio- and chemotherapy. The most plausible 
explanation for this is the timing of treatment, at the 6 months timepoint patients just finished chemotherapy treatment, while radiotherapy, chemoradiation and surgery are already 3-4 months ago. It is reasonable that vitamin D levels were lower shortly after surgery, radiotherapy or chemoradiation, but re-establish over time. Apart from treatment, we also found an association between stage of disease and $25(\mathrm{OH}) \mathrm{D}_{3}$ levels at baseline. In line our results, the study of Churilla et al., found a higher prevalence of vitamin D deficiency in stage III of disease [42]. A potential explanation could be that a more advanced stage of disease and/or larger tumour size is accompanied with more inflammation [43], which may lead to lower $25(\mathrm{OH}) \mathrm{D}_{3}$ levels [44].

Next to clinical and treatment factors, also dietary factors and supplement use were associated with $25(\mathrm{OH}) \mathrm{D}_{3}$ levels in our analyses. As can be expected, the use of vitamin $\mathrm{D}$ supplements and dietary vitamin $\mathrm{D}$ intake were determinants of $25(\mathrm{OH}) \mathrm{D}_{3}$ levels in the present study and also in several other studies in the general population [20-23,30]. In addition, alcohol intake and intake of calcium supplements were associated with $25(\mathrm{OH}) \mathrm{D}_{3}$ levels in our population and also in the general healthy population [20-23,30]. In our population, alcohol intake was positively associated with $25(\mathrm{OH}) \mathrm{D}_{3}$ levels at baseline. The mechanism underlying the relationship between alcohol and $25(\mathrm{OH}) \mathrm{D}_{3}$ levels remains unclear. A possible hypothesis is that alcohol may suppress parathyroid hormone (PTH) secretion, resulting in a lower conversion of $25(\mathrm{OH}) \mathrm{D}_{3}$ to 1,25 -dihydroxyvitamin $\mathrm{D}$ [45]. Calcium supplement use was associated with higher $25(\mathrm{OH}) \mathrm{D}_{3}$ levels. An explanation for this is that a higher calcium supplemental intake inhibits PTH, resulting in higher $25(\mathrm{OH}) \mathrm{D}_{3}$ levels [46]. In addition, many calcium supplements also contain vitamin $D$, thereby increasing $25(\mathrm{OH}) \mathrm{D}_{3}$ levels. Of all dietary determinants, as could be expected, the use of vitamin D supplements was most consistently associated with $25(\mathrm{OH}) \mathrm{D}_{3}$ levels as it was associated with $25(\mathrm{OH}) \mathrm{D}_{3}$ levels at diagnosis and at six months after diagnosis but also with changes in $25(\mathrm{OH}) \mathrm{D}_{3}$ levels over time.

We further observed associations between $25(\mathrm{OH}) \mathrm{D}_{3}$ levels and other modifiable lifestyle factors, such as obesity and physical activity in our study population, which has also been found by several studies in the general population [20-23]. BMI and WHR, indirect measurements of body fatness and body fat distribution respectively, were associated with lower serum $25(\mathrm{OH}) \mathrm{D}_{3}$ levels. The lower $25(\mathrm{OH}) \mathrm{D}_{3}$ levels with higher BMI may be explained by the fact that vitamin $\mathrm{D}$ is fat-soluble. Higher body fat reduces the availability of circulating $25(\mathrm{OH}) \mathrm{D}_{3}$. In addition to body fatness, more time spent on moderate-tovigorous physical activity was associated with higher $25(\mathrm{OH}) \mathrm{D}_{3}$ levels, which is most likely due to outdoor activities that increase sun light exposure.

Although we observed a larger decrease in $25(\mathrm{OH}) \mathrm{D}_{3}$ levels in patients receiving chemotherapy, based on this study we cannot advice to give supplements to patients undergoing chemotherapy to counteract the decrease in $25(\mathrm{OH}) \mathrm{D}_{3}$ levels. It should be 
clear first whether these changes in $25(\mathrm{OH}) \mathrm{D}_{3}$ levels during treatment influence short and long term health effects, such as treatment efficacy, quality of life after treatment, cancer recurrence and cancer survival. Further studies should focus on the effects of changes in $25(\mathrm{OH}) \mathrm{D}_{3}$ levels on short- and long term health effects. Besides, results found in this study can provide potential relevant information to design intervention studies aimed to improve $25(\mathrm{OH}) \mathrm{D}_{3}$ levels e.g. which patients groups should be targeted and which dietary and lifestyle factors are important to intervene on.

The present study has some limitations. First, although sun exposure is the most important source of vitamin $D$, and thereby likely a strong determinant, it was not measured in a large part of the study population (COLON study, $\mathrm{N}=922$ ), and only briefly with one general question (How many days a week were you outside for at least 15 minutes with uncovered hands and face?) in the remaining part (EnCoRe study, n=279). Although we could not investigate sun exposure as a determinant of serum $25(\mathrm{OH}) \mathrm{D}_{3}$ levels, we did adjust for week of blood collection, by which we were able to obtain $25(\mathrm{OH}) \mathrm{D}_{3}$ levels independently of season of blood collection and thereby adjusted partly for sun exposure. In addition, we measured dietary intake using two different methods for part of our population. While an FFQ was used in the COLON study for both timepoints, an FFQ (first time-point) and a food diary (second time-point) was used in the EnCoRe study. Although only dietary vitamin D, calcium and alcohol intake were used in these analyses, assessing these by two different methods may have influenced the analysis of changes over time. However, sensitivity analyses in patients of the COLON study, which used one method for both time-points showed similar results regarding dietary vitamin $\mathrm{D}$, calcium and alcohol intake and its relation with $25(\mathrm{OH}) \mathrm{D}_{3}$ levels (data not shown). Last, some differences in patients' characteristics between the two cohorts were found. Compared to patients included in the COLON study, patients in the EnCoRe study had a higher BMI, had a higher vitamin D and calcium intake via the diet, were more likely to have a good to moderate tumour differentiation and were more likely to suffer from comorbidities at diagnosis. However, we adjusted for cohort in our analyses thereby correcting for these differences.

The present study also has important strengths. First of all, this is the largest study that prospectively assessed $25(\mathrm{OH}) \mathrm{D}_{3}$ levels before and after treatment in CRC patients. Our data extend existing evidence regarding lifestyle and treatment characteristics influencing $25(\mathrm{OH}) \mathrm{D}_{3}$ levels. Furthermore, we had data available on many clinical and lifestyle characteristics, making it possible to assess a wide range of lifestyle and clinical determinants that possibly could influence $25(\mathrm{OH}) \mathrm{D}_{3}$ levels.

In conclusion, the most consistent determinants of $25(\mathrm{OH}) \mathrm{D}_{3}$ levels in the first six months after diagnosis and of $25(\mathrm{OH}) \mathrm{D}_{3}$ changes over time in CRC patients were use of vitamin $\mathrm{D}$ supplements and type of colorectal cancer treatment, where the use of vitamin D 
supplement was associated with higher $25(\mathrm{OH}) \mathrm{D}_{3}$ levels and chemotherapy with lower $25(\mathrm{OH}) \mathrm{D}_{3}$ levels. In addition, the use of vitamin $\mathrm{D}$ supplements was associated with a smaller decrease in $25(\mathrm{OH}) \mathrm{D}_{3}$ levels or time compared to not using vitamin $\mathrm{D}$ supplements. Receiving chemotherapy compared to surgery only was associated with a stronger decrease in $25(\mathrm{OH}) \mathrm{D}_{3}$ levels over time. Knowing which determinants are associated with low $25(\mathrm{OH}) \mathrm{D}_{3}$ levels, will enable health professionals to identify patients at high risk of low $25(\mathrm{OH}) \mathrm{D}_{3}$ levels. Monitoring these patients and improving $25(\mathrm{OH}) \mathrm{D}_{3}$ levels when needed, can possibly result in better cancer prognosis. Further studies are needed to evaluate whether changes in $25(\mathrm{OH}) \mathrm{D}_{3}$ levels during treatment influences treatment efficacy, quality of life, cancer recurrence and survival. 


\section{ADDITIONAL INFORMATION:}

\section{FUNDING}

The COLON study was financially supported by Wereld Kanker Onderzoek Fonds (WKOF) \& World Cancer Research Fund International (WCRF International) as well as by funds from grant 2014/1179 as part of the World Cancer Research Fund International Regular Grant Programme; Alpe d'Huzes/Dutch Cancer Society (UM 2012-5653, UW 2013-5927, UW 2015-7946); and ERA-NET on Translational Cancer Research (TRANSCAN/Dutch Cancer Society: UW2013-6397, UW2014-6877 and the Netherlands Organization for Health Research and Development (ZonMw, the Netherlands). The EnCoRe study was supported by grants from the Stichting Alpe d'HuZes within the research program 'Leven met kanker' of the Dutch Cancer Society (Grant No. UM-2010-4867 and UM 2012-5653) and by a grant from Kankeronderzoekfonds Limburg as part of Health Foundation Limburg (Grant No. 00005739). Eline H. van Roekel was funded by the Wereld Kanker Onderzoek Fonds (WKOF), part of the World Cancer Research Fund International grant programme (grant number 2016/1620).

\section{AUTHORS' CONTRIBUTIONS}

E Wesselink, E Kampman, MJL Bours, HJW de Wilt, MP Weijenberg, RF Witkamp, R Winkels, DEG Kok and FJB van Duijnhoven contributed to the design of this study. $E$ Wesselink, E van Roekel, J van den Ouweland, M van Zutphen, $\mathrm{H}$ van Baar, AJMR Geijsen, M Snellen, HJW de Wilt, B Hansson, M Aquarius, SO Breukink and ETP Keulen contributed to recruitment of participants and the data collection. Statistical data analyses were done by $\mathrm{E}$ Wesselink. The manuscript was drafted by E Wesselink and FJB van Duijnhoven, and all authors critically read and revised the manuscript. All authors approved the final version of the manuscript.

\section{ACKNOWLEDGEMENTS}

The authors would like to thank the investigators at Wageningen University \& Research and the co-workers from the following hospitals for their involvement in recruitment for the COLON study: Hospital Gelderse Vallei, Ede; Radboudumc, Nijmegen; Slingeland Hospital, Doetinchem; Canisius Wilhelmina Hospital, Nijmegen; Rijnstate Hospital, Arnhem; Gelre Hospitals, Apeldoorn/Zutphen; Hospital Bernhoven, Uden; Isala, Zwolle; ZGT, Almelo; Martini Hospital, Groningen; Admiraal de Ruyter Hospital, Goes/Vlissingen. We would like to thank all participants of the EnCoRe study and the health professionals in the three hospitals involved in the recruitment of participants of the study: Maastricht University Medical Center, VieCuri Medical Center, and Zuyderland Medical Center. 


\section{REFERENCES}

[1] Miller KD, Siegel RL, Lin CC, Mariotto AB, Kramer JL, Rowland JH, Stein KD, Alteri R, Jemal A: Cancer treatment and survivorship statistics, 2016. CA: a cancer journal for clinicians 2016, 66:271-89.

[2] Maalmi H, Walter V, Jansen L, Boakye D, Schöttker B, Hoffmeister M, Brenner H: Association between Blood 25-Hydroxyvitamin D Levels and Survival in Colorectal Cancer Patients: An Updated Systematic Review and MetaAnalysis. Nutrients 2018, 10:896.

[3] Ng K, Meyerhardt JA, Wu K, Feskanich D, Hollis BW, Giovannucci EL, Fuchs CS: Circulating 25-hydroxyvitamin d levels and survival in patients with colorectal cancer. Journal of Clinical Oncology 2008, 26:2984-91.

[4] Mezawa H, Sugiura T, Watanabe M, Norizoe C, Takahashi D, Shimojima A, Tamez S, Tsutsumi Y, Yanaga K, Urashima M: Serum vitamin D levels and survival of patients with colorectal cancer: post-hoc analysis of a prospective cohort study. BMC Cancer 2010, 10:347.

[5] Fedirko V, Riboli E, Tjonneland A, Ferrari P, Olsen A, Bueno-de-Mesquita HB, van Duijnhoven FJB, Norat T, Jansen EHJM, Dahm CC, Overvad K, Boutron-Ruault M-C, Clavel-Chapelon F, Racine A, Lukanova A, Teucher B, Boeing H, Aleksandrova K, Trichopoulou A, Benetou V, Trichopoulos D, Grioni S, Vineis P, Panico S, Palli D, Tumino R, Siersema PD, Peeters PH, Skeie G, Brustad M, Chirlaque M-D, Barricarte A, Ramon Quiros J, Sanchez MJ, Dorronsoro M, Bonet C, Palmqvist R, Hallmans G, Key TJ, Crowe F, Khaw K-T, Wareham N, Romieu I, McKay J, Wark PA, Romaguera D, Jenab M: Prediagnostic 25-hydroxyvitamin D, VDR and CASR polymorphisms, and survival in patients with colorectal cancer in western European ppulations. Cancer epidemiology, biomarkers \& prevention : a publication of the American Association for Cancer Research, cosponsored by the American Society of Preventive Oncology 2012, 21:582-93.

[6] Zgaga L, Theodoratou E, Farrington SM, Din FV, Ooi LY, Glodzik D, Johnston S, Tenesa A, Campbell H, Dunlop MG: Plasma Vitamin D Concentration Influences Survival Outcome After a Diagnosis of Colorectal Cancer. Journal of Clinical Oncology 2014, 32:2430-9.

[7] Maalmi H, Walter V, Jansen L, Chang-Claude J, Owen RW, Ulrich A, Schottker B, Hoffmeister M, Brenner H: Relationship of very low serum 25-hydroxyvitamin D3 levels with long-term survival in a large cohort of colorectal cancer patients from Germany. European journal of epidemiology 2017, 32:961-71.

[8] Tretli S, Schwartz GG, Torjesen PA, Robsahm TE: Serum levels of 25-hydroxyvitamin D and survival in Norwegian patients with cancer of breast, colon, lung, and lymphoma: a population-based study. Cancer Causes Control 2012, 23:363-70.

[9] Ng K, Sargent DJ, Goldberg RM, Meyerhardt JA, Green EM, Pitot HC, Hollis BW, Pollak MN, Fuchs CS: Vitamin D status in patients with stage IV colorectal cancer: findings from Intergroup trial N9741. Journal of Clinical Oncology 2011, 29:1599-606.

[10] Keum N, Lee DH, Greenwood DC, Manson JE, Giovannucci E: Vitamin D supplementation and total cancer incidence and mortality: a meta-analysis of randomized controlled trials. Annals of Oncology 2019, 30:733-43. [11] Nowak A, Boesch L, Andres E, Battegay E, Hornemann T, Schmid C, Bischoff-Ferrari HA, Suter PM, Krayenbuehl P-A: Effect of vitamin D3 on self-perceived fatigue: A double-blind randomized placebo-controlled trial. Medicine 2016, 95.

[12] Anglin RE, Samaan Z, Walter SD, McDonald SD: Vitamin D deficiency and depression in adults: systematic review and meta-analysis. The British journal of psychiatry 2013, 202:100-7.

[13] Turner AG, Anderson PH, Morris HA: Vitamin D and bone health. Scandinavian Journal of Clinical and Laboratory Investigation 2012, 72:65-72.

[14] Reuss-Borst M, Hartmann U, Scheede C, Weiss J: Prevalence of osteoporosis among cancer patients in Germany. Osteoporosis international 2012, 23:1437-44.

[15] Arndt V, Merx H, Stegmaier C, Ziegler H, Brenner H: Restrictions in quality of life in colorectal cancer patients over three years after diagnosis: a population based study. European journal of cancer 2006, 42:1848-57.

[16] Stone P, Richardson A, Ream E, Smith A, Kerr D, Kearney N, Forum CF: Cancer-related fatigue: inevitable, unimportant and untreatable? Results of a multi-centre patient survey. Annals of Oncology 2000, 11:971-5.

[17] Jansen L, Koch L, Brenner H, Arndt V: Quality of life among long-term ( $\geqslant 5$ years) colorectal cancer survivors-Systematic review. European journal of cancer 2010, 46:2879-88.

[18] Holick MF: Vitamin D deficiency. New England Journal of Medicine 2007, 357:266-81.

[19] Wesa KM, Segal NH, Cronin AM, Sjoberg DD, Jacobs GN, Coleton MI, Fleisher M, Dnistrian AM, Saltz LB, Cassileth BR: Serum 25-hydroxy vitamin D and survival in advanced colorectal cancer: a retrospective analysis. Nutrition and cancer 2015, 67:424-30.

[20] Touvier M, Deschasaux M, Montourcy M, Sutton A, Charnaux N, Kesse-Guyot E, Assmann KE, Fezeu L, LatinoMartel P, Druesne-Pecollo N: Determinants of vitamin D status in Caucasian adults: influence of sun exposure, 
dietary intake, sociodemographic, lifestyle, anthropometric, and genetic factors. Journal of Investigative Dermatology 2015, 135:378-88.

[21] Van Dam RM, Snijder MB, Dekker JM, Stehouwer CD, Bouter LM, Heine RJ, Lips P: Potentially modifiable determinants of vitamin D status in an older population in the Netherlands: the Hoorn Study-. The American journal of clinical nutrition 2007, 85:755-61.

[22] Brock K, Huang W-Y, Fraser D, Ke L, Tseng M, Stolzenberg-Solomon R, Peters U, Ahn J, Purdue M, Mason R: Low vitamin $D$ status is associated with physical inactivity, obesity and low vitamin $D$ intake in a large US sample of healthy middle-aged men and women. The Journal of steroid biochemistry and molecular biology 2010, 121:462-6.

[23] Larose TL, Chen Y, Camargo CA, Langhammer A, Romundstad P, Mai X-M: Factors associated with vitamin D deficiency in a Norwegian population: the HUNT Study. Journal of epidemiology and community health 2014 68:165-70.

[24] Waldron JL, Ashby HL, Cornes MP, Bechervaise J, Razavi C, Thomas OL, Chugh S, Deshpande S, Ford C, Gama R: Vitamin D: a negative acute phase reactant. Journal of clinical pathology 2013, 66:620-2.

[25] Toriola AT, Nguyen N, Scheitler-Ring K, Colditz GA: Circulating 25-hydroxyvitamin D (25-OHD) levels and prognosis among cancer patients: a systematic review. Cancer epidemiology, biomarkers \& prevention : a publication of the American Association for Cancer Research, cosponsored by the American Society of Preventive Oncology 2014.

[26] Fakih MG, Trump DL, Johnson CS, Tian L, Muindi J, Sunga AY: Chemotherapy is linked to severe vitamin D deficiency in patients with colorectal cancer. Int J Colorectal Dis 2009, 24:219-24.

[27] Ng K, Venook AP, Sato K, Yuan C, Hollis BW, Niedzwiecki D, Ye C, Chang I-W, O'Neil BH, Innocenti F: Vitamin D status and survival of metastatic colorectal cancer patients: Results from CALGB/SWOG 80405 (Alliance).

American Society of Clinical Oncology, 2015.

[28] Freedman DM, Looker AC, Abnet CC, Linet MS, Graubard BI: Serum 25-hydroxyvitamin D and cancer mortality in the NHANES III study (1988-2006). Cancer Res 2010, 70:8587-97.

[29] Isenring EA, Teleni L, Woodman RJ, Kimlin MG, Walpole E, Karapetis CS, Shawgi S, Kichenadasse G, Marshall S, Koczwara B: Serum vitamin D decreases during chemotherapy: an Australian prospective cohort study. Asia Pacific journal of clinical nutrition 2018, 27:962.

[30] Lips P, Schoor NM, Jongh RT: Diet, sun, and lifestyle as determinants of vitamin D status. Annals of the New York Academy of Sciences 2014, 1317:92-8.

[31] Blanchard CM, Denniston MM, Baker F, Ainsworth SR, Courneya KS, Hann DM, Gesme DH, Reding D, Flynn T, Kennedy JS: Do adults change their lifestyle behaviors after a cancer diagnosis? American Journal of Health Behavior 2003, 27:246-56.

[32] Winkels RM, Heine-Broring RC, van Zutphen M, van Harten-Gerritsen S, Kok DE, van Duijnhoven FJ, Kampman E: The COLON study: Colorectal cancer: Longitudinal, Observational study on Nutritional and lifestyle factors that may influence colorectal tumour recurrence, survival and quality of life. BMC Cancer 2014, $14: 374$. [33] van Roekel EH, Bours MJ, de Brouwer CP, Ten Napel H, Sanduleanu S, Beets GL, Kant I, Weijenberg MP: The Applicability of the International Classification of Functioning, Disability, and Health to Study Lifestyle and Quality of Life of Colorectal Cancer Survivors. Cancer epidemiology, biomarkers \& prevention : a publication of the American Association for Cancer Research, cosponsored by the American Society of Preventive Oncology 2014, 23:1394-405.

[34] van den Ouweland JM, Beijers AM, van Daal H: Overestimation of 25-hydroxyvitamin D3 by increased ionisation efficiency of 3-epi-25-hydroxyvitamin D3 in LC-MS/MS methods not separating both metabolites as determined by an LC-MS/MS method for separate quantification of 25-hydroxyvitamin D3, 3-epi-25hydroxyvitamin D3 and 25-hydroxyvitamin D2 in human serum. Journal of Chromatography B 2014, 967:195-202. [35] Wendel-Vos GC, Schuit AJ, Saris WH, Kromhout D: Reproducibility and relative validity of the short questionnaire to assess health-enhancing physical activity. Journal of clinical epidemiology 2003, 56:1163-9.

[36] NEVO-table: Dutch Food Composition Table 2011/version 3. RIVM, Bilthoven 2011.

[37] Van Leersum NJ, Snijders HS, Henneman D, Kolfschoten NE, Gooiker GA, ten Berge MG, Eddes EH, Wouters MW, Tollenaar RA, Dutch Surgical Colorectal Cancer Audit G, Bemelman WA, van Dam RM, Elferink MA, Karsten TM, van Krieken JH, Lemmens VE, Rutten HJ, Manusama ER, van de Velde CJ, Meijerink WJ, Wiggers T, van der Harst E, Dekker JW, Boerma D: The Dutch surgical colorectal audit. European journal of surgical oncology : the journal of the European Society of Surgical Oncology and the British Association of Surgical Oncology 2013, 39:1063-70.

[38] Zhang H, Ahn J, Yu K: Comparing statistical methods for removing seasonal variation from vitamin D measurements in case-control studies. Statistics and its interface 2011, 4:85-93.

[39] Cleveland WS, Devlin SJ: Locally Weighted Regression: An Approach to Regression Analysis by Local Fitting. Journal of the American Statistical Association 1988, 83:596-610. 
[40] Daenen LG, Cirkel GA, Houthuijzen JM, Gerrits J, Oosterom I, Roodhart JM, van Tinteren H, Ishihara K, Huitema AD, Verhoeven-Duif NM: Increased plasma levels of chemoresistance-inducing fatty acid 16: 4 ( $n-3$ ) after consumption of fish and fish oil. JAMA oncology 2015, 1:350-8.

[41] Peterson DE, Bensadoun R-J, Roila F, Group ObotEGW: Management of oral and gastrointestinal mucositis: ESMO Clinical Practice Guidelines. Annals of Oncology 2011, 22:vi78-vi84.

[42] Churilla TM, Brereton HD, Klem M, Peters CA: Vitamin D deficiency is widespread in cancer patients and correlates with advanced stage disease: a community oncology experience. Nutrition and cancer 2012, 64:521-5. [43] Hanahan D, Weinberg RA: Hallmarks of cancer: the next generation. Cell 2011, 144:646-74.

[44] Mangin M, Sinha R, Fincher K: Inflammation and vitamin D: the infection connection. Inflammation Research 2014, 63:803-19.

[45] McCarty M, Thomas C: PTH excess may promote weight gain by impeding catecholamine-induced lipolysisimplications for the impact of calcium, vitamin D, and alcohol on body weight. Medical hypotheses 2003, 61:53542.

[46] Heaney RP: Vitamin D and calcium interactions: functional outcomes-. The American journal of clinical nutrition 2008, 88:541S-4S. 



\title{
CHAPTER 3
}

\section{VITAMIN D, MAGNESIUM, CALCIUM AND THEIR INTERACTION IN RELATION TO COLORECTAL CANCER RECURRENCE AND ALL- CAUSE MORTALITY}

\author{
Evertine Wesselink \\ Dieuwertje E Kok \\ Martijn J.L. Bours \\ Johannes H.W. de Wilt \\ Harm van Baar \\ Moniek van Zutphen \\ Anne M.J.R. Geijsen \\ Eric T.P. Keulen \\ Bibi M.E. Hansson \\ Jody van den Ouweland \\ Renger F. Witkamp \\ Matty P. Weijenberg \\ Ellen Kampman \\ Franzel J.B. van Duijnhoven
}

The American Journal of Clinical Nutrition 2020 May 1;111(5):1007-1017 


\section{ABSTRACT}

Background: Higher concentrations of 25-hydroxy vitamin $\mathrm{D}_{3}\left(25(\mathrm{OH}) \mathrm{D}_{3}\right)$ at diagnosis are associated with a lower mortality risk in colorectal cancer (CRC) patients. However, magnesium and calcium are important in vitamin D metabolism.

Objective: To investigate $25(\mathrm{OH}) \mathrm{D}_{3}$, magnesium or calcium and their interaction among patients with $\mathrm{CRC}$ in relation to recurrence and all-cause mortality.

Design: The study population included 1,169 newly-diagnosed stage I-III CRC patients from two prospective cohorts. Associations between $25(\mathrm{OH}) \mathrm{D}_{3}$ concentrations, magnesium or calcium intake through diet and/or supplements at diagnosis and recurrence and all-cause mortality were evaluated using multivariable Cox proportional hazard models. The interaction between $25(\mathrm{OH}) \mathrm{D}_{3}$ and magnesium or calcium was assessed by investigating 1) joint versus separate effects, using a single reference category, and 2) the effect estimates of one factor across strata of another.

Results: Serum $25(\mathrm{OH}) \mathrm{D}_{3}$, calcium and magnesium, alone and their interactions, were not associated with recurrence. Serum $25(\mathrm{OH}) \mathrm{D}_{3}$ concentrations seems to be associated with all-cause mortality. An inverse association between magnesium intake (HRQ3vs Q1 0.55, $95 \% \mathrm{Cl} 0.32,0.95$ and $\mathrm{HR}_{\mathrm{Q} 4 \text { vs } \mathrm{Q} 1} 0.65,95 \% \mathrm{Cl} 0.35,1.21$ ), but not calcium intake, and allcause mortality was observed. When investigating the interaction between $25(\mathrm{OH}) \mathrm{D}_{3}$ and magnesium, we observed the lowest risk of all-cause mortality in patients with sufficient vitamin D concentrations ( $\geq 50 \mathrm{nmol} / \mathrm{L}$ ) and a high magnesium intake (median split) (HR $0.53,95 \% \mathrm{Cl} 0.31,0.89$ ) compared to patients who were vitamin $\mathrm{D}$ deficient $(<50 \mathrm{nmol} / \mathrm{L})$ and had a low magnesium intake. No interactions between calcium and vitamin $D$ in relation to all-cause mortality were observed.

Conclusion: Our findings suggest that the presence of an adequate status of $25(\mathrm{OH}) \mathrm{D}_{3}$ in combination with an adequate magnesium intake is essential in lowering the risk of mortality in CRC patients, yet the underlying mechanism should be studied. In addition, diet and lifestyle intervention studies are needed to confirm our findings.

Keywords: colorectal cancer patients, $25(\mathrm{OH}) \mathrm{D}_{3}$, interactions, magnesium, calcium, recurrence, all-cause mortality 


\section{INTRODUCTION}

Evidence is accumulating that circulating vitamin D concentrations are inversely associated with mortality in colorectal cancer (CRC) patients [1-11]. Recently, Maalmi and colleagues performed a meta-analysis, including 11 original studies with a total of 7,718 CRC patients [12]. Pooled effect estimates comparing the highest versus lowest category of circulating 25-hydroxyvitamin $\mathrm{D}\left(25(\mathrm{OH}) \mathrm{D}_{3}\right)$ showed a hazard ratio $(\mathrm{HR})$ of 0.68 (95\% confidence interval $(\mathrm{Cl}) 0.55,0.85)$ for all-cause mortality and $0.67(95 \% \mathrm{Cl} 0.57,0.78)$ for CRC-specific mortality [12]. Although recurrence of the disease is a concern for CRC survivors[13] and a contributor to morbidity and mortality in CRC survivors [14], the association between $25(\mathrm{OH}) \mathrm{D}_{3}$ concentrations and $\mathrm{CRC}$ recurrence has hardly been reported so far.

Magnesium plays crucial roles in several biochemical processes involved in the synthesis and metabolism of vitamin $D$ [15]. The enzymatic conversion of $25(\mathrm{OH}) \mathrm{D}_{3}$ to $1,25(\mathrm{OH}) \mathrm{D}_{3}$, the active form of vitamin $D$, is magnesium dependent $[16,17]$. Vitamin $D$ resistant rickets, in which patients do not respond to vitamin $D$ supplementation, could be reversed by magnesium supplementation [18]. In addition, a previous cohort study in the general population observed a stronger inverse association between $25(\mathrm{OH}) \mathrm{D}_{3}$ concentrations and all-cause mortality in participants with a high magnesium intake (median >264 mg/day) compared to participants with a low magnesium intake ( $<264 \mathrm{mg} /$ day) [15]. When investigating magnesium alone, a borderline statistically significant inverse association between magnesium intake and all-cause mortality was observed in a meta-analysis of six prospective cohort studies among the general population ( $\mathrm{HR}$ highest vs lowest $0.88,95 \% \mathrm{Cl}$ 0.76-1.01). Whether magnesium, alone or in interaction with vitamin $D$, is also beneficial for patients with CRC is unknown.

Besides magnesium, calcium is also involved in vitamin D metabolism. A low calcium intake causes a high turnover of vitamin D metabolites, resulting in vitamin D deficiency, while a high calcium intake is vitamin D sparing [19]. Previously, a high post-diagnostic calcium intake was associated with a lower risk of all-cause mortality in CRC patients [20, 21]. Moreover, three previous randomized controlled trials in patients with colorectal adenomas showed a reduced adenoma recurrence with high-dose calcium supplementation (pooled relative risk $0.80,95 \% \mathrm{Cl} 0.68-0.93$ )[22]. On the contrary, another large randomized controlled trial observed no associations between high dose calcium and/or vitamin D supplementation and the risk of recurrent adenomas and even a higher risk of sessile serrated adenomas [23, 24]. Until now, however, it is unknown whether calcium intake is associated with CRC recurrence, especially in interaction with vitamin D concentrations.

The aim of our study was to investigate our hypothesis that higher vitamin D concentrations, magnesium intake and calcium intake, at diagnosis, are associated with a 
lower risk of recurrence and all-cause mortality in CRC patients. Above that, given the importance of magnesium and calcium in vitamin D metabolism, the interaction between vitamin $\mathrm{D}$ concentrations and magnesium intake or calcium intake in relation to CRC recurrence and all-cause mortality was investigated.

\section{METHODS}

\section{STUDY DESIGN}

The designs of the COLON study [25] (NCT03191110; clinical trials.gov) and the EnCoRe study [26] (NTR7099; trialregister.nl) have been described earlier. Briefly, newly diagnosed CRC patients were recruited directly after diagnosis in 14 hospitals and were followed during and after treatment from 2010 (COLON) or 2012 (EnCoRe) onwards. Men and women above the age of 18 were eligible. In the COLON study, patients with stage I-IV CRC were eligible. In the EnCoRe study, patients with stage IV of disease were not recruited. Non-Dutch speaking patients, (partial) bowel resection, chronic inflammatory bowel disease, hereditary CRC syndromes (e.g. Lynch syndrome, Familial Adenomatous Polyposis, Peutz-Jegher), dementia or another mental condition obstructing participation were excluded in both studies. The COLON study was approved by the Committee on Research involving Human Subjects, region Arnhem-Nijmegen, the Netherlands (2009349). The EnCoRe study was approved by the Medical Ethics Committee of the University Hospital Maastricht and Maastricht University, the Netherlands (METC 11-3-075). All patients signed informed consent.

Blood samples were available for 1,169 patients, $71 \%$ of all recruited participants. Patients with stage IV disease $(n=90)$ or with unknown stage $(n=37)$ were excluded from the analyses (Figure 1).

\section{BLOOD COLLECTION AND MEASUREMENT OF 25(OH) D $_{3}$ CONCENTRATIONS}

For the COLON study, blood samples were obtained in the hospital at diagnosis. In 93\% of the patients included in these analyses, blood was collected before the start of treatment. For the EnCoRe study, blood samples at diagnosis were obtained in the hospital or by a research assistant during a home visit before start of treatment. For both studies, blood samples were collected in a serum tube, centrifuged and aliquots were immediately stored at $-80^{\circ} \mathrm{C}$ until further analysis.

For both cohorts, serum $25(\mathrm{OH}) \mathrm{D}_{3}$ concentrations were measured by isotope-dilution liquid chromatography tandem mass spectrometry (LC-MS/MS) in the Canisius Wilhelmina Hospital, Nijmegen, the Netherlands [27]. The inter-assay coefficients of variation were $7.4 \%, 4.0 \%$ and $3.1 \%$ at $25(\mathrm{OH}) \mathrm{D}_{3}$ concentrations of $36.0,88$ and $124 \mathrm{nmol} / \mathrm{L}$, respectively. 
Serum $25(\mathrm{OH}) \mathrm{D}_{3}$ is the main circulating form of vitamin $\mathrm{D}$ and generally considered the most reliable measurement of an individual's vitamin D status [28].

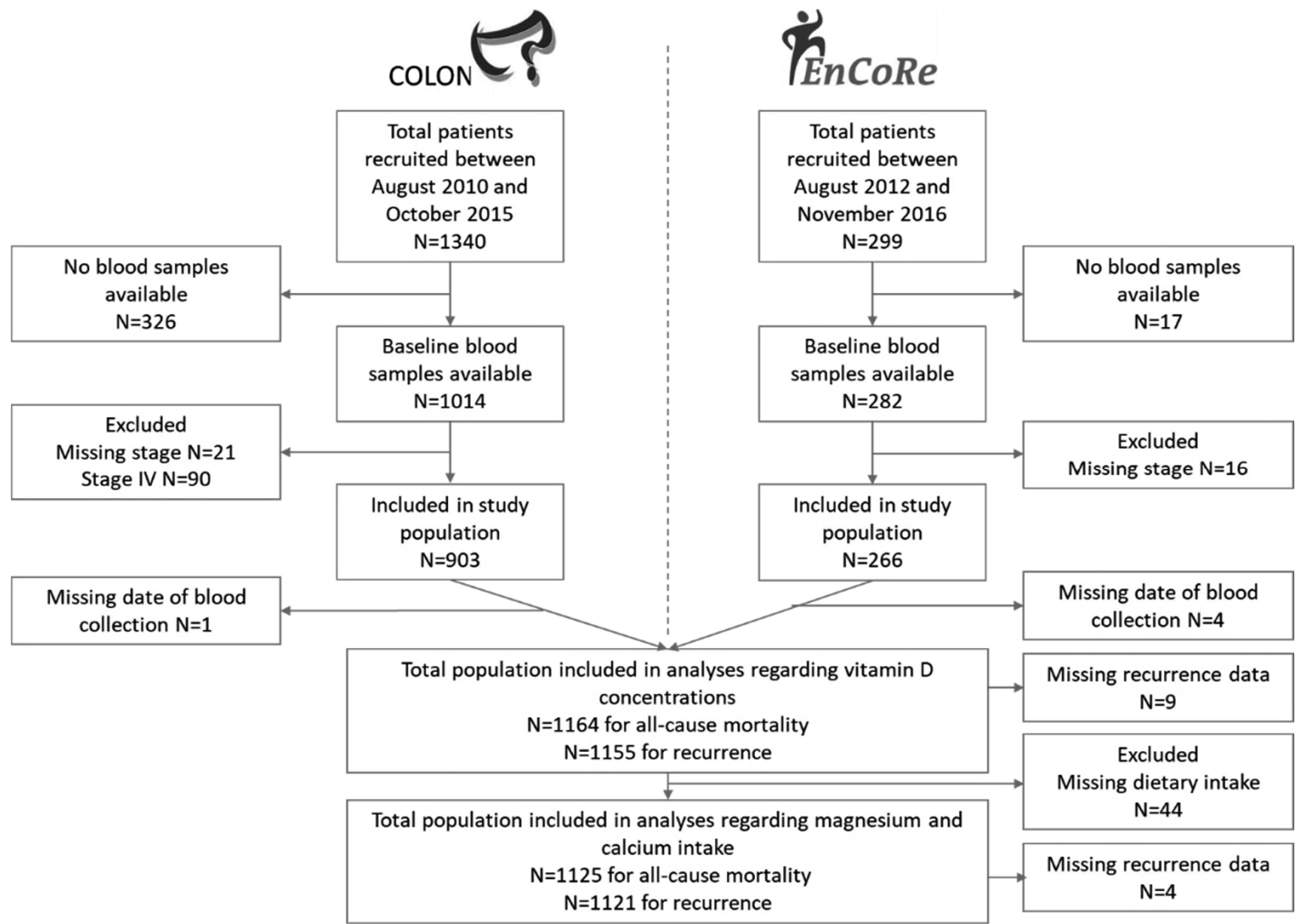

Figure 1: Flowchart representing patient selection for the current study.

\section{Data Collection}

Habitual dietary intake in the month (COLON study) or year (EnCoRe study) preceding diagnosis was assessed using an extended semi-quantitative food frequency questionnaire. The validated FFQ used in the COLON study consists of 204 items. The FFQ used in the EnCoRe study consists of 253 items and is recently validated for macro and micronutrients [29]. Dietary intake of vitamin D, magnesium and calcium was calculated for each food item based on frequency of intake, number of portions and portion size, as well as the type of product (e.g. whole grain or brown bread). Average daily vitamin D $(\mu \mathrm{g} /$ day), magnesium (mg/day) and calcium (g/day) intakes were calculated by adding all items containing the respective nutrient using data from the 2011 Dutch food composition table [30]. In the COLON study, supplement use was assessed by a dietary supplement questionnaire developed by the Division of Human Nutrition and Health of Wageningen University \& Research [25]. The dietary supplement questionnaire provided at time of diagnosis contains questions on use of multivitamin/minerals supplements and on the 
dosage and frequency of their intake. In the EnCoRe study, supplement use was assessed in detail by a research dietician during a home visit, using standardized forms, to record type and brand name of supplements, as well as frequency and duration of use, dosage, and ingredients (recorded from the package if necessary). For both studies, supplement use was defined as using supplements at least once a week for at least one month during the preceding year. Additionally, vitamins or minerals that were used once a month, but contained a high dose to cover the intake for a longer period (e.g. D-CURE $25.000 \mathrm{IE}$ Cholecalciferol supplementation), were also classified as supplement use. Supplement dosage per day was calculated using frequency of intake (e.g. once a week, every day), number of supplements and dosage per supplement. Total intake of vitamin D, magnesium or calcium was calculated by summing dietary intake and intake from dietary supplements.

Information on demographics (age, gender, education) and smoking habits was obtained using self-administered questionnaires in both cohorts at the same time as the blood samples were collected. Information on height, weight, and waist and hip circumference was collected using self-administered questionnaires in the COLON study. In the EnCoRe study, these measurements were performed by trained research dieticians during home visits. Physical activity was assessed using the Short QUestionnaire to ASsess Healthenhancing physical activity (SQUASH) in both cohorts [31].

Clinical data, such as stage of disease, tumour location (colon/rectum), date of start treatment, type of treatment (surgery, neo-adjuvant/adjuvant chemotherapy, radiation therapy) and presence of comorbidities (amongst others: diabetes, endocrine disorders, cardiovascular, gastro-intestinal) were derived from the Dutch ColoRectal Audit (DCRA) (COLON) and hospital records (EnCoRe). The DCRA is a nationwide audit initiated by the association of surgeons of the Netherlands to monitor, evaluate and improve CRC care [32].

\section{STUDY ENDPOINTS}

Information on recurrence was collected from medical records by the Dutch Cancer Registration. Recurrence is defined as a loco-regional recurrence or distant metastasis. Information on all-cause mortality was gathered from linkage with the Municipal Personal Record Database.

Follow-up time for recurrence was calculated starting from the date of blood collection until date of recurrence or until the date recurrence status was updated (February 2018 for the COLON study and March 2018 for the EnCoRe study) or until the date of end of follow-up, whichever came first. Follow-up time for all-cause mortality was defined starting from date of blood collection until date of death, or until the last date vital status 
was updated $\left(25^{\text {th }}\right.$ of June 2019 for the COLON study and $20^{\text {th }}$ of May 2019 for the EnCoRe study), or until the date of end of follow-up, whichever came first.

\section{Data ANALYses}

Patient characteristics at diagnosis were described as numbers with percentages or medians with interquartile range (IQR) for the total study population and stratified by vitamin D status (deficiency = serum $25(\mathrm{OH}) \mathrm{D}_{3}<50 \mathrm{nmol} / \mathrm{L}$ and sufficiency = serum $\left.25(\mathrm{OH}) \mathrm{D}_{3} \geq 50 \mathrm{nmol} / \mathrm{L}\right)$ [33]. Patients with missing data in the main exposure variables ( $n=343$ for vitamin $D$ concentrations and $n=44$ for dietary intake) were excluded from analyses (Figure 1). Descriptive statistics were used to assess differences in characteristics between patients with missing exposure data and those without missing exposure data. Correlations between magnesium, calcium and vitamin D intake and concentrations were assessed using Pearson correlation coefficients.

The association between serum $25(\mathrm{OH}) \mathrm{D}_{3}$ concentrations and $\mathrm{CRC}$ recurrence as well as all-cause mortality were assessed using multivariable Cox proportional hazard models. Serum $25(\mathrm{OH}) \mathrm{D}_{3}$ concentrations were entered in the model continuously per $10 \mathrm{nmol} / \mathrm{L}$ and based on clinically defined cut-off points [33] (severely deficient $<30 \mathrm{nmol} / \mathrm{L}$; deficient 30-49 nmol/L; sufficient 50-74 nmol/L (reference); optimal $\geq 75 \mathrm{nmol} / \mathrm{L}$ ).

The association between magnesium and calcium intake and CRC recurrence and all-cause mortality was also examined using multivariable Cox proportional hazard models. Cohortspecific quartiles of intake were calculated, since slightly different food frequency questionnaires were used in the two cohorts. To test for linearity among quartiles of magnesium and calcium intake, $\mathrm{p}$ values for trend were calculated by including the quartiles as a continuous variable in the model. Analyses were performed for dietary intake of magnesium and calcium as well as for total intake (diet and supplements).

First a crude model, including only the main exposure of interest and the outcome was performed. Second, based on literature, the following covariates were added to the multivariable models investigating the association between vitamin $D$, magnesium and calcium individually: age (continuous), sex (male/female), stage $(\mathrm{I}, \mathrm{II}, \mathrm{III})$, tumour location (colon/rectal), BMI (continuous), moderate-vigorous physical activity (continuous, hours / week), season of blood collection (spring, summer, autumn, winter; only in the model for vitamin D), total energy intake (quartiles) $[12,15]$ and cohort. Additionally, other potential confounders were tested and included in the model when the HR changed by more than $10 \%$. Smoking, education level, having comorbidities at diagnosis $(y / n)$, the use of statins $(y / n)$, use of proton pump inhibitors $(y / n)$ and alcohol intake $(g / d a y)$ did not influence the HR and were thus not included in the models. Finally, in a third model we also added the nutrients involved in vitamin D metabolism, to get more insight in how 
each nutrient individually, independently of the others, was associated with recurrence and mortality. Thus, magnesium (quartiles) and calcium (quartiles) intake were added to the models of vitamin D. Calcium and vitamin D concentrations were added to the models of magnesium. Magnesium and vitamin D concentrations were added to the models of calcium.

Log-transformed curves were used for visual inspection of the assumption for the Cox proportional hazard model. No strong evidence of non-parallelism of the log-log curves was observed.

We investigated interaction using two different methods, as recommended by Knol and VanderWeele [34] 1) by investigating the joint versus separate effects of $25(\mathrm{OH}) \mathrm{D}_{3}$ concentrations and magnesium or calcium intake using one reference category (vitamin D deficient as well as low magnesium or low calcium intake), and 2) by investigating the effect estimate of one factor across strata of another factor. A median split was used to define high and low magnesium and calcium intake. Both additive and multiplicative interactions were investigated. Interaction on the additive scale was investigated, as assessing additive rather than multiplicative interaction can help determine which subgroups would benefit most from an increase in vitamin D and/or magnesium or calcium [34, 35]. To investigate interaction on an additive scale the relative excess risk due to interaction (RERI) was calculated: RERI=HR vitD-Mg- $-\mathrm{HR}_{\text {vitD }+M g-}-\mathrm{HR}_{\text {vitD-Mg+ }}+1$ [35]. Thus, the HR found for the combined exposure (VitD $\geq 50 \mathrm{nmol} / \mathrm{L}$ and $\mathrm{Mg}>$ median) were compared with the HRs for each of the two exposures alone. Because the RERI was developed for risk factors rather than preventive factors, the group with the lowest risk was used as reference category (i.e. vitamin D concentrations $\geq 50 \mathrm{nmol} / \mathrm{L}$ and magnesium and calcium intake $>$ median). A RERI of zero means no additive interaction, a RERI $<0$ a negative additive interaction and a RERI $>0$ a positive additive interaction. The $p$ for multiplicative interaction was calculated by adding vitamin D status, magnesium or calcium intake as well as the product term of vitamin D status * magnesium or calcium intake to the model.

In a sensitivity analyses, patients who donated blood after the start of treatment $(n=76)$ were excluded.

Statistical analyses were performed in SAS 9.4 (SAS Institute, Cary NC). P-values $<0.05$ were considered statistically significant.

\section{RESULTS}

In total, 1,169 CRC patients recruited between August 2010 and November 2016 with stage I-III were included in the present analyses (Figure 1), 903 (77\%) from the COLON study and 266 (23\%) from the EnCoRe study. Median age was 67.0 [IQR 61.7-72.9] years 
and 418 (36\%) participants were female (Table 1). Two-thirds of the patients had colon cancer. Almost half of the patients had stage III disease. Around $25 \%$ of the patients used vitamin D containing supplements, $21 \%$ used calcium containing supplements and $19 \%$ used magnesium-containing supplements. Patients who had sufficient vitamin D concentrations ( $\geq 50 \mathrm{nmol} / \mathrm{L}$ ) were more often female, were more physically active and used more often vitamin $\mathrm{D}$, calcium and magnesium supplements compared to patients who had deficient concentrations (<50nmol/L) (Table 1).

No differences were observed between patients who donated blood and patients who did not. Patients of whom no dietary data was available, seemed to be slightly older and had more often advanced disease and more often comorbidities (data not shown). Magnesium intake and calcium intake were moderately correlated $(r=0.6)$. A moderate correlation between vitamin $D$ intake and magnesium or calcium intake was observed ( $r=0.4)$. Vitamin $D$ concentrations were not linearly correlated with magnesium or calcium intake $(r=0.1)$.

During a median follow-up of 3.5 [IQR 2.5-4.7] years for recurrence and 4.7 [IQR 4.0-6.2] years for all-cause mortality, 155 recurrences and 191 deaths occurred. Almost half (42\%) of the patients died after a recurrence. The total follow up time was 4084 years for recurrence and 5769 years for all-cause mortality.

Table 1 Baseline characteristics of stage I-III colorectal cancer patients, overall and stratified by vitamin D status

\begin{tabular}{|c|c|c|c|}
\hline & $\begin{array}{l}\text { Total population } \\
(n=1169)\end{array}$ & $\begin{array}{l}\text { Deficient } \\
(25(\mathrm{OH}) \mathrm{D}<50 \mathrm{nmol} / \mathrm{L}) \\
(\mathrm{n}=500)\end{array}$ & $\begin{array}{l}\text { Sufficient } \\
(25(\mathrm{OH}) \mathrm{D} \geq 50 \mathrm{nmol} / \mathrm{L}) \\
(\mathrm{n}=669)\end{array}$ \\
\hline $\begin{array}{l}\text { Serum } 25(\mathrm{OH}) \mathrm{D}_{3} \\
\text { concentrations } \\
\text { (nmol/L) }\end{array}$ & $54.5[39.8-70.2]$ & $37.3[28.4-43.9]$ & $67.4[58.9-79.6]$ \\
\hline \multicolumn{4}{|l|}{$\begin{array}{l}\text { Season of blood } \\
\text { collection }^{1}\end{array}$} \\
\hline Spring & $295(25)$ & $162(33)$ & $133(20)$ \\
\hline Summer & $327(28)$ & $69(14)$ & 258 (39) \\
\hline Autumn & $268(23)$ & $103(21)$ & $165(25)$ \\
\hline Winter & 274 (24) & 164 (33) & 110 (17) \\
\hline Unknown & 5 & 2 & 3 \\
\hline Age (years) & $67.0[61.7-72.9]$ & $67.0[60.9-74.4]$ & $66.9[62.3-72.4]$ \\
\hline Gender (female) & $418(36)$ & $164(33)$ & $254(38)$ \\
\hline BMI $\left(\mathrm{kg} / \mathrm{m}^{2}\right)$ & $26.3[24.1-29.3]$ & $26.8[24.2-29.8]$ & $26.1[24.1-29.0]$ \\
\hline Unknown & 2 & 0 & 2 \\
\hline \multicolumn{4}{|l|}{ Education $^{2}$} \\
\hline Low & $542(49)$ & $225(48)$ & 317 (49) \\
\hline Medium & $260(23)$ & $105(22)$ & $155(24)$ \\
\hline High & $314(28)$ & $142(30)$ & $172(27)$ \\
\hline Unknown & 53 & 28 & 25 \\
\hline
\end{tabular}




\begin{tabular}{|c|c|c|c|}
\hline \multicolumn{4}{|l|}{ Smoking habits } \\
\hline Current & $141(12)$ & $58(12)$ & $83(13)$ \\
\hline Former & 665 (29) & $278(57)$ & 387 (59) \\
\hline Never & 329 (29) & $148(31)$ & $181(28)$ \\
\hline Unknown & 34 & 16 & 18 \\
\hline Physical activity ${ }^{3}$ & $10.5[5.0-19.5]$ & $8.7[4.0-17.5]$ & $12.0[5.9-20.5]$ \\
\hline \multicolumn{4}{|l|}{ (hours/week) } \\
\hline unknown & 36 & 16 & 20 \\
\hline \multicolumn{4}{|l|}{ Dietary intake } \\
\hline Vitamin D ( $\mu \mathrm{g} /$ day) & $3.1[2.2-4.2]$ & $3.1[2.2-4.0]$ & $3.2[2.3-4.3]$ \\
\hline Calcium (mg/day) & 861 [639-1094] & 862 [630-1109] & 859 [648-1087] \\
\hline Magnesium & 318 [257-384] & 316 [252-381] & 321 [259-387] \\
\hline (mg/day) & $8.1[0.8-20.5]$ & $6.1[0.4-19.4]$ & $8.9[1.3-20.7]$ \\
\hline Alcohol (g/day) & 44 & 20 & 24 \\
\hline \multicolumn{4}{|l|}{ Unknown } \\
\hline \multicolumn{4}{|l|}{ Supplement use (yes) } \\
\hline Vitamin D & $289(25)$ & $76(16)$ & $213(33)$ \\
\hline Calcium & $238(21)$ & $80(16)$ & $158(24)$ \\
\hline Magnesium & $226(19)$ & $81(16)$ & $145(22)$ \\
\hline \multicolumn{4}{|l|}{ Type of cancer } \\
\hline Colon & $768(66)$ & $320(64)$ & $448(67)$ \\
\hline Rectum & $401(34)$ & $180(36)$ & $221(33)$ \\
\hline \multicolumn{4}{|l|}{ Tumour stage } \\
\hline I & $312(27)$ & $115(23)$ & $197(29)$ \\
\hline II & $346(30)$ & $148(30)$ & $198(30)$ \\
\hline III & $511(44)$ & 237 (47) & $274(41)$ \\
\hline \multicolumn{4}{|l|}{ Comorbidities } \\
\hline Yes & $285(71)$ & $361(72)$ & $464(70)$ \\
\hline Unknown & 8 & 1 & 7 \\
\hline
\end{tabular}

Values presented are median [quartile 1 - quartile 3] or number (percentage).

1: Spring: March-May; Summer: June-August; Autumn: September-November; Winter: December-February.

2: Low education was defined as primary school and lower general secondary education; medium as lower vocational training and higher general secondary education; high as high vocational training and university.

3: Activities with a Metabolic Equivalent score (MET score) $\geq 3$ were defined as moderate to vigorous physical activity

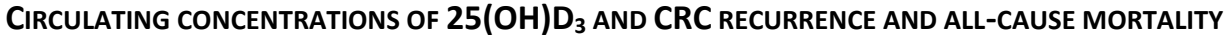

No association between $25(\mathrm{OH}) \mathrm{D}_{3}$ concentrations at diagnosis and $\mathrm{CRC}$ recurrence was observed (Table 2). Severe vitamin D deficiency (<30 nmol/L) compared to sufficient concentrations (50-74 $\mathrm{nmol} / \mathrm{L})$ tends to be associated with a higher risk of all-cause mortality (HR 1.46, 95\% Cl 0.92, 2.32; P for trend 0.08). 


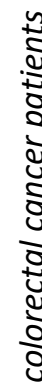

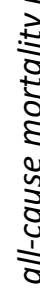

产

c)

¿ับ

tons

$\sqrt{n}$

is

ț

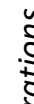

$\frac{8}{8}$

空

รั้

4

कू
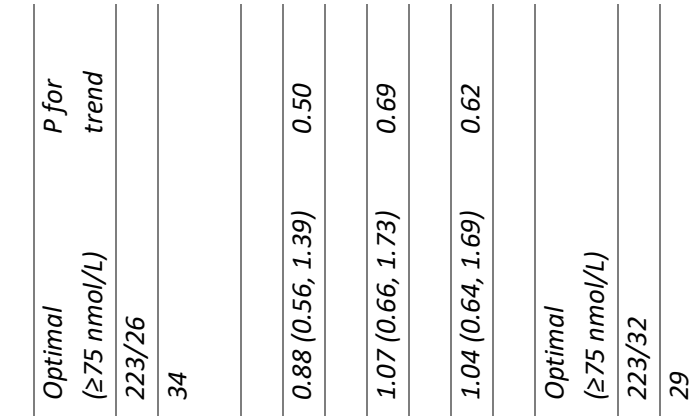

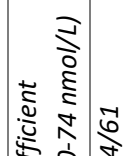

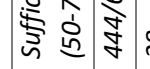
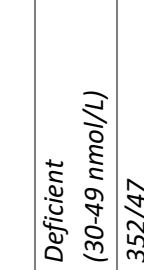

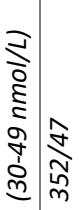

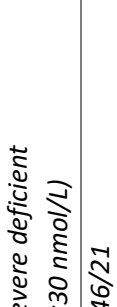

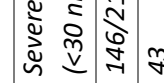

है

๕ั ฐั

ปั้

䓀

ฏิ

䋿

言

(4)

望

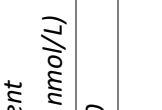

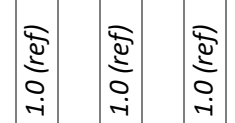

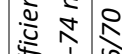

等道量的

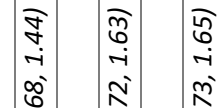

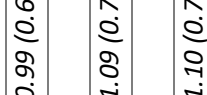

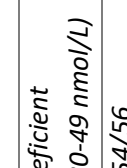

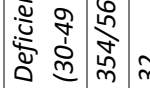

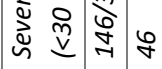

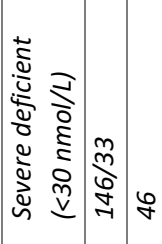

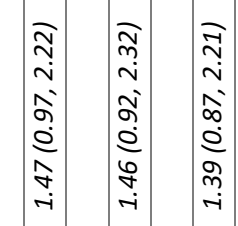

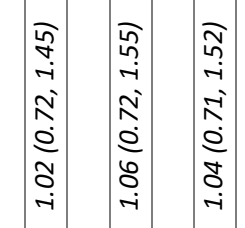

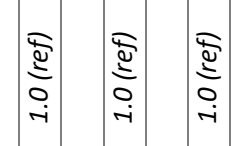




\section{MAGNESIUM OR CALCIUM INTAKE AND CRC RECURRENCE AND ALL-CAUSE MORTALITY}

No associations between dietary as well as total magnesium intake and CRC recurrence were found (Table 3). An inverse association between magnesium intake (dietary as well as total intake) and all-cause mortality was found ( $H_{R_{Q 3 v s} Q_{1}} 0.48,95 \% \mathrm{Cl} 0.29,0.82$ and $\mathrm{HR}_{\mathrm{Q} 4 \text { vs } \mathrm{Q} 1} 0.55,95 \% \mathrm{Cl} 0.31,0.98 ; \mathrm{p}$ for trend $=0.02$ for total intake). After adjustment for $25(\mathrm{OH}) \mathrm{D}_{3}$ concentrations and calcium intake this association was attenuated (HR $\mathrm{H}_{\mathrm{Q} 3 \mathrm{v}} \mathrm{Q}$ 1 $0.55,95 \% \mathrm{Cl} 0.32,0.95$ and $\mathrm{HR}_{\mathrm{Q} 4}$ vs $\mathrm{Q}_{1} 0.65,95 \% \mathrm{Cl} 0.35,1.21 ; \mathrm{p}$ for trend $=0.11$ for total intake) (Table 3).

No associations between dietary as well as total calcium intake and CRC recurrence were observed (Table 4). An inverse association between total, but not dietary, calcium intake and all-cause mortality was observed ( $\mathrm{HR}_{\mathrm{Q} 4}$ vs $\mathrm{Q}_{1} 0.58,95 \% \mathrm{Cl} 0.34,0.98 ; \mathrm{p}$ for trend 0.07 ). However, after adjustment for magnesium and $25(\mathrm{OH}) \mathrm{D}_{3}$ concentrations, this association was attenuated and no longer statistically significant $\left(\mathrm{HR}_{\mathrm{Q} 4}\right.$ vs $\mathrm{Q}_{1} 0.70,95 \% \mathrm{Cl} 0.40,1.21 ; \mathrm{p}$ for trend 0.27).

Table 3 Association of dietary and total magnesium intake at diagnosis with recurrence and all-cause mortality in colorectal cancer patients

\begin{tabular}{|c|c|c|c|c|c|}
\hline \multicolumn{6}{|c|}{ Dietary magnesium intake } \\
\hline & \multicolumn{5}{|c|}{ CRC recurrence } \\
\hline & Quartile 1 & Quartile 2 & Quartile 3 & Quartile 4 & $\begin{array}{l}P \text { for } \\
\text { trend }\end{array}$ \\
\hline No./events & $280 / 34$ & $281 / 36$ & $280 / 35$ & $280 / 40$ & \\
\hline $\begin{array}{l}\text { Events/1000 person } \\
\text { years }\end{array}$ & 35 & 35 & 35 & 43 & \\
\hline \multicolumn{6}{|l|}{ Model 1} \\
\hline HR (95\% Cl) & 1.0 (ref) & $1.02(0.64,1.63)$ & $1.01(0.63,1.61)$ & $1.20(0.76,1.89)$ & 0.47 \\
\hline \multicolumn{6}{|l|}{ Model 2} \\
\hline $\mathrm{HR}(95 \% \mathrm{Cl})$ & 1.0 (ref) & $1.19(0.72,1.97)$ & $1.10(0.61,2.01)$ & $1.38(0.66,2.87)$ & 0.70 \\
\hline \multicolumn{6}{|l|}{ Model 3} \\
\hline \multirow[t]{3}{*}{ HR (95\%Cl) } & 1.0 (ref) & $1.25(0.74,2.08)$ & $1.20(0.64,2.26)$ & $1.56(0.71,3.46)$ & 0.40 \\
\hline & \multicolumn{5}{|c|}{ All-cause mortality } \\
\hline & Quartile 1 & Quartile 2 & Quartile 3 & Quartile 4 & $\begin{array}{l}P \text { for } \\
\text { trend }\end{array}$ \\
\hline No./events & $280 / 56$ & $282 / 42$ & $282 / 34$ & $281 / 42$ & \\
\hline $\begin{array}{l}\text { Events/1000 person } \\
\text { years }\end{array}$ & 41 & 29 & 24 & 31 & \\
\hline \multicolumn{6}{|l|}{ Model 1} \\
\hline HR (95\% Cl) & 1.0 (ref) & $0.71(0.48,1.06)$ & $0.59(0.38,0.90)$ & $0.76(0.51,1.13)$ & 0.10 \\
\hline \multicolumn{6}{|l|}{ Model 2} \\
\hline HR (95\%Cl) & 1.0 (ref) & $0.65(0.42,1.00)$ & $0.46(0.26,0.79)$ & $0.51(0.26,0.98)$ & 0.04 \\
\hline \multicolumn{6}{|l|}{ Model 3} \\
\hline HR (95\%Cl) & 1.0 (ref) & $0.69(0.44,1.09)$ & $0.52(0.29,0.93)$ & $0.59(0.29,1.20)$ & 0.15 \\
\hline
\end{tabular}




\begin{tabular}{|c|c|c|c|c|c|}
\hline \multicolumn{6}{|c|}{ Total magnesium intake (Diet and supplements) } \\
\hline & \multicolumn{5}{|c|}{ CRC recurrence } \\
\hline & Quartile 1 & Quartile 2 & Quartile 3 & Quartile 4 & $\begin{array}{l}P \text { for } \\
\text { trend }\end{array}$ \\
\hline No./events & $280 / 34$ & $281 / 34$ & $280 / 37$ & $280 / 40$ & \\
\hline $\begin{array}{l}\text { Events/1000 person } \\
\text { years }\end{array}$ & 35 & 33 & 38 & 42 & \\
\hline \multicolumn{6}{|l|}{ Model 1} \\
\hline HR (95\% Cl) & 1.0 (ref) & $0.96(0.60,1.55)$ & $1.07(0.67,1.70)$ & $1.18(0.75,1.86)$ & 0.42 \\
\hline \multicolumn{6}{|l|}{ Model 2} \\
\hline HR (95\%Cl) & 1.0 (ref) & $1.15(0.69,1.90)$ & $1.20(0.69,2.11)$ & $1.39(0.77,2.53)$ & 0.24 \\
\hline \multicolumn{6}{|l|}{ Model 3} \\
\hline \multirow[t]{3}{*}{$\mathrm{HR}(95 \% \mathrm{Cl})$} & 1.0 (ref) & $1.19(0.72,1.99)$ & $1.32(0.74,2.36)$ & $1.57(0.84,2.92)$ & 0.13 \\
\hline & \multicolumn{5}{|c|}{ All-cause mortality } \\
\hline & Quartile 1 & Quartile 2 & Quartile 3 & Quartile 4 & $\begin{array}{l}\text { P for } \\
\text { trend }\end{array}$ \\
\hline No./events & $280 / 55$ & $282 / 46$ & $282 / 36$ & $281 / 37$ & \\
\hline $\begin{array}{l}\text { Events/1000 person } \\
\text { years }\end{array}$ & 41 & 33 & 26 & 27 & \\
\hline \multicolumn{6}{|l|}{ Model 1} \\
\hline HR (95\% Cl) & 1.0 (ref) & $0.80(0.54,1.18)$ & $0.63(0.41,0.95)$ & $0.66(0.40,1.00)$ & 0.02 \\
\hline \multicolumn{6}{|l|}{ Model 2} \\
\hline HR $(95 \% \mathrm{Cl})$ & 1.0 (ref) & $0.77(0.50,1.19)$ & $0.48(0.29,0.82)$ & $0.55(0.31,0.98)$ & 0.02 \\
\hline \multicolumn{6}{|l|}{ Model 3} \\
\hline HR (95\%Cl) & 1.0 (ref) & $0.83(0.53,1.28)$ & $0.55(0.32,0.95)$ & $0.65(0.35,1.21)$ & 0.11 \\
\hline
\end{tabular}

Quartiles of intake were cohort-specific:

Dietary intake of magnesium: COLON quartile 1 < 246 mg/day; quartile 2 246-305 mg/day; quartile 3 306-371 mg/day; quartile 4 > $371 \mathrm{mg} /$ day. EnCoRe quartile $1<300 \mathrm{mg} /$ day; quartile 2 300-364 mg/day; quartile 3 364$429 \mathrm{mg} /$ day; quartile $4>429 \mathrm{mg} /$ day

Total intake of magnesium: COLON quartile $1<258$ mg/day; quartile 2 258-322 mg/day; quartile $3323-398$ mg/day; quartile 4 > 398 mg/day. EnCoRe quartile $1<315$ mg/day; quartile 2 315-383 mg/day; quartile 3 384$463 \mathrm{mg} /$ day; quartile $4>464 \mathrm{mg} /$ day

Model 1: crude Cox proportional hazard model.

Model 2: adjusted for age, sex, stage, BMI, physical activity (moderate to vigorous in hours/week), tumour location, cohort and total energy intake.

Model 3: as model 2 and additionally adjusted for dietary calcium and vitamin D concentrations for the dietary intake models and total calcium and vitamin D concentrations for total intake (diet and supplements) models. $P$ values for trend were calculated by including the quartiles as a continuous variable in the model. 
Table 4 Association of dietary and total calcium intake at diagnosis with recurrence and all-cause mortality in colorectal cancer patients

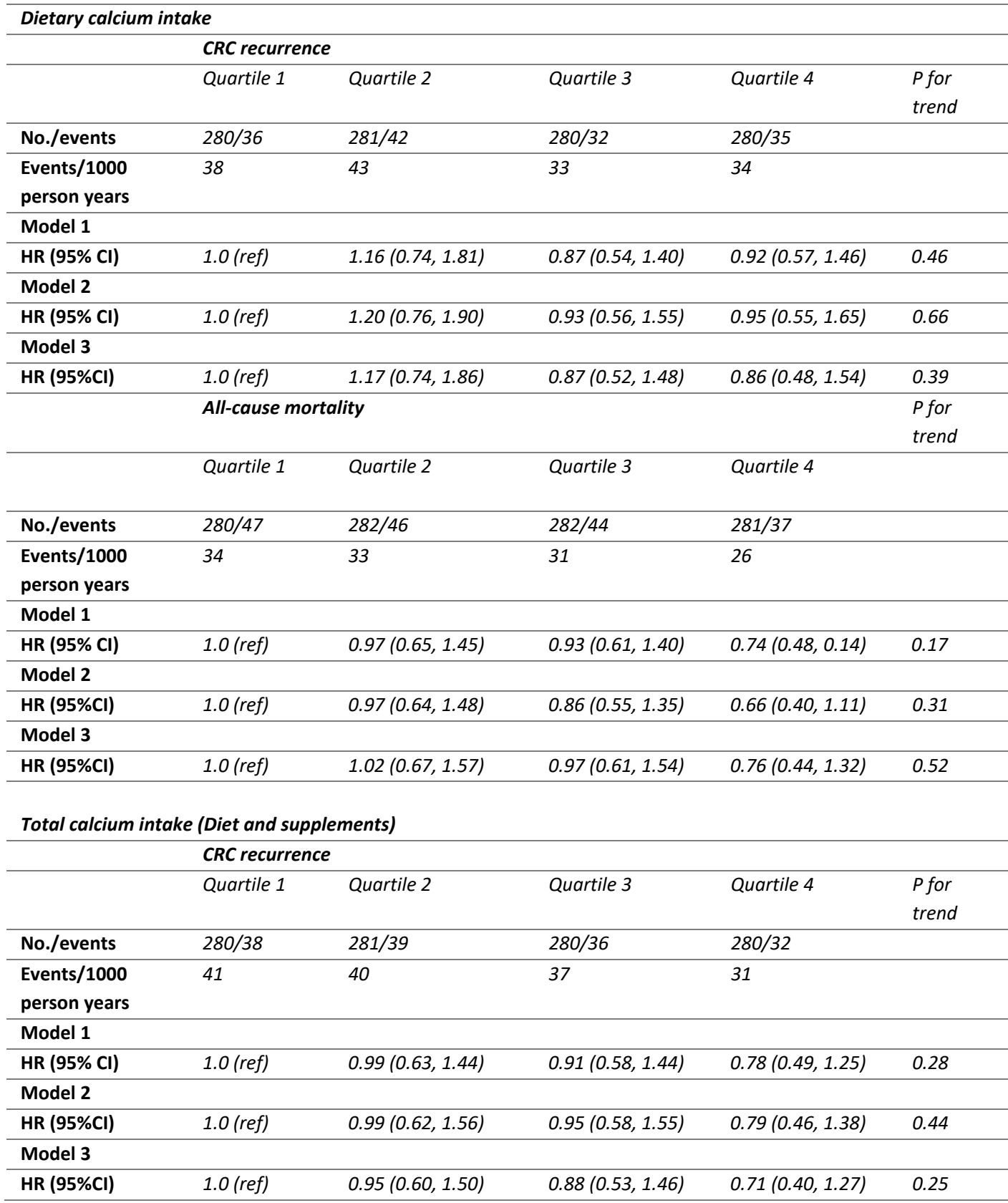




\begin{tabular}{|c|c|c|c|c|c|}
\hline & \multicolumn{5}{|c|}{ All-cause mortality } \\
\hline & Quartile 1 & Quartile 2 & Quartile 3 & Quartile 4 & $\begin{array}{l}\text { P for } \\
\text { trend }\end{array}$ \\
\hline No./events & $280 / 47$ & $282 / 46$ & $282 / 44$ & $281 / 37$ & \\
\hline $\begin{array}{l}\text { Events/1000 } \\
\text { person years }\end{array}$ & 36 & 31 & 36 & 23 & \\
\hline \multicolumn{6}{|l|}{ Model 1} \\
\hline $\mathrm{HR}(95 \% \mathrm{Cl})$ & 1.0 (ref) & $0.86(0.57,1.30)$ & $0.99(0.66,1.47)$ & $0.63(0.40,0.98)$ & 0.09 \\
\hline \multicolumn{6}{|l|}{ Model 2} \\
\hline HR $(95 \% \mathrm{Cl})$ & 1.0 (ref) & $0.86(0.57,1.32)$ & $0.88(0.57,1.36)$ & $0.58(0.34,0.98)$ & 0.07 \\
\hline \multicolumn{6}{|l|}{ Model 3} \\
\hline HR (95\%CI) & 1.0 (ref) & $0.97(0.63,1.49)$ & $1.06(0.66,1.67)$ & $0.70(0.40,1.21)$ & 0.27 \\
\hline
\end{tabular}

Quartiles of intake were cohort specific:

Dietary intake of calcium: COLON quartile 1 < 642 g/day; quartile $2642-855$ g/day; quartile 3 856-1088 g/day; quartile 4 > 1088 g/day. EnCoRe quartile 1 < 656 g/day; quartile 2 656-875 g/day; quartile 3 876-1144 g/day; quartile $4>1144 \mathrm{~g} /$ day

Total intake of calcium: COLON quartile 1 < 669 g/day; quartile $2669-888$ g/day; quartile $3889-1137$ g/day; quartile 4 > 1137 g/day. EnCoRe quartile 1 < 673 g/day; quartile $2673-930$ g/day; quartile 3 930-1230 g/day; quartile $4>1230 \mathrm{~g} /$ day

Model 1: crude Cox proportional hazard model.

Model 2: adjusted for age, sex, stage, BMI, physical activity (moderate to vigorous in hours/week), tumour location, cohort and total energy intake.

Model 3: as model 2 and additionally adjusted for dietary magnesium and $25(\mathrm{OH}) \mathrm{D}_{3}$ concentrations for the dietary intake models and total magnesium and $25(\mathrm{OH}) \mathrm{D}_{3}$ concentrations for total intake (diet and supplements) models.

$P$ values for trend were calculated by including the quartiles as a continuous variable in the model.

\section{THE INTERACTION BETWEEN 25(OH) $D_{3}$ CONCENTRATIONS AND MAGNESIUM OR CALCIUM INTAKE IN RELATION TO CRC RECURRENCE AND ALL-CAUSE MORTALITY}

\section{$\underline{\text { Vitamin D and magnesium }}$}

For $\mathrm{CRC}$ recurrence, no interaction between $25(\mathrm{OH}) \mathrm{D}_{3}$ concentrations and magnesium intake was observed (Table 5). In contrast, the risk of all-cause mortality was lowest in patients who had sufficient concentrations of $25(\mathrm{OH}) \mathrm{D}_{3}(\geq 50 \mathrm{nmol} / \mathrm{L})$ and a high magnesium intake ( $\geq 322 \mathrm{mg} /$ day for COLON and $\geq 383 \mathrm{mg} /$ day for EnCoRe) (HR 0.53, $95 \% \mathrm{Cl} 0.31,0.89)$ compared to patients with deficient $25(\mathrm{OH}) \mathrm{D}_{3}$ concentrations and a low magnesium intake. Borderline statistically significant multiplicative $(p=0.06)$ and additive (RERI $0.27,95 \% \mathrm{Cl}-0.08,0.61$ ) interactions were observed.

When analyzing the association for magnesium in strata of vitamin D status, the association between magnesium and all-cause mortality was statistically significant in patients who had sufficient vitamin D concentrations (HR $0.43,95 \% \mathrm{Cl} 0.25,0.76$ ), while no 
association was observed in patients who had deficient vitamin D concentrations (HR 0.94, $95 \% \mathrm{Cl} 0.52,1.69)$. When analyzing the association for vitamin $\mathrm{D}$ in strata of magnesium intake, the association between vitamin $\mathrm{D}$ concentrations and all-cause mortality was stronger in patients with a high magnesium intake (HR $0.69,95 \% \mathrm{Cl} 0.42,1.18)$ compared to patients with a low magnesium intake ( $\mathrm{HR} 0.98,95 \% \mathrm{Cl} 0.65,1.49)$, but observed associations were not statistically significant.

$\underline{\text { Vitamin D and calcium }}$

No interactions between calcium and vitamin $D$ with respect to $C R C$ recurrence and allcause mortality were observed (Table 6).

Similar results were observed when excluding patients who donated blood after the start of treatment (data not shown). 


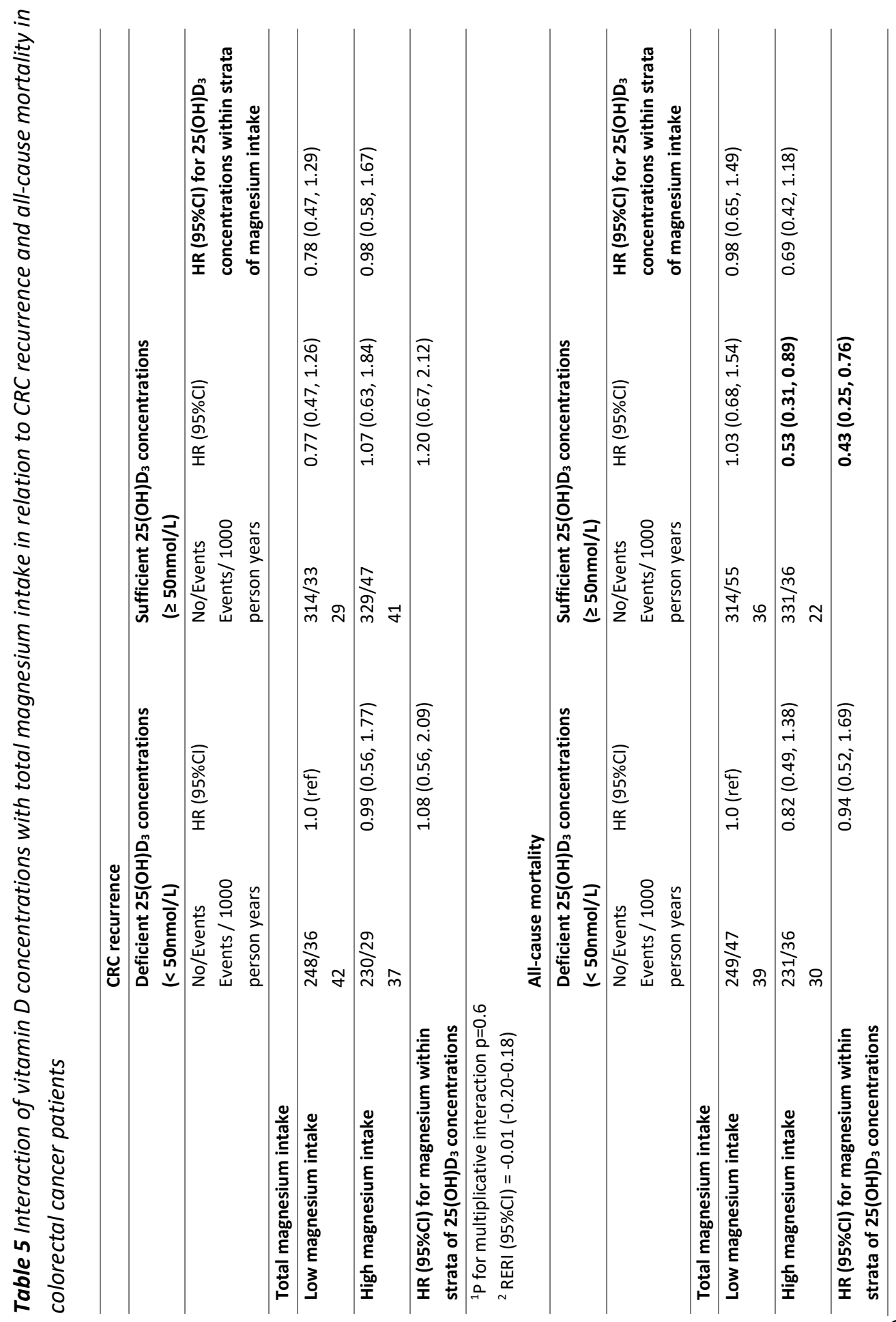




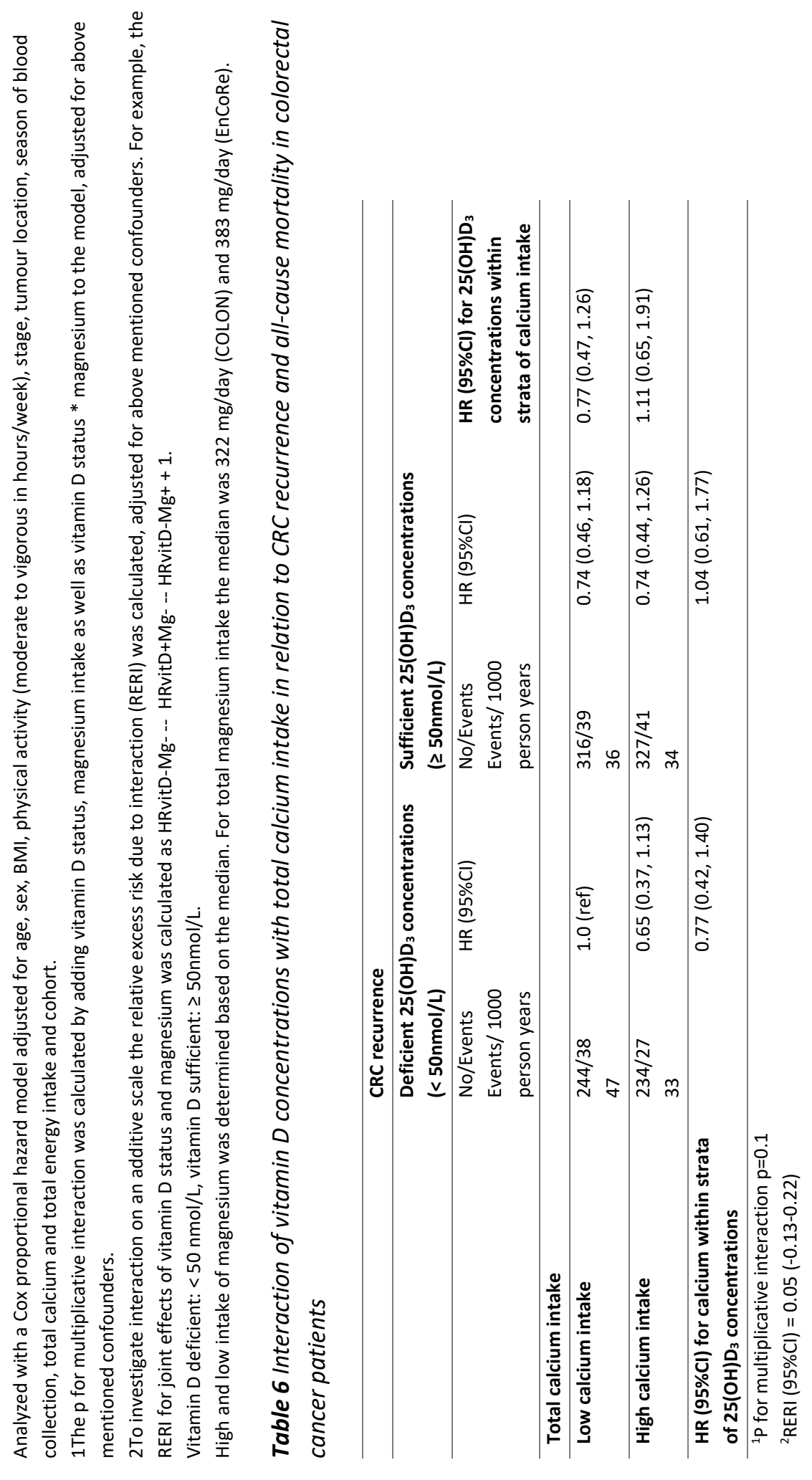




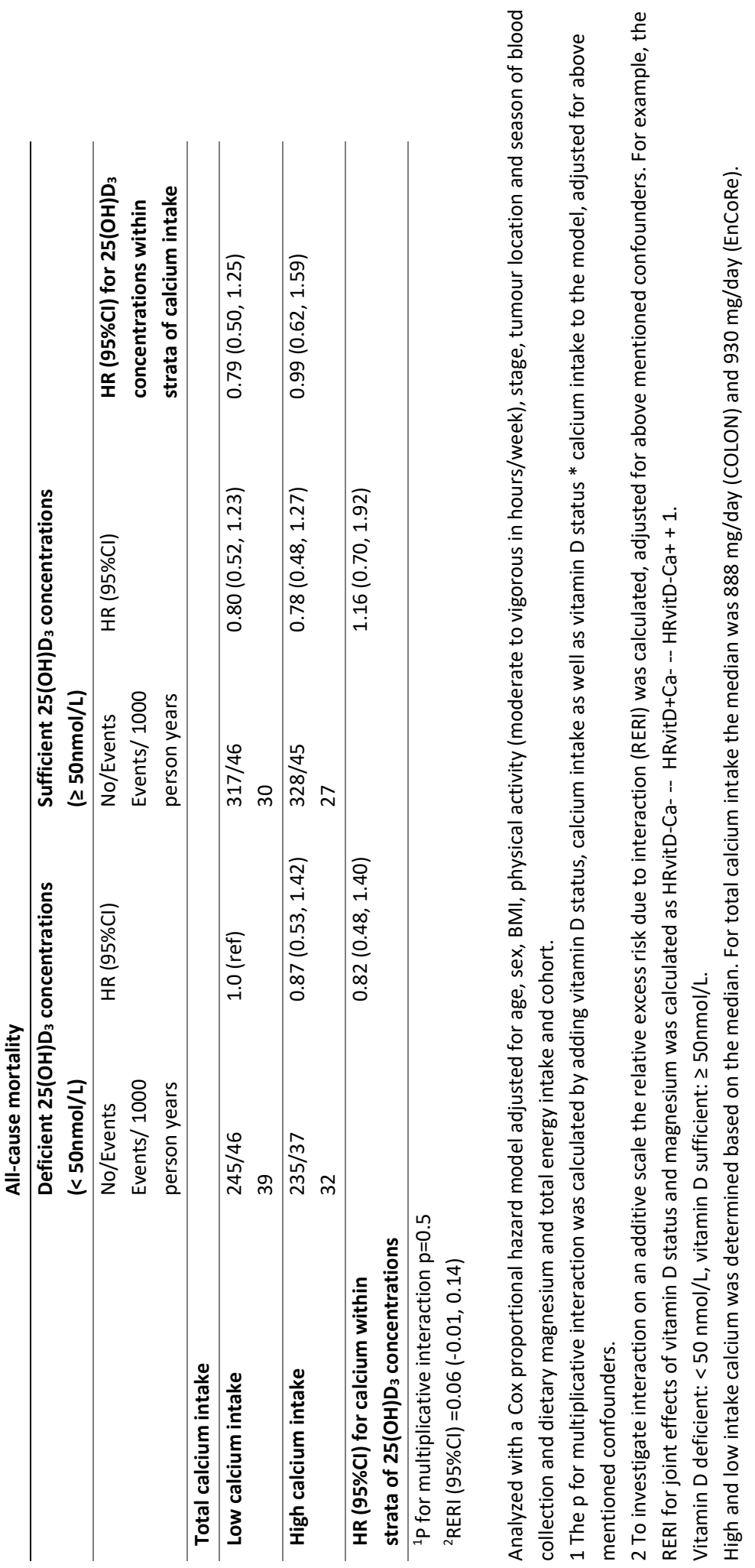




\section{DISCUSSION}

No associations between serum $25(\mathrm{OH}) \mathrm{D}_{3}$ concentrations and magnesium or calcium intake and CRC recurrence were observed in the current study. Lower vitamin D concentrations appear to be associated with a higher risk of all-cause mortality. An inverse association between magnesium intake, but not calcium intake, and all-cause mortality was observed. All-cause mortality was lowest in patients with sufficient vitamin D concentrations in combination with a high magnesium intake.

Severe vitamin D deficiency compared to sufficient vitamin D concentrations was statistically non-significant associated with a higher risk of all-cause mortality in our study. A recent meta-analysis including 11 studies among 7,718 CRC patients, observed a similar, but statistically significant association between $25(\mathrm{OH}) \mathrm{D}$ concentrations and all-cause mortality [12]. However, previous studies did not take magnesium intake into account, while we found an attenuated association after correction for magnesium intake. Magnesium is essential in the conversion of $25(\mathrm{OH}) \mathrm{D}_{3}$ to the active form of vitamin $\mathrm{D}$, $1,25(\mathrm{OH}) \mathrm{D}_{3}[15]$ and could potentially strengthen the association between vitamin $\mathrm{D}$ and outcomes.

In the present study, we observed a statistically significant lower risk of all-cause mortality for quartile 3 of magnesium intake ( $\approx 300-400 \mathrm{mg} /$ day), but not for quartile $4(\approx$ $>400 \mathrm{mg} /$ day) compared to quartile 1 ( $\approx<250 \mathrm{mg} /$ day). This association has not been reported before in CRC patients. In the general population, a dose-response meta-analysis showed an inverse non-linear association between dietary magnesium intake and the risk of all-cause mortality [36]. However, in this meta-analysis results were not adjusted for vitamin D concentrations, while results of our study showed that this is important. Furthermore, a possible explanation for the observation that we found a lower risk of allcause mortality for quartile 3 of magnesium intake, but not for quartile 4 is the interaction between magnesium and vitamin D. Findings of a recent randomized controlled trial with magnesium supplementation indicate that excessive magnesium intake $>400 \mathrm{mg} /$ day may actually reduce $25(\mathrm{OH}) \mathrm{D}_{3}$ concentrations [37]. Although increasing dietary magnesium intake until an optimum of approximately $400 \mathrm{mg} /$ day appears to reduce all-cause mortality, the HRs for the association between magnesium intake and recurrence were above 1 . However, confidence intervals were wide and no trend over quartiles of intake was observed. Thus, although a high magnesium intake seems beneficial in relation to allcause mortality, this may not be true with respect to recurrence.

A high calcium intake at diagnosis was inversely associated with all-cause mortality in CRC patients, however, after correcting for magnesium the HR for the association between calcium and all-cause mortality attenuated from 0.58 to 0.70 . Results of previous studies $[20,21]$ suggest an inverse association between high post-diagnostic calcium intake and all-cause mortality in CRC survivors. However, these previous studies did not correct for 
magnesium intake. Although it seems that the association between calcium and mortality is partly caused by magnesium, it should be noted that magnesium and calcium intake are correlated $(r=0.6)$ thus the effect of magnesium and calcium can probably not be disentangled completely. Of note, albeit not statistically significant, a high calcium intake appears to be associated with a lower risk of recurrence as well. Therefore, based on our data, we can carefully conclude that calcium is at least not harmful for CRC patients.

The lowest risk of all-cause mortality was found in patients who had both high vitamin $D$ concentrations and a high magnesium intake. In addition, the association between vitamin D concentrations and all-cause mortality is only present in those with a high magnesium intake. This is in line with previous research in the general population, showing a stronger association between vitamin $D$ concentrations and all-cause mortality in those with a higher magnesium intake [15]. Thus, if the observed associations are causal, the presence of an adequate status of both nutrients is essential in lowering the risk of all-cause mortality. Considering the importance of magnesium for the enzymatic conversion of vitamin $D$ into its active form $[15,37]$, magnesium might be crucial in maintaining a sufficient vitamin $D$ status $[15,16,37]$. The active form of vitamin $D$ is hypothesized to have beneficial effects on cancer prognosis [38]. There are also indications that vitamin $D$ influences CRC mortality by modulation of immune and inflammatory responses [39]. In addition, magnesium deficiency is associated with chronic low grade inflammation [40]. Since vitamin $D$ and magnesium are both suggested to influence systemic inflammation $[40,41]$, it is tempting to speculate that vitamin $D$ and magnesium contribute to a lowered inflammatory status via shared mechanisms, possible resulting in better survival rates.

In our study among stage I-III patients, no associations between $25(\mathrm{OH}) \mathrm{D}_{3}$, magnesium or calcium and CRC recurrence were observed. One previous study investigating the association between $25(\mathrm{OH}) \mathrm{D}_{3}$ concentrations and $\mathrm{CRC}$ recurrence reported a strong inverse association (HR $0.3795 \% \mathrm{Cl} 0.18,0.84$ )[8]. However, this study population consisted of CRC patients with liver metastasis (stage IV), which is a very specific population at high risk for recurrences. Although we did not observe an association between $25(\mathrm{OH}) \mathrm{D}_{3}$ concentrations, magnesium and calcium intake and $\mathrm{CRC}$ recurrence, this should be further investigated before a solid conclusion can be drawn.

Since, the presence of an adequate status of both magnesium and vitamin $D$ seems to be essential in lowering the risk of all-cause mortality, attention should be paid to both vitamin $D$ concentrations as well as magnesium intake. However, more data is needed to draw firm conclusions and provide practical guidance. At first, the underlying mechanisms explaining the interaction between magnesium and vitamin $D$ in attenuating all-cause mortality should be further unraveled. Second, diet and lifestyle intervention studies should investigate whether an increase in magnesium intake and vitamin D concentrations results in better CRC prognosis. In these studies, also the influence of different sources of 
magnesium (diet or supplements) and vitamin D (sunlight exposure or diet and supplements) should be investigated.

The present study had some limitations. First, we did not analyze concentrations of magnesium and calcium. Blood concentrations of these minerals are tightly regulated and $<1 \%$ of the total body magnesium and calcium is circulating $[42,43]$, thus measuring magnesium and calcium blood concentrations would not likely have resulted in more information [16]. Second, the number of events was relatively low in our study population ( $n=155$ for recurrence; $n=191$ for mortality), which limits the power to detect statistically significant associations, especially in the interaction analyses. Nonetheless, a significant interaction between magnesium and vitamin $\mathrm{D}$ was observed for all-cause mortality. Third, it could be that participants of our study are relatively health conscious, which will probably lead to an attenuation of the real effect. Furthermore, we had no data available about the cause of death. Therefore, we were not able to perform analyses with diseasespecific mortality as an outcome. Finally, results of this study can only be generalized to the western population. The present study also had some important strengths. First, to the best of our knowledge this study was the first to investigate $25(\mathrm{OH}) \mathrm{D}_{3}$ concentrations, magnesium and calcium intake, individually and jointly, in relation to CRC recurrence and all-cause mortality. Second, we could investigate the influence of total magnesium and calcium intake, as we obtained information about dietary as well as supplemental intake. Finally, due the availability of detailed data on diet and other clinical and lifestyle factors, we could adjust for the most relevant confounders, although residual confounding can never be fully excluded.

To conclude, we observed that $25(\mathrm{OH}) \mathrm{D}_{3}$ and magnesium may work synergistically in decreasing the risk of all-cause mortality in colorectal cancer patients. Although our results should be confirmed in diet and lifestyle intervention studies, our findings could contribute to improving recommendations regarding magnesium and vitamin $D$ intake for newly diagnosed colorectal cancer patients. 


\section{ADDITIONAL INFORMATION}

\section{FUNDING}

The COLON study was financially supported by Wereld Kanker Onderzoek Fonds (WKOF) \& World Cancer Research Fund International (WCRF International) as well as by funds from grant 2014/1179 as part of the World Cancer Research Fund International Regular Grant Programme; Alpe d'Huzes/Dutch Cancer Society (UM 2012-5653, UW 2013-5927, UW 2015-7946); and ERA-NET on Translational Cancer Research (TRANSCAN:Dutch Cancer Society (UW2013-6397, UW2014-6877) and the Netherlands Organization for Health Research and Development (ZonMw, the Netherlands). The EnCoRe study was supported by grants from the Stichting Alpe d'HuZes within the research program 'Leven met kanker' of the Dutch Cancer Society (Grant No. UM2010-4867 and UM 2012-5653) and by a grant from Kankeronderzoekfonds Limburg as part of Health Foundation Limburg (Grant No. 00005739).

\section{AUTHORS' CONTRIBUTION}

E Wesselink, DE Kok, MJL Bours, HJW de Wilt, RF Witkamp, MP Weijenberg, E Kampman and FJB van Duijnhoven contributed to the design and the conceptualization of this study. E Wesselink, HJW de Wilt, H van Baar, AJMR Geijsen, B Hansson, ETP Keulen, J van den Ouweland and $M$ van Zutphen contributed to recruitment of participants and the data collection. Statistical data analyses were done by E Wesselink. The manuscript was drafted by $E$ Wesselink and FJB van Duijnhoven, and all authors critically read and revised the manuscript. All authors approved the final version of the manuscript.

\section{ACKNOWLEDGEMENT}

The authors would like to thank the participants of the COLON study and the investigators at Wageningen University \& Research and the co-workers from the following hospitals for their involvement in recruitment for the COLON study: Hospital Gelderse Vallei, Ede; Radboudumc, Nijmegen; Slingeland Hospital, Doetinchem; Canisius Wilhelmina Hospital, Nijmegen; Rijnstate Hospital, Arnhem; Gelre Hospitals, Apeldoorn/Zutphen; Hospital Bernhoven, Uden; Isala, Zwolle; ZGT, Almelo; Martini Hospital, Groningen; Admiraal de Ruyter Hospital, Goes/Vlissingen. We would like to thank all participants of the EnCoRe study and the health professionals in the three hospitals involved in the recruitment of participants of the study: Maastricht University Medical Center, VieCuri Medical Center, and Zuyderland Medical Center. We would also like to thank the MEMIC center for data and information management for facilitating the logistic processes and data management of our study. Furthermore, we would like to thank the research dieticians and research assistant who are responsible for patient inclusion and follow-up, performing home visits, as well as data collection and processing. 


\section{DATA SHARING}

Since the data consist of identifying cohort information, some access restrictions apply and therefore cannot be made publicly available. Data will be shared with permission from the acting committee of the COLON Study. Requests for data can be sent to Dr. Fränzel van Duijnhoven, Division of Human Nutrition and Health, Wageningen University \& Research, The Netherlands.

Email: franzel.vanduijnhoven@wur.nl. 


\section{REFERENCES}

[1] Ng K, Meyerhardt JA, Wu K, Feskanich D, Hollis BW, Giovannucci EL, et al. Circulating 25-hydroxyvitamin d levels and survival in patients with colorectal cancer. Journal of Clinical Oncology. 2008;26:2984-91.

[2] Mezawa H, Sugiura T, Watanabe M, Norizoe C, Takahashi D, Shimojima A, et al. Serum vitamin D levels and survival of patients with colorectal cancer: post-hoc analysis of a prospective cohort study. BMC Cancer. 2010;10:347.

[3] Ng K, Sargent DJ, Goldberg RM, Meyerhardt JA, Green EM, Pitot HC, et al. Vitamin D status in patients with stage IV colorectal cancer: findings from Intergroup trial N9741. Journal of Clinical Oncology. 2011;29:1599-606. [4] Fedirko V, Riboli E, Tjonneland A, Ferrari P, Olsen A, Bueno-de-Mesquita HB, et al. Prediagnostic 25hydroxyvitamin D, VDR and CASR polymorphisms, and survival in patients with colorectal cancer in western European ppulations. Cancer epidemiology, biomarkers \& prevention : a publication of the American Association for Cancer Research, cosponsored by the American Society of Preventive Oncology. 2012;21:582-93.

[5] Tretli S, Schwartz GG, Torjesen PA, Robsahm TE. Serum levels of 25-hydroxyvitamin D and survival in Norwegian patients with cancer of breast, colon, lung, and lymphoma: a population-based study. Cancer Causes Control. 2012;23:363-70.

[6] Zgaga L, Theodoratou E, Farrington SM, Din FV, Ooi LY, Glodzik D, et al. Plasma Vitamin D Concentration Influences Survival Outcome After a Diagnosis of Colorectal Cancer. Journal of Clinical Oncology. 2014;32:2430-9. [7] Wesa KM, Segal NH, Cronin AM, Sjoberg DD, Jacobs GN, Coleton MI, et al. Serum 25-hydroxy vitamin D and survival in advanced colorectal cancer: a retrospective analysis. Nutrition and cancer. 2015;67:424-30.

[8] Facciorusso A, Del Prete V, Muscatiello N, Crucinio N, Barone M. Prognostic role of 25-hydroxyvitamin D in patients with liver metastases from colorectal cancer treated with radiofrequency ablation. Journal of gastroenterology and hepatology. 2016;31:1483-8.

[9] Yang L, Chen H, Zhao M, Peng P. Prognostic value of circulating vitamin D binding protein, total, free and bioavailable 25-hydroxy vitamin D in patients with colorectal cancer. Oncotarget. 2017;8:40214-21.

[10] Maalmi H, Walter V, Jansen L, Chang-Claude J, Owen RW, Ulrich A, et al. Relationship of very low serum 25hydroxyvitamin D3 levels with long-term survival in a large cohort of colorectal cancer patients from Germany. European journal of epidemiology. 2017;32:961-71.

[11] Cooney RV, Chai W, Franke AA, Wilkens LR, Kolonel LN, Le Marchand L. C-reactive protein, lipid-soluble micronutrients, and survival in colorectal cancer patients. Cancer Epidemiology, Biomarkers \& Prevention. 2013;22:1278-88.

[12] Maalmi H, Walter V, Jansen L, Boakye D, Schöttker B, Hoffmeister M, et al. Association between Blood 25Hydroxyvitamin D Levels and Survival in Colorectal Cancer Patients: An Updated Systematic Review and MetaAnalysis. Nutrients. 2018;10:896.

[13] Custers JA, Gielissen MF, Janssen SH, de Wilt JH, Prins JB. Fear of cancer recurrence in colorectal cancer survivors. Supportive Care in Cancer. 2016;24:555-62.

[14] Elferink MAG, de Jong KP, Klaase JM, Siemerink EJ, de Wilt JHW. Metachronous metastases from colorectal cancer: a population-based study in North-East Netherlands. International journal of colorectal disease.

2015;30:205-12.

[15] Deng X, Song Y, Manson JE, Signorello LB, Zhang SM, Shrubsole MJ, et al. Magnesium, vitamin D status and mortality: results from US National Health and Nutrition Examination Survey (NHANES) 2001 to 2006 and NHANES III. BMC medicine. 2013;11:187.

[16] Uwitonze AM, Razzaque MS. Role of magnesium in vitamin D activation and function. The Journal of the American Osteopathic Association 2018;118:181-9.

[17] Rosanoff A, Dai Q, Shapses SA. Essential Nutrient Interactions: Does Low or Suboptimal Magnesium Status Interact with Vitamin D and/or Calcium Status? Advances in nutrition (Bethesda, Md). 2016;7:25-43.

[18] Reddy V, Sivakumar B. Magnesium-dependent vitamin-D-resistant rickets. The Lancet. 1974;303:963-5.

[19] Lips P. Interaction between vitamin D and calcium. Scandinavian Journal of Clinical and Laboratory Investigation. 2012;72:60-4. 
[20] Yang B, McCullough ML, Gapstur SM, Jacobs EJ, Bostick RM, Fedirko V, et al. Calcium, vitamin D, dairy products, and mortality among colorectal cancer survivors: the Cancer Prevention Study-II Nutrition Cohort. Journal of Clinical Oncology. 2014;32:2335-43.

[21] Yang W, Ma Y, Smith-Warner S, Song M, Wu K, Wang M, et al. Calcium Intake and Survival after Colorectal Cancer Diagnosis. Clinical Cancer Research. 2019;25:1980-8.

[22] Shaukat A, Scouras N, Schünemann HJ. Role of supplemental calcium in the recurrence of colorectal adenomas: a metaanalysis of randomized controlled trials. The American journal of gastroenterology. 2005;100:390.

[23] Baron JA, Barry EL, Mott LA, Rees JR, Sandler RS, Snover DC, et al. A Trial of Calcium and Vitamin D for the Prevention of Colorectal Adenomas. The New England journal of medicine. 2015;373:1519-30.

[24] Crockett SD, Barry EL, Mott LA, Ahnen DJ, Robertson DJ, Anderson JC, et al. Calcium and vitamin D supplementation and increased risk of serrated polyps: results from a randomised clinical trial. Gut. 2018;68:47586.

[25] Winkels RM, Heine-Broring RC, van Zutphen M, van Harten-Gerritsen S, Kok DE, van Duijnhoven FJ, et al. The COLON study: Colorectal cancer: Longitudinal, Observational study on Nutritional and lifestyle factors that may influence colorectal tumour recurrence, survival and quality of life. BMC Cancer. 2014;14:374.

[26] van Roekel EH, Bours MJ, de Brouwer CP, Ten Napel H, Sanduleanu S, Beets GL, et al. The Applicability of the International Classification of Functioning, Disability, and Health to Study Lifestyle and Quality of Life of Colorectal Cancer Survivors. Cancer epidemiology, biomarkers \& prevention : a publication of the American Association for Cancer Research, cosponsored by the American Society of Preventive Oncology. 2014;23:1394405.

[27] van den Ouweland JM, Beijers AM, van Daal H. Overestimation of 25-hydroxyvitamin D3 by increased ionisation efficiency of 3-epi-25-hydroxyvitamin D3 in LC-MS/MS methods not separating both metabolites as determined by an LC-MS/MS method for separate quantification of 25-hydroxyvitamin D3, 3-epi-25hydroxyvitamin D3 and 25-hydroxyvitamin D2 in human serum. Journal of Chromatography B. 2014;967:195-202. [28] Aranow C. Vitamin D and the immune system. Journal of investigative medicine. 2011;59:881-6.

[29] Koole JL, Bours MJL, Breedveld-Peters JJL, van Roekel EH, van Dongen MCJM, Eussen SJPM, et al. Evaluating the Validity of a Food Frequency Questionnaire in Comparison with a 7-Day Dietary Record for Measuring Dietary Intake in a Population of Survivors of Colorectal Cancer. Journal of the Academy of Nutrition and Dietetics. 2020;120:245-57.

[30] Dutch Nutrition Center. NEVO Nederlandse Voedingsmiddelen tabel 2011 (In Englisch: Dutch Food Composition Table).

[31] Wendel-Vos GC, Schuit AJ, Saris WH, Kromhout D. Reproducibility and relative validity of the short questionnaire to assess health-enhancing physical activity. Journal of clinical epidemiology. 2003;56:1163-9.

[32] Van Leersum N, Snijders H, Henneman D, Kolfschoten N, Gooiker G, Ten Berge M, et al. The Dutch surgical colorectal audit. European Journal of Surgical Oncology (EJSO). 2013;39:1063-70.

[33] Holick MF. Vitamin D deficiency. New England Journal of Medicine. 2007;357:266-81.

[34] Knol MJ, VanderWeele TJ. Recommendations for presenting analyses of effect modification and interaction. International journal of epidemiology. 2012;41:514-20.

[35] Knol MJ, VanderWeele TJ, Groenwold RH, Klungel OH, Rovers MM, Grobbee DE. Estimating measures of interaction on an additive scale for preventive exposures. European journal of epidemiology. 2011;26:433-8. [36] Fang X, Wang K, Han D, He X, Wei J, Zhao L, et al. Dietary magnesium intake and the risk of cardiovascular disease, type 2 diabetes, and all-cause mortality: a dose-response meta-analysis of prospective cohort studies. BMC medicine. 2016;14:210.

[37] Dai Q, Zhu X, Manson JE, Song Y, Li X, Franke AA, et al. Magnesium status and supplementation influence vitamin $D$ status and metabolism: results from a randomized trial. The American journal of clinical nutrition. 2018;108:1249-58.

[38] Ferrer-Mayorga G, Larriba MJ, Crespo P, Munoz A. Mechanisms of action of vitamin D in colon cancer. The Journal of steroid biochemistry and molecular biology. 2018;185:1-6. 
[39] Krishnan AV, Feldman D. Mechanisms of the anti-cancer and anti-inflammatory actions of vitamin D. Annual review of pharmacology and toxicology. 2011;51:311-36.

[40] Nielsen FH. Magnesium deficiency and increased inflammation: current perspectives. Journal of inflammation research. 2018;11:25.

[41] Krishnan AV, Feldman D. Mechanisms of the anti-cancer and anti-inflammatory actions of vitamin D. Annual review of pharmacology and toxicology. 2011;51:311-36.

[42] De Baaij JH, Hoenderop JG, Bindels RJ. Regulation of magnesium balance: lessons learned from human genetic disease. Clinical kidney journal. 2012;5:i15-i24.

[43] Peacock M. Calcium Metabolism in Health and Disease. Clinical Journal of the American Society of Nephrology. 2010;5:S23-S30. 



\title{
CHAPTER 4
}

\section{SUFFICIENT 25-HYDROXYVITAMIN D LEVELS TWO YEARS AFTER COLORECTAL CANCER DIAGNOSIS ARE ASSOCIATED WITH A LOWER RISK OF ALL-CAUSE MORTALITY.}

\author{
Evertine Wesselink \\ Dieuwertje E. Kok \\ Johannes H.W. de Wilt \\ Martijn J.L. Bours \\ Moniek van Zutphen \\ Eric T.P. Keulen \\ Flip M. Kruyt \\ Stephanie O. Breukink \\ Ewout A. Kouwenhoven \\ Jody van den Ouweland \\ Matty P. Weijenberg \\ Ellen Kampman
}

Franzel J.B. van Duijnhoven

Cancer Epidemiol Biomarkers and Prevention 2021 Apr;30(4):765-773. 


\section{ABSTRACT}

Background: Whether changes in vitamin $\mathrm{D}\left(25(\mathrm{OH}) \mathrm{D}_{3}\right)$ levels after colorectal cancer $(\mathrm{CRC})$ diagnosis influence clinical outcomes is unclear. We investigated associations of trajectories of $25(\mathrm{OH}) \mathrm{D} 3$ levels with recurrence and all-cause mortality.

Methods: In total, 679 patients were included in our data-analyses. Trajectories of $25(\mathrm{OH}) \mathrm{D}_{3}$ levels were defined on vitamin $\mathrm{D}$ status at diagnosis, and at six months and two years after diagnosis. Observed trajectories of $25(\mathrm{OH}) \mathrm{D}_{3}$ levels were consistent deficient levels (20\%), consistent sufficient levels (39\%), increasing levels (20\%) and a temporary drop in levels (13\%). Associations of trajectories of $25(\mathrm{OH}) \mathrm{D}_{3}$ with recurrence and all-cause mortality were assessed using multivariable Cox proportional hazard regression models.

Results: During a follow-up time of 2.2 years for recurrence and 3.5 years for all-cause mortality, 31 and 65 events occurred, respectively. No statistically significant associations were observed for vitamin $D$ trajectories and the risk of recurrence. Patients who were consistently sufficient compared to patients who were consistently deficient had a lower risk of all-cause mortality ( $\mathrm{HR} 0.3995 \% \mathrm{Cl} 0.21-0.73)$. The risk of all-cause mortality seems lower in patients with increasing levels or a temporary drop in levels (HR $0.5495 \% \mathrm{Cl} 0.27$ 1.10 and $\mathrm{HR} 0.4095 \% \mathrm{Cl} 0.17-0.93$ ) relative to patients with consistent deficient levels.

Conclusions: CRC patients following a trajectory characterized by sufficient levels of $25(\mathrm{OH}) \mathrm{D}_{3}$ two years after diagnosis all appeared to have a lower risk of all-cause mortality compared to patients having consistent deficient levels.

Impact: Further studies should investigate how trajectories of $25(\mathrm{OH}) \mathrm{D}_{3}$ levels are associated with $\mathrm{CRC}$ recurrence.

Keywords: vitamin D, 25(OH) $\mathrm{D}_{3}$ levels, colorectal cancer, all-cause mortality, recurrence. 


\section{INTRODUCTION}

Evidence is accumulating that twenty-five hydroxy vitamin $\mathrm{D}_{3}\left(25(\mathrm{OH}) \mathrm{D}_{3}\right)$ levels are inversely associated with all-cause as well as colorectal cancer (CRC)-specific mortality in CRC patients [1-12]. Maalmi and colleagues published a meta-analysis, including 11 original studies with a total of 7,718 CRC patients [13]. Pooled effect estimates comparing the highest versus lowest category of circulating $25(\mathrm{OH}) \mathrm{D}_{3}$ at diagnosis showed a hazard ratio (HR) of 0.68 (95\% confidence interval $(\mathrm{Cl}) 0.55-0.85)$ for all-cause mortality [13]. In addition to mortality, a study including 2,910 patients diagnosed with CRC observed a $32 \%$ higher risk of recurrence in patients with very low plasma $25(\mathrm{OH}) \mathrm{D}_{3}$ concentrations at diagnosis ( $<11.8 \mathrm{nmol} / \mathrm{L}$ ) compared to patients with $25(\mathrm{OH}) \mathrm{D}_{3}$ levels $>45.2 \mathrm{nmol} / \mathrm{L}$ [10]. On the contrary, in our previous work, no association between $25(\mathrm{OH}) \mathrm{D}_{3}$ levels at CRC diagnosis and CRC recurrence was observed when comparing patients with severe deficient levels (<30 $\mathrm{nmol} / \mathrm{L}$ ) with patients with sufficient levels $(50-75 \mathrm{nmol} / \mathrm{L})$ [14]. However, in the afore mentioned studies $25(\mathrm{OH}) \mathrm{D}_{3}$ was assessed only once, often at time of diagnosis.

Vitamin D deficiency ( $<50 \mathrm{nmol} / \mathrm{L}$ ) is highly prevalent at diagnosis $(40-80 \%)$ in CRC patients $[13,15]$. Cancer treatment may further challenge achievement and maintenance of sufficient ( $>50 \mathrm{nmol} / \mathrm{L}) 25(\mathrm{OH}) \mathrm{D}_{3}$ levels for CRC patients $[15,16]$. As the main source of vitamin $D$ is sunlight, other factors such as spending less time outdoors due to being bedbound or experiencing fatigue may also play a role in a decrease of $25(\mathrm{OH}) \mathrm{D}_{3}$ levels after diagnosis $[15,16]$. On the other hand, $25(\mathrm{OH}) \mathrm{D}_{3}$ levels can be improved by an increased exposure to sunlight and by taking vitamin D supplementation $[15,16]$.

Research focusing on changes in $25(\mathrm{OH}) \mathrm{D}_{3}$ levels after diagnosis and associations with CRC outcomes remains scarce. One previous study combining data of in total 3,535 CRC patients observed a post-operative fall in $25(\mathrm{OH}) \mathrm{D}_{3}$ levels followed by a recovery [12]. In this study pre-operative $25(\mathrm{OH}) \mathrm{D}_{3}$ levels were available for 486 patients, while postoperative $25(\mathrm{OH}) \mathrm{D}_{3}$ levels were assessed in 3,049 other patients [12]. Higher versus lower pre-operative as well as higher versus lower post-operative $25(\mathrm{OH}) \mathrm{D}_{3}$ levels were associated with a lower risk of all-cause mortality (HR $0.3095 \% \mathrm{Cl} 0.12-0.71$; HR 0.65 $95 \% \mathrm{Cl} 0.51-0.81$, respectively) [12]. Although timing of $25(\mathrm{OH}) \mathrm{D}_{3}$ levels (pre versus postoperative) was investigated in this study, one measurement per patients was taken, while $25(\mathrm{OH}) \mathrm{D}_{3}$ levels are likely to change over time. Those changes over time, for example a steep decrease during cancer treatment or delayed recovery of $25(\mathrm{OH}) \mathrm{D}_{3}$ levels after treatment, could also affect health outcomes in CRC patients. Hence, investigating trajectories of $25(\mathrm{OH}) \mathrm{D}_{3}$ levels over time is of importance. Therefore, the aim of the present study was to investigate our hypothesis that trajectories of $25(\mathrm{OH}) \mathrm{D}_{3}$ levels over time are associated with $\mathrm{CRC}$ recurrence and all-cause mortality. 


\section{METHODS}

\section{STUDY DESIGN}

Data from two prospective cohort studies was used, the COLON study and the EnCoRe study. The original aim of the COLON study was to assess associations of diet and other lifestyle factors, with CRC recurrence, survival and quality of life. The original aim of the EnCoRe study was to assess associations of diet and other lifestyle factors with quality of life in CRC patients. The designs of the COLON study [17] (ClinicalTrials.gov identifier: NCT03191110) and the EnCoRe study [18] (trialregister.nl identifier: NL6904) have been described previously. In short, in both studies, newly diagnosed CRC patients were recruited directly after diagnosis in 14 hospitals and were followed during and after treatment. Men and women above the age of 18 were eligible. In the COLON study, patients with a first primary stage I-IV CRC were eligible. In the EnCoRe study, patients with primary stage I-III CRC were eligible. The COLON and EnCoRe studies were was approved by regional Medical Ethics Committees (NL30446.091.09 and NL38786.068.11, respectively). All patients signed informed consent.

\section{BLOOD COLLECTION AND VITAMIN D LEVELS}

For the COLON study, blood samples were obtained in the hospital shortly after diagnosis, six months and two year after diagnosis. For the EnCoRe study, blood samples shortly after diagnosis were obtained in the hospital or by a research assistant during a home visit before start of treatment and at six weeks, six months, one year and two years after the end of treatment. To harmonize the data of both cohorts, we selected the time point in the EnCoRe cohort closest to either six months (median 5.8 IQR 5.4-6.5 months) or two years (median 23.9 IQR 23.4-24.7 months) after diagnosis as the second and third time points for these analyses. For both studies, blood samples were obtained in a serum tube, centrifuged and serum was aliquoted and stored in a freezer at $-80^{\circ} \mathrm{C}$ until further analysis.

For both cohorts, serum $25(\mathrm{OH}) \mathrm{D}_{3}$ levels, the most reliable measurement of an individual's vitamin D status [19], were measured by isotope-dilution liquid chromatography tandem mass spectrometry (LC-MS/MS) in the Canisius Wilhelmina Hospital, Nijmegen, the Netherlands [20]. The inter-assay coefficients of variation were $5.3 \%, 3.1 \%$ and $2.9 \%$ at $25(\mathrm{OH}) \mathrm{D}_{3}$ levels of 39.0, 92.5 and $127.0 \mathrm{nmol} / \mathrm{L}$, respectively.

To correct for the influence of season on vitamin D levels, $25(\mathrm{OH}) \mathrm{D}_{3}$ levels were adjusted for week of blood collection using the locally weighted polynomial regression (LOESS) method $[21,22]$. To obtain interpretable $25(\mathrm{OH}) \mathrm{D}_{3}$ level values, we added the mean $25(\mathrm{OH}) \mathrm{D}_{3}$ level to the residuals of the LOESS method. 


\section{VITAMIN D TRAJECTORIES}

Vitamin D deficiency was defined as $25(\mathrm{OH}) \mathrm{D}_{3}$ levels lower than $50 \mathrm{nmol} / \mathrm{L}$, while vitamin D sufficiency was defined as $25(\mathrm{OH}) \mathrm{D}_{3}$ levels of $50 \mathrm{nmol} / \mathrm{L}$ or higher [23]. Based on the season adjusted vitamin D status (deficient/sufficient) measured at the three time points, four main vitamin D trajectories were observed in the data. Patients who were deficient at all three time points were categorized into trajectory 1 (consistent deficient levels). Patients who were sufficient at all three time points were categorized into trajectory 2 (consistent sufficient levels). Patients who were deficient at diagnosis, but sufficient after two years were categorized into trajectory 3 (increasing levels). Patients who were sufficient at diagnosis, deficient after six months and again sufficient after two years were categorized into trajectory 4 (temporary drop in levels). Categorization into trajectories was done based on crude season-adjusted $25(\mathrm{OH}) \mathrm{D}_{3}$ levels.

\section{DATA COLLeCtion}

Habitual dietary intake in the month (COLON study) or year (EnCoRe study) preceding diagnosis was assessed using a semi-quantitative food frequency questionnaire. At the other time points, i.e. six months and two years after diagnosis, habitual dietary intake was assessed with a semi-quantitative food frequency questionnaire in the COLON study and a 7-day food diary in the EnCoRe study. The FFQ used in the COLON study consist of 204 items. The FFQ used in the EnCoRe study consists of 253 items and is recently validated for macro and micronutrients. Relative to the dietary record, the validity of the FFQ was moderate too good for most nutrients including magnesium ( $r=0.61$ ) and vitamin $D(r=0.67)$ [24]. Dietary intake of vitamin D, magnesium, calcium, alcohol and fibre was calculated for each food item based on frequency of intake, number of portions and portion size, as well as the type of product. Mean daily intakes were calculated by adding all items containing the respective nutrient using data from the 2011 Dutch food composition table [25]. For the COLON study, supplement use was assessed by a dietary supplement questionnaire [17]. The dietary supplement questionnaire contains questions on use of single or multivitamin/minerals supplements and on the dosage and frequency of intake. In the EnCoRe study, supplement use was assessed by a research dietician during a home visit, by collecting information on dosage, frequency, duration, and ingredients from supplement packages on standardized forms. Supplement use was in both cohorts defined as using supplements at least once a week for at least one month in the last year or since the previous measurement time point. Additionally, vitamins or minerals that were used once a month, but contained a high dose to cover the intake for a longer period of time (e.g. D-CURE 25.000 IE Cholecalciferol supplementation), were also classified as supplement use. Total intake of magnesium, calcium and vitamin D was calculated by summing intake from diet and intake from dietary supplements. 
Information on demographics and lifestyle was obtained using self-administered questionnaires at the same time as the blood samples were collected in both cohorts. Information on height and weight was collected using self-administered questionnaires in the COLON study. In the EnCoRe study, these measurements were performed by research dieticians during home visits. Physical activity in the past two months was assessed using the Short QUestionnaire to ASsess Health-enhancing physical activity (SQUASH) in both cohorts [26].

Clinical data, such as stage of disease, tumour location (colon/rectum), date of start of treatment, type of treatment (surgery, neo-adjuvant/adjuvant chemotherapy, radiotherapy) and presence of comorbidities at diagnosis for both cohorts were derived from the Dutch ColoRectal Audit [27].

\section{CRC OUTCOMES}

Information on recurrence, defined as a local regional recurrence or distant metastases, was collected from medical records by the Dutch Cancer Registration. Information on mortality was gathered from linkage with the Municipal Personal Record Database.

Follow-up time for recurrence was calculated starting from the date of blood collection at the two year time point until date of recurrence or until the date recurrence status was updated (COLON: February 2018; EnCoRe: March 2018) or until the date of end of followup, whichever came first. Follow-up time for all-cause mortality was defined starting from date of blood collection at the two year time point until date of death, or until the last date vital status was updated (COLON: December 2019; EnCoRe: May 2019), or until the date of end of follow-up, whichever came first.

\section{STUDY POPULATION FOR ANALYSES}

In total 1,681 patients were included (COLON: $n=1433$ and EnCoRe: $n=248$ ) between August 2010 and October 2015. Patients with no blood samples available $(n=440)$ and patients with stage IV disease $(n=90)$ or with unknown stage $(n=34)$ were excluded from these analyses. Of all stage I-III CRC patients, 1,117 patients donated a blood sample at diagnosis, whereas 679 (61\%) patients donated blood at diagnosis and at six months as well as two years post-diagnosis (Figure 1). The main reason for not donating blood or filling out questionnaires for follow-up time points was: "too much effort to participate". Another reason for the smaller number of patients for the two year time point is that not all EnCoRe patients had already reached the two year follow-up time point in the study at the moment $25(\mathrm{OH}) \mathrm{D}_{3}$ levels were measured in December 2017. Of all stage I-III patients who donated blood at diagnosis $(n=1117) 68(6 \%)$ died within two years. Of all stage I-III patients who donated blood at diagnosis 95 (9\%) got a recurrence within two years, 34 $(36 \%)$ of them continued in the study and completed the two years measurement. Those 
34 patients were excluded from the analyses, because the outcome would precede the exposure.

We checked whether there were differences in characteristics between patients who donated blood at all three time points $(n=679)$, patients who only donated blood at diagnosis and after six month $(n=270)$ and those who only donated blood at diagnosis $(n=185)$ using descriptive statistics. A few differences were observed between patients with complete data and patients with only blood available at diagnosis. Patients with complete data were slightly younger, were less often smokers and had less often comorbidities. Besides, a lower percentages of deaths, was observed for patients with complete data (10\%) compared to those with incomplete data ( 23 and $36 \%$ ). Patients with complete data also had a lower rate of recurrences within two years (Table S1).

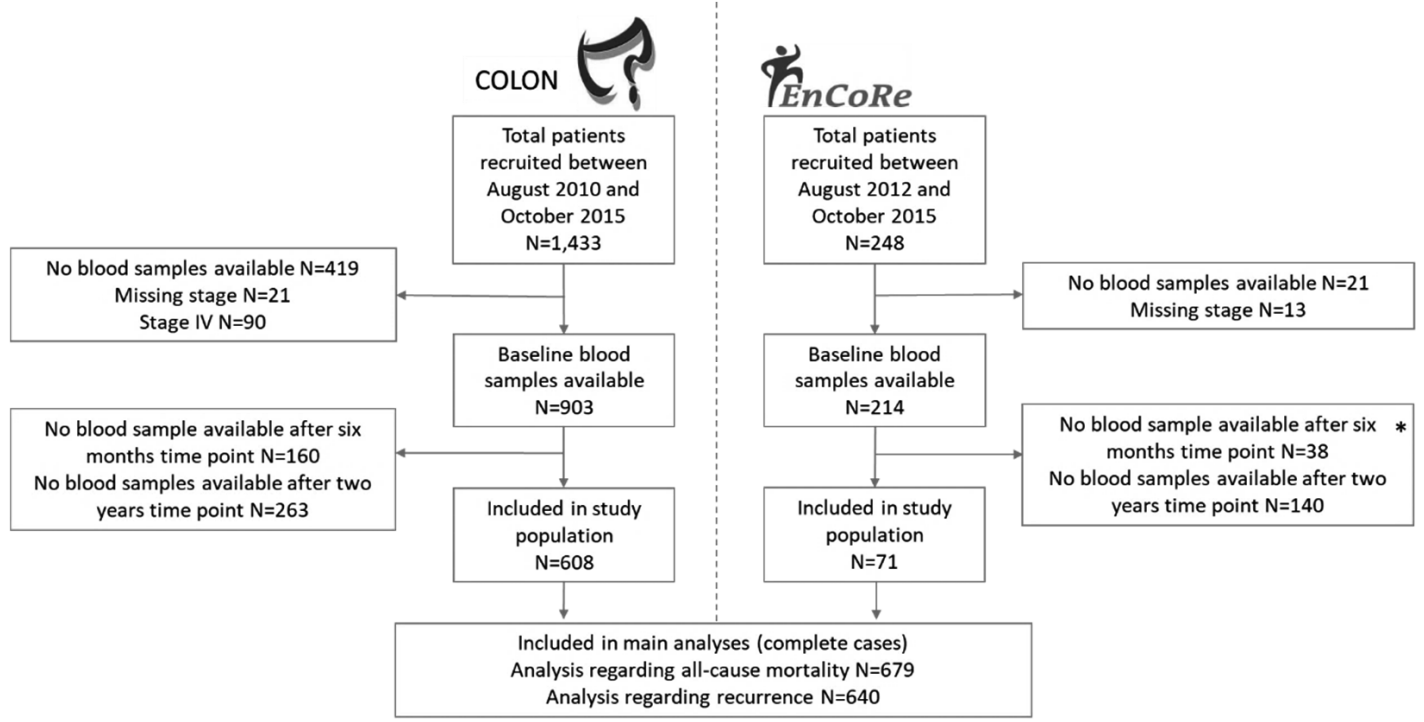

Figure 1: Flowchart of patients included in this study. Patients with complete data (data about serum vitamin D levels available at all time points) were included in the main analyses. *The mean reason for unavailable data of vitamin D levels at the two year time point in the EnCoRe study was that patients had not reached this time point when lab measurements of vitamin $D$ were done.

\section{StATistical ANALYSES}

Patient characteristics at diagnosis were described as medians with interquartile range (IQR) for the total study population and by trajectories of vitamin D levels. To visualize changes in vitamin D levels over time in the different trajectories, generalized linear mixed models (GLMM) were used. Serum $25(\mathrm{OH}) \mathrm{D}_{3}$ as the dependent variable was entered as a continuous variable. Patients, time and time*time were treated as random effect variables. Baseline (diagnosis) was entered as the reference time point. The coefficient for time represents average linear change and the coefficient for time*time captures 
additional quadratic change in $25(\mathrm{OH}) \mathrm{D}_{3}$ levels in $\mathrm{nmol} / \mathrm{L}$. The analysis was adjusted for age, sex, stage, cohort and magnesium intake. Total magnesium intake was entered as time-varying variables. The variables, sex, stage and cohort were entered as fixed factors.

Cox proportional hazard regression analysis was used to assess the association between vitamin D trajectories and recurrence and all-cause mortality in CRC patients. Models were adjusted for age, sex, stage of disease and cohort. Additionally, other potential confounders were tested and included in the model when the HR changed by more than $10 \%$, which only held true for total magnesium intake (cohort-specific quartiles). Smoking (never, former, current), education level (low, medium, high), having comorbidities at diagnosis (y/n), CRC treatment (surgery, chemotherapy, radiotherapy, chemoradiation), mean BMI, mean physical activity level (hours of moderate to vigorous physical activity per week), mean total calcium intake (cohort-specific quartiles), alcohol intake (cohortspecific quartiles), mean fibre intake (cohort-specific quartiles) and energy intake (cohortspecific quartiles) over the two years did not influence the HR by more than $10 \%$ and were thus not included in the models. Log-transformed curves were used for visual inspection of the assumption for the Cox proportional hazard model. No strong evidence of nonparallelism of the log-log curves was observed. Statistical analyses were performed in SAS 9.4 (SAS Institute, Cary NC). P-values $<0.05$ were considered statistically significant.

\section{RESULTS}

The median age of the included patients was 67 (IQR 61-72) years (Table 1). Thirty-five percent of the study population was female. Stage III of disease was most prevalent (41\%). During a median follow-up of 2.2 years for recurrence and 3.5 years for all-cause mortality, 31 and 65 events occurred, respectively. In the total population, a slightly lower $25(\mathrm{OH}) \mathrm{D}_{3}$ level was observed after six months, as compared to diagnosis, and these levels recovered to levels above baseline values after two years (Figure 2; Table 2). When patients were categorized based on their vitamin D trajectories over time, $136(20 \%)$ had consistent deficient 25(OH) $\mathrm{D}_{3}$ levels, 264 (39\%) had consistent sufficient levels, $133(20 \%)$ had increasing levels over time, 90 (13\%) had a temporary drop over time, and $56(8 \%)$ could not be categorized in one of the trajectories, in these patients vitamin D levels were either decreasing over time $(n=34)$ or were higher at six months compared to levels at diagnosis and at two years $(n=22)$. Patients who were consistently deficient had the lowest level of physical activity, the highest BMI, were more often in stage III of disease and consequently received chemotherapy and/or radiotherapy most frequently. The percentages of female patients and supplement users were highest in patients who were consistently sufficient. The percentages of smokers and patients with colon cancer or comorbidities were highest in those patients who followed the trajectory with a temporary drop in levels (Table 1). 

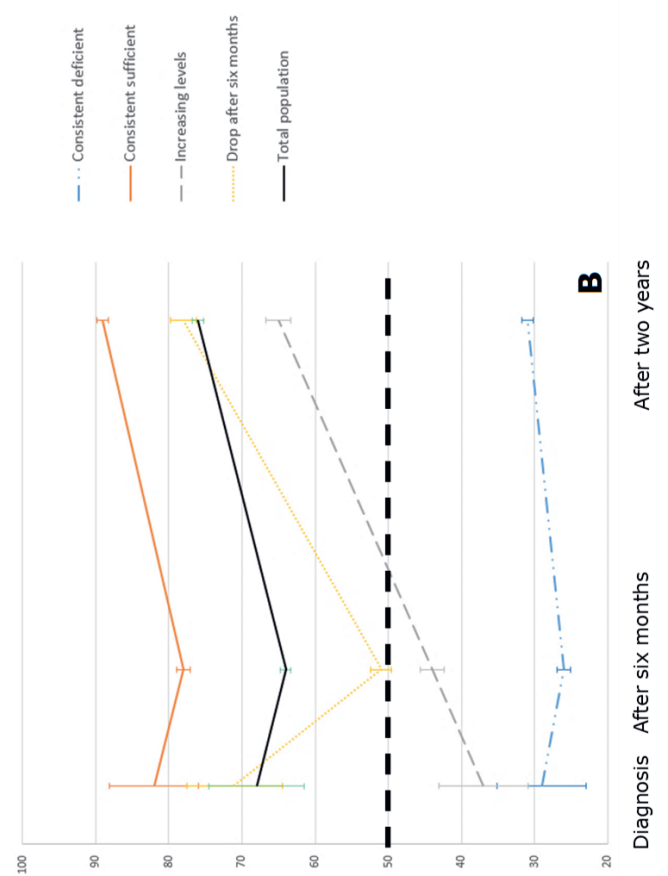

(7//ouu) suo!̣e-ұquәтuо

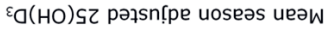

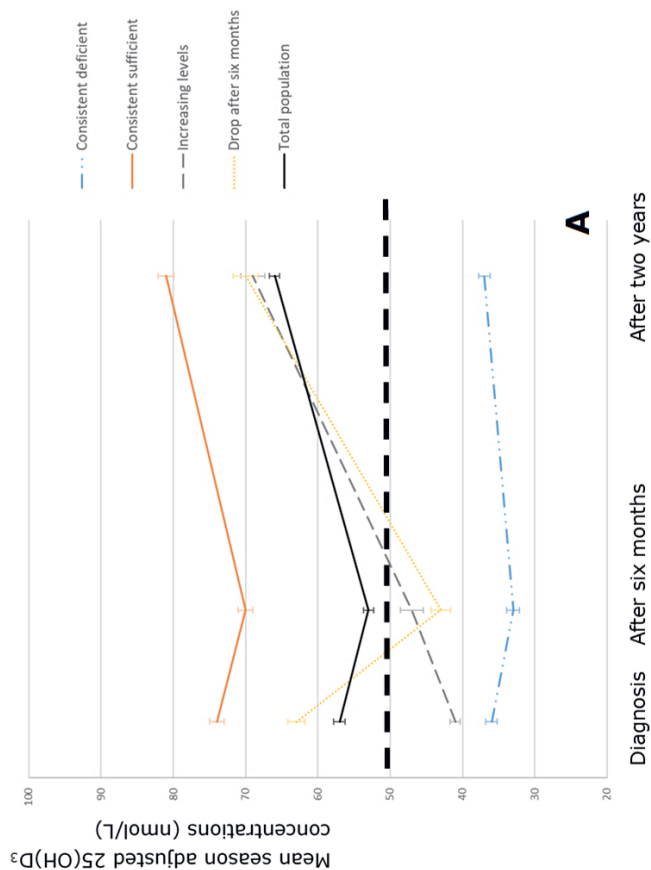

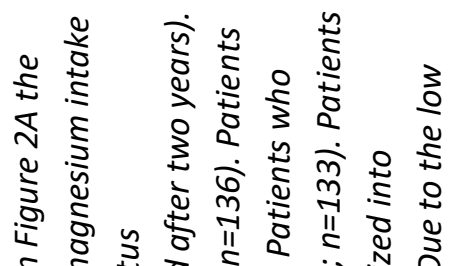

$\leq$ है 300

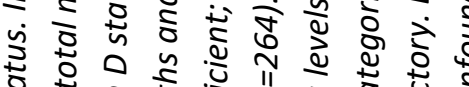

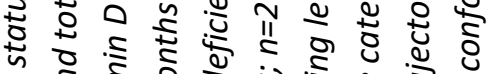

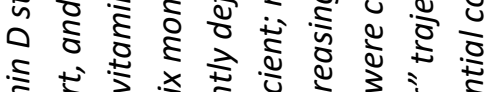

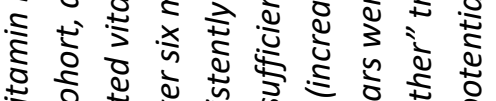

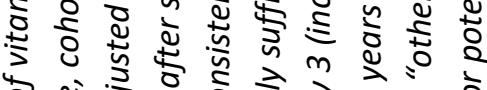

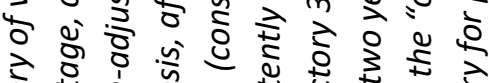

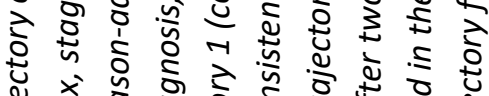

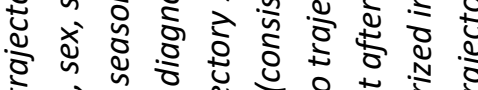
i.

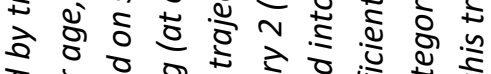

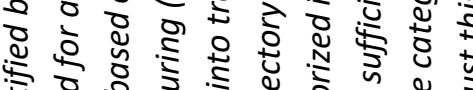
要

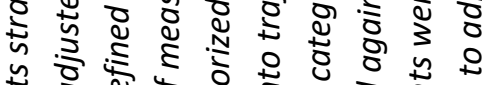

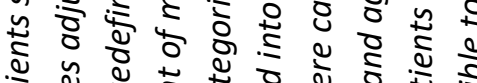

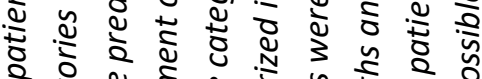
ن

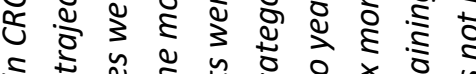

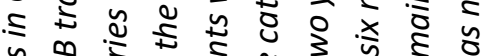

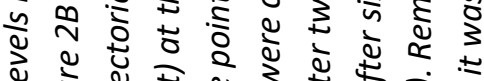

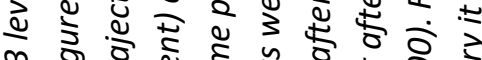
กิ

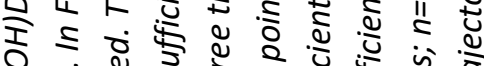

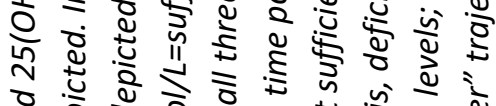

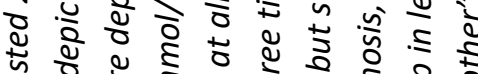
ริ

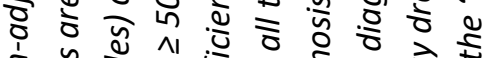
官. ठิ ये पूँ

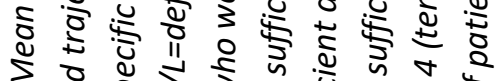

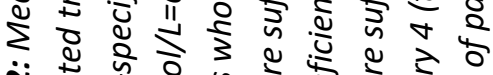

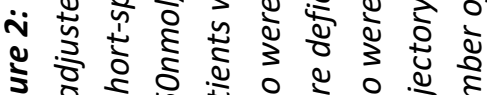

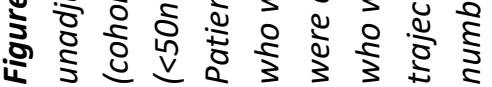




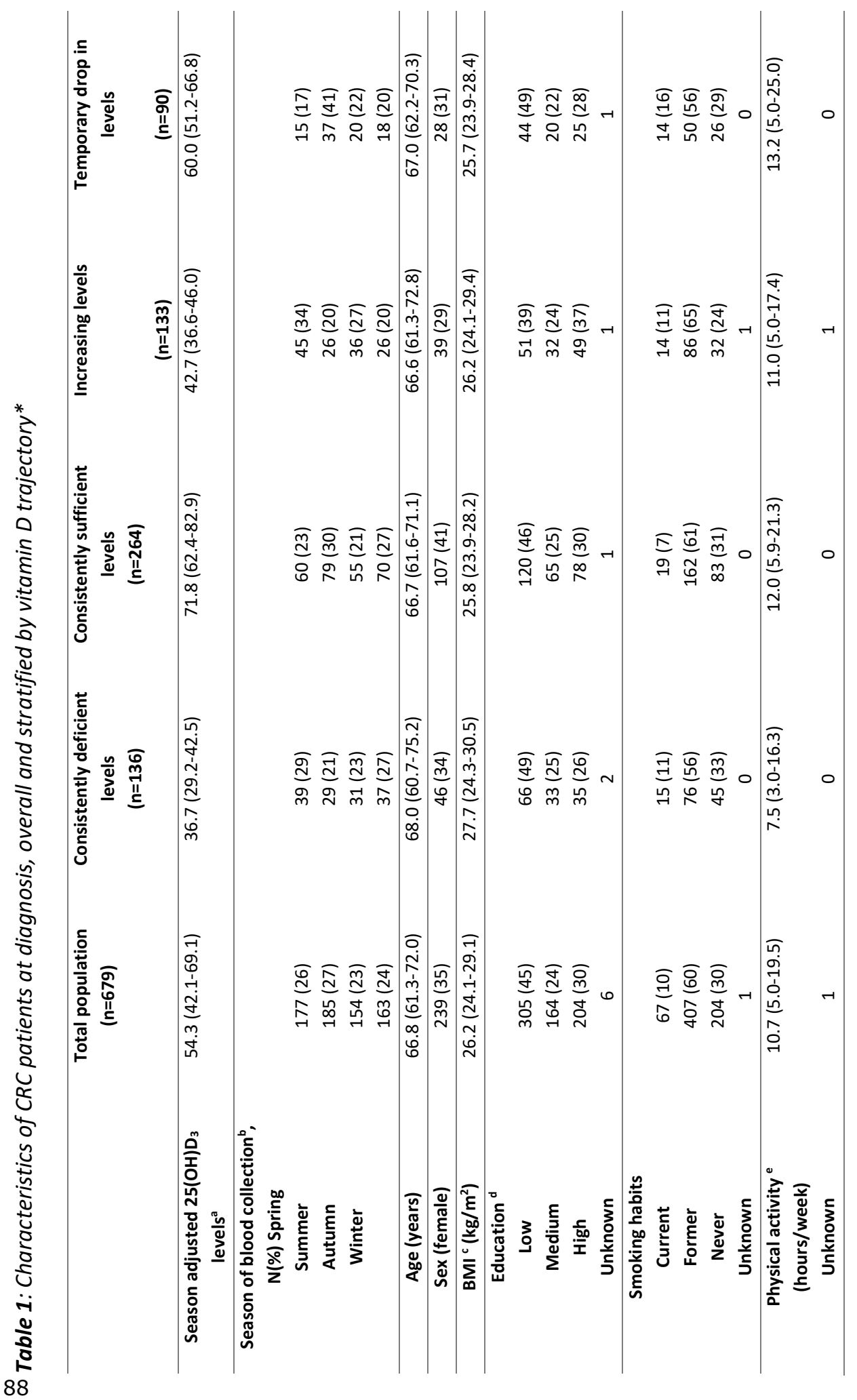




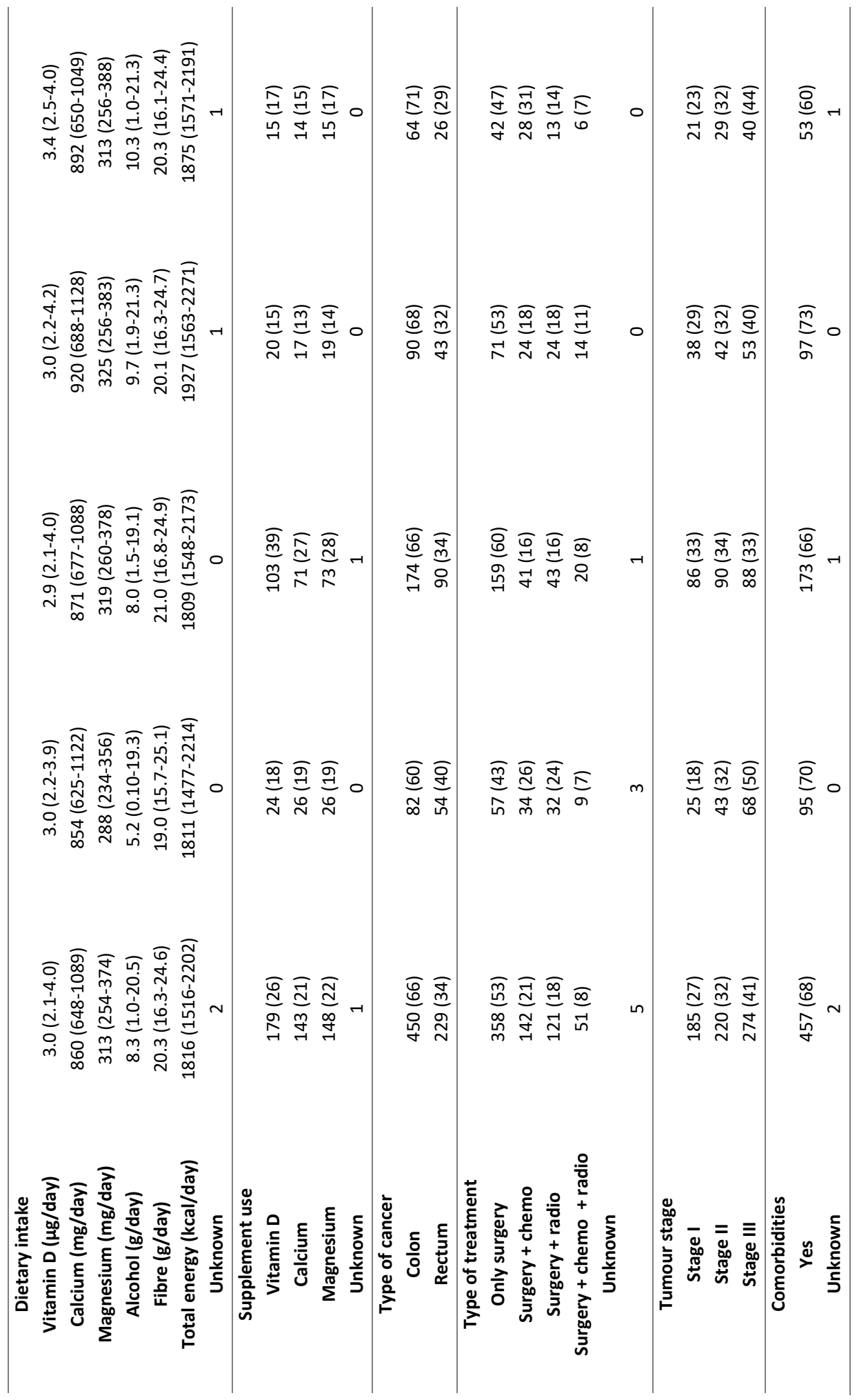




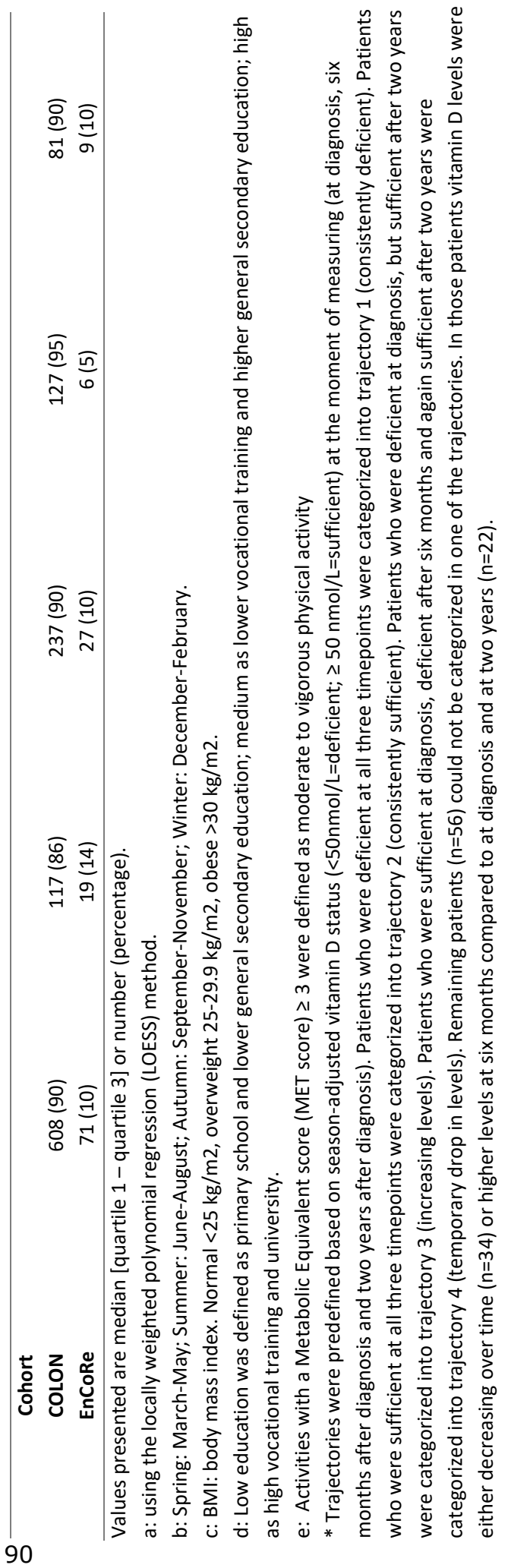




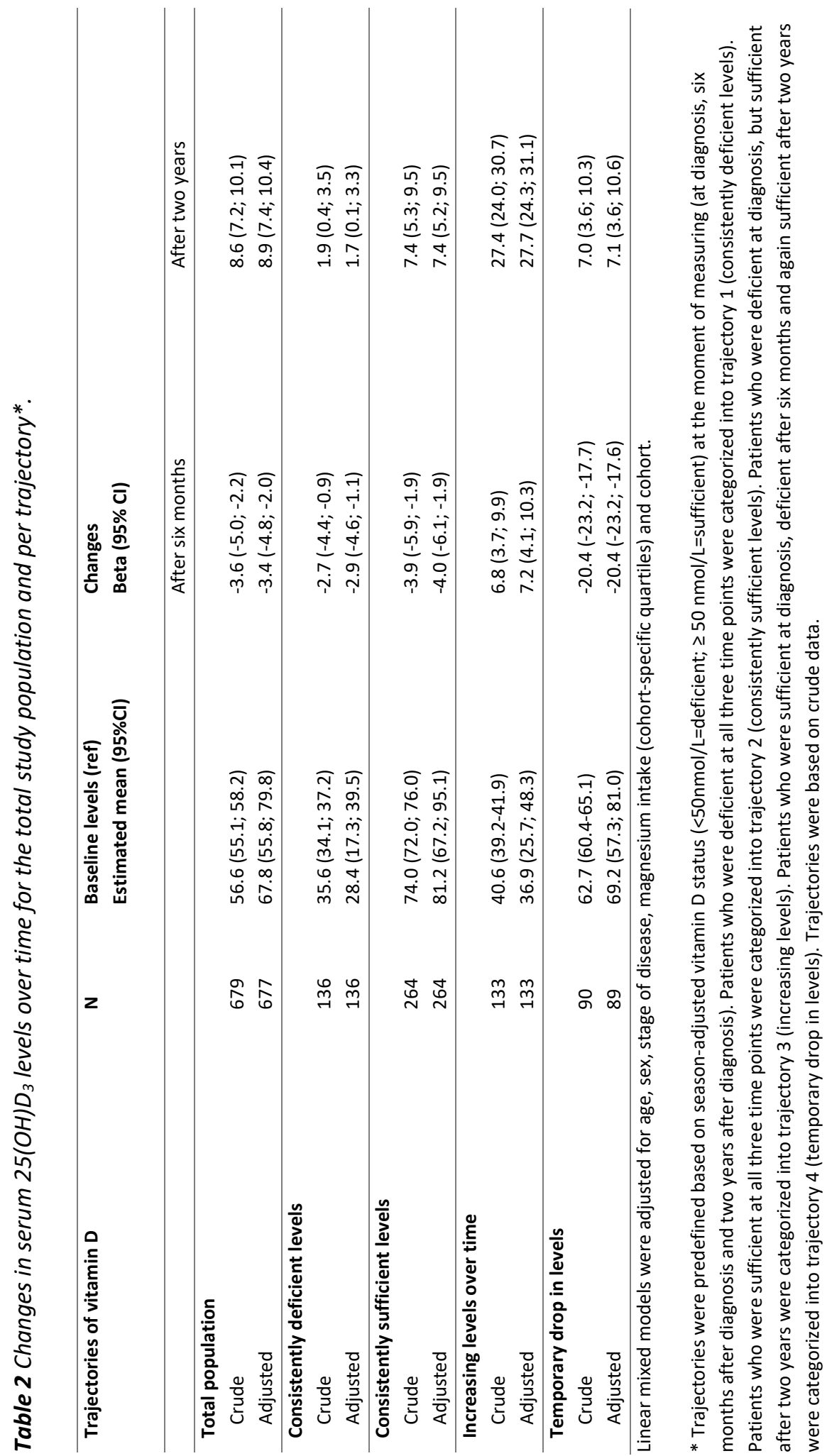




\section{VITAMIN D TRAJECTORIES IN RELATION TO CRC RECURRENCE AND ALL-CAUSE MORTALITY}

Patients who were consistently vitamin D sufficient tended to have a lower risk of recurrence compared to patients who were consistently deficient (HR $0.4095 \% \mathrm{Cl} 0.14$ 1.19) (Table 3). Increasing levels and a temporary drop in levels compared to consistent deficient levels were not associated with the risk of recurrences.

For all-cause mortality, patients who were vitamin D sufficient over time had a $61 \%$ lower risk of all-cause mortality compared to consistently deficient patients (HR $0.3995 \% \mathrm{Cl} 0.21$ 0.73). A lower risk of all-cause mortality was also observed in patients with a temporary drop in levels (HR $0.4195 \% \mathrm{Cl} 0.17-0.94$ ) as compared to patients with consistent deficient levels. Patients with increasing levels also tended to have a lower risk of all-cause mortality compared to consistent deficient levels (HR $0.5595 \% \mathrm{Cl} 0.27-1.10$ ).

Table 3 Association between trajectories of vitamin D over time* and recurrence and allcause mortality

\begin{tabular}{|c|c|c|c|c|}
\hline & $\begin{array}{c}\text { Consistently } \\
\text { deficient } \\
(<50 \mathrm{nmol} / \mathrm{L})\end{array}$ & $\begin{array}{l}\text { Consistently } \\
\text { sufficient } \\
(>50 \mathrm{nmol} / \mathrm{l})\end{array}$ & Increasing levels & $\begin{array}{c}\text { Temporary drop in } \\
\text { levels }\end{array}$ \\
\hline \multicolumn{5}{|c|}{ Risk of CRC recurrence after two years } \\
\hline No./Events & $119 / 8$ & $245 / 6$ & $129 / 9$ & $80 / 6$ \\
\hline \multicolumn{5}{|c|}{ No. of events / 1000} \\
\hline person years & 16 & 6 & 18 & 16 \\
\hline \multicolumn{5}{|l|}{ Crude HR $(95 \% \mathrm{Cl})$} \\
\hline & 1.0 (Ref) & $0.36(0.12-1.03)$ & $0.98(0.37-2.61)$ & $0.82(0.27-2.49)$ \\
\hline \multicolumn{5}{|c|}{ Adjusted $\mathrm{HR}^{\mathrm{a}}(95 \% \mathrm{Cl})$} \\
\hline & 1.0 (Ref) & $0.40(0.14-1.19)$ & $1.02(0.37-2.82)$ & $0.82(0.26-2.56)$ \\
\hline \multicolumn{5}{|c|}{ Risk of all-cause mortality after two years } \\
\hline No./Events & $136 / 26$ & $264 / 17$ & $133 / 12$ & $90 / 7$ \\
\hline \multicolumn{5}{|c|}{ No. of events / 1000} \\
\hline person years & 36 & 12 & 17 & 13 \\
\hline \multicolumn{5}{|l|}{ Crude HR $(95 \% \mathrm{Cl})$} \\
\hline & 1.0 (Ref) & $0.32(0.17-0.58)$ & $0.47(0.24-0.93)$ & $0.36(0.16-0.83)$ \\
\hline \multicolumn{5}{|c|}{ Adjusted $\mathrm{HR}^{\mathrm{a}}(95 \% \mathrm{Cl})$} \\
\hline & 1.0 (Ref) & $0.39(0.21-0.73)$ & $0.54(0.27-1.09)$ & $0.40(0.17-0.93)$ \\
\hline
\end{tabular}

a: Cox proportional hazard model adjusted for age (at diagnosis), sex, stage of disease, cohort, and mean magnesium intake over the two year (cohort-specific quartiles). In the adjusted models 3 patients with missing data for magnesium intake were excluded ( 2 in the increasing levels trajectory and 1 in the temporary drop in levels trajectory). The numbers of events were similar for both the crude and adjusted cox proportional hazard models.

* Trajectories were predefined based on vitamin D status $(<50 \mathrm{nmol} / \mathrm{L}=$ deficient; $\geq 50 \mathrm{nmol} / \mathrm{L}=$ sufficient $)$ at the 
moment of measuring (at diagnosis, six months after diagnosis and two years after diagnosis). Patients who were deficient at all three time points were categorized into trajectory 1 (consistently deficient). Patients who were sufficient at all three time points were categorized into trajectory 2 (consistently sufficient). Patients who were deficient at diagnosis, but sufficient after two years were categorized into trajectory 3 (increasing levels). Patients who were sufficient at diagnosis, deficient after six months and again sufficient after two years were categorized into trajectory 4 (temporary drop in levels).

\section{DISCUSSION}

In the total population, from the moment of CRC diagnosis, a small decrease at six months followed by an increase in $25(\mathrm{OH}) \mathrm{D}_{3}$ levels at two years was observed. Four main trajectories of $25(\mathrm{OH}) \mathrm{D}_{3}$ levels over time were observed 1) consistent deficient levels, 2) consistent sufficient levels, 3) increasing levels and 4) a temporary drop in levels. Patients who had consistent sufficient levels at all time points appeared to have a lower risk of recurrence and had a lower risk of all-cause mortality as compared to those with consistent deficient levels. Also patients with increasing levels or with a temporary drop in $25(\mathrm{OH}) \mathrm{D}_{3}$ levels at six months post-diagnosis appeared to have a lower risk of all-cause mortality compared to patients who had consistent deficient levels.

The small decrease followed by an increase in $25(\mathrm{OH}) \mathrm{D}_{3}$ levels observed in the total population, is in line with the results of a previous study, where also a post-operative fall in $25(\mathrm{OH}) \mathrm{D}_{3}$ levels followed by a recovery was observed in two cohorts combining data of 3,535 CRC patients in total [12].

Consistent sufficient $25(\mathrm{OH}) \mathrm{D}_{3}$ levels compared to consistent deficient $25(\mathrm{OH}) \mathrm{D}_{3}$ levels were associated with a $60 \%$ lower risk of recurrence, although not statistically significantly. It is suggested that higher vitamin D levels decreases the risk of CRC and potentially also CRC recurrence due to its effects on cell differentiation, proliferation and apoptosis [28, 29], as well as due to anti-inflammatory effects [29-31]. In our previous work, in participants of this same pooled COLON and EnCoRe cohort, higher levels of vitamin D were associated with lower levels of interleukin 6 (IL6) [32]. IL6 plays an important role in chronic inflammation [33] and is also suggested to stimulate cancer progression [34-36]. We hypothesized that the risk of recurrences would also be lower in patients with increasing levels of $25(\mathrm{OH}) \mathrm{D}_{3}$. However, no trend was observed for the group of patients with increasing levels nor for those with a temporary drop in levels. This could be due to the low number of events which resulted in a very low power to detect associations (power=70\% with a HR of 0.4). Observational cohort studies with a longer follow-up and/or more events are needed to unravel the association between trajectories of vitamin D levels and recurrence.

All three trajectories characterized by sufficient vitamin D levels at two years after diagnosis seemed to be associated with a lower risk of all-cause mortality compared to the consistent deficient trajectory. A temporary drop in levels was associated with a $61 \%$ 
lower mortality risk compared to consistent deficient levels. In fact, the risk of all-cause mortality was comparable in the group with consistent sufficient levels (60\% lower risk), indicating that a temporary drop, probably due to cancer treatment in particular chemotherapy $[2,15,16]$, may not be associated with a higher risk of all-cause mortality, as long as levels restore afterwards. In addition, increasing levels over time compared to consistent deficient levels appeared to be associated with a lower risk of all-cause mortality. This all together indicates that restoring vitamin D levels may improve all-cause mortality, which stresses the importance of monitoring vitamin D levels over time and optimize levels where needed. On the other hand, it is not clear whether actively improving $25(\mathrm{OH}) \mathrm{D}_{3}$ levels will also improve survival, because low $25(\mathrm{OH}) \mathrm{D}_{3}$ could also be a marker instead of a cause for impaired survival [37]. However, similar trends were observed when patients who died in the first year after the last measurement were excluded (Table S2). This implies that vitamin D is on the causal pathway rather than just a marker of reduced survival.

One of the possible underlying mechanisms for the association between vitamin $D$ and allcause mortality is inflammation [38]. Inflammation plays an important role in cancer progression [39-41] and the active form of vitamin D (1,25-dihydroxycholecalciferol), showed anti-inflammatory properties [29]. Inflammation is also suggested to play a role in the onset of comorbidities such as cardiovascular diseases [42, 43], from which many cancer survivors suffer [44]. We observed no statistical significant associations between trajectories of vitamin D and CRC recurrence. Therefore, it is possible that the observed association of vitamin D with all-cause mortality is not cancer-specific but related to the presence of one or more comorbid conditions. Unfortunately, since we had no data available about the cause of death, due to new strict privacy regulations in the Netherlands, we could not distinguish between cancer-specific mortality and mortality from other causes.

Many studies observed a lower risk of all-cause mortality with higher vitamin D levels at and after diagnosis $[12,13]$. Results of our study also showed beneficial effects of sufficient vitamin D levels over time. Therefore, optimizing vitamin D levels in CRC patients is potentially beneficial. The Health Council of the Netherlands recommends an additional intake of vitamin $D$ by means of supplementation of $10 \mu \mathrm{g} /$ day for women between 50 and 69 years of age and of $20 \mu \mathrm{g} /$ day for all individuals above the age of 70 in the general population $[45,46]$. However, the compliance is low in our study population, $34 \%$ of the female participants and $23 \%$ of the male participants followed the recommendations for vitamin D supplementation. In the Dutch population as a whole, this was $48 \%$ for females and $20 \%$ for males in 2012-2014 [47]. As a first step to improve vitamin D levels on the population level and especially in cancer patients, the already available recommendations should be emphasized by healthcare professionals. 
An important strength of our study relates to the repeated measures of $25(\mathrm{OH}) \mathrm{D}_{3}$ levels. By measuring $25(\mathrm{OH}) \mathrm{D}_{3}$ levels over time in $\mathrm{CRC}$ patients, we were able to assess trajectories over time. This study provides important insight into characteristics of patients with specific trajectories of $25(\mathrm{OH}) \mathrm{D}_{3}$ levels and the associations of those trajectories with cancer outcomes. The main limitation of this study is that only $61 \%$ of all patients included in the COLON and EnCoRe studies completed the two year time point. This limited our power. In addition, we decided to exclude patients who had a recurrence in the first two years after diagnosis, since for those patients the outcome does precede the exposure. This may mean that a population with a better survival prognosis is included in our data analyses. Indeed, patients with complete data were slightly younger, less often smoker and had less often comorbidities. On the other hand, in our previous work, we observed no association between $25(\mathrm{OH}) \mathrm{D}_{3}$ levels at diagnosis and recurrence and a borderline significant association with all-cause mortality $\left(\mathrm{HR}_{\text {per10nmol/L }} 0.9895 \% \mathrm{Cl} 0.90\right.$ $1.07, \mathrm{HR}_{\text {per10nmol/L }} 0.9495 \% \mathrm{Cl} 0.87-1.01$, respectively) [14]. When repeating these analyses within this specific study population, slightly stronger associations were observed $\left(H R_{\text {per10nmol/L }} 0.8695 \% \mathrm{Cl} 0.71-1.05\right.$ for recurrence and $\mathrm{HR}_{\text {per10nmol/L }} 0.8995 \% \mathrm{Cl} 0.78-1.01$ for all-cause mortality). The comparable results indicate that our study population is not a selected population, however our population for analyses seems to have a greater chance of survival compared to CRC patients in general. Therefore, results of this study can only be generalized to stage I-III CRC patients with a relatively good prognosis. Another limitation, is that we did not have enough power to stratify our analyses based on magnesium intake, while we observed an interaction between vitamin D levels and magnesium intake in relation to CRC outcomes in our previous study [14]. Besides, due to the observational nature of our study we cannot prove that high $25(\mathrm{OH}) \mathrm{D}_{3}$ levels over time are the cause of the observed lower risk of all-cause mortality. High vitamin D levels could also be a marker of better health [37] and therefore be associated with a lower risk of all-cause mortality. However, similar trends were observed when patients who died shortly after the end of follow-up were excluded. Furthermore, although corrected for in the analyses, patients who had consistent sufficient $25(\mathrm{OH}) \mathrm{D}_{3}$ levels were more often diagnosed with stage I disease, and had as such a better prognosis. On the other hand, also a lower risk of all-cause mortality was observed in patients in the temporary drop in levels trajectory, while these patients were relatively often diagnosed with stage III disease. Thus the association between $25(\mathrm{OH}) \mathrm{D}_{3}$ levels and all-cause mortality cannot be explained by stage of disease.

In conclusion, all three trajectories with sufficient vitamin D levels after two years appeared to be associated with a lower risk of all-cause mortality compared to the consistent deficient trajectory. Observational studies with a longer follow up and/or events, and consequently more power, are needed to investigate whether trajectories of $25(\mathrm{OH}) \mathrm{D}_{3}$ levels are also associated with a lower risk of long-term cancer recurrence. 


\section{ADDITIONAL INFORMATION}

\section{FUNDING}

The COLON study was financially supported by Wereld Kanker Onderzoek Fonds (WKOF) \& World Cancer Research Fund International (WCRF International) as well as by funds from grant 2014/1179 as part of the World Cancer Research Fund International Regular Grant Programme; Alpe d'Huzes/Dutch Cancer Society (UW 2013-5927, UW 2015-7946); and ERA-NET on Translational Cancer Research (TRANSCAN:Dutch Cancer Society (UW20136397, UW2014-6877) and the Netherlands Organization for Health Research and Development (ZonMw, the Netherlands). The EnCoRe study was supported by grants from the Stichting Alpe d'HuZes within the research program 'Leven met kanker' of the Dutch Cancer Society (Grant No. UM2010-4867 and UM 2012-5653); and ERA-NET on Translational Cancer Research (TRANSCAN:Dutch Cancer Society (UM2014-6877) and by a grant from Kankeronderzoekfonds Limburg as part of Health Foundation Limburg (Grant No. 00005739).

\section{AUTHORS' CONTRIBUTION}

E Wesselink, DE Kok, MJL Bours, HJW de Wilt, MP Weijenberg, E Kampman and FJB van Duijnhoven contributed to the design and the conceptualization of this study. E Wesselink, HJW de Wilt, ETP Keulen, F Kruyt, SO Breukink, EA Kouwenhoven, J van den Ouweland and $M$ van Zutphen contributed to recruitment of participants and the data collection. Statistical data analyses were done by $\mathrm{E}$ Wesselink. The manuscript was drafted by $\mathrm{E}$ Wesselink and FJB van Duijnhoven, and all authors critically read and revised the manuscript. All authors approved the final version of the manuscript.

\section{ACKNOWLEDGEMENT}

The authors would like to thank the participants of the COLON study and the investigators at Wageningen University \& Research and the co-workers from the following hospitals for their involvement in recruitment for the COLON study: Hospital Gelderse Vallei, Ede; Radboudumc, Nijmegen; Slingeland Hospital, Doetinchem; Canisius Wilhelmina Hospital, Nijmegen; Rijnstate Hospital, Arnhem; Gelre Hospitals, Apeldoorn/Zutphen; Hospital Bernhoven, Uden; Isala, Zwolle; ZGT, Almelo; Martini Hospital, Groningen; Admiraal de Ruyter Hospital, Goes/Vlissingen. We would like to thank all participants of the EnCoRe study and the health professionals in the three hospitals involved in the recruitment of participants of the study: Maastricht University Medical Center, VieCuri Medical Center, and Zuyderland Medical Center. We would also like to thank the MEMIC center for data and information management for facilitating the logistic processes and data management of our study. Furthermore, we would like to thank the research dieticians and research 
assistant who are responsible for patient inclusion and follow-up, performing home visits, as well as data collection and processing.

\section{DATA SHARING}

Because the data consist of identifying cohort information, some access restrictions apply, and therefore they cannot be made publicly available. Requests for data of the COLON study can be sent to Dr. Fränzel van Duijnhoven, Division of Human Nutrition and Health, Wageningen University \& Research, the Netherlands (e-mail:

franzel.vanduijnhoven@wur.nl). Requests for data of the EnCoRe study can be sent to Dr. Martijn Bours, Department of Epidemiology, GROW-School for Oncology and Developmental Biology, Maastricht University, the Netherlands (e-mail: m.bours@maastrichtuniversity.nl). 


\section{REFERENCES}

[1] Ng K, Meyerhardt JA, Wu K, Feskanich D, Hollis BW, Giovannucci EL, et al. Circulating 25-hydroxyvitamin d levels and survival in patients with colorectal cancer. Journal of Clinical Oncology. 2008;26:2984-91.

[2] Mezawa H, Sugiura T, Watanabe M, Norizoe C, Takahashi D, Shimojima A, et al. Serum vitamin D levels and survival of patients with colorectal cancer: post-hoc analysis of a prospective cohort study. BMC Cancer. 2010;10:347.

[3] Ng K, Sargent DJ, Goldberg RM, Meyerhardt JA, Green EM, Pitot HC, et al. Vitamin D status in patients with stage IV colorectal cancer: findings from Intergroup trial N9741. Journal of Clinical Oncology. 2011;29:1599-606. [4] Fedirko V, Riboli E, Tjonneland A, Ferrari P, Olsen A, Bueno-de-Mesquita HB, et al. Prediagnostic 25hydroxyvitamin D, VDR and CASR polymorphisms, and survival in patients with colorectal cancer in western European ppulations. Cancer epidemiology, biomarkers \& prevention : a publication of the American Association for Cancer Research, cosponsored by the American Society of Preventive Oncology. 2012;21:582-93.

[5] Tretli S, Schwartz GG, Torjesen PA, Robsahm TE. Serum levels of 25-hydroxyvitamin D and survival in Norwegian patients with cancer of breast, colon, lung, and lymphoma: a population-based study. Cancer Causes Control. 2012;23:363-70.

[6] Zgaga L, Theodoratou E, Farrington SM, Din FV, Ooi LY, Glodzik D, et al. Plasma Vitamin D Concentration Influences Survival Outcome After a Diagnosis of Colorectal Cancer. Journal of Clinical Oncology. 2014;32:2430-9. [7] Wesa KM, Segal NH, Cronin AM, Sjoberg DD, Jacobs GN, Coleton MI, et al. Serum 25-hydroxy vitamin D and survival in advanced colorectal cancer: a retrospective analysis. Nutrition and cancer. 2015;67:424-30.

[8] Facciorusso A, Del Prete V, Muscatiello N, Crucinio N, Barone M. Prognostic role of 25-hydroxyvitamin D in patients with liver metastases from colorectal cancer treated with radiofrequency ablation. Journal of gastroenterology and hepatology. 2016;31:1483-8.

[9] Yang L, Chen H, Zhao M, Peng P. Prognostic value of circulating vitamin D binding protein, total, free and bioavailable 25-hydroxy vitamin D in patients with colorectal cancer. Oncotarget. 2017;8:40214-21.

[10] Maalmi H, Walter V, Jansen L, Chang-Claude J, Owen RW, Ulrich A, et al. Relationship of very low serum 25hydroxyvitamin D3 levels with long-term survival in a large cohort of colorectal cancer patients from Germany. European journal of epidemiology. 2017;32:961-71.

[11] Cooney RV, Chai W, Franke AA, Wilkens LR, Kolonel LN, Le Marchand L. C-reactive protein, lipid-soluble micronutrients, and survival in colorectal cancer patients. Cancer Epidemiology, Biomarkers \& Prevention. 2013;22:1278-88.

[12] Vaughan-Shaw PG, Zgaga L, Ooi LY, Theodoratou E, Timofeeva M, Svinti V, et al. Low plasma vitamin D is associated with adverse colorectal cancer survival after surgical resection, independent of systemic inflammatory response. Gut. 2020;69:103-11.

[13] Maalmi H, Walter V, Jansen L, Boakye D, Schöttker B, Hoffmeister M, et al. Association between Blood 25Hydroxyvitamin D Levels and Survival in Colorectal Cancer Patients: An Updated Systematic Review and MetaAnalysis. Nutrients. 2018;10:896.

[14] Wesselink E, Kok DE, Bours MJL, de Wilt JHW, van Baar H, van Zutphen M, et al. Vitamin D, magnesium, calcium, and their interaction in relation to colorectal cancer recurrence and all-cause mortality. The American journal of clinical nutrition. 2020;111:1007-17.

[15] Wesselink E, Bours MJL, de Wilt JHW, Aquarius M, Breukink SO, Hansson B, et al. Chemotherapy and vitamin D supplement use are determinants of serum 25-hydroxyvitamin D levels during the first six months after colorectal cancer diagnosis. The Journal of steroid biochemistry and molecular biology. 2020;199:105577.

[16] Fakih MG, Trump DL, Johnson CS, Tian L, Muindi J, Sunga AY. Chemotherapy is linked to severe vitamin D deficiency in patients with colorectal cancer. International journal of colorectal disease. 2009;24:219.

[17] Winkels RM, Heine-Broring RC, van Zutphen M, van Harten-Gerritsen S, Kok DE, van Duijnhoven FJ, et al. The COLON study: Colorectal cancer: Longitudinal, Observational study on Nutritional and lifestyle factors that may influence colorectal tumour recurrence, survival and quality of life. BMC Cancer. 2014;14:374.

[18] van Roekel EH, Bours MJ, de Brouwer CP, Ten Napel H, Sanduleanu S, Beets GL, et al. The Applicability of the International Classification of Functioning, Disability, and Health to Study Lifestyle and Quality of Life of 
Colorectal Cancer Survivors. Cancer epidemiology, biomarkers \& prevention : a publication of the American Association for Cancer Research, cosponsored by the American Society of Preventive Oncology. 2014;23:1394405.

[19] Aranow C. Vitamin D and the immune system. Journal of investigative medicine. 2011;59:881-6.

[20] van den Ouweland JM, Beijers AM, van Daal H. Overestimation of 25-hydroxyvitamin D3 by increased ionisation efficiency of 3-epi-25-hydroxyvitamin D3 in LC-MS/MS methods not separating both metabolites as determined by an LC-MS/MS method for separate quantification of 25-hydroxyvitamin D3, 3-epi-25hydroxyvitamin D3 and 25-hydroxyvitamin D2 in human serum. Journal of Chromatography B. 2014;967:195-202. [21] Zhang H, Ahn J, Yu K. Comparing statistical methods for removing seasonal variation from vitamin D measurements in case-control studies. Statistics and its interface. 2011;4:85-93.

[22] Cleveland WS, Devlin SJ. Locally Weighted Regression: An Approach to Regression Analysis by Local Fitting. Journal of the American Statistical Association. 1988;83:596-610.

[23] Holick MF. Vitamin D deficiency. New England Journal of Medicine. 2007;357:266-81.

[24] Koole JL, Bours MJL, Breedveld-Peters JJL, van Roekel EH, van Dongen MCJM, Eussen SJPM, et al. Evaluating the Validity of a Food Frequency Questionnaire in Comparison with a 7-Day Dietary Record for Measuring Dietary Intake in a Population of Survivors of Colorectal Cancer. Journal of the Academy of Nutrition and Dietetics. 2020;120:245-57.

[25] Dutch Nutrition Center. NEVO Nederlandse Voedingsmiddelen tabel 2011 (In Englisch: Dutch Food Composition Table).

[26] Wendel-Vos GC, Schuit AJ, Saris WH, Kromhout D. Reproducibility and relative validity of the short questionnaire to assess health-enhancing physical activity. Journal of clinical epidemiology. 2003;56:1163-9. [27] Van Leersum N, Snijders H, Henneman D, Kolfschoten N, Gooiker G, Ten Berge M, et al. The Dutch surgical colorectal audit. European Journal of Surgical Oncology (EJSO). 2013;39:1063-70.

[28] Feldman D, Krishnan AV, Swami S, Giovannucci E, Feldman BJ. The role of vitamin D in reducing cancer risk and progression. Nature reviews Cancer. 2014;14:342-57.

[29] Krishnan AV, Feldman D. Mechanisms of the anti-cancer and anti-inflammatory actions of vitamin D. Annual review of pharmacology and toxicology. 2011;51:311-36.

[30] Ferrer-Mayorga G, Larriba MJ, Crespo P, Munoz A. Mechanisms of action of vitamin D in colon cancer. The Journal of steroid biochemistry and molecular biology. 2018;185:1-6.

[31] Liu W, Zhang L, Xu H-J, Li Y, Hu C-M, Yang J-Y, et al. The anti-inflammatory effects of vitamin D in tumorigenesis. International journal of molecular sciences. 2018;19:2736.

[32] Wesselink E, Balvers M, Bours MJL, de Wilt JHW, Witkamp RF, van Baar H, et al. The association between circulating levels of vitamin $\mathrm{D}$ and inflammatory markers in the first 2 years after colorectal cancer diagnosis. Therapeutic Advances in Gastroenterology. 2020;13:1756284820923922.

[33] Gabay C. Interleukin-6 and chronic inflammation. Arthritis research \& therapy. 2006;8:S3.

[34] Klampfer L. Cytokines, inflammation and colon cancer. Current cancer drug targets. 2011;11:451-64.

[35] Knupfer H, Preiss R. Serum interleukin-6 levels in colorectal cancer patients--a summary of published results. International journal of colorectal disease. 2010;25:135-40.

[36] Brozek W, Bises G, Girsch T, Cross HS, Kaiser HE, Peterlik M. Differentiation-dependent expression and mitogenic action of interleukin- 6 in human colon carcinoma cells: relevance for tumour progression. European journal of cancer. 2005;41:2347-54.

[37] Autier P, Boniol M, Pizot C, Mullie P. Vitamin D status and ill health: a systematic review. The lancet Diabetes \& endocrinology. 2014;2:76-89.

[38] van Harten-Gerritsen AS, Balvers MG, Witkamp RF, Kampman E, van Duijnhoven FJ. Vitamin D, inflammation and colorectal cancer progression: A review of mechanistic studies and future directions for epidemiological studies. Cancer Epidemiology and Prevention Biomarkers. 2015:cebp. 0601.2015.

[39] Hanahan D, Weinberg RA. Hallmarks of cancer: the next generation. Cell. 2011;144:646-74.

[40] Lasry A, Zinger A, Ben-Neriah Y. Inflammatory networks underlying colorectal cancer. Nature immunology. $2016 ; 17: 230$. 
[41] Seruga B, Zhang H, Bernstein L, Tannock IF. Cytokines and their relationship to the symptoms and outcome of cancer. Nature Reviews Cancer. 2008;8:887.

[42] Alfano CM, Peng J, Andridge RR, Lindgren ME, Povoski SP, Lipari AM, et al. Inflammatory cytokines and comorbidity development in breast cancer survivors versus noncancer controls: evidence for accelerated aging? Journal of Clinical Oncology. 2017;35:149.

[43] Lopez-Candales A, Burgos PMH, Hernandez-Suarez DF, Harris D. Linking chronic inflammation with cardiovascular disease: from normal aging to the metabolic syndrome. Journal of nature and science. 2017;3. [44] Bluethmann SM, Mariotto AB, Rowland JH. Anticipating the "silver tsunami": prevalence trajectories and comorbidity burden among older cancer survivors in the United States. AACR; 2016. p. 1029-36.

[45] Counsel DH. Dossier Evaluatie van de voedingsnormen voor vitamine D. 2012.

[46] EFSA Panel on Dietetic Products N, Allergies. Dietary reference values for vitamin D. EFSA Journal. 2016;14:e04547.

[47] Rossum Cv, Buurma E, Vennemann F, Beukers M, Drijvers J, Ocke M. Food consumption in 2012-2014 compared to Guidelines for healthy food 2015. Rijksinstituut voor Volksgezondheid en Milieu RIVM; 2017. 


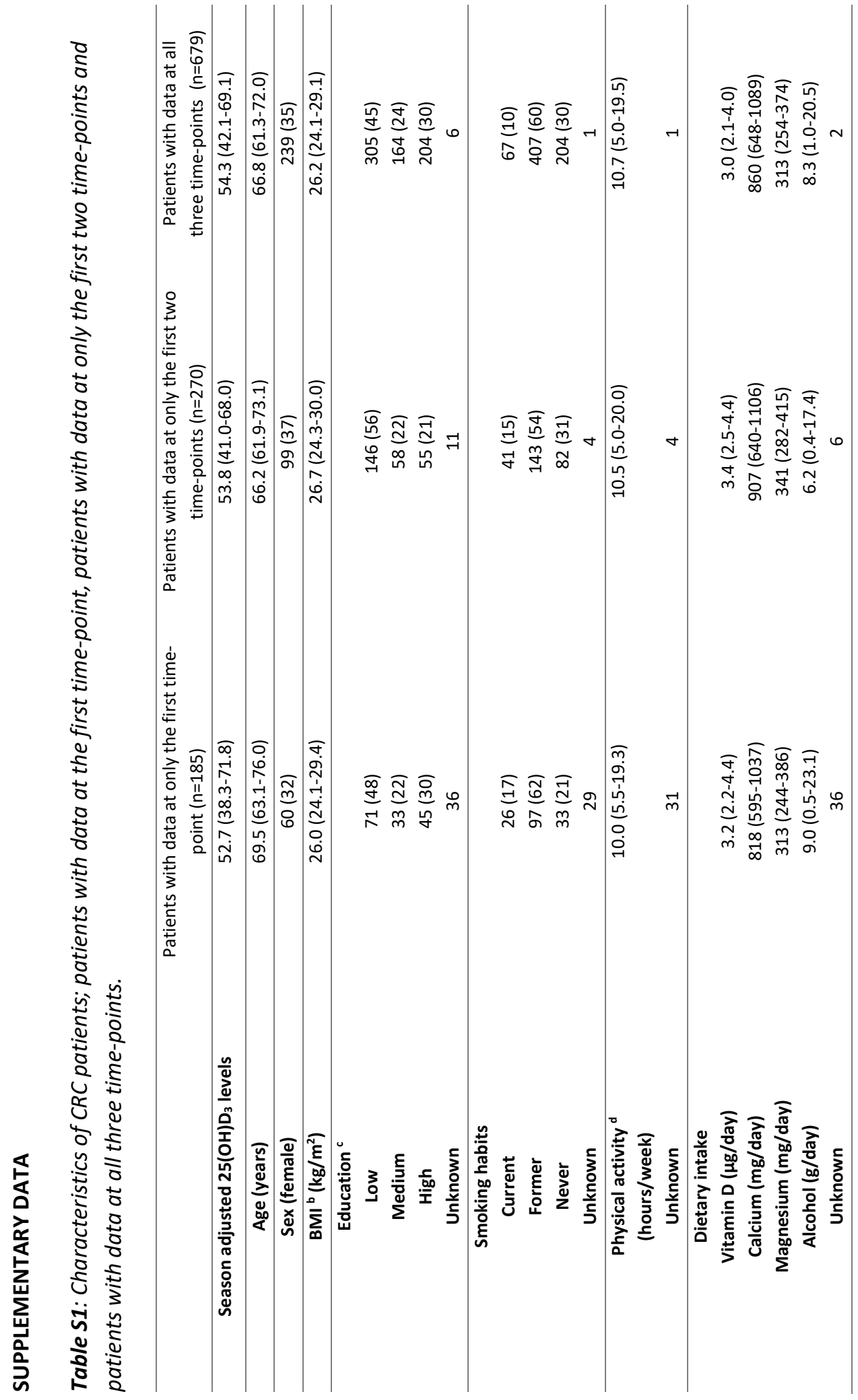


CHAPTER 4

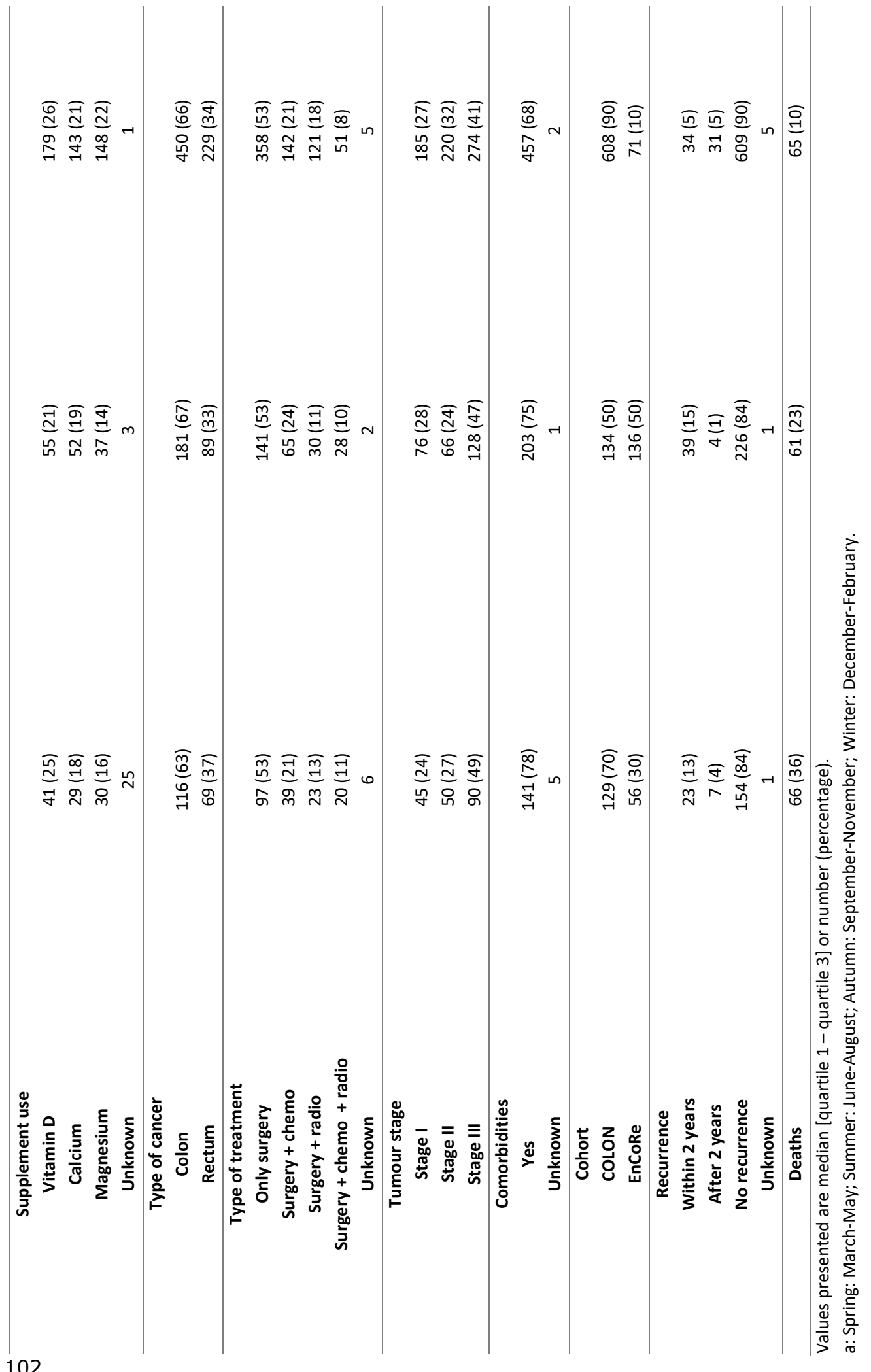




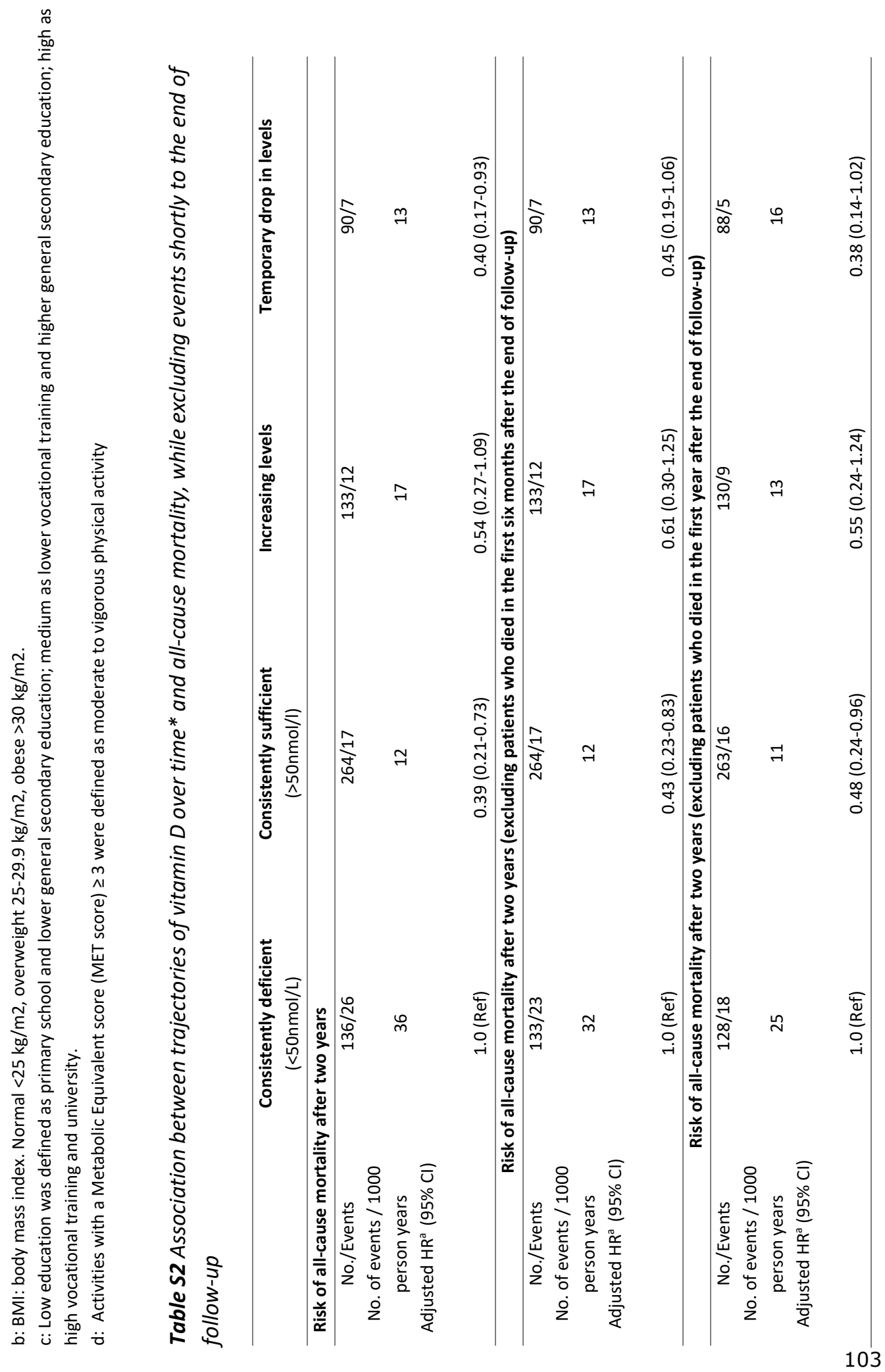


CHAPTER 4

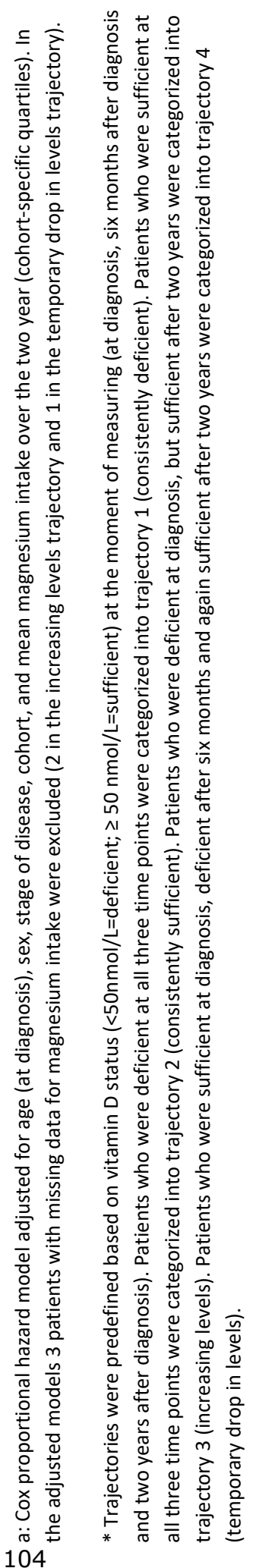





\title{
CHAPTER 5
}

\section{THE ASSOCIATION BETWEEN CIRCULATING LEVELS OF VITAMIN D AND INFLAMMATORY MARKERS IN THE FIRST TWO YEARS AFTER COLORECTAL CANCER DIAGNOSIS}

\author{
Evertine Wesselink \\ Michiel G.J. Balvers \\ Martijn J.L. Bours \\ Johannes H.W. de Wilt \\ Renger F. Witkamp \\ Harm van Baar \\ Anne J.M.R. Geijsen \\ Henk van Halteren \\ Eric T.P. Keulen \\ Dieuwertje E. Kok \\ Ewout A. Kouwenhoven \\ Jody van den Ouweland \\ Moniek van Zutphen \\ Matty P. Weijenberg \\ Ellen Kampman
}

Franzel J.B. van Duijnhoven

Therapeutic Advances in Gastroenterology. 2020 May 27;13:1756284820923922. 


\section{ABSTRACT}

Background: Calcitriol, the active form of vitamin D, may inhibit colorectal cancer (CRC) progression, which has been mechanistically linked to an attenuation of a proinflammatory state. The present study investigated the associations between circulating twenty-five hydroxy vitamin $\mathrm{D}_{3}\left(25(\mathrm{OH}) \mathrm{D}_{3}\right)$ levels and inflammatory markers (IL10, IL8, IL6, TNF $\alpha$ and hsCRP) in the two years following CRC diagnosis.

Methods: Circulating $25(\mathrm{OH}) \mathrm{D}_{3}$ levels and inflammatory markers were assessed at diagnosis, after six, 12 and 24 months from 798 patients with sporadic CRC participating in two prospective cohort studies. Associations between $25(\mathrm{OH}) \mathrm{D}_{3}$ levels and individual inflammatory markers as well as a summary inflammatory z-score were assessed at each time point by multiple linear regression analyses. To assess the association between $25(\mathrm{OH}) \mathrm{D}_{3}$ and inflammatory markers over the course of two years, linear mixed model regression analyses were conducted.

Results: Higher $25(\mathrm{OH}) \mathrm{D}_{3}$ levels were associated with lower IL6 levels at diagnosis, at six months after diagnosis and over the course of two years $(\beta-0.0695 \% \mathrm{Cl}-0.08 ;-0.04)$. In addition, $25(\mathrm{OH}) \mathrm{D}_{3}$ levels were inversely associated with the summary inflammatory zscore at diagnosis and over the course of two years $(\beta-0.1795 \% \mathrm{Cl}-0.25 ;-0.08)$. Also a significant inverse association between $25(\mathrm{OH}) \mathrm{D}_{3}$ levels and IL10 was found over the course of two years. Intra-individual analyses showed an inverse association between $25(\mathrm{OH}) \mathrm{D}_{3}$ and IL10, IL6 and TNF $\alpha$. No statistically significant associations between $25(\mathrm{OH}) \mathrm{D}_{3}$ and IL8 and hsCRP levels were observed.

Conclusions: Serum $25(\mathrm{OH}) \mathrm{D}_{3}$ levels were inversely associated with the inflammatory zscore and in particular with IL6 in the years following CRC diagnosis. This is of potential clinical relevance as IL6 has an important role in chronic inflammation and is also suggested to stimulate cancer progression. Further observational studies should investigate whether a possible $25(\mathrm{OH}) \mathrm{D}_{3}$-associated reduction of inflammatory mediators influences treatment efficacy and CRC recurrence.

Keywords: $25(\mathrm{OH}) \mathrm{D}_{3}$, inflammatory markers, colorectal cancer, interleukin 6, cytokines 


\section{INTRODUCTION}

Inflammation is defined as one of the enabling hallmarks of cancer [1]. It is estimated that over $20 \%$ of all cancers develop as a direct consequence of systemic low grade inflammation [2,3]. Different inflammatory markers, among which cytokines, can stimulate cancer progression and enhance tumour invasion and metastasis in many cancers including colorectal cancer (CRC) [4, 5]. Also, intrinsic inflammatory processes by the tumour itself are involved in the majority of colorectal tumours [5]. In addition, higher levels of inflammatory markers are associated with advanced disease $[6,7]$ and worse CRC outcomes $[8,9]$. The use of non-steroidal anti-inflammatory drugs, in particular aspirin, has been shown to reduce CRC risk and potentially CRC recurrence [10]. Given the important role of inflammation in the development and progression of $\mathrm{CRC}$, preventing or reversing systemic low grade inflammation is considered a relevant and promising approach to improve CRC prognosis.

The active form of vitamin $\mathrm{D}, 1,25$-dihydroxycholecalciferol $\left(1,25(\mathrm{OH})_{2} \mathrm{D}_{3}\right)$, showed antiinflammatory actions in several cancer models, including CRC models [11, 12]. As a consequence, modulation of inflammatory responses by vitamin $\mathrm{D}$ could probably result in improved cancer prognosis. Vitamin $D$ is derived from the production of vitamin $D_{3}$ in the skin, via a non-enzymatic two-step process induced by UVB radiation and heat, or obtained from the diet [13]. In the liver, vitamin D is converted by 25 -hydroxylase to twenty-five hydroxy vitamin $\mathrm{D}_{3}\left(25(\mathrm{OH}) \mathrm{D}_{3}\right)$, the main circulating form of vitamin $\mathrm{D}$ and the most reliable measurement of an individual's vitamin D status [14]. Finally, $25(\mathrm{OH}) \mathrm{D}_{3}$ is converted to its active form $1,25(\mathrm{OH})_{2} \mathrm{D}_{3}$ by 1- $\alpha$ hydroxylase mainly in the kidney [13]. Two main mechanisms by which $1,25(\mathrm{OH}) \mathrm{D}_{3}$ exerts anti-inflammatory responses are suggested. Firstly, $1,25(\mathrm{OH})_{2} \mathrm{D}_{3}$ may inhibit Nuclear Factor kappa B (NFkB) signalling [11]. NFkB is an important transcription factor involved in the regulation of innate immune responses and inflammation, activation results in the production of pro-inflammatory cytokines [15]. $N F K B$ is suggested to be involved in CRC progression [16]. Secondly, $1,25(\mathrm{OH})_{2} \mathrm{D}_{3}$ may suppress p38 stress kinase signalling through the upregulation of mitogen-activated protein kinase phosphate 5 , resulting in an inhibition of pro-inflammatory cytokine production, such as interleukin (IL)-6, IL8 and tumour necrosis factor (TNF)- $\alpha$ [11].

Despite the suggested mechanistic basis described above, only a few studies directly examined the associations between circulating vitamin D levels and levels of inflammatory markers [17-20]. Results of a recent study in CRC patients showed a weak correlation between $25(\mathrm{OH}) \mathrm{D}_{3}$ and C-reactive protein (CRP) levels [20]. In addition, results of a study in severely obese individuals showed an inverse association between $25(\mathrm{OH}) \mathrm{D}_{3}$ levels and high sensitivity C-reactive protein (hSCRP), IL6 and TNF $\alpha$ levels. In addition, a study in colorectal adenoma patients found a non-statistically significant decline in hsCRP, IL6, IL1 $\beta$ and TNF $\alpha$ levels and a significantly lower summary inflammatory z-score after vitamin D 
supplementation [17]. To our knowledge, only one study investigated the association between $25(\mathrm{OH}) \mathrm{D}_{3}$ levels and inflammatory markers in CRC patients before and after cancer treatment [20]. However, in this study only CRP was examined. The increasing insight that specific inflammatory pathways are involved in cancer progression $[5,21]$ merits more detailed assessment of individual cytokines. It is important to obtain more insight into the systemic inflammatory status before and after cancer treatment and the question whether circulating vitamin D levels are associated with the systemic inflammatory status in CRC patients. The aim of this study was to investigate the association between serum $25(\mathrm{OH}) \mathrm{D}_{3}$ levels and plasma inflammatory markers involved in CRC progression $[21,22]$ before and after treatment for CRC.

\section{METHODS}

\section{STUDY POPULATION}

In total, 798 CRC patients were included, recruited between October 2013 and November 2016, from two prospective cohort studies in the Netherlands; the COLON study $(n=564)$ and the EnCoRe study $(n=234)$. These patients donated blood samples at diagnosis and several time points before and after treatment.

The design of the COLON study [23] (NCT03191110) as well as that of the EnCoRe study [24] (NTR7099) has been described earlier. In short, newly diagnosed CRC patients were recruited directly after diagnosis in 14 hospitals and were followed during and after treatment. Men and women above the age of 18 were eligible. In the COLON study patients with stage I-IV CRC were eligible. In the EnCoRe study patients with stage IV of disease were not recruited. Non-Dutch speaking patients, or patients with a history of CRC or (partial) bowel resection, chronic inflammatory bowel disease, hereditary CRC syndromes (e.g. Lynch syndrome, Familial Adenomatous Polyposis, Peutz-Jegher), dementia or another mental condition obstructing participation were excluded from the study. All patients signed informed consent and the COLON study was approved by the Committee on Research involving Human Subjects, region Arnhem-Nijmegen, the Netherlands (2009-349). The EnCoRe study was approved by the Medical Ethics Committee of the University Hospital Maastricht and Maastricht University, the Netherlands (METC 11-3-075).

\section{BLOOD COLLECTION}

For the COLON study, blood samples were obtained during a regular clinical visit in the hospital at diagnosis, and at six months and two years after diagnosis. For patients receiving chemotherapy blood samples were also drawn in the hospital one year after diagnosis. For the EnCoRe study, blood samples were obtained during a home visit before the start of treatment and at six weeks, six months, one year and two years after the end 
of treatment. All blood samples were centrifuged and aliquoted into serum and plasma and immediately stored in a freezer at $-80^{\circ} \mathrm{C}$ until analysis.

To be able to harmonize the data of both cohorts for analyses at several time points after CRC diagnosis, we selected the time point in the EnCoRe cohort closest to either six months (mean 7.6 months), one year (mean 12.6 months) and two years (mean 23.8 months) after diagnosis as the second, third and fourth time points for these analyses (Supplementary data Figure S1).

\section{SERUM VITAMIN D LEVELS}

Serum $25(\mathrm{OH}) \mathrm{D}_{3}$ levels were measured for all participants by liquid chromatography tandem mass spectrometry (LC-MS/MS) in the Canisius Wilhelmina Hospital, Nijmegen, the Netherlands [25]. The inter-assay coefficients of variation were $5.3 \%, 3.1 \%$ and $2.9 \%$ at $25(\mathrm{OH}) \mathrm{D}_{3}$ concentrations of $39.0,92.5$ and $127.0 \mathrm{nmol} / \mathrm{L}$, respectively and were calculated from QC data over at least 30 days from the same lot that was used for measurement of the study samples. Serum $25(\mathrm{OH}) \mathrm{D}_{3}$ is the main circulating form of vitamin $D$ and the most reliable measurement of an individual's vitamin D status [14].

\section{PLASMA INFLAMMATORY CYTOKINES}

Plasma levels of IL-1 $\beta$, IL6, IL8, IL10, IL-12p70 and TNF $\alpha$ were determined using a custommade multiplex assay using electrochemiluminiscence detection (Meso Scale Diagnostics, Rockville, Maryland, USA). The analyses were performed following the manufacturers' instructions, and assay plates were analysed on a QuickPlex SQ 120 plate reader (Meso Scale Diagnostics). Each sample plate contained a calibration curve and three manufacturer's quality control (QC) samples with different levels of cytokines. Calibrators, QCs and study samples were analysed in duplicate. Control samples were not masked because the laboratory technician performs the initial quality assessment, and therefore needs to identify the QCs. However, the study samples were blinded.

Cytokines were previously shown to remain stable in plasma for a period up to 2 years of storage at $-80^{\circ} \mathrm{C}[26]$. Therefore, only samples stored $<2$ years were analysed. In addition, to exclude any residual influence of storage time on cytokine levels, levels for each individual were measured after storage for a fixed time period; in this way storage time did not influence relative levels of cytokines in each individual over time. For example, all samples of patient $X$ were analysed after approximately 3 months of storage and all samples of patient $Y$ after approximately 4 months of storage. In total, we had four analysis rounds i.e. February 2016, May 2016, January 2017 and December 2017, consisting of in total 70 assay plates. 
The quality of the multiplex cytokine data was monitored by evaluating the inter-batch reproducibility of the manufacturers' QC samples for which target values were provided. IL12p70 and IL1 $\beta$ were excluded for further analyses because the plasma levels were undetectable in most of the samples. Inter and intra-batch coefficients of variation for IL10, IL8, IL6 and TNF $\alpha$ were $<8 \%$, and reported values deviated no more than $15 \%$ from the assigned target values.

The results for individual samples within the calibration range with a coefficient of variation above $40 \%$ were considered too imprecise to be further processed. Levels measured between the lower detection limit of a specific plate and the lowest point of the calibration line $(0.4 \%)$ were imputed as the lowest detection limit of all plates. Nondetectable levels $(0.1 \%)$ were imputed as the lowest detection limit of all plates divided by 2.

High sensitive C-reactive protein (hsCRP) was measured at diagnosis, six months after diagnosis and one year after diagnosis, using an immuno-MALDI mass spectrometry method [27] (BEVITAL, Bergen, Norway). The inter-assay coefficient ranged between 3-6\%.

\section{Summary inflammatory z-score}

The summary inflammatory z-score (including IL10, IL8, IL6, TNF $\alpha$ and hSCRP) was calculated as follows [17]. First, a normalized $z$-score for each individual biomarker value, with a mean of zero and standard deviation of 1.0 was calculated as $\mathrm{z}_{\mathrm{j}}=\left(\mathrm{x}_{\mathrm{ij}}-\mu_{\mathrm{j}}\right) / \sigma_{\mathrm{j}}$, where $\mathrm{x}_{\mathrm{ij}}$ is a participant's (i) inflammation marker value at a given visit (j), and $\mu_{\mathrm{j}}$ and $\alpha_{\mathrm{j}}$ are the study population mean and standard deviation at given visits, respectively. The combined score was calculated by summing the z-scores of each inflammatory marker (inflammatory $z$-score $=z_{\text {score }}\left(\right.$ LnIL10) $+z_{\text {score }}\left(\right.$ LnIL8) $+z_{\text {score }}($ LnIL6) $)+z_{\text {score }}($ LnTNF $\alpha)+$ $\left.z_{\text {score }}(\operatorname{LnCRP})\right)$. This summary inflammatory z-score was calculated to cluster conceptually related markers of low-grade inflammation and improve statistical efficiency.

\section{DATA COLLECTION}

Information on demographics (age, gender, education), menopausal status and lifestyle (smoking, use of non-steroidal anti-inflammatory drugs (NSAIDs) was obtained using selfadministered questionnaires in both cohorts at the same time as the blood samples were collected. Information on height, weight, and waist and hip circumference was collected using self-administered questionnaires in the COLON study. In the EnCoRe study, these measurements were performed during home visits. Physical activity was assessed using the Short QUestionnaire to ASsess Health-enhancing physical activity (SQUASH) in both cohorts [28]. 
Habitual dietary intake in the month (COLON study) or year (EnCoRe study) preceding diagnosis was assessed using a semi-quantitative food frequency questionnaire. During follow-up habitual dietary intake was assessed with the same semi-quantitative food frequency questionnaire in the COLON study and 7 day food diaries in the EnCoRe study. Average daily intake of macro and micro-nutrients was calculated using the 2011 Dutch food composition table (NEVO-table, 2011).

Clinical data, such as stage of disease, tumour location (colon/rectum), tumour differentiation, histological type, date of start treatment, type of treatment (surgery, neoadjuvant/adjuvant chemotherapy, radiation therapy) and presence of comorbidities (diabetes, endocrine disorders, cardiovascular, infectious, gastro-intestinal, muscular and joint, neurologic, pulmonary and urogenital diseases) for both cohorts were derived from the Dutch ColoRectal Audit (DCRA). This nationwide audit was initiated by the association of surgeons of the Netherlands to monitor, evaluate and improve CRC care [29].

\section{DATA ANALYSES}

Levels on inflammatory markers (IL10, IL8, IL6, TNF $\alpha$ and hsCRP) were natural logtransformed to obtain normally distributed data.

Patient characteristics at diagnosis were described as numbers with percentages or medians with interquartile range (IQR) for the total study population and stratified by vitamin D status (insufficiency serum $25(\mathrm{OH}) \mathrm{D}_{3}<50 \mathrm{nmol} / \mathrm{L}$ and sufficiency serum $\left.25(\mathrm{OH}) \mathrm{D}_{3} \geq 50 \mathrm{nmol} / \mathrm{L}\right)[30]$. In addition, levels of serum $25(\mathrm{OH}) \mathrm{D}_{3}$ and plasma inflammatory markers at diagnosis and at the follow up time points were described as medians with IQR. Sensitivity analyses were done for patients who donated blood samples at all measurement points during the study period.

The association between serum $25(\mathrm{OH}) \mathrm{D}_{3}$, continuous per $10 \mathrm{nmol} / \mathrm{L}$, and inflammatory markers was assessed using multivariable linear regression analyses. This was done for each inflammatory marker separately as well as for the summary inflammatory z-score.

Based on literature the following covariates were added to the model: age, gender, season of blood collection, use of NSAIDs, BMI, hours of moderate to vigorous physical activity and stage of disease at diagnosis [7, 17, 19, 31]. In addition, having comorbidities at diagnosis (yes/no) changed the regression coefficient substantially (>10\%) and was therefore added to the model as well. All models were adjusted for cohort. The use of statins, smoking status and dietary factors, such as vitamin D, calcium, magnesium, dietary fat, alcohol and fibre, did not influence the regression coefficient and were thus not taken into account in the models. 
Mixed model regression was used to determine the overall mean association between $25(\mathrm{OH}) \mathrm{D}_{3}$ levels and inflammatory markers over time. Linear mixed models take into account both the individual changes in serum vitamin D levels (random effects) and the average change in the population (fixed effects) by using all available measurements and including patients with incomplete data [32]. Time was added as a continuous variable. As fixed effects, we included cohort, season of blood collection, age, gender, use of NSAIDs, BMI, physical activity, having comorbidities, stage of disease and physical activity * time. As random effects we included subject. The unstructured co-variance model was used. Inter- and intra-individual associations were disaggregated by adding centered personmean values to the model to estimate inter-individual associations and individual deviations from the person-mean value to estimate intra-individual associations [33]. It is important to disaggregate intra (within) and inter (between) person associations, since results obtained from group-level data cannot always be directly translated to individuals [33].

Stratified analyses were done for stage of disease (I, II, III, IV), gender, and cohort (COLON, EnCoRe). A sensitivity analysis was done including only those patients with hsCRP values < $10 \mu \mathrm{g} / \mathrm{ml}$, thus excluding those with acute inflammation. Also a sensitivity analyses was done excluding inflammatory markers measured at diagnosis when studying the overall mean associations, since inflammatory markers measured at time of diagnosis may be influenced by the procedure performed to make the diagnosis.

To interpret the beta coefficient of the regression line, the exponential of the beta was taken $\left(E X P^{\wedge} \beta\right)$, since the outcome variable was natural log transformed. These interpreted betas (expressed in percentages) are reported in the text in the results section. In addition, all results described in the result sections are derived from the adjusted models/model 2.

Statistical analyses were performed in SAS 9.4 (SAS Institute, Cary NC). P-values $<0.05$ were considered statistically significant.

\section{RESULTS}

\section{Patient' Characteristics}

We included 798 CRC patients of whom 252 (32\%) were female (Table 1). Median age was 66.9 [IQR 62.2-73.0] years. Two-thirds of the patients had colon cancer. At presentation, only $7 \%$ of the patients were in stage IV of disease, $28 \%$ presented with stage I of disease, $26 \%$ with stage II, and $39 \%$ with stage III.

Patient characteristics for the total population and stratified by vitamin $D$ status are shown in Table 1. Patients who had sufficient ( $\geq 50 \mathrm{nmol} / \mathrm{L}$ ) vitamin D levels $(n=448)$ were 
more often female, were more often diagnosed with stage I disease, used more frequent NSAIDs, used more frequent vitamin D supplements and were more physically active compared to patients who had insufficient levels.

Table 1 Baseline characteristics of colorectal cancer patients stratified by vitamin D status

\begin{tabular}{|c|c|c|c|}
\hline & $\begin{array}{l}\text { Total population } \\
\qquad \mathbf{N}=798\end{array}$ & $\begin{array}{l}\text { Vitamin D insufficient } \\
\qquad \begin{array}{c}(<50 \mathrm{nmol} / \mathrm{L}) \\
\mathrm{N}=333\end{array}\end{array}$ & $\begin{array}{l}\text { Vitamin D sufficient } \\
\begin{array}{c}\text { ( } \geq 50 \mathrm{nmol} / \mathrm{L}) \\
\mathrm{N}=448\end{array}\end{array}$ \\
\hline Age (years) & $66.9[62.2-73.0]$ & $66.9[61.8-73.8]$ & $67.0[62.8-72.5]$ \\
\hline Gender (female) & $252(32)$ & $91(27)$ & $155(35)$ \\
\hline \multicolumn{4}{|l|}{ Education level $^{a}$} \\
\hline Low & $313(41)$ & $123(38)$ & $183(42)$ \\
\hline Medium & $243(32)$ & $103(32)$ & $136(32)$ \\
\hline High & $210(27)$ & $95(30)$ & $112(26)$ \\
\hline Unknown & 32 & 12 & 17 \\
\hline \multicolumn{4}{|l|}{ Season of blood collection ${ }^{b}$} \\
\hline Spring & $223(29)$ & 129 (39) & $94(21)$ \\
\hline Summer & $227(29)$ & $46(14)$ & $181(41)$ \\
\hline Autumn & $140(18)$ & $50(15)$ & $90(20)$ \\
\hline Winter & $185(24)$ & $106(32)$ & $79(18)$ \\
\hline Unknown & 23 & 2 & 4 \\
\hline Serum $25(\mathrm{OH}) \mathrm{D}_{3}(\mathrm{nmol} / \mathrm{l})$ & $53.9[40.8-70.2]$ & $38.5[28.9-44.5]$ & $67.2[58.1-79.8]$ \\
\hline Unknown & 17 & & \\
\hline Interleukin 10 (pg/mL) & $0.3[0.2-0.4]$ & $0.3[0.2-0.4]$ & $0.2[0.2-0.4]$ \\
\hline Unknown & 67 & 24 & 28 \\
\hline Interleukin 8 (pg/mL) & $5.8[4.3-8.2]$ & $5.9[4.4-8.5]$ & $5.7[4.2-8.1]$ \\
\hline Unknown & 29 & 5 & 9 \\
\hline Interleukin 6 (pg/mL) & $1.0[0.7-1.7]$ & $1.1[0.8-1.8]$ & $1.0[0.7-1.6]$ \\
\hline Unknown & 31 & 5 & 11 \\
\hline $\begin{array}{l}\text { Tumour necrosis factor } \alpha \\
(\mathrm{pg} / \mathrm{mL})\end{array}$ & $2.1[1.7-2.6]$ & $2.1[1.7-2.6]$ & $2.0[1.6-2.6]$ \\
\hline Unknown & 29 & 6 & 8 \\
\hline C-reactive protein $(\mu \mathrm{g} / \mathrm{ml})$ & $2.5[1.1-6.0]$ & $2.9[1.2-6.9]$ & $2.4[1.1-5.3]$ \\
\hline Unknown & 112 & 40 & 56 \\
\hline \multicolumn{4}{|l|}{ Type of Cancer } \\
\hline Colon & $512(64)$ & $204(61)$ & $298(67)$ \\
\hline Rectal & $286(36)$ & $129(39)$ & $150(33)$ \\
\hline \multicolumn{4}{|l|}{ Tumour stage } \\
\hline I & $209(28)$ & $77(24)$ & $132(31)$ \\
\hline II & $200(26)$ & $84(26)$ & $116(27)$ \\
\hline III & $293(39)$ & $139(43)$ & $153(36)$ \\
\hline IV & $52(7)$ & $21(7)$ & $30(7)$ \\
\hline Unknown & 44 & 12 & 17 \\
\hline \multicolumn{4}{|l|}{ Type of treatment } \\
\hline Surgery only & $419(54)$ & $167(52)$ & $247(58)$ \\
\hline Surgery + chemotherapy & $171(22)$ & $68(21)$ & $97(23)$ \\
\hline Surgery + radiotherapy & $97(13)$ & $53(17)$ & $41(10)$ \\
\hline Surgery + chemo radiation & $69(9)$ & $30(10)$ & $37(9)$ \\
\hline
\end{tabular}




\begin{tabular}{|c|c|c|c|}
\hline Unknown & 34 & 11 & 19 \\
\hline Comorbidities (yes) & $592(74)$ & $248(74)$ & $331(74)$ \\
\hline Daily use of NSAIDs (yes) & $157(20)$ & $58(17)$ & $98(22)$ \\
\hline BMI $\left(\mathrm{kg} / \mathrm{m}^{2}\right)$ & 26.5 [24.4-29.5] & 26.9 [24.5-30.6] & $26.2[24.3-29.1]$ \\
\hline Unknown & 5 & 2 & 3 \\
\hline \multicolumn{4}{|l|}{ Smoking } \\
\hline Current & $98(13)$ & $39(12)$ & $57(13)$ \\
\hline Former & $463(59)$ & $193(59)$ & $263(60)$ \\
\hline Never & $221(28)$ & $97(29)$ & $117(27)$ \\
\hline Unknown & 16 & 4 & 11 \\
\hline Moderate to vigorous & $11.5[5.0-20.0]$ & $10.0[4.3-19.0]$ & $12.8[6.0-21.2]$ \\
\hline \multicolumn{4}{|l|}{ physical activity } \\
\hline (hours/week) $^{c}$ & 18 & 4 & 12 \\
\hline \multicolumn{4}{|l|}{ Unknown } \\
\hline \multicolumn{4}{|l|}{ Dietary intake } \\
\hline Dietary vitamin $D(\mu \mathrm{g} /$ day) & $3.2[2.2-4.2]$ & $3.1[2.2-4.2]$ & $3.2[2.2-4.3]$ \\
\hline Total vitamin $\mathrm{D}(\mu \mathrm{g} / \mathrm{day})^{\mathrm{d}}$ & $3.8[2.6-6.2]$ & $3.4[2.4-4.6]$ & $4.3[2.9-8.5]$ \\
\hline Calcium (mg/day) & $873[655-1101]$ & $880[651-1116]$ & 871 [656-1089] \\
\hline Fibre (g/day) & 21.4 [16.8-26.5] & 20.9 [16.6-26.5] & $21.6[17.2-26.3]$ \\
\hline Total fat (g/day) & $71.3[55.1-91.9]$ & $71.6[54.3-92.1]$ & $70.8[55.4-90.2]$ \\
\hline EPA (g/day) & $0.06[0.03-0.10]$ & $0.05[0.03-0.10]$ & $0.07[0.04-0.12]$ \\
\hline DHA (g/day) & $0.08[0.04-0.15]$ & $0.07[0.03-0.13]$ & $0.08[0.04-0.16]$ \\
\hline Unknown & 22 & 7 & 14 \\
\hline \multicolumn{4}{|l|}{ Use of vitamin D } \\
\hline supplements (yes) & $210(26)$ & $50(15)$ & $160(36)$ \\
\hline Unknown & 12 & 3 & 9 \\
\hline
\end{tabular}

Values presented are median [quartile 1 - quartile 3] or number (percentage). BMI: Body Mass Index, EPA: eicosapentaenoic acid, DHA: docosahexaenoic acid, NSAIDs: non-steroidal anti-inflammatory drugs.

a: Low education was defined as primary school and lower general secondary education; medium as lower vocational training and higher general secondary education; high as high vocational training and university. b: spring: March-May; summer: June-August; autumn: September-November; winter: December-February. c: Activities with a Metabolic Equivalent score (MET score) $\geq 3$ were defined as moderate to vigorous physical activity

$\mathrm{d}$ : total vitamin D intake from diet and supplements.

\section{CiRCULATING LEVELS OF 25(OH)D $D_{3}$ AND INFLAMMATORY MARKERS}

Blood levels of $25(\mathrm{OH}) \mathrm{D}_{3}$ were higher two years after diagnosis $(64, \mathrm{IQR} 49-83 \mathrm{nmol} / \mathrm{L})$ compared to levels at diagnosis (54, IQR $41-70 \mathrm{nmol} / \mathrm{L}$ ) (Table 2). Levels of IL10, IL8, IL6 and TNF $\alpha$, did not substantially change over time. Levels of hsCRP were slightly lower one year after diagnosis (1.7 mg/L, IQR 0.7-4.4) compared to levels at diagnosis $(2.5 \mathrm{mg} / \mathrm{L}$, IQR 1.1-6.0). Comparable results were found when only patients were included who donated blood at three or more time points (Supplementary data Table S1). 


\section{ASSOCIATIONS BETWEEN 25(OH) D 3 LEVELS AND INFLAMMATORY MARKERS}

A $10 \mathrm{nmol} / \mathrm{L}$ higher $25(\mathrm{OH}) \mathrm{D}_{3}$ was associated with a $6.8 \%(95 \% \mathrm{Cl}-8.7 ;-3.9)$ lower IL6 level at diagnosis, a $4.9 \%(95 \% \mathrm{Cl}-7.0 ;-2.0)$ lower IL6 level six months after diagnosis and a $5.8 \%$ $(95 \% \mathrm{Cl}-12.2 ;-0.1)$ lower IL6 level one year after diagnosis (Table 3). A statistically nonsignificant association between $25(\mathrm{OH}) \mathrm{D}_{3}$ and IL6 was found two years after diagnosis ($3.0 \%, 95 \% \mathrm{Cl}-6.8 ; 0,0)$. No associations were observed between $25(\mathrm{OH}) \mathrm{D}_{3}$ and IL10, IL8, TNF- $\alpha$ and hsCRP at the separate time points. At diagnosis, but not at follow-up time points, a statistically significant inverse association was found between $25(\mathrm{OH}) \mathrm{D}_{3}$ and the summary inflammatory $z$-score $(\beta-14.0 \%, 95 \% \mathrm{Cl}-25.2 ;-2.0)$.

When combining all data using mixed models, statistically significant inverse associations between $25(\mathrm{OH}) \mathrm{D}_{3}$ and IL6, IL10 and the summary inflammatory z-score were observed (Table 4). A $10 \mathrm{nmol} / \mathrm{L}$ higher 25(OH) $\mathrm{D}_{3}$ level was associated with a $5.8 \%(95 \% \mathrm{Cl}-7.7 ;-3.9)$ lower IL6 level, a $2.0 \%(95 \% \mathrm{Cl}-3.9 ;-0.1)$ lower IL10 level and a $15.6 \%(95 \% \mathrm{Cl}-21.1 ;-7.7)$ lower inflammatory summary z-score.

When investigating the association within and between individuals, we found stronger associations within individuals compared to between individuals for IL10, IL6, TNF- $\alpha$ and the inflammatory z-score. A significant association between $25(\mathrm{OH}) \mathrm{D}_{3}$ levels and TNF $\alpha$ was found within individuals $(\beta-0.0295 \% \mathrm{Cl}-0.03 ;-0.00)$ but not between individuals $(\beta 0.00$ $95 \% \mathrm{Cl}-0.01 ; 0.01)$. Also, an association between $25(\mathrm{OH}) \mathrm{D}_{3}$ levels and IL10 was found within individuals $(\beta-0.0495 \% \mathrm{Cl}-0.06 ;-0.01)$ but not between individuals $(\beta 0.0095 \% \mathrm{Cl}$ $0.02 ; 0.03)$.

\section{$\underline{\text { Stratified analyses }}$}

Stratified analyses for stage of disease showed slightly stronger associations between $25(\mathrm{OH}) \mathrm{D}_{3}$ and IL6 in stage II $(-3.9 \% 95 \% \mathrm{Cl}-7.7 ; 1.0)$, III $(-5.8 \% 95 \% \mathrm{Cl}-8.6 ;-3.9)$ and IV ($4.9 \% 95 \% \mathrm{Cl}-11.3 ; 1.0)$ compared to stage I $(-2.0 \% 95 \% \mathrm{Cl}-4.9 ; 0.1)$. Comparable results were observed for the summary z-score (table 5 ). No differences between men and women were observed (Table 5). Finally, similar results were found in the COLON study and the EnCoRe study regarding IL10, IL8, IL6 and TNF $\alpha$ (table 5). However, an association between $25(\mathrm{OH}) \mathrm{D}_{3}$ and hsCRP was found in the EnCoRe study $(-6.8 \% 95 \% \mathrm{Cl}-11.3 ;-2.0)$ but not in the COLON study $(-0.1 \% 95 \% \mathrm{Cl}-3.9 ; 3.0)$. Consequently, the association between $25(\mathrm{OH}) \mathrm{D}_{3}$ and the summary inflammatory z-score was stronger in the EnCoRe study ($24.4 \% 95 \% \mathrm{Cl}-34.4 ;-13.1)$ compared to the COLON study $(-10.4 \% 95 \% \mathrm{Cl}-18.1 ;-2.0)$. Similar associations were observed when excluding patients with hsCRP levels $>10 \mu \mathrm{g} / \mathrm{mL}$ and when excluding baseline measurements. 


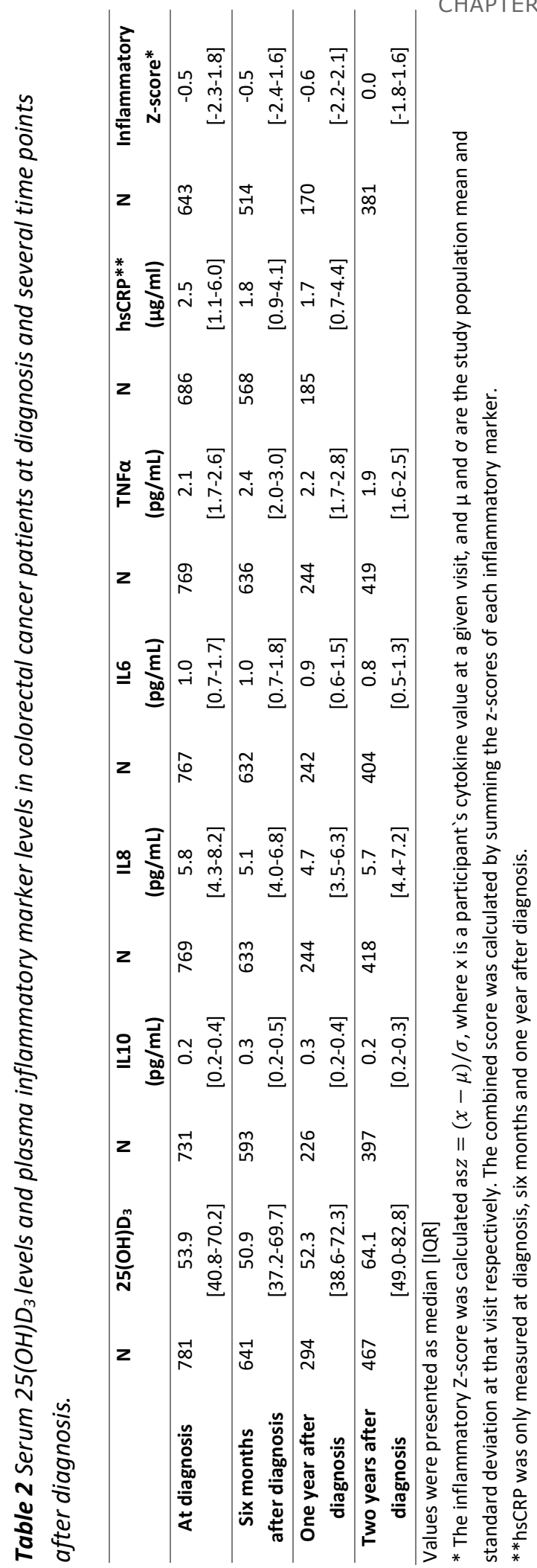


CIRCULATING LEVELS OF 25(OH)D3 AND INFLAMMATION MARKERS

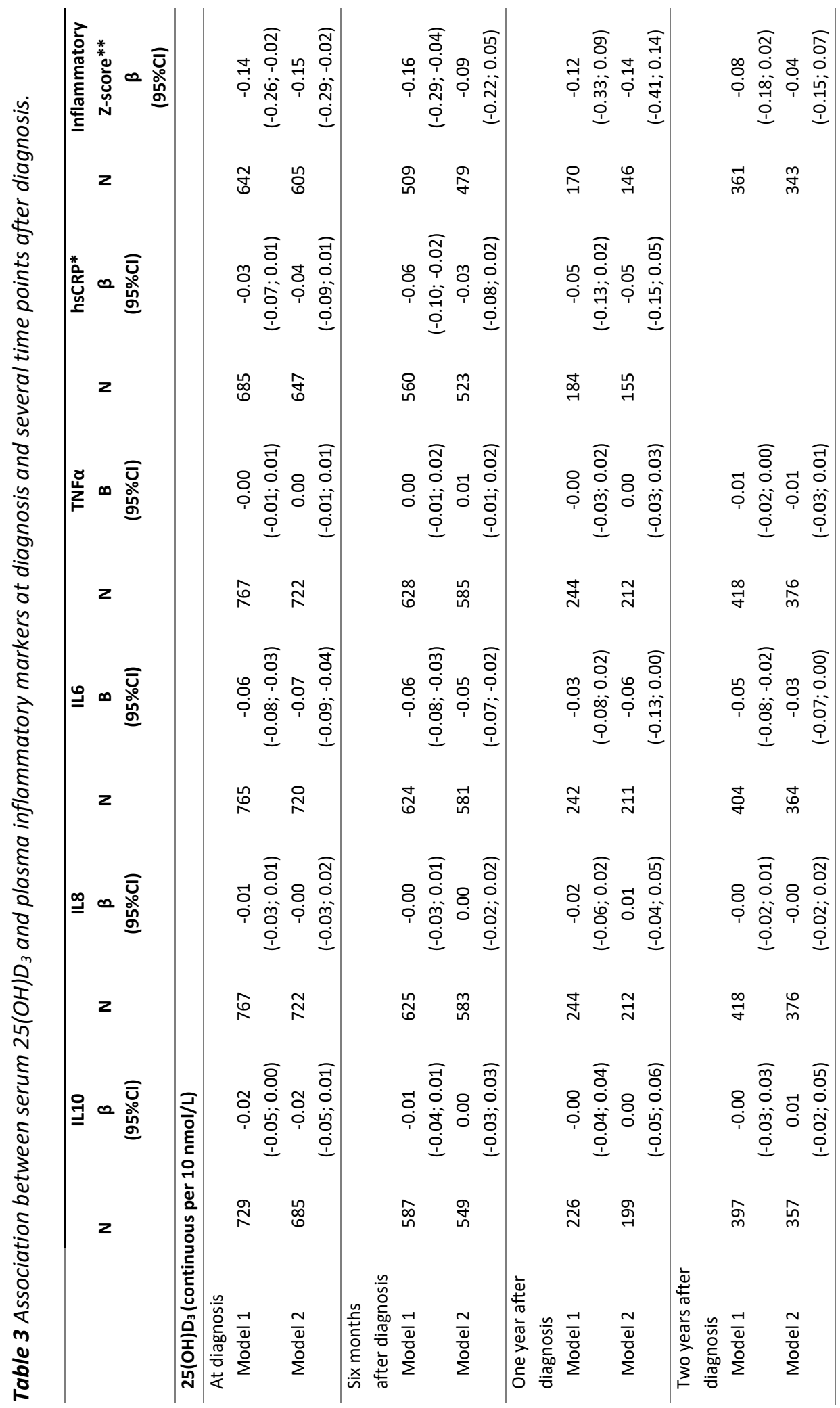




\section{CHAPTER 5}

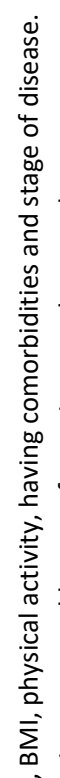

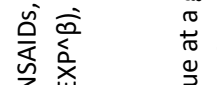

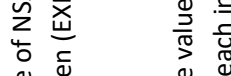

崩离

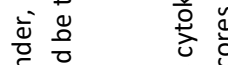

离 $\frac{0}{3}$

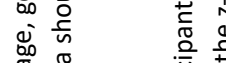

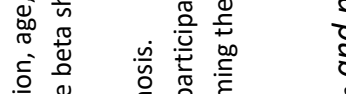

每先

类

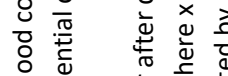

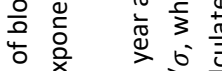

ठ

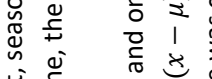

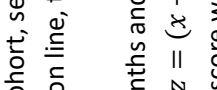

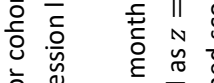

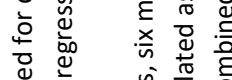

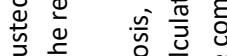

龸

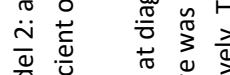

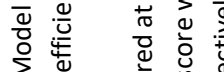

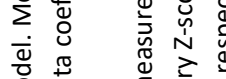

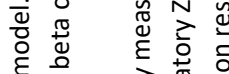

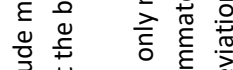

茯

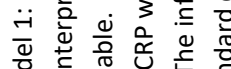

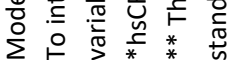

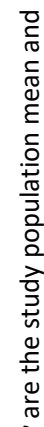

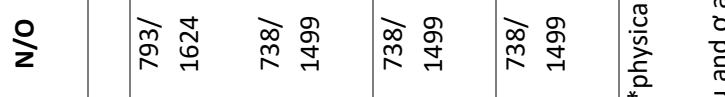

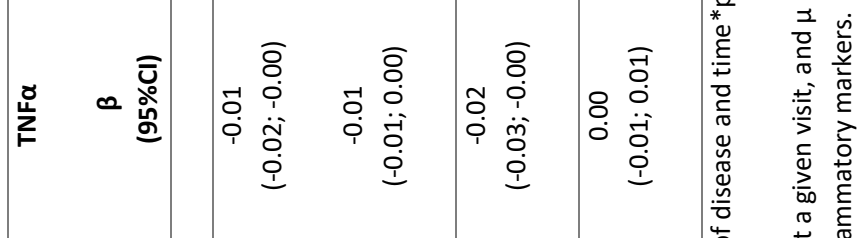

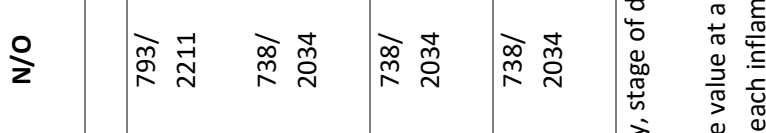

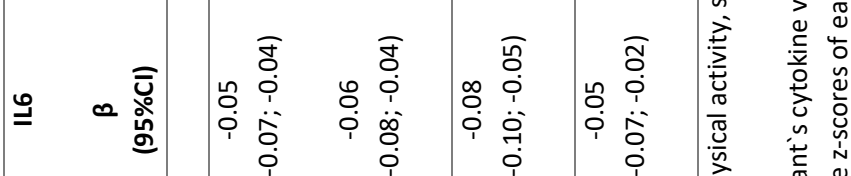

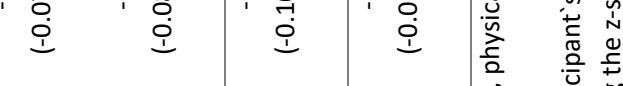

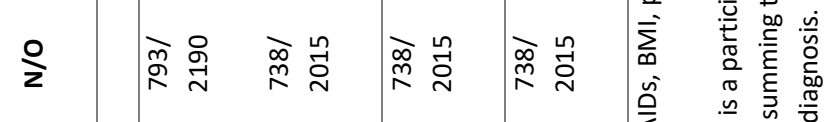

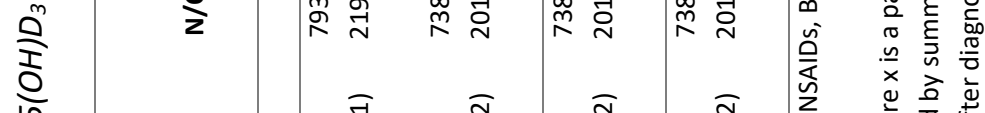

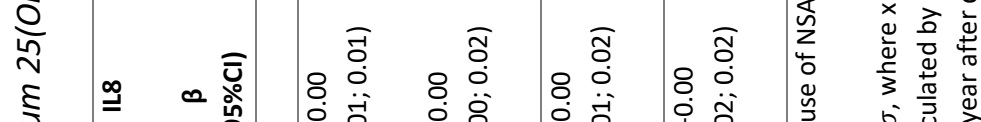

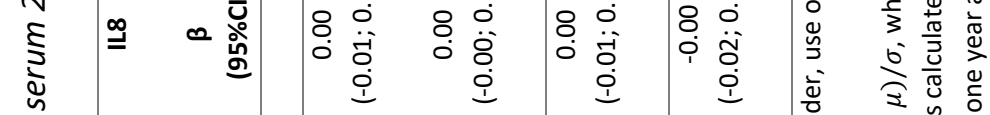

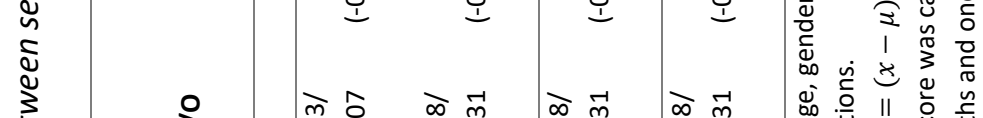

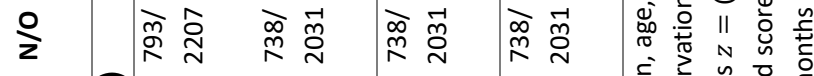
Ј

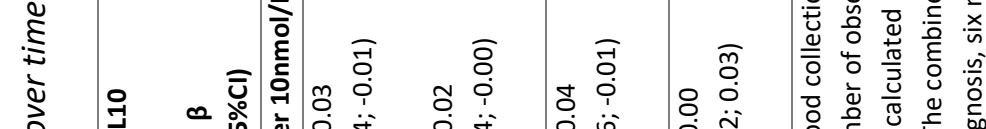

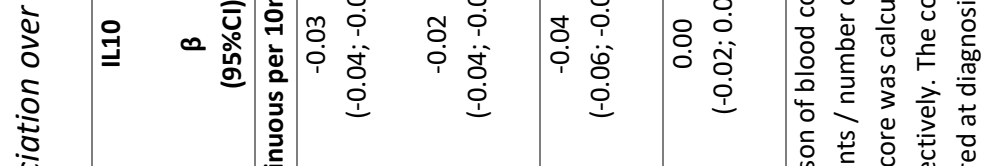
z *

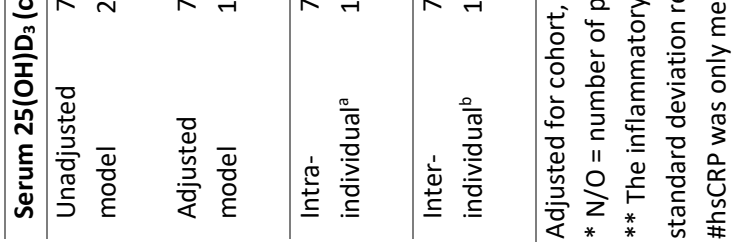




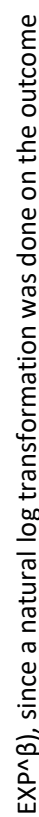

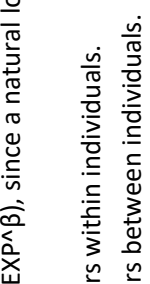

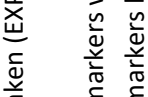

$\stackrel{0}{\circ}$

$\frac{0}{\frac{0}{\partial}}$

要

$\stackrel{0}{\underline{1}}$

莙$$
\text { 茫 }
$$

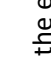

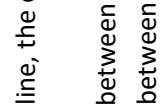

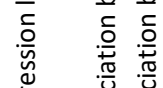

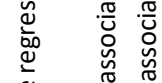

$\stackrel{2}{\Perp} \quad \stackrel{0}{ \pm}$

施

$\stackrel{+\frac{U}{U}}{\frac{U}{9}}$

迹

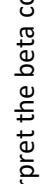

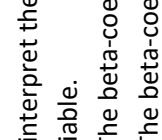

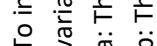
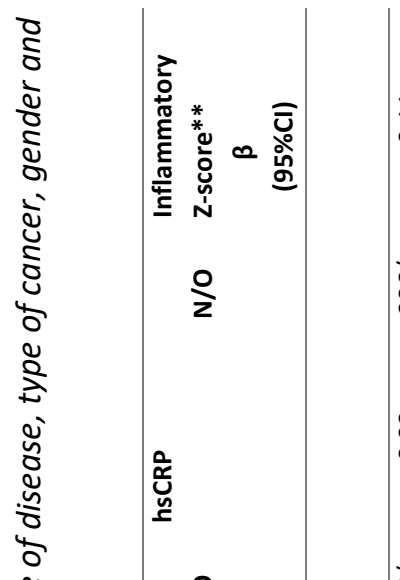

产

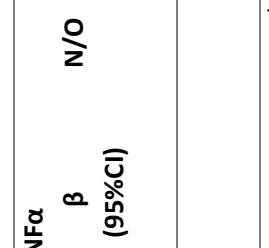

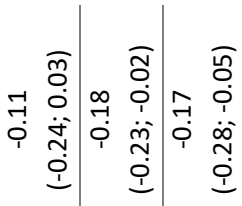

ఫ্

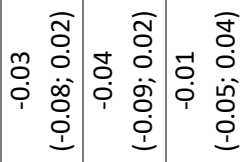

ঠ্ণ

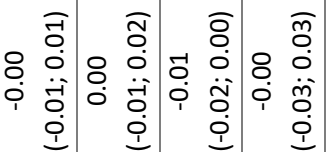

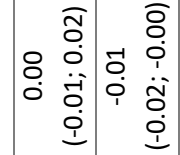

¿্ํ

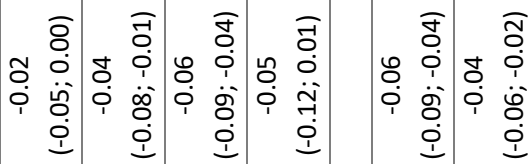

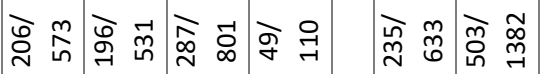

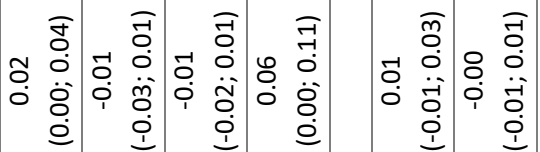

$\stackrel{\infty}{=}$

๑ ํํํ

$\stackrel{m}{\text { I }}$

กั

ई

$\frac{\sqrt{2}}{\mathfrak{2}}$

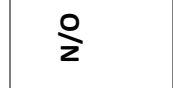

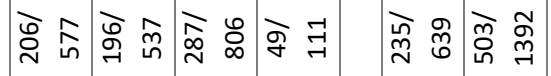

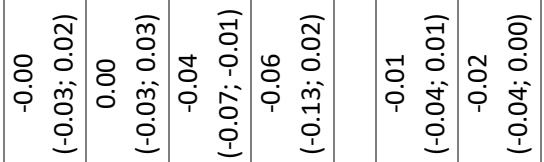

을 ڤั

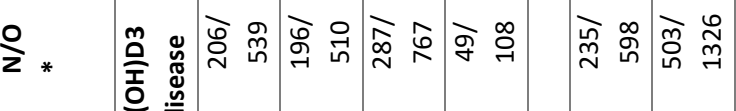




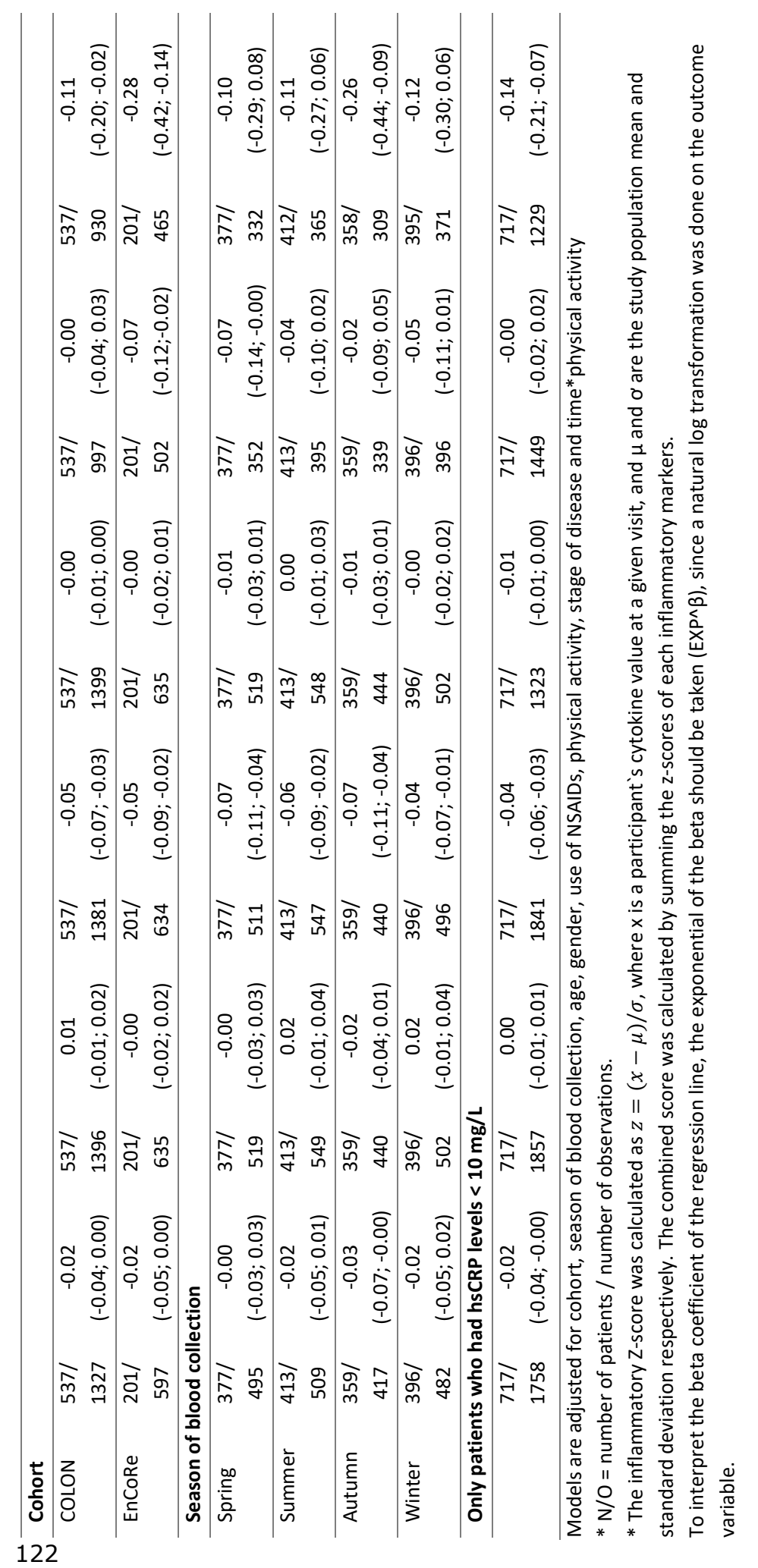




\section{DISCUSSION}

Circulating levels of pro-inflammatory cytokines were generally low at diagnosis and during follow up in prospectively analysed CRC patients. A statistically significantly inverse association between $25(\mathrm{OH}) \mathrm{D}_{3}$ levels and IL6 levels was observed at diagnosis, six months and one year after diagnosis and when combining all time points. At diagnosis and when combining all time points also a significantly inverse association between $25(\mathrm{OH}) \mathrm{D}_{3}$ and the summary inflammatory z-score was observed.

Plasma levels of cytokines were generally low in CRC patients. We found a median level of $1.0 \mathrm{pg} / \mathrm{ml}$ for IL6, $5.8 \mathrm{pg} / \mathrm{ml}$ for IL8, $0.2 \mathrm{\rho g} / \mathrm{ml}$ for IL10 and $2.0 \mathrm{\rho g} / \mathrm{ml}$ for TNF $\alpha$ at diagnosis. Other studies measuring inflammatory markers in CRC patients found higher levels, with median IL6 levels ranging between 2.8 and $35.7 \mathrm{\rho g} / \mathrm{ml}$ [34-38], median IL8 levels ranging between 25 and $114 \mathrm{\rho g} / \mathrm{ml}[35,38,39]$, median IL10 levels ranging between 7 and 24 $\rho \mathrm{g} / \mathrm{ml}[37,38,40]$ and median TNF $\alpha$ levels ranging between 16 and $272 \mathrm{\rho g} / \mathrm{ml}[35,38]$. However, the study of Hopkins et al., found levels comparable to ours in colorectal adenoma patients (median IL6 level $1.1 \mathrm{\rho g} / \mathrm{ml}$, median IL8 level $5.5 \mathrm{\rho g} / \mathrm{ml}$, median IL10 level $0.5 \mathrm{\rho g} / \mathrm{ml}$ and median TNF level $3.4 \mathrm{\rho g} / \mathrm{ml}$ ) [17]. Differences between levels of inflammatory markers could be explained by the methods used to assess levels of inflammatory markers. The study of Hopkins and colleagues used a comparable method, namely high sensitivity multiplex ELISA, while other studies measured inflammatory markers using a method based on flow cytometry or sandwich ELISA. Furthermore, Hopkins et al., measured inflammatory markers in plasma, as we did, while the other studies mentioned above measured inflammatory markers in serum. A recent study concluded that plasma is a more sensitive matrix for detecting changes in low levels of cytokines [41]. Furthermore, that study found a higher non-specific background in serum compared to plasma cytokines [41]. The different biological specimen (plasma vs serum) and laboratory methods that are used make it hard to compare absolute values. To improve comparability of different studies, method harmonisation is required.

In the present study, we observed a statistically significant inverse association between $25(\mathrm{OH}) \mathrm{D}_{3}$ levels and IL6 levels. To the best of our knowledge, no other studies assessed the association between $25(\mathrm{OH}) \mathrm{D}_{3}$ and IL6 levels and other inflammatory markers in CRC patients. A study in colorectal adenoma patients $(n=92)$ found a non-significant reduction in IL6 levels after supplementation with vitamin D3 [17]. Another study in obese individuals $(n=147)$ observed an inverse association between serum $25(\mathrm{OH}) \mathrm{D}_{3}$ and IL6 levels [19]. The association between $25(\mathrm{OH}) \mathrm{D}_{3}$ levels and IL6 is of potential interest as IL6 has an important role in chronic inflammation [42] and is also suggested to stimulate cancer progression [22, 34, 43]. IL6 is important in the transmission from beneficial acute inflammation to harmful chronic inflammation [42]. It is involved in the recruitment of macrophages into the tissue leading to chronic inflammation proliferation [42] and 
probably increases tumorigenesis. Besides, IL6 is also know to stimulate STAT3, which is an oncogene [22]. Stimulation of STAT3 promotes tumour growth by facilitating cell proliferation and inhibition of apoptosis [22]. Finally, higher levels of IL6 were associated with increased expression of matrix metalloproteases favouring tumour escape from apoptosis and metastasis $[44,45]$. Lowering IL6 levels could thus possibly improve CRC prognosis.

Apart from an association between $25(\mathrm{OH}) \mathrm{D}_{3}$ and IL6, we did not find associations between $25(\mathrm{OH}) \mathrm{D}_{3}$ and the other inflammatory markers. A possible explanation for not finding a significant association here is the central role of IL6, compared to the other inflammatory markers in chronic inflammation $[5,42,46]$. However, when inter- and intraindividual associations were disaggregated, intra-individual analyses also showed a significant association between $25(\mathrm{OH}) \mathrm{D}_{3}$ and TNF $\alpha$ and IL10. Thus, within individuals an increase in $25(\mathrm{OH}) \mathrm{D}_{3}$ levels was associated with a decrease in IL6, TNF $\alpha$ and IL10 levels. This indicates that within individuals an increase in $25(\mathrm{OH}) \mathrm{D}_{3}$ levels, due to either supplementation or sunlight exposure, may lead to a lower systemic inflammatory status.

This study also showed an inverse association between $25(\mathrm{OH}) \mathrm{D}_{3}$ and the summary inflammatory z-score. In line with our findings a study in colorectal adenoma patients ( $n=92$ ) also found a statistically significant lower inflammatory z-score after supplementation with vitamin D3 [17]. In both studies a summary inflammatory z-score was used assuming equal contribution of each inflammatory marker. However, the role of cytokines in the progression of cancer is complex [22], as several cytokines probably act in synergy [47] or antagonistically. In addition, it could be that some cytokines are more important in the progression of cancer. Thus, although knowledge is currently lacking, adding a weighting factor for each cytokine might be more appropriate.

Although we found an inverse association between vitamin D levels and levels of inflammatory markers, the associations were relatively small, as a $10 \mathrm{nmol} / \mathrm{L}$ higher $25(\mathrm{OH}) \mathrm{D}_{3}$ level was associated with a $6 \%$ lower IL6 levels and a $16 \%$ lower summary zscore. Previous studies showed that inflammatory cytokines are associated with quality of life and cancer recurrence and survival [8]. Lowering the inflammatory status is a promising way to control cancer. However, the question remains whether this can be achieved by increasing $25(\mathrm{OH}) \mathrm{D}_{3}$ levels. To further investigate this, a well powered intervention study focussing on the effects of increasing vitamin D levels on inflammation would be needed. Above that, further studies should elucidate underlying mechanisms involved in inflammation and the progression of CRC and the role of vitamin $D$ in this. One way to examine this is by investigating differences in expression of genes involved in inflammatory processes in CRC tumour tissues in patients with high vitamin D levels compared to patients with low vitamin D levels. 
The present study has some limitations. First of all, plasma levels of inflammatory markers, especially cytokines, were relatively low. This could limit the ability to detect associations. However, we found associations between $25(\mathrm{OH}) \mathrm{D}_{3}$ and IL6 levels as well as between $25(\mathrm{OH}) \mathrm{D}_{3}$ levels and the summary inflammatory z-score. Secondly, since vitamin $D$ and inflammatory markers were measured at the same time points, we cannot conclude from this study whether vitamin D decreases inflammatory markers or the other way around. However, results of previous studies, in other populations, showed a decrease in inflammatory markers after vitamin D supplementation $[17,18]$. In addition, the antiinflammatory effects of vitamin D are well studied [11, 21, 48].

The present study also has some important strengths. First of all, this prospective study measured serum $25(\mathrm{OH}) \mathrm{D}_{3}$ and a set of inflammatory markers simultaneously before and after treatment in CRC patients. Another strength is the use of a multiplex assay, allowing to measure several cytokines at once in a single small plasma sample. Above that, our multiplex assay with chemiluminescence detection has very low detection limits, which is essential since many cytokines exist in very low levels in the peripheral blood. It should be mentioned that testing several inflammation markers ( 5 in total) raises the risk of false positive findings. However, we are confident that the associations observed between $25(\mathrm{OH}) \mathrm{D}_{3}$ and IL6 are not chance findings. As we found these associations very consistently throughout our study, at several time points (at diagnosis and during followup), in both cohorts and in almost all strata's of the stratified analyses. Furthermore, in order to exclude pre-analytical artefacts due to e.g. degradation, we eliminated samples collected more than two years before, since several cytokines including IL6 and IL10 degrade after 2 years of storage at -80 degrees Celsius [26]. Finally, due the availability of detailed data on diet and other clinical and lifestyle factors, we could adjust for the most plausible confounders, although residual confounding can never be fully excluded.

To conclude, serum $25(\mathrm{OH}) \mathrm{D}_{3}$ levels were inversely associated with plasma IL6 levels and a summary inflammatory z-score in CRC patients at different time points before and after treatment. Further intervention studies, investigating the effect of increasing vitamin $D$ levels on inflammatory mediators in CRC patients are needed. 


\section{ADDITIONAL INFORMATION}

\section{FUNDING}

Wereld Kanker Onderzoek Fonds, including funds from grant 2014/1179 as part of the World Cancer Research Fund International Regular Grant Programme; Alpe d'Huzes/Dutch Cancer Society (UM 2012-5653, UW 2013-5927, UW 2015-7946); and ERA-NET on Translational Cancer Research (TRANSCAN/Dutch Cancer Society (UW2013-6397, UW2014-6877) and the Netherlands Organization for Health Research and Development (ZonMw, the Netherlands). The EnCoRe study was supported by a grant from the Stichting Alpe d'HuZes within the research program 'Leven met kanker' of the Dutch Cancer Society (Grant No. UM-2010-4867) and by a grant from Kankeronderzoekfonds Limburg as part of Health Foundation Limburg (Grant No. 00005739).

\section{AUTHORS' CONTRIBUTIONS}

E Wesselink, MJL Bours, HJW de Wilt, RF Witkamp, DE Kok, MP Weijenberg, E Kampman and FJB van Duijnhoven contributed to the design and the conceptualization of this study. E Wesselink, HJW de Wilt, H van Baar, AJMR Geijsen, B Hansson, ETP Keulen, J van den Ouweland and $\mathrm{M}$ van Zutphen contributed to recruitment of participants and the data collection. Statistical data analyses were done by E Wesselink. The manuscript was drafted by $E$ Wesselink and FJB van Duijnhoven, and all authors critically read and revised the manuscript. All authors approved the final version of the manuscript.

\section{ACKNOWLEDGEMENTS}

The authors would like to thank the participants of the COLON study and the investigators at Wageningen University \& Research and the co-workers from the following hospitals for their involvement in recruitment for the COLON study: Hospital Gelderse Vallei, Ede; Radboudumc, Nijmegen; Slingeland Hospital, Doetinchem; Canisius Wilhelmina Hospital, Nijmegen; Rijnstate Hospital, Arnhem; Gelre Hospitals, Apeldoorn/Zutphen; Hospital Bernhoven, Uden; Isala, Zwolle; ZGT, Almelo; Martini Hospital, Groningen; Admiraal de Ruyter Hospital, Goes/Vlissingen. We would like to thank all participants of the EnCoRe study and the health professionals in the three hospitals involved in the recruitment of participants of the study: Maastricht University Medical Center, VieCuri Medical Center, and Zuyderland Medical Center. We would also like to thank the MEMIC center for data and information management for facilitating the logistic processes and data management of our study. Furthermore, we would like to thank the research dieticians and research assistant who are responsible for patient inclusion and follow-up, performing home visits, as well as data collection and processing. Finally, the authors would like to thank BEVITAL, Bergen, Norway for analyzing hsCRP in the COLON and EnCoRe study. 


\section{AVAILABILITY OF DATA AND MATERIAL}

Since the data consist of identifying cohort information, some access restrictions apply and therefore cannot be made publicly available. Data will be shared with permission from the acting committee of the COLON Study. Requests for data can be sent to Dr. Fränzel van Duijnhoven, Division of Human Nutrition and Health, Wageningen University \& Research, The Netherlands.

Email: franzel.vanduijnhoven@wur.nl. 


\section{REFERENCES}

[1] Hanahan D, Weinberg RA. Hallmarks of cancer: the next generation. Cell. 2011;144:646-74.

[2] Aggarwal BB, Vijayalekshmi R, Sung B. Targeting inflammatory pathways for prevention and therapy of cancer: short-term friend, long-term foe. Clinical Cancer Research. 2009;15:425-30.

[3] Grivennikov SI, Greten FR, Karin M. Immunity, inflammation, and cancer. Cell. 2010;140:883-99.

[4] Shalapour S, Karin M. Immunity, inflammation, and cancer: an eternal fight between good and evil. The Journal of clinical investigation. 2015;125:3347-55.

[5] Lasry A, Zinger A, Ben-Neriah Y. Inflammatory networks underlying colorectal cancer. Nature immunology. 2016;17:230.

[6] Chang PH, Pan YP, Fan CW, Tseng WK, Huang JS, Wu TH, et al. Pretreatment serum interleukin-1 6 , and tumor necrosis factor- $\alpha$ levels predict the progression of colorectal cancer. Cancer medicine. 2016;5:42633.

[7] Rasic I, Radovic S, Aksamija G. Relationship between chronic inflammation and the stage and histopathological size of colorectal carcinoma. Medical Archives. 2016;70:104.

[8] Seruga B, Zhang H, Bernstein L, Tannock IF. Cytokines and their relationship to the symptoms and outcome of cancer. Nature Reviews Cancer. 2008;8:887.

[9] Xu J, Ye Y, Zhang H, Szmitkowski M, Mäkinen M, Li P, et al. Diagnostic and prognostic value of serum interleukin-6 in colorectal cancer. Medicine. 2016;95.

[10] Coyle C, Cafferty FH, Langley RE. Aspirin and colorectal cancer prevention and treatment: is it for everyone? Current colorectal cancer reports. 2016;12:27-34.

[11] Krishnan AV, Feldman D. Mechanisms of the anti-cancer and anti-inflammatory actions of vitamin D. Annual review of pharmacology and toxicology. 2011;51:311-36.

[12] Terzić J, Grivennikov S, Karin E, Karin M. Inflammation and colon cancer. Gastroenterology. 2010;138:210114. e5.

[13] Bikle DD. Vitamin D metabolism, mechanism of action, and clinical applications. Chemistry \& biology. 2014;21:319-29.

[14] Aranow C. Vitamin D and the immune system. Journal of investigative medicine. 2011;59:881-6.

[15] Karin M, Lin A. NF-KB at the crossroads of life and death. Nature immunology. 2002;3:221.

[16] Wang S, Liu Z, Wang L, Zhang X. NF-KB signaling pathway, inflammation and colorectal cancer. Cellular \& molecular immunology. 2009;6:327.

[17] Hopkins MH, Owen J, Ahearn T, Fedirko V, Flanders WD, Jones DP, et al. Effects of supplemental vitamin D and calcium on biomarkers of inflammation in colorectal adenoma patients: a randomized, controlled clinical trial. Cancer prevention research. 2011;4:1645-54.

[18] Chandler PD, Scott JB, Drake BF, Ng K, Manson JE, Rifai N, et al. Impact of vitamin D supplementation on inflammatory markers in African Americans: results of a four-arm, randomized, placebo-controlled trial. Cancer prevention research. 2014;7:218-25.

[19] Bellia A, Garcovich C, D’Adamo M, Lombardo M, Tesauro M, Donadel G, et al. Serum 25-hydroxyvitamin D levels are inversely associated with systemic inflammation in severe obese subjects. Internal and emergency medicine. 2013;8:33-40.

[20] Vaughan-Shaw PG, Zgaga L, Ooi LY, Theodoratou E, Timofeeva M, Svinti V, et al. Low plasma vitamin D is associated with adverse colorectal cancer survival after surgical resection, independent of systemic inflammatory response. Gut. 2020;69:103-11.

[21] van Harten-Gerritsen AS, Balvers MG, Witkamp RF, Kampman E, van Duijnhoven FJ. Vitamin D, inflammation and colorectal cancer progression: A review of mechanistic studies and future directions for epidemiological studies. Cancer Epidemiology and Prevention Biomarkers. 2015:cebp. 0601.2015.

[22] Klampfer L. Cytokines, inflammation and colon cancer. Current cancer drug targets. 2011;11:451-64.

[23] Winkels RM, Heine-Broring RC, van Zutphen M, van Harten-Gerritsen S, Kok DE, van Duijnhoven FJ, et al. The COLON study: Colorectal cancer: Longitudinal, Observational study on Nutritional and lifestyle factors that may influence colorectal tumour recurrence, survival and quality of life. BMC Cancer. 2014;14:374. 
[24] van Roekel EH, Bours MJ, de Brouwer CP, Ten Napel H, Sanduleanu S, Beets GL, et al. The Applicability of the International Classification of Functioning, Disability, and Health to Study Lifestyle and Quality of Life of Colorectal Cancer Survivors. Cancer epidemiology, biomarkers \& prevention : a publication of the American Association for Cancer Research, cosponsored by the American Society of Preventive Oncology. 2014;23:1394405.

[25] van den Ouweland JM, Beijers AM, van Daal H. Overestimation of 25-hydroxyvitamin D3 by increased ionisation efficiency of 3-epi-25-hydroxyvitamin D3 in LC-MS/MS methods not separating both metabolites as determined by an LC-MS/MS method for separate quantification of 25-hydroxyvitamin D3, 3-epi-25hydroxyvitamin D3 and 25-hydroxyvitamin D2 in human serum. Journal of Chromatography B. 2014;967:195-202. [26] de Jager W, Bourcier K, Rijkers GT, Prakken BJ, Seyfert-Margolis V. Prerequisites for cytokine measurements in clinical trials with multiplex immunoassays. BMC immunology. 2009;10:52.

[27] Meyer K, Ueland PM. Targeted quantification of C-reactive protein and cystatin c and its variants by immuno-MALDI-MS. Analytical chemistry. 2014;86:5807-14.

[28] Wendel-Vos GC, Schuit AJ, Saris WH, Kromhout D. Reproducibility and relative validity of the short questionnaire to assess health-enhancing physical activity. Journal of clinical epidemiology. 2003;56:1163-9. [29] Van Leersum N, Snijders H, Henneman D, Kolfschoten N, Gooiker G, Ten Berge M, et al. The Dutch surgical colorectal audit. European Journal of Surgical Oncology (EJSO). 2013;39:1063-70.

[30] Holick MF. Vitamin D deficiency. New England Journal of Medicine. 2007;357:266-81.

[31] Bertrand KA, Giovannucci E, Liu Y, Malspeis S, Eliassen AH, Wu K, et al. Determinants of plasma 25hydroxyvitamin $D$ and development of prediction models in three US cohorts. The British journal of nutrition. 2012;108:1889-96.

[32] Verbeke G. Linear mixed models for longitudinal data. Linear mixed models in practice: Springer; 1997. p. 63-153.

[33] Curran PJ, Bauer DJ. The disaggregation of within-person and between-person effects in longitudinal models of change. Annual review of psychology. 2011;62:583-619.

[34] Knupfer H, Preiss R. Serum interleukin-6 levels in colorectal cancer patients--a summary of published results. International journal of colorectal disease. 2010;25:135-40.

[35] Krzystek-Korpacka M, Diakowska D, Kapturkiewicz B, Bebenek M, Gamian A. Profiles of circulating inflammatory cytokines in colorectal cancer (CRC), high cancer risk conditions, and health are distinct. Possible implications for CRC screening and surveillance. Cancer letters. 2013;337:107-14.

[36] Li X, Wang Y, Han C, Li P, Zhang H. Colorectal cancer progression is associated with accumulation of Th17 lymphocytes in tumor tissues and increased serum levels of interleukin-6. The Tohoku journal of experimental medicine. 2014;233:175-82.

[37] Galizia G, Orditura M, Romano C, Lieto E, Castellano P, Pelosio L, et al. Prognostic significance of circulating IL-10 and IL-6 serum levels in colon cancer patients undergoing surgery. Clinical immunology. 2002;102:169-78. [38] Oliveira Miranda D, Soares de Lima TA, Ribeiro Azevedo L, Feres O, Ribeiro da Rocha JJ, Pereira-da-Silva G. Proinflammatory cytokines correlate with depression and anxiety in colorectal cancer patients. BioMed research international. 2014;2014:739650.

[39] Ning Y, Manegold PC, Hong YK, Zhang W, Pohl A, Lurje G, et al. Interleukin-8 is associated with proliferation, migration, angiogenesis and chemosensitivity in vitro and in vivo in colon cancer cell line models. International journal of cancer Journal international du cancer. 2011;128:2038-49.

[40] Fortis C, Foppoli M, Gianotti L, Galli L, Citterio G, Consogno G, et al. Increased interleukin-10 serum levels in patients with solid tumours. Cancer letters. 1996;104:1-5.

[41] Rosenberg-Hasson Y, Hansmann L, Liedtke M, Herschmann I, Maecker HT. Effects of serum and plasma matrices on multiplex immunoassays. Immunologic research. 2014;58:224-33.

[42] Gabay C. Interleukin-6 and chronic inflammation. Arthritis research \& therapy. 2006;8:S3.

[43] Brozek W, Bises G, Girsch T, Cross HS, Kaiser HE, Peterlik M. Differentiation-dependent expression and mitogenic action of interleukin- 6 in human colon carcinoma cells: relevance for tumour progression. European journal of cancer. 2005;41:2347-54. 


\section{CHAPTER 5}

[44] Lippitz BE. Cytokine patterns in patients with cancer: a systematic review. The lancet oncology.

2013;14:e218-28.

[45] Hsu CP, Chen YL, Huang CC, Chou CC, Liu CL, Hung CH, et al. Anti-interleukin-6 receptor antibody inhibits the progression in human colon carcinoma cells. European journal of clinical investigation. 2011;41:277-84.

[46] Chung YC, Chang YF. Serum interleukin- 6 levels reflect the disease status of colorectal cancer. Journal of surgical oncology. 2003;83:222-6.

[47] De Simone V, Franze E, Ronchetti G, Colantoni A, Fantini M, Di Fusco D, et al. Th17-type cytokines, IL-6 and TNF- $\alpha$ synergistically activate STAT3 and NF-kB to promote colorectal cancer cell growth. Oncogene.

2015;34:3493.

[48] Calton EK, Keane KN, Newsholme P, Soares MJ. The impact of vitamin D levels on inflammatory status: a systematic review of immune cell studies. PloS one. 2015;10:e0141770. 
CIRCULATING LEVELS OF 25(OH)D,3 AND INFLAMMATION MARKERS

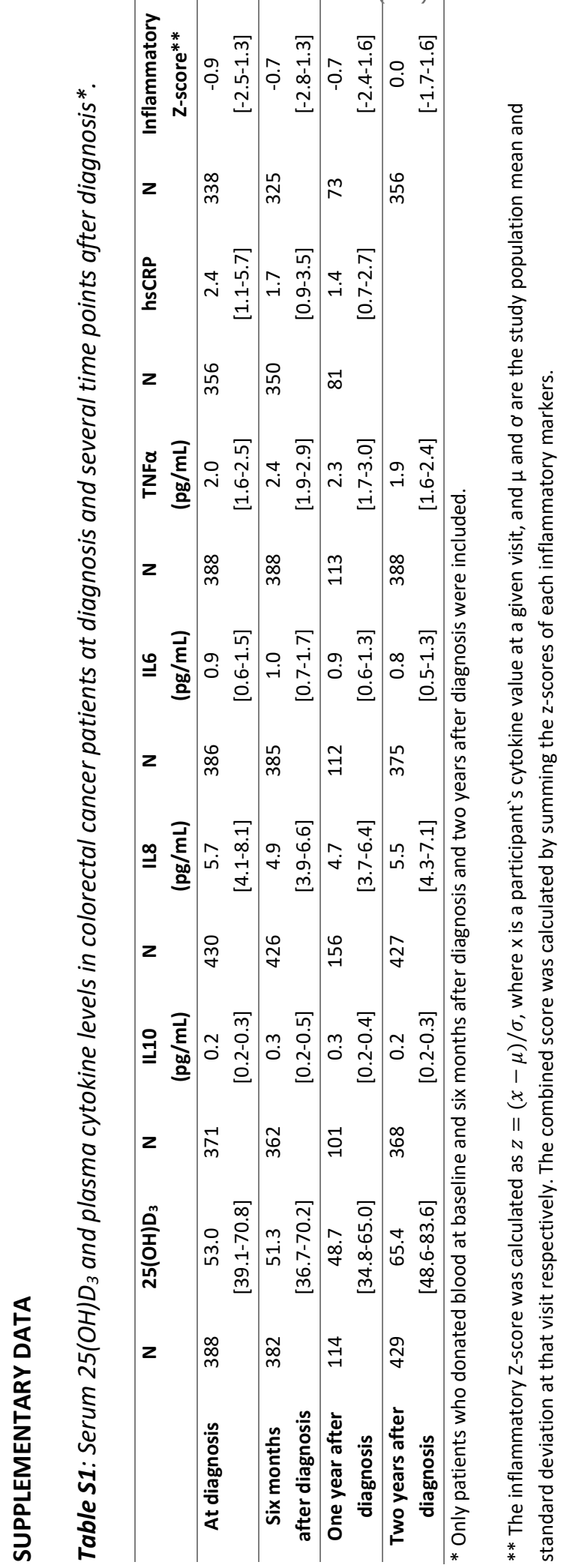





\title{
CHAPTER 6
}

\section{LEVELS OF INFLAMMATION MARKERS ARE ASSOCIATED WITH THE RISK OF RECURRENCE AND ALL-CAUSE MORTALITY IN COLORECTAL CANCER PATIENTS}

\author{
Evertine Wesselink \\ Michiel G.J. Balvers \\ Dieuwertje E. Kok \\ Renate M. Winkels \\ Moniek van Zutphen \\ Ruud W.M. Schrauwen \\ Eric T.P. Keulen \\ Ewoud A. Kouwenhoven \\ Stephanie O. Breukink \\ Renger F. Witkamp \\ Johannes H.W. de Wilt \\ Martijn J.L. Bours \\ Matty P. Weijenberg \\ Ellen Kampman
}

Franzel J.B. van Duijnhoven

Cancer Epidemiol Biomarkers \& Prevention. 2021 Mar 26;cebp.EPI-20-1752-E.2020. 


\section{ABSTRACT}

Background: We investigated whether pre-operative and post-operative levels of inflammation markers, which have mechanistically been linked to colorectal cancer (CRC) progression, were associated with recurrence and all-cause mortality in CRC patients.

Methods: Data of two prospective cohort studies were used. For the current analysis, patients with stage I-III CRC were considered. Data on inflammation (Interleukin (IL)6, IL8, IL10, Tumour Necrosis Factor (TNF) $\alpha$, high sensitivity C-reactive protein (hsCRP) and a combined inflammatory z-score) was available for 747 patients before surgery and for 614 patients after surgery. The associations between inflammation marker levels and CRC recurrence and all-cause mortality were examined using multivariable Cox proportional hazard regression models, taking into account patient characteristics, clinical- and lifestyle factors.

Results: Higher pre-operative as well as post-operative hsCRP levels were associated with a higher risk of recurrence $\left(\mathrm{HR}_{\text {per doubling }}(95 \% \mathrm{Cl}), 1.15(1.02-1.30)\right.$ and $\left.1.34(1.16-1.55)\right)$ and all-cause mortality $\left(\mathrm{HR}_{\text {per doubling }}(95 \% \mathrm{Cl}) 1.13\right.$ (1.01-1.28) and 1.15 (0.98-1.35)). A doubling in IL8 levels (pre-operative levels HR $1.2395 \% \mathrm{Cl} 1.00-1.53$ and post-operative levels HR $1.6195 \% \mathrm{Cl} 1.23-2.12$ ) and a higher combined inflammatory z-score (pre-operative $\mathrm{HR}_{\text {per }}$

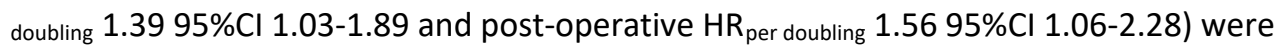
associated with a higher risk of all-cause mortality, but not recurrence. No associations between IL6, IL10 and TNF $\alpha$ and recurrence or all-cause mortality were observed.

Conclusion: Pre-operative and post-operative levels of specific inflammation markers were associated with recurrence and/or all-cause mortality.

Impact: The complex role of inflammation in cancer recurrence merits further elucidation by investigating local inflammation at the tumour-site.

Keywords: colorectal cancer, recurrence, all-cause mortality, cytokines, inflammation 


\section{INTRODUCTION}

Chronic low-grade inflammation is an important driver of cancer development and progression [1-4]. This includes colorectal cancer (CRC) [2, 5]. Vice versa, non-steroidal anti-inflammatory drugs (NSAIDs) and cytokine antagonist or antibodies may play a role in the prevention and treatment of $\operatorname{CRC}[2,5,6]$. Together, this underlines the potential relevance of inflammation during tumour onset and progression.

The association between C-reactive protein (CRP), a nonspecific marker of inflammation, and CRC prognosis has been extensively studied [7-9]. Higher CRP levels were associated with a higher risk of recurrences [9] and a worse disease-free-, cancer specific- and overall survival $[7,8]$. Next to CRP, more specific inflammation markers have also been studied, e.g. cytokines including several interleukins (IL) and Tumour Necrosis Factor alpha (TNF $\alpha$ ), suggested crucial players in CRC tumour immunology $[2,5,10]$. Higher levels of IL6, TNF $\alpha$, CRP, and IL8 have been associated with more advanced CRC [11-15]. Likewise, higher levels of IL6, IL8, IL10 and TNF $\alpha$ have also been associated with a worse disease free-, cancer specific- and overall survival [11, 14, 16-20].

So far, studies investigating potential associations between cytokines and CRC involved relatively small numbers of subjects, usually between 50 and 250 . Importantly, those studies only measured cytokine levels at diagnosis and thus before surgery. The timing of measurements could be important, since cytokine levels might be different in the preoperative phase when the tumour is still present and there is active interaction with the tumour microenvironment and host immunity, this is not the case when the tumour is removed [5]. In addition, previous studies did not adjust for lifestyle and other clinical factors not directly linked to the tumour itself. Several lifestyle factors or other patient characteristics, such as physical activity, BMI, smoking and dietary habits, comorbidities, NSAID use and vitamin D status are of potential interest as they are associated with both inflammation as well as mortality [21-30].

In view of these knowledge gaps and considerations, we investigated pre-operative as well as post-operative levels of IL6, IL8, IL10, TNF $\alpha$ and CRP levels in association with cancer recurrence and all-cause mortality, while taking lifestyle and clinical factors into account, in a large study of 747 CRC patients. 


\section{METHODS}

\section{STUDY DESIGN AND POPULATION}

Data from two prospective cohort studies were used, the COLON study and the EnCoRe study. The design of the COLON study [31] (ClinicalTrials.gov identifier:NCT03191110) and the EnCoRe study [32] (trialregister.nl identifier:NL6904) have been described elsewhere. Briefly, in both studies, newly diagnosed CRC patients were recruited directly after diagnosis and were followed during and after treatment. Men and women above the age of 18 were eligible. In the COLON study, patients with a first primary stage I-IV CRC were eligible, while patients with primary stage I-III CRC were eligible in the EnCoRe study. The COLON and EnCoRe studies were approved by regional Medical Ethics Committees (NL30446.091.09 and NL38786.068.11, respectively). All patients signed written informed consent.

In total 1,089 patients were recruited of which 791 in the COLON study and 298 in the EnCoRe study (Figure 1). For 232 patients no pre-operative blood samples were available, mainly because of the small time-window between diagnosis, study inclusion and surgery. Patients with missing data about stage of disease $(n=57)$ or who had stage IV disease $(n=52)$ were also excluded. In total 747 patients were included in the analyses of the association between pre-operative levels and all-cause mortality. For 133 patients data on inflammation markers was missing six months after diagnosis (post-operative). For the association between post-operative levels and all-cause mortality 614 patients were included. Data about recurrence was missing for 7 patients for the pre-operative analyses and for 5 patients for the post-operative analyses.

\section{BLOOD COLLECTION}

In principle, blood samples were obtained during a regular clinical visit to the hospital at diagnosis and six months after diagnosis (median 5.8 IQR 5.4-6.4 months) in the COLON study and during a regular clinical visit to the hospital or by a research assistant during a home visit before start of cancer treatment and 6 weeks and 6 months after cancer treatment in the EnCoRe study. To be able to harmonize the data of both cohorts for analyses after CRC diagnosis, we selected the time point in the EnCoRe cohort closest to six months after diagnosis (median 6.9 IQR 6.4-8.5 months).

All blood samples were centrifuged and aliquoted and plasma was stored at $-80^{\circ} \mathrm{C}$ until further analysis. 


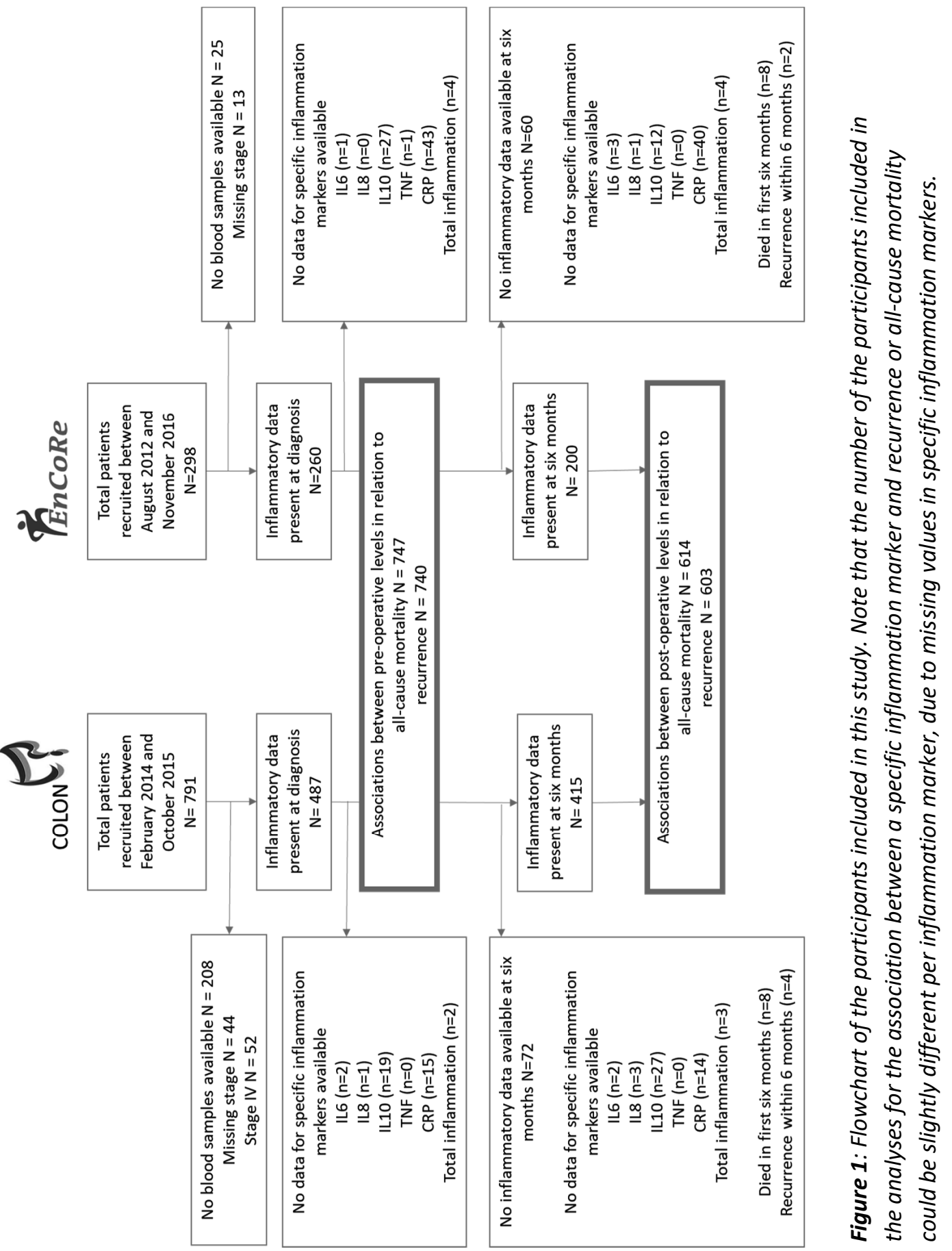




\section{INFLAMMATION MARKERS}

The pro-inflammatory panel 1 (human) kit from MSD cytokine multiplex assays (Meso Scale Diagnostics, Rockville, MD, USA) was used to measure IL6, IL8, IL10 and TNFa in plasma in our lab at Wageningen University \& Research. These inflammatory markers has been selected based on their suggested role in CRC progression [29]. Measurements of cytokines were done in duplicate according to the manufacturer's protocol. Highest inter and intra-batch coefficients of variation for the manufacturer's quality controls for all cytokines were $<8 \%$, and reported values deviated no more than $15 \%$ from the assigned target values. Detailed information about the analytical methods has been published previously [30]. High sensitive C-reactive protein (hsCRP) was measured using an immunoMALDI mass spectrometry method [33] (BEVITAL, Bergen, Norway). The inter-assay coefficient ranged between 3 and $6 \%$.

A combined inflammatory z-score (including hsCRP, TNF, IL6, IL8 and IL10) was calculated [34] to cluster conceptually related markers of low-grade inflammation and improve statistical efficiency. First, a normalized $z$-score for each individual biomarker was calculated. The combined score was calculated by summing the z-scores of each individual marker and dividing the total sum by the number of inflammation markers included. If data of two or more inflammatory markers were missing for a patient $(n=6$ pre-operative and $n=7$ post-operative), no combined inflammatory $z$-score was calculated.

\section{COLORECTAL CANCER OUTCOMES}

Information on recurrence was collected from medical records by the Dutch Cancer Registration. Recurrence was defined as a local regional recurrence or distant metastasis. Follow-up time for recurrence was calculated starting from the date of blood collection, either preoperative or post-operative, until the date of recurrence or until the date recurrence status was updated (COLON: February 2018; EnCoRe: March 2018), or the date of end of follow-up, whichever came first.

Information on all-cause mortality was gathered from linkage with the Municipal Personal Record Database. Follow-up time was defined starting from the date of blood collection, either preoperative or post-operative, until the date of death, or until the last date survival status was updated (COLON: December 2019; EnCoRe: May 2019), whichever came first.

\section{COVARIATE ASSESSMENT}

Information on demographics, smoking, physical activity, diet, use of dietary supplements and NSAIDs was obtained using self-administered questionnaires. Physical activity was assessed using the Short Questionnaire to Assess Health-enhancing physical activity 
(SQUASH) [35]. Information on height, weight, and waist and hip circumference was collected using self-administered questionnaires in the COLON study. In the EnCoRe study, these measurements were performed by trained research dieticians during home visits. Habitual dietary intake in the month (COLON) or year (EnCoRe) preceding diagnosis was assessed using a semi-quantitative food frequency questionnaire (FFQ). The FFQ used in the COLON study consists of 204 items. The FFQ used in the EnCoRe study consists of 253 items. Six months after diagnosis habitual dietary intake was assessed with a FFQ in the COLON study and a 7-day food diary in the EnCoRe study.

Clinical factors, such as stage of disease, tumour location, tumour differentiation, date of start treatment, type of treatment and presence of comorbidities were derived from the Dutch ColoRectal Audit (DCRA) [36].

For both cohorts, serum 25-hydroxy vitamin $\mathrm{D}_{3}\left(25(\mathrm{OH}) \mathrm{D}_{3}\right)$ levels were measured by liquid chromatography tandem mass spectrometry (LC-MS/MS) in the Canisius Wilhelmina Hospital, Nijmegen, the Netherlands.

\section{DATA ANALYSES}

This analysis included 747 stage I-III CRC patients of whom data of levels of inflammation markers was available at diagnosis. For 614 patients data about levels of inflammation six months after diagnosis was available.

Population characteristics are described as numbers with percentages and medians with interquartile range (IQR).

Interleukin-6, IL8, IL10, TNF $\alpha$, hsCRP and the combined inflammatory z-score were the exposure variables in this study. Inflammation markers were log2 transformed to obtain normally distributed data. Hazard ratios from continuous analyses should therefore be interpreted as the increased risk with a doubling in the levels of an inflammation marker.

To visualize the relationship between inflammation and CRC outcomes, Restricted Cublic Splines (RCS) was used (SAS Macro \%RCS_Reg vs1.44). Three knots were placed on the 10th, 50th and 90th percentile, where the 50th percentile was used as the reference. The associations between the majority of inflammation markers and outcomes were linear (Figure S1). Therefore, the association between continuous inflammation markers and CRC outcomes was investigated using Cox proportional hazard models.

First, a crude Cox proportional hazard model, including only the main exposure of interest (i.e. IL6, IL8, IL10, TNF $\alpha$, hsCRP or the combined inflammatory z-score) and the outcome (i.e. recurrence or all-cause mortality) was run (model 1). Second, based on literature, analyses were adjusted for age, sex, BMI, stage of disease (I/II/III), season of blood 
collection and cohort (model 2) [7, 9, 37]. Additionally, other clinical and lifestyle factors were investigated for confounding; tumour location (colon/rectal), differentiation grade (good/moderate, bad), daily use of NSAIDs (yes/no), daily use of statins (yes/no), having comorbidities (yes/no), received treatment (only surgery / surgery and chemotherapy / surgery and radiotherapy / surgery and chemoradiation), neo-adjuvant treatment (yes/no), adjuvant treatment (yes/no), number of days between surgery and postoperative blood sampling, hours of moderate to vigorous physical activity per week (continuous), smoking habits (current/former/never), education level (low/medium/high), intake of red and processed meat (g/day), intake of vegetables and fruits (g/day), fibre intake (g/day) and alcohol intake (g/day). Only smoking habits changed the association of model 2 with more than $10 \%$ and this was added to the fully adjusted model (model $2+$ smoking).

Above mentioned associations were examined with data from two time points: preoperative (at diagnosis, in $92 \%$ of the participants before the start of treatment) and postoperative (six months after diagnosis, in $83 \%$ of the participants after the end of treatment). Confounding by modifiable lifestyle factors such as dietary intake, physical activity levels and BMI was evaluated using data assessed at the same time-point as blood measurements were performed. Correlations between pre-operative and post-operative levels of inflammatory markers were examined using linear regression analyses.

Sensitivity analyses were done: 1) for NSAID non-users only; 2) for non-smokers only; 3 ) excluding patients who received (neo)adjuvant treatment (for pre-operative levels patients who received neoadjuvant treatment were excluded; for post-operative levels patients who received adjuvant treatment were excluded; 4) excluding patients still undergoing treatment (for pre-operative levels patients who already started treatment were excluded; for post-operative levels patients who still underwent adjuvant treatment were excluded; 5) excluding outliers of inflammatory markers (>3SD above the mean); 6) excluding older blood samples (levels measured after two years of storage, since cytokines were previously shown to remain stable in plasma for a period up to 2 years of storage at $80^{\circ} \mathrm{C}$ [38]); 7) correction for pre-operative or post-operative levels and 8) excluding patients with hsCRP levels $>10 \mathrm{mg} / \mathrm{mL}$.

To further investigate the interference of conditions influencing inflammation, such as other comorbidities $[27,28]$ and vitamin D status $[29,30]$, we tested for multiplicative interaction between inflammation and having any comorbidities, specifically cardiometabolic comorbidities and vitamin D status in relation to CRC outcomes. Multiplicative interaction was assessed using the interaction term approach. Thus, the exposure (inflammation marker levels) and the above mentioned potential effect modifiers were entered as a product term in the model e.g. IL6*vitamin status. We did not stratify our analyses on vitamin D status and comorbidities, since all $p$ for interaction 
values were above 0.05 . The only exception was the interaction between pre-operative TNF $\alpha$ levels, vitamin D status and CRC recurrence $p=0.005$. However, although statistically significant, this finding might be a chance finding, therefore we did not stratify analyses for vitamin D status.

All statistical analyses were performed in SAS, version 9.4. P-values $<0.05$ were considered statistically significant.

\section{RESULTS}

\section{POPULATION CHARACTERISTICS}

Mean age of the total population was 67 (IQR 62-73) years, 33\% was female and $44 \%$ of the patients were diagnosed with stage III of disease (Table 1). Compared to patients with a low combined inflammatory z-score (tertile 1), patients with a high combined inflammatory z-score (tertile 3) were older, had a higher BMI, more often had a low education level, were less physically active, more often had colon cancer, were more often in stage II of disease, more often had comorbidities and had lower levels of $25(\mathrm{OH}) \mathrm{D}_{3}$ (Table 1).

Post-operative samples were obtained 5 to 6 months after surgery in the majority of patients (median 174 days, IQR 151-194). A moderately strong correlation between pre and post-operative inflammatory marker levels was observed (IL6, r=0.4; IL8, r=0.6; IL10, $r=0.4$; TNF $\alpha, r=0.6$; hsCRP, $r=0.4$, combined inflammatory $z$-score, $r=0.4$ ). Levels of inflammation markers did not change substantially (>1SD) between diagnosis and six months after diagnosis in $77 \%$ of our study population. In $13 \%$ of the study population levels increased with more than 1SD, while in 10\% levels decreased with more than 1SD. Characteristics of patients with increased, stable and decreased levels are depicted in table S1.

During a median follow-up of 3.0 years [IQR 2.4-3.5] for recurrence and 4.7 years [IQR 4.25.3] for all-cause mortality, 88 recurrences and 98 deaths occurred. For the post-operative measurements, the median follow-up was 2.5 years [IQR 2.0-3.0] for recurrence and 4.3 years [IQR 3.8-4.9] for all-cause mortality, in which 64 recurrences and 61 deaths occurred. 
Table 1 Baseline characteristics of colorectal cancer patients stratified by tertiles of the combined inflammatory z-score*

\begin{tabular}{|c|c|c|c|c|}
\hline & Total population & $\begin{array}{l}\text { Low level of } \\
\text { inflammation } \\
\text { (tertile 1) } \\
\mathrm{N}=\mathbf{2 4 7}\end{array}$ & $\begin{array}{l}\text { Moderate level of } \\
\text { inflammation } \\
\text { (tertile 2) } \\
\mathrm{N}=\mathbf{2 4 8}\end{array}$ & $\begin{array}{c}\text { High level of } \\
\text { inflammation } \\
\text { (tertile 3) } \\
\mathrm{N}=\mathbf{2 4 7}\end{array}$ \\
\hline Age (years) & $67.2(62.1-73.4)$ & $65.6(60.3-70.2)$ & $67.0(61.6-73.0)$ & $68.9(64.2-75.2)$ \\
\hline Gender (female) & $245(33)$ & $79(32)$ & $80(32)$ & $84(34)$ \\
\hline BMI $\left(\mathrm{kg} / \mathrm{m}^{2}\right)$ & $26.8(24.3-29.7)$ & $25.9(23.9-28.9)$ & $27.2(24.8-30.1)$ & $27.6(24.5-30.9)$ \\
\hline Unknown & 3 & 0 & 1 & 2 \\
\hline \multicolumn{5}{|l|}{ Education $^{a}$} \\
\hline Low & 347 (48) & $107(44)$ & $110(46)$ & $128(55)$ \\
\hline Medium & $179(25)$ & $66(27)$ & $58(24)$ & $54(23)$ \\
\hline High & $193(27)$ & $68(28)$ & $70(29)$ & $52(22)$ \\
\hline Unknown & 29 & 6 & 10 & 13 \\
\hline \multicolumn{5}{|l|}{ Smoking habits } \\
\hline Current & $95(13)$ & $31(13)$ & $29(12)$ & $35(15)$ \\
\hline Former & $429(58)$ & $146(60)$ & $143(59)$ & $137(57)$ \\
\hline Never & 212 (29) & $68(28)$ & $71(29)$ & $70(29)$ \\
\hline Unknown & 12 & 2 & 5 & 5 \\
\hline \multicolumn{5}{|l|}{ Physical activity ${ }^{\text {b }}$} \\
\hline (hours/week) & $11.3(5.0-20.0)$ & $12.6(6.0-21.4)$ & $10.5(4.5-19.5)$ & $10.3(4.0-19.0)$ \\
\hline Unknown & 13 & 3 & 5 & 5 \\
\hline \multicolumn{5}{|l|}{ Dietary factors $^{c}$} \\
\hline Fruits & $135(57-225)$ & $151(57-226)$ & $114(46-224)$ & $142(71-223)$ \\
\hline Vegetables & $112(71-171)$ & $114(75-174)$ & $106(69-165)$ & $114(73-170)$ \\
\hline Fibre & $22(17-27)$ & $22(18-27)$ & $21(17-27)$ & $22(17-27)$ \\
\hline Red meat & $41(25-61)$ & $41(26-61)$ & $42(25-62)$ & $41(24-61)$ \\
\hline Processed meat & $30(13-47)$ & $30(13-48)$ & $30(15-46)$ & $30(12-46)$ \\
\hline Alcohol & $9(1-21)$ & $12(2-23)$ & $9(1-21)$ & $5(0-19)$ \\
\hline Unknown & 17 & 4 & 7 & 6 \\
\hline \multicolumn{5}{|l|}{ Type of cancer } \\
\hline Colon & $491(66)$ & $156(63)$ & $159(64)$ & $174(70)$ \\
\hline Rectum & $257(34)$ & $91(37)$ & $89(36)$ & $73(30)$ \\
\hline \multicolumn{5}{|l|}{ Tumour stage } \\
\hline I & $216(29)$ & $92(37)$ & $65(26)$ & $57(23)$ \\
\hline II & $206(28)$ & $51(21)$ & $68(27)$ & $86(35)$ \\
\hline III & $326(44)$ & $104(42)$ & $115(46)$ & $104(42)$ \\
\hline \multicolumn{5}{|l|}{ Type of } \\
\hline Only surgery & $158(21)$ & $48(20)$ & $53(21)$ & $57(23)$ \\
\hline $\begin{array}{c}\text { Surgery and } \\
\text { chemotherapy }\end{array}$ & $87(12)$ & $31(13)$ & $29(12)$ & $25(10)$ \\
\hline $\begin{array}{l}\text { Surgery and } \\
\text { radiotherapy } \\
\text { Surgery and }\end{array}$ & $71(9)$ & $20(8)$ & $21(9)$ & $29(12)$ \\
\hline $\begin{array}{l}\text { chemo radiation } \\
\text { Unknown }\end{array}$ & 16 & 7 & 5 & 4 \\
\hline
\end{tabular}




\begin{tabular}{|c|c|c|c|c|}
\hline $\begin{array}{c}\text { Use of NSAIDs } \\
\text { (yes) }\end{array}$ & $135(18)$ & $39(16)$ & $56(23)$ & 40 (17) \\
\hline \multicolumn{5}{|l|}{ Comorbidities } \\
\hline Yes & $561(75)$ & $168(68)$ & $192(78)$ & $197(80)$ \\
\hline Unknown & 2 & 0 & 1 & 1 \\
\hline $\begin{array}{l}\text { Serum } 25(\mathrm{OH}) D_{3} \\
\text { levels (nmol/L) }\end{array}$ & $53.7(40.3-68.9)$ & $57.1(42.4-74.0)$ & $52.5(39.1-67.1)$ & $51.8(38.2-68.0)$ \\
\hline \multicolumn{5}{|l|}{ Season of blood } \\
\hline \multicolumn{5}{|l|}{ collection $^{d}$} \\
\hline Spring & $213(28)$ & $78(32)$ & $62(25)$ & $73(30)$ \\
\hline Summer & $220(29)$ & $72(29)$ & $66(27)$ & $80(32)$ \\
\hline Autumn & $138(18)$ & $42(17)$ & $48(19)$ & 47 (19) \\
\hline Winter & $177(24)$ & $55(22)$ & $72(29)$ & $47(19)$ \\
\hline \multicolumn{5}{|l|}{$\begin{array}{l}\text { Inflammatory } \\
\text { markers }\end{array}$} \\
\hline IL6 (pg/ml) & $1.0(0.7-1.6)$ & $0.7(0.5-0.9)$ & $1.0(0.8-1.4)$ & $1.8(1.2-3.1)$ \\
\hline IL8 (pg/ml) & $5.7(4.3-8.1)$ & $4.3(3.3-5.4)$ & $5.6(4.2-7.4)$ & $8.7(6.5-12.9)$ \\
\hline IL10 (pg/ml) & $0.2(0.2-0.3)$ & $0.2(0.1-0.2)$ & $0.2(0.2-0.3)$ & $0.3(0.3-0.6)$ \\
\hline TNF (pg/ml) & $2.1(1.6-2.6)$ & $1.6(1.3-1.9)$ & $2.0(1.7-2.5)$ & $2.6(2.2-3.3)$ \\
\hline CRP $(\mu \mathrm{g} / \mathrm{ml})$ & $2.5(1.2-6.1)$ & $1.1(0.6-2.0)$ & $2.5(1.4-4.1)$ & $7.8(3.6-16.9)$ \\
\hline Combined & -0.08 & -0.60 & -0.08 & 0.62 \\
\hline $\begin{array}{l}\text { inflammatory z- } \\
\text { score }\end{array}$ & $(-0.44-0.38)$ & $(-0.81--0.44)$ & $(-0.21-0.05)$ & $(0.40-0.98)$ \\
\hline \multicolumn{5}{|l|}{ Cohort } \\
\hline COLON & $487(65)$ & $172(70)$ & 169 (68) & $144(58)$ \\
\hline EnCoRe & $261(35)$ & $75(30)$ & $79(32)$ & $103(42)$ \\
\hline Deceased & $98(13)$ & $20(8)$ & $35(14)$ & $42(17)$ \\
\hline Recurrence & $88(11)$ & $24(10)$ & $34(14)$ & $30(12)$ \\
\hline
\end{tabular}

Values presented are median (quartile 1 - quartile 3) or number (percentages). Abbreviations: 25(OH) D3, 25hydroxyvitamin D3; NSAIDs, non-steroid anti-inflammatory drugs. *Low, moderate and high levels of inflammation were derived from the tertiles of the combined inflammatory-z-scores of IL 6 + IL8 + IL10 + TNF $\alpha+$ CRP /5. For 81 patients, levels of one inflammation marker was missing. For these patients the main inflammatory $z$-score was calculated by; summing the $z$-scores of the four available inflammation markers $/ 4$. For 5 patients more than one inflammatory marker was missing. For these patients no mean inflammatory z-score was calculated. Cut-off points for the tertiles of the total inflammation level were: -0.32 and 0.18 .

a: Low education was defined as primary school and lower general secondary education; medium as lower vocational training and higher general secondary education; high as high vocational training and university. b: Activities with a Metabolic Equivalent score (MET score) $\geq 3$ were defined as moderate to vigorous physical activity.

c: Intake in grams per day.

d: Spring: March-May; Summer: June-August; Autumn: September-November; Winter: December-February. 


\section{INFLAMMATION MARKERS, RECURRENCE AND ALL-CAUSE MORTALITY}

A doubling in pre-operative hsCRP levels was associated with a $15 \%$ higher risk of CRC recurrence (HR 1.15 95\% Cl 1.02-1.30) and a doubling in post-operative hsCRP levels was associated with a $34 \%$ higher risk of CRC recurrence (HR $1.3495 \% \mathrm{Cl} 1.16-1.55)$. Doubling of pre- as well as post-operative cytokine levels (IL6, IL8, IL10 and TNF $\alpha$ ) and the combined inflammatory z-score were not associated with cancer recurrence (Figure 2).

For all-cause mortality, pre-operative as well as post-operative levels of pro-inflammatory markers i.e. IL6, IL8, TNF $\alpha$ and hSCRP, tended to be associated with a higher risk (Figure 2). These associations were statistically significant for both pre- and post-operative levels of IL8 (HR 1.23 95\% Cl 1.00-1.53 and HR 1.61 95\% Cl 1.23-2.12, respectively) and the combined inflammatory z-score (HR $1.3995 \% \mathrm{Cl} 1.03-1.89$ and $\mathrm{HR} 1.5695 \% \mathrm{Cl}$ 1.06-2.28, respectively) and for pre-operative levels of hsCRP (HR $1.1395 \% \mathrm{Cl} 1.01-1.28)$. Levels of IL10 were not associated with all-cause mortality. 


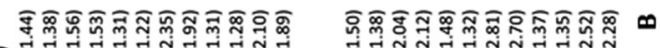

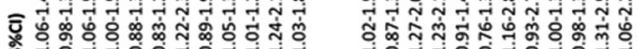

o

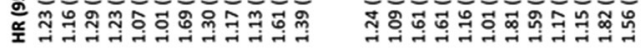

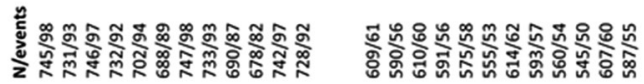
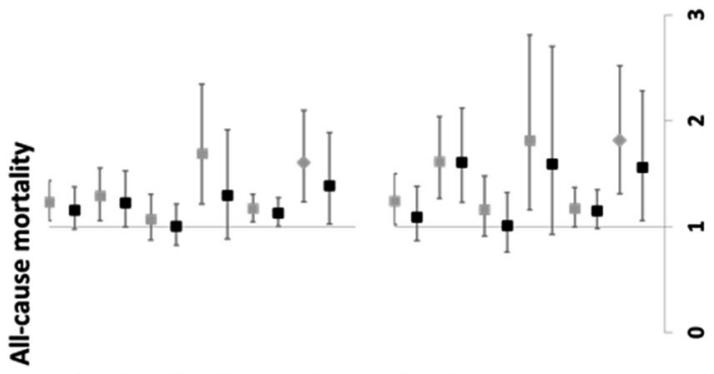

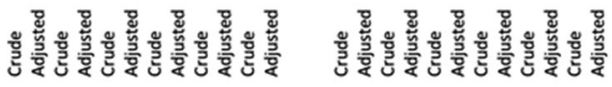

agation

密

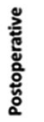

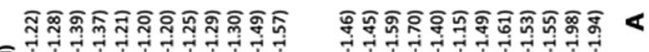

б

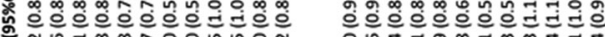

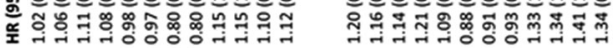

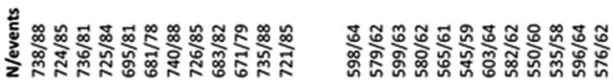

品

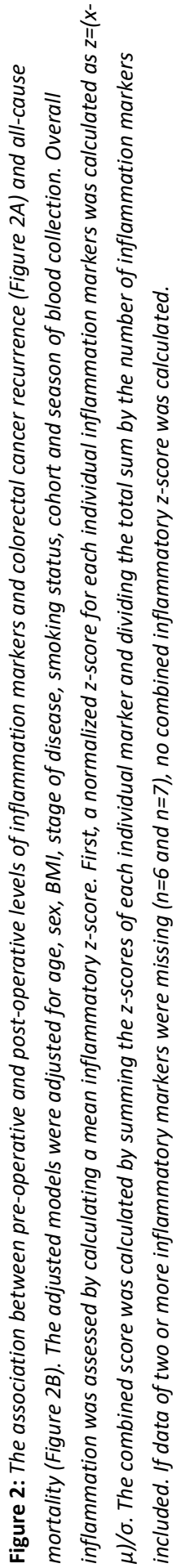

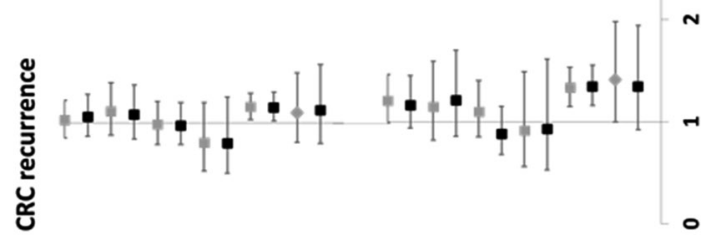

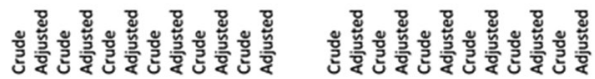

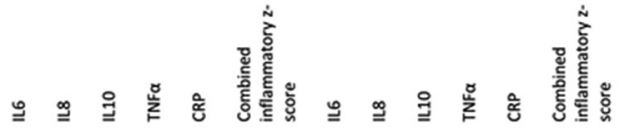




\section{RESULTS OF SENSITIVITY ANALYSES}

No differences in associations with recurrence and all-cause mortality were observed when non-NSAID users, non-smokers, patients receiving no treatment at the moment of blood collection, and patients not receiving (neo)adjuvant treatment were compared with the total population (Figure 3 and 4). Also, no differences were observed when excluding old blood samples or outliers. Associations between post-operative levels of inflammation markers and recurrence and all-cause mortality did not markedly change after adjustment for pre-operative levels. The association between pre-operative levels and CRC outcomes diminished after correction for post-operative levels. No differences were observed for the associations between cytokines and CRC outcomes and hsCRP and recurrence when excluding patients with acute infections ( $\mathrm{hsCRP}>10 \mu \mathrm{g} / \mathrm{ml}$ ). However, the association between post-operative hsCRP levels and all-cause mortality disappeared. 


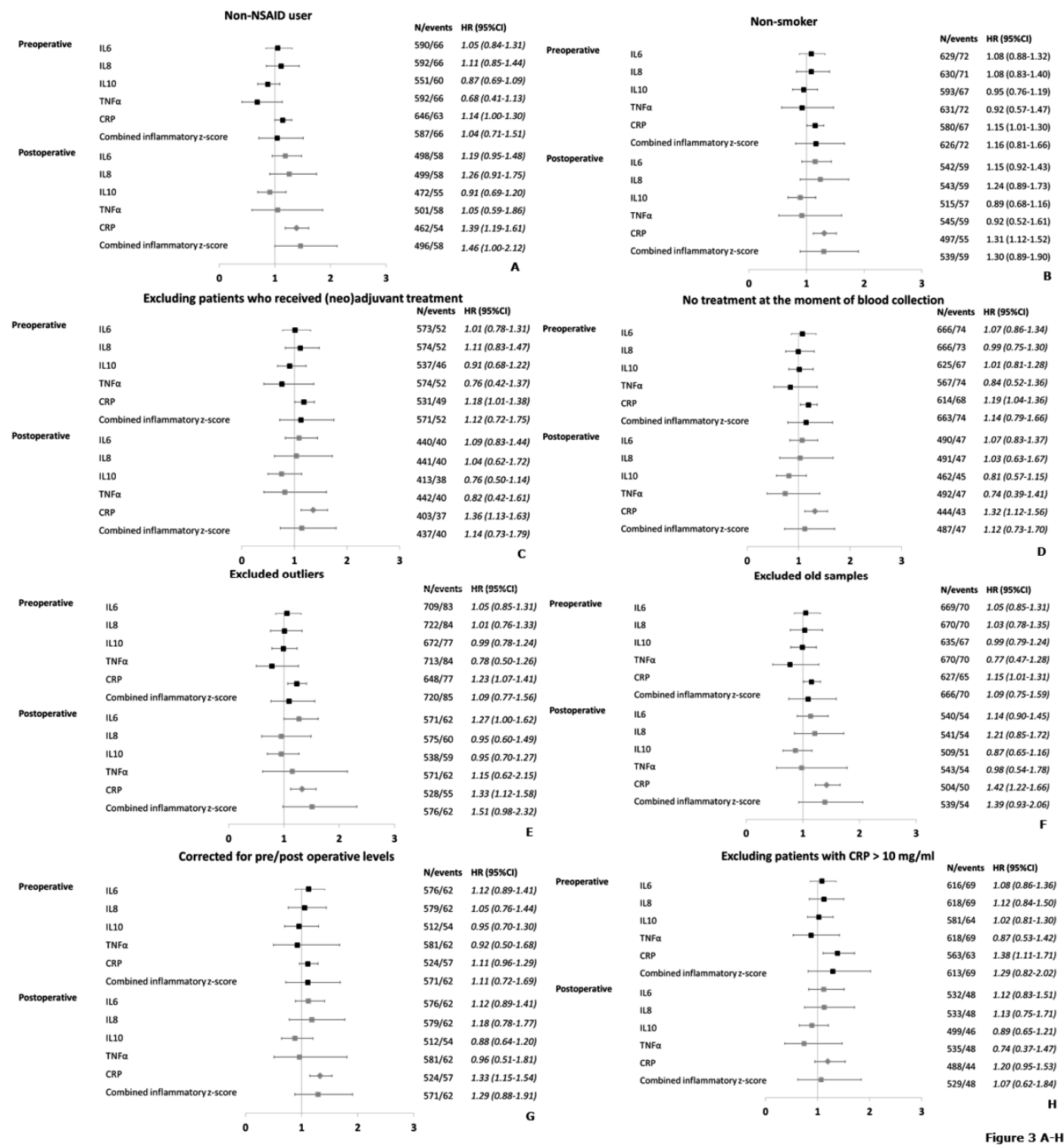

Figure 3 Results of sensitivity analyses regarding the association between inflammation markers and recurrence, adjusted for age, sex, BMI, stage of disease, smoking status, cohort and season of blood collection. Sensitivity analyses were done for: 1) only NSAID non-users (Figure 3A), 2) only non-smokers (Figure 3B), 3) excluding patients who received (neo)adjuvant treatment; for pre-operative levels patients who received neoadjuvant treatment were excluded; for post-operative levels patients who received adjuvant treatment were excluded (Figure 3C), 4) excluding patients still undergoing treatment (for pre-operative levels patients who already started treatment were excluded, for post-operative levels patients who still underwent adjuvant treatment were excluded (Figure 3D), 5) excluding outliers; biomarkers more than 3SD below or above the mean (Figure 3E), 6) excluding old samples; levels measured after two years of storage (Figure 3F). 7) correction for pre-operative or post-operative levels (Figure 3G) and 8) excluding patients with hsCRP levels $>10 \mathrm{mg} / \mathrm{mL}$ (Figure $3 \mathrm{H}$ ). 


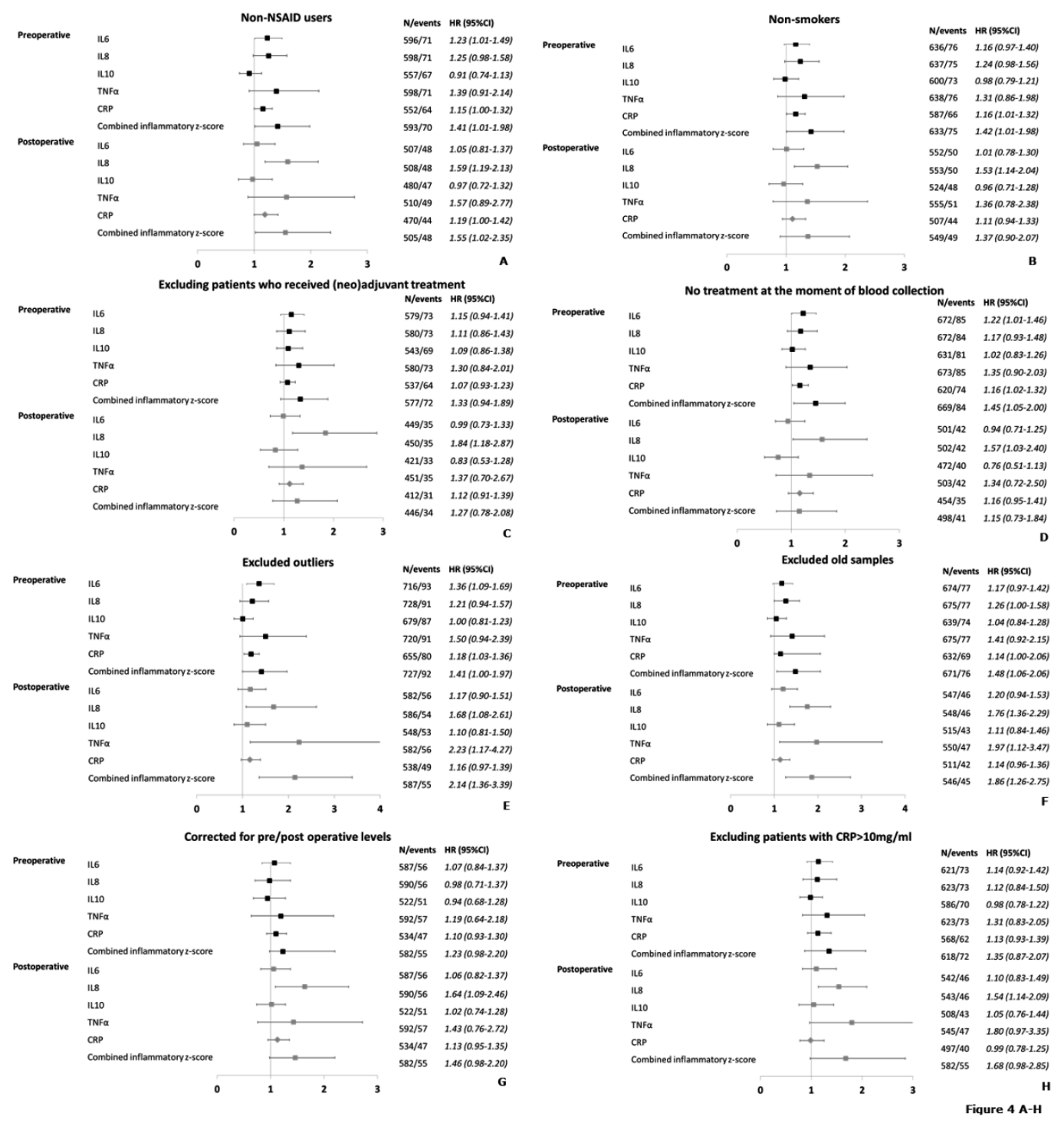

Figure 4: Results of sensitivity analyses regarding the association between inflammation markers and all-cause mortality, adjusted for age, sex, $B M I$, stage of disease, smoking status, cohort and season of blood collection. Sensitivity analyses were done for: 1) only NSAID non-users (Figure 4A), 2) only non-smokers (Figure 4B), 3) excluding patients who received (neo)adjuvant treatment; for pre-operative levels patients who received neoadjuvant treatment were excluded; for post-operative levels patients who received adjuvant treatment were excluded (Figure 4C), 4) excluding patients still undergoing treatment; for pre-operative levels patients who already started treatment were excluded, for post-operative levels patients who still underwent adjuvant treatment were excluded (Figure 4D), 5) excluding outliers; biomarkers more than 3SD below or above the mean (Figure 4E), 6) excluding old samples; levels measured after two years of storage (Figure 4F). 7) correction for preoperative or post-operative levels (Figure 4G) and 8) excluding patients with hsCRP levels $>10 \mathrm{mg} / \mathrm{mL}$ (Figure $4 H$ ). 


\section{DISCUSSION}

Associations between preoperatively and postoperatively analysed inflammation marker levels and CRC outcomes were comparable. A doubling in hsCRP levels was associated with a higher risk of recurrence. No associations between IL6, IL8, IL10, TNF $\alpha$ and the combined inflammatory z-score and recurrence were observed. Higher levels of IL8, hsCRP and the combined inflammatory z-score were associated with a higher risk of all-cause mortality. Post-operative levels of IL6 and TNF $\alpha$ tended to be associated with all-cause mortality, while no associations were observed for IL10.

We observed no striking differences between associations with pre-operative levels compared to post-operative levels and CRC outcomes. Levels of inflammation markers did not substantially change over time. Together, results indicate that low-grade systemic inflammation is associated with CRC recurrence (hsCRP) and all-cause mortality, independently of the presence of the primary tumour. Interestingly, the association between pre-operative levels of inflammation markers and CRC outcomes diminished after correction for post-operative levels, while this did not happen the other way around. These results indicate that post-operative levels of inflammation marker levels were more strongly associated with mortality compared to pre-operative levels.

To our knowledge, our study is the largest study that assessed the association between systemic levels of several cytokines mechanistically linked to CRC tumour immunology [5] and $C R C$ recurrence. We observed a higher risk of recurrence with higher pre-operative as well as post-operative hsCRP levels (median pre-operative levels $2.5 \mu \mathrm{g} / \mathrm{ml}$ IQR 1.2-6.1 and post-operative levels $1.8 \mu \mathrm{g} / \mathrm{ml}$ IQR 0.9-4.1). This is in line with results of previous studies. A previous prospective cohort study in 167 CRC patients observed a higher risk of recurrence with higher pre-operative hsCRP levels (>10 $\mu \mathrm{g} / \mathrm{ml}$ versus $<10 \mu \mathrm{g} / \mathrm{ml}$ ) although this was not statistically significant [39]. Although we did not observe an association between IL6, TNF $\alpha$ and IL10 and recurrence, 4 small previous studies observed a higher risk of recurrence with high compared to low IL6 levels [11, 40] , TNF $\alpha$ levels [11] or IL10 levels [41]. Thus, data is still somewhat inconsistent about the associations between cytokine levels and CRC recurrence and more research is needed to further elucidate the role of inflammatory cytokines in CRC recurrence. To further explore this, future research should investigate local inflammation in the tumour microenvironment, as well as circulating levels, preferably several times, before, during and after treatment [42, 43].

All pro-inflammatory markers (IL6, IL8, TNF $\alpha$, hsCRP) measured tended to be associated with a higher risk of all-cause mortality, but this was only statistically significant for IL8 and the combined inflammatory z-score. No associations were observed for the antiinflammatory cytokine IL10. The higher risk of all-cause mortality with higher IL8 levels is in line with results of previous studies [20]. In a meta-analysis including four studies with 
in total 330 patients, high pre-operative serum IL8 levels compared to low levels were associated with a higher risk of all-cause mortality (pooled HR $2.1395 \% \mathrm{Cl} 1.49-3.05$ ) [20]. Most previous studies observed a statistically significant association between preoperative IL6 and TNF $\alpha$ in relation to all-cause mortality $[14,17,19]$. In a meta-analysis including 6 studies with in total 679 CRC patients high versus low levels of IL6 were associated with a higher risk of all-cause mortality (pooled HR 1.76 95\% Cl 1.42-2.19) [17]. In a study in 119 CRC patients, a significant longer median survival time was observed in patients with high versus low levels of TNF $\alpha$ [19], while a small study in 74 CRC patients with local disease did not observe an association between TNF and mortality [44]. Regarding CRP, a meta-analysis including 21 studies with a total of 3934 CRC patients observed a pooled $\mathrm{HR}$ of $2.04(95 \% \mathrm{Cl} 1.45-2.86)$ for all-cause mortality, comparing high pre-operative CRP levels with low levels [7], which is in line with results of our study. Although we did not observe an association between IL10 and all-cause mortality, a previous study observed a worse survival rate with higher post-diagnostic IL10 levels [41]. Combining the results of our and previous studies, we can conclude that, in general, higher pre-operative and post-operative levels of pro-inflammatory markers are associated with worse CRC survival.

The mechanisms by which inflammation as reflected by aberrant expression of cytokines promotes CRC progression have extensively been studied in in-vitro and animal studies [5, 45]. Several interleukins, including IL6, IL8 and IL10, have tumour-promoting effects. Interleukin 6 has several important roles in cancer progression, as it stimulates processes such as cell proliferation, migration and angiogenesis. Interleukin-6 mainly exerts is tumour-promoting effects by activating signal transducer and activator of transcription 3 (STAT3) $[5,45]$. Interleukin-8 mainly promotes CRC progression through the activation of intercellular signaling phosphoinositide 3-kinases (PI3K) and Akt. The PI3k/Akt pathway plays an essential role in cell survival, migration and angiogenesis [45]. How IL10 is related to $\mathrm{CRC}$ progression is less clear. Some research suggest a tumour promoting effect, while other studies propose a tumour inhibitory effect $[18,45,46]$. In addition to interleukins, TNF $\alpha$ is also an important cytokine that mainly drives tumour growth by activating Nuclear Factor kappa B (NFKB) [5]. Finally, CRP plays important roles in inflammatory processes, apoptosis and the production of cytokines, particularly IL6 and TNF $\alpha$ [47], which in turn, stimulates CRC progression. Thus, all inflammatory markers investigated in the present study are mechanistically linked to CRC progression. Although IL6 and TNF $\alpha$ also seems to be associated with all-cause mortality, we observed the strongest associations for IL8 and hsCRP. One reason for this could be the variability in levels. The variation in plasma levels of IL6, IL10 and TNF $\alpha$ was low (median (IQR) 1.0 (0.7-1.6), 0.2 (0.2-0.3) and 2.1 (1.6-2.6), respectively), while more variation was observed in hSCRP and IL8 levels (median (IQR) 5.7 (4.3-8.1 and 2.5 (1.2-6.1), respectively). 
We also investigated whether lifestyle factors influenced the associations between levels of inflammatory markers and cancer outcomes. In our study only smoking status and BMI influenced the association between levels of inflammatory markers and cancer outcomes. Thus, although many of these lifestyle factors potentially influence both inflammation and survival [21-26], these lifestyle factors did not substantially influence the association between inflammation and recurrence and all-cause mortality in CRC patients in our study. One possible explanation for this could be that our participants were relatively health conscious, resulting in relatively low variation in dietary intake and physical activity levels.

An integrative evaluation of 1) systemic inflammation, 2) detailed profiling of inflammation and host immunity in the tumour microenvironment and 3) factors influencing inflammation is important to extend our knowledge on inflammation in relation to cancer recurrence and survival. This molecular pathological epidemiological approach $[42,43]$ is essential to further unravel such complex interrelationships. Thus as a next step, local inflammation at the tumour-site, the so-called tumour microenvironment, should be examined to further elucidate the role of inflammation in CRC recurrence and mortality. Special attention should be given to cytokines known to be involved in tumour progression such as IL6, IL8 and TNF $\alpha[5,45]$, and to immune cell infiltration [48] rather than on more general markers of inflammation, such as CRP. Finally, clinical and lifestyle factors should also be examined as they could potentially influence inflammation and could therefore modify the association between inflammation and CRC outcomes.

Our study has several strengths. First, we examined a set of cytokines suggested to play an important role in CRC prognosis before and after surgery. Furthermore, our study is one of the first studies that investigated the association between cytokines and CRC recurrence. In addition, previous studies assessed the association between inflammation markers and cancer outcomes using inflammation markers as a categorical exposure variable. However, it is debated whether categorization of a continuous variable is always appropriate [49]. In our study, we investigated inflammation markers as continuous variables. Besides, we also performed restricted cubic spline analyses to get more insight into the relationship between inflammation marker levels and cancer outcomes. Thus, results of our study extend existing evidence regarding the association between inflammation and CRC outcomes.

Our study also has some limitations. First, the absolute levels and the variation in levels of the inflammation markers were low. For example, the median IL6 levels was $1.0 \mathrm{pg} / \mathrm{ml}$ (IQR 0.7-1.6). The low variance in the data limit the ability to detect associations. Still, we observed statistically significant associations between several inflammation markers and cancer outcomes. Second, it would be of interest to investigate whether the association between inflammation markers and mortality is cancer specific or more related to other 
comorbid conditions such as cardiometabolic diseases. This could be of importance since chronic inflammation is also suggested to play a role in the onset of comorbidities such as cardiovascular diseases and diabetes [27, 28]. Unfortunately, we had no data available about the cause of death, due to strict privacy regulations in the Netherlands. Therefore, we were not able to perform analyses with disease-specific mortality as an outcome.

In conclusion, pre and post-operative levels of several inflammation markers were associated with an increased risk of recurrence and/or all-cause mortality in stage I-III CRC patients. The complex role of inflammation in cancer recurrence should be further elucidated using a molecular pathological epidemiology approach. 


\section{ADDITIONAL INFORMATION}

\section{FUNDING}

The COLON study was financially supported by Wereld Kanker Onderzoek Fonds, including funds from grant 2014/1179 as part of the World Cancer Research Fund International Regular Grant Programme; Alpe d'Huzes/Dutch Cancer Society (UM 2012-5653, UW 20135927, UW 2015-7946); and ERA-NET on Translational Cancer Research (TRANSCAN/Dutch Cancer Society (UW2013-6397, UW2014-6877) and the Netherlands Organization for Health Research and Development (ZonMw, the Netherlands). The EnCoRe study was supported by a grant from the Stichting Alpe d'HuZes within the research program 'Leven met kanker' of the Dutch Cancer Society (Grant No. UM-2010-4867) and by a grant from Kankeronderzoekfonds Limburg as part of Health Foundation Limburg (Grant No. 00005739).

\section{AUTHORS' CONTRIBUTIONS}

E Wesselink, MGJ Balvers, MJL Bours, MP Weijenberg, E Kampman, JHW de Wilt, RF Witkamp and FJB van Duijnhoven contributed to the design and conceptualization of this study. E Wesselink, DE Kok, RM Winkels, M van Zutphen, RWM Schrauwen, EA Kouwenhoven, ETP Keulen, SO Breukink and JHW de Wilt contributed to recruitment of participants and the data collection. Statistical data analyses were done by E Wesselink. Funding was obtained by FJB van Duijnhoven, E Kampman and MP Weijenberg. E. Kampman received the grants with numbers: 2014/1179, UW2013-6397 and UW20146877. F.J.B. van Duijnhoven received the grants with numbers: UW2013-5927 and UW2015-7946. M.P. Weijenberg received the grants with numbers: UM2010-4867, UM2012-2563, UM2014-6877 and 00005739. The manuscript was drafted by E Wesselink and FJB van Duijnhoven, and all authors critically read and revised the manuscript. All authors approved the final version of the manuscript.

\section{ACKNOWLEDGEMENTS}

The authors would like to thank the participants of the COLON study and the investigators at Wageningen University \& Research and the co-workers from the following hospitals for their involvement in recruitment for the COLON study: Hospital Gelderse Vallei, Ede; Radboudumc, Nijmegen; Slingeland Hospital, Doetinchem; Canisius Wilhelmina Hospital, Nijmegen; Rijnstate Hospital, Arnhem; Gelre Hospitals, Apeldoorn/Zutphen; Hospital Bernhoven, Uden; Isala, Zwolle; ZGT, Almelo; Martini Hospital, Groningen; Admiraal de Ruyter Hospital, Goes/Vlissingen. We would like to thank all participants of the EnCoRe study and the health professionals in the three hospitals involved in the recruitment of participants of the study: Maastricht University Medical Center, VieCuri Medical Center, and Zuyderland Medical Center. We would also like to thank the MEMIC center for data 
and information management for facilitating the logistic processes and data management of our study. Furthermore, we would like to thank the research dieticians and research assistant who are responsible for patient inclusion and follow-up, performing home visits, as well as data collection and processing. Finally, the authors would like to thank BEVITAL, Bergen, Norway for analyzing hsCRP in the COLON and EnCoRe study.

\section{DATA AVAILABILITY}

Since the data consist of identifying cohort information, some access restrictions apply and therefore cannot be made publicly available. Data will be shared with permission from the acting committee of the COLON Study. Requests for data can be sent to Dr.

Fränzel van Duijnhoven, Division of Human Nutrition and Health, Wageningen University \& Research, The Netherlands (e-mail: franzel.vanduijnhoven@wur.nl). Requests for data of the EnCoRe study can be sent to Dr. Martijn Bours, Department of Epidemiology, GROWSchool for Oncology and Developmental Biology, Maastricht University, the Netherlands (e-mail: m.bours@maastrichtuniversity.nl). 


\section{REFERENCE}

[1] Hanahan D, Weinberg RA. Hallmarks of cancer: the next generation. Cell. 2011;144:646-74.

[2] Lasry A, Zinger A, Ben-Neriah Y. Inflammatory networks underlying colorectal cancer. Nature immunology. 2016;17:230.

[3] Grivennikov SI, Greten FR, Karin M. Immunity, inflammation, and cancer. Cell. 2010;140:883-99.

[4] Shalapour S, Karin M. Immunity, inflammation, and cancer: an eternal fight between good and evil. The Journal of clinical investigation. 2015;125:3347-55.

[5] West NR, McCuaig S, Franchini F, Powrie F. Emerging cytokine networks in colorectal cancer. Nature Reviews Immunology. 2015;15:615-29.

[6] Coyle C, Cafferty FH, Langley RE. Aspirin and colorectal cancer prevention and treatment: is it for everyone? Current colorectal cancer reports. 2016;12:27-34.

[7] Woo HD, Kim K, Kim J. Association between preoperative C-reactive protein level and colorectal cancer survival: a meta-analysis. Cancer Causes \& Control. 2015;26:1661-70.

[8] Shrotriya S, Walsh D, Bennani-Baiti N, Thomas S, Lorton C. C-Reactive Protein Is an Important Biomarker for Prognosis Tumor Recurrence and Treatment Response in Adult Solid Tumors: A Systematic Review. PloS one. 2015;10:e0143080.

[9] Matsubara D, Arita T, Nakanishi M, Kuriu Y, Murayama Y, Kudou M, et al. The impact of postoperative inflammation on recurrence in patients with colorectal cancer. International Journal of Clinical Oncology. 2020;25:602-13.

[10] Guthrie GJK, Roxburgh CSD, Horgan PG, McMillan DC. Does interleukin-6 link explain the link between tumour necrosis, local and systemic inflammatory responses and outcome in patients with colorectal cancer? Cancer Treatment Reviews. 2013;39:89-96.

[11] Chang PH, Pan YP, Fan CW, Tseng WK, Huang JS, Wu TH, et al. Pretreatment serum interleukin-1 $\beta$, interleukin-6, and tumor necrosis factor- $\alpha$ levels predict the progression of colorectal cancer. Cancer medicine. 2016;5:426-33.

[12] Rasic I, Radovic S, Aksamija G. Relationship between chronic inflammation and the stage and histopathological size of colorectal carcinoma. Medical Archives. 2016;70:104.

[13] Szkaradkiewicz A, Marciniak R, Chudzicka-Strugała I, Wasilewska A, Drews M, Majewski P, et al. Proinflammatory cytokines and IL-10 in inflammatory bowel disease and colorectal cancer patients. Archivum immunologiae et therapiae experimentalis. 2009;57:291.

[14] Knupfer H, Preiss R. Serum interleukin-6 levels in colorectal cancer patients--a summary of published results. International journal of colorectal disease. 2010;25:135-40.

[15] Bălăşoiu M, Bălăşoiu AT, Mogoantă SŞ, Bărbălan A, Stepan AE, Ciurea RN, et al. Serum and tumor microenvironment IL-8 values in different stages of colorectal cancer. Rom J Morphol Embryol. 2014;55:575-8.

[16] Seruga B, Zhang H, Bernstein LJ, Tannock IF. Cytokines and their relationship to the symptoms and outcome of cancer. Nature Reviews Cancer. 2008;8:887.

[17] Xu J, Ye Y, Zhang H, Szmitkowski M, Mäkinen M, Li P, et al. Diagnostic and prognostic value of serum interleukin-6 in colorectal cancer. Medicine. 2016;95.

[18] Abtahi S, Davani F, Mojtahedi Z, Hosseini SV, Bananzadeh A, Ghaderi A. Dual association of serum interleukin-10 levels with colorectal cancer. Journal of cancer research and therapeutics. 2017;13:252. [19] Stanilov N, Miteva L, Dobreva Z, Stanilova S. Colorectal cancer severity and survival in correlation with tumour necrosis factor-alpha. Biotechnology \& Biotechnological Equipment. 2014;28:911-7.

[20] Xia W, Chen W, Zhang Z, Wu D, Wu P, Chen Z, et al. Prognostic value, clinicopathologic features and diagnostic accuracy of interleukin-8 in colorectal cancer: a meta-analysis. PloS one. 2015;10:e0123484.

[21] Wedell-Neergaard A-S, Eriksen L, Grønbæk M, Pedersen BK, Krogh-Madsen R, Tolstrup J. Low fitness is associated with abdominal adiposity and low-grade inflammation independent of BMI. PloS one. 2018;13.

[22] Calder PC, Ahluwalia N, Brouns F, Buetler T, Clement K, Cunningham K, et al. Dietary factors and low-grade inflammation in relation to overweight and obesity. British Journal of Nutrition. 2011;106:S1-S78. 
[23] Yanbaeva DG, Dentener MA, Creutzberg EC, Wesseling G, Wouters EF. Systemic effects of smoking. Chest. 2007;131:1557-66.

[24] Meyerhardt JA, Giovannucci EL, Holmes MD, Chan AT, Chan JA, Colditz GA, et al. Physical activity and survival after colorectal cancer diagnosis. Journal of clinical oncology. 2006;24:3527-34.

[25] Meyerhardt JA, Niedzwiecki D, Hollis D, Saltz LB, Hu FB, Mayer RJ, et al. Association of dietary patterns with cancer recurrence and survival in patients with stage III colon cancer. JAMA : the journal of the American Medical Association. 2007;298:754-64.

[26] Pelser C, Arem H, Pfeiffer RM, Elena JW, Alfano CM, Hollenbeck AR, et al. Prediagnostic lifestyle factors and survival after colon and rectal cancer diagnosis in the National Institutes of Health (NIH)-AARP Diet and Health Study. Cancer. 2014;120:1540-7.

[27] Alfano CM, Peng J, Andridge RR, Lindgren ME, Povoski SP, Lipari AM, et al. Inflammatory cytokines and comorbidity development in breast cancer survivors versus noncancer controls: evidence for accelerated aging? Journal of Clinical Oncology. 2017;35:149.

[28] Lopez-Candales A, Burgos PMH, Hernandez-Suarez DF, Harris D. Linking chronic inflammation with cardiovascular disease: from normal aging to the metabolic syndrome. Journal of nature and science. 2017;3. [29] van Harten-Gerritsen AS, Balvers MG, Witkamp RF, Kampman E, van Duijnhoven FJ. Vitamin D, inflammation and colorectal cancer progression: A review of mechanistic studies and future directions for epidemiological studies. Cancer Epidemiology and Prevention Biomarkers. 2015:cebp. 0601.2015.

[30] Wesselink E, Balvers M, Bours MJL, de Wilt JHW, Witkamp RF, van Baar H, et al. The association between circulating levels of vitamin $D$ and inflammatory markers in the first 2 years after colorectal cancer diagnosis. Therapeutic Advances in Gastroenterology. 2020;13:1756284820923922.

[31] Winkels RM, Heine-Broring RC, van Zutphen M, van Harten-Gerritsen S, Kok DE, van Duijnhoven FJ, et al. The COLON study: Colorectal cancer: Longitudinal, Observational study on Nutritional and lifestyle factors that may influence colorectal tumour recurrence, survival and quality of life. BMC Cancer. 2014;14:374.

[32] van Roekel EH, Bours MJ, de Brouwer CP, Ten Napel H, Sanduleanu S, Beets GL, et al. The Applicability of the International Classification of Functioning, Disability, and Health to Study Lifestyle and Quality of Life of Colorectal Cancer Survivors. Cancer epidemiology, biomarkers \& prevention : a publication of the American Association for Cancer Research, cosponsored by the American Society of Preventive Oncology. 2014;23:1394405.

[33] Meyer K, Ueland PM. Targeted quantification of C-reactive protein and cystatin c and its variants by immuno-MALDI-MS. Analytical chemistry. 2014;86:5807-14.

[34] Hopkins MH, Owen J, Ahearn T, Fedirko V, Flanders WD, Jones DP, et al. Effects of supplemental vitamin D and calcium on biomarkers of inflammation in colorectal adenoma patients: a randomized, controlled clinical trial. Cancer prevention research. 2011;4:1645-54.

[35] Wendel-Vos GC, Schuit AJ, Saris WH, Kromhout D. Reproducibility and relative validity of the short questionnaire to assess health-enhancing physical activity. Journal of clinical epidemiology. 2003;56:1163-9.

[36] Van Leersum N, Snijders H, Henneman D, Kolfschoten N, Gooiker G, Ten Berge M, et al. The Dutch surgical colorectal audit. European Journal of Surgical Oncology (EJSO). 2013;39:1063-70.

[37] Khoo AL, Chai L, Koenen H, Sweep F, Joosten I, Netea M, et al. Regulation of cytokine responses by seasonality of vitamin D status in healthy individuals. Clinical \& Experimental Immunology. 2011;164:72-9.

[38] de Jager W, Bourcier K, Rijkers GT, Prakken BJ, Seyfert-Margolis V. Prerequisites for cytokine measurements in clinical trials with multiplex immunoassays. BMC immunology. 2009;10:52.

[39] Takasu C, Shimada M, Kurita N, Iwata T, Nishioka M, Morimoto S, et al. Impact of C-reactive protein on prognosis of patients with colorectal carcinoma. Hepato-gastroenterology. 2013;60:507-11.

[40] Hara M, Nagasaki T, Shiga K, Takahashi H, Takeyama H. High serum levels of interleukin- 6 in patients with advanced or metastatic colorectal cancer: the effect on the outcome and the response to chemotherapy plus bevacizumab. Surgery Today. 2017;47:483-9.

[41] Li B, Wang F, Ma C, Hao T, Geng L, Jiang H. Predictive value of IL-18 and IL-10 in the prognosis of patients with colorectal cancer. Oncology letters. 2019;18:713-9. 
[42] Ogino S, Campbell PT, Nishihara R, Phipps Al, Beck AH, Sherman ME, et al. Proceedings of the second international molecular pathological epidemiology (MPE) meeting. Cancer Causes \& Control. 2015;26:959-72.

[43] Ogino S, Chan AT, Fuchs CS, Giovannucci E. Molecular pathological epidemiology of colorectal neoplasia: an emerging transdisciplinary and interdisciplinary field. Gut. 2011;60:397-411.

[44] Nikiteas NI, Tzanakis N, Gazouli M, Rallis G, Daniilidis K, Theodoropoulos G, et al. Serum IL-6, TNFalpha and CRP levels in Greek colorectal cancer patients: prognostic implications. World journal of gastroenterology : WJG. 2005;11:1639-43.

[45] Li J, Huang L, Zhao H, Yan Y, Lu J. The Role of Interleukins in Colorectal Cancer. International Journal of Biological Sciences. 2020;16:2323-39.

[46] Mannino MH, Zhu Z, Xiao H, Bai Q, Wakefield MR, Fang Y. The paradoxical role of IL-10 in immunity and cancer. Cancer letters. 2015;367:103-7.

[47] Sproston NR, Ashworth JJ. Role of C-Reactive Protein at Sites of Inflammation and Infection. Frontiers in immunology. 2018;9:754.

[48] Pagès F, Mlecnik B, Marliot F, Bindea G, Ou F-S, Bifulco C, et al. International validation of the consensus Immunoscore for the classification of colon cancer: a prognostic and accuracy study. The Lancet. 2018;391:212839.

[49] Bennette C, Vickers A. Against quantiles: categorization of continuous variables in epidemiologic research, and its discontents. BMC Medical Research Methodology. 2012;12:21. 


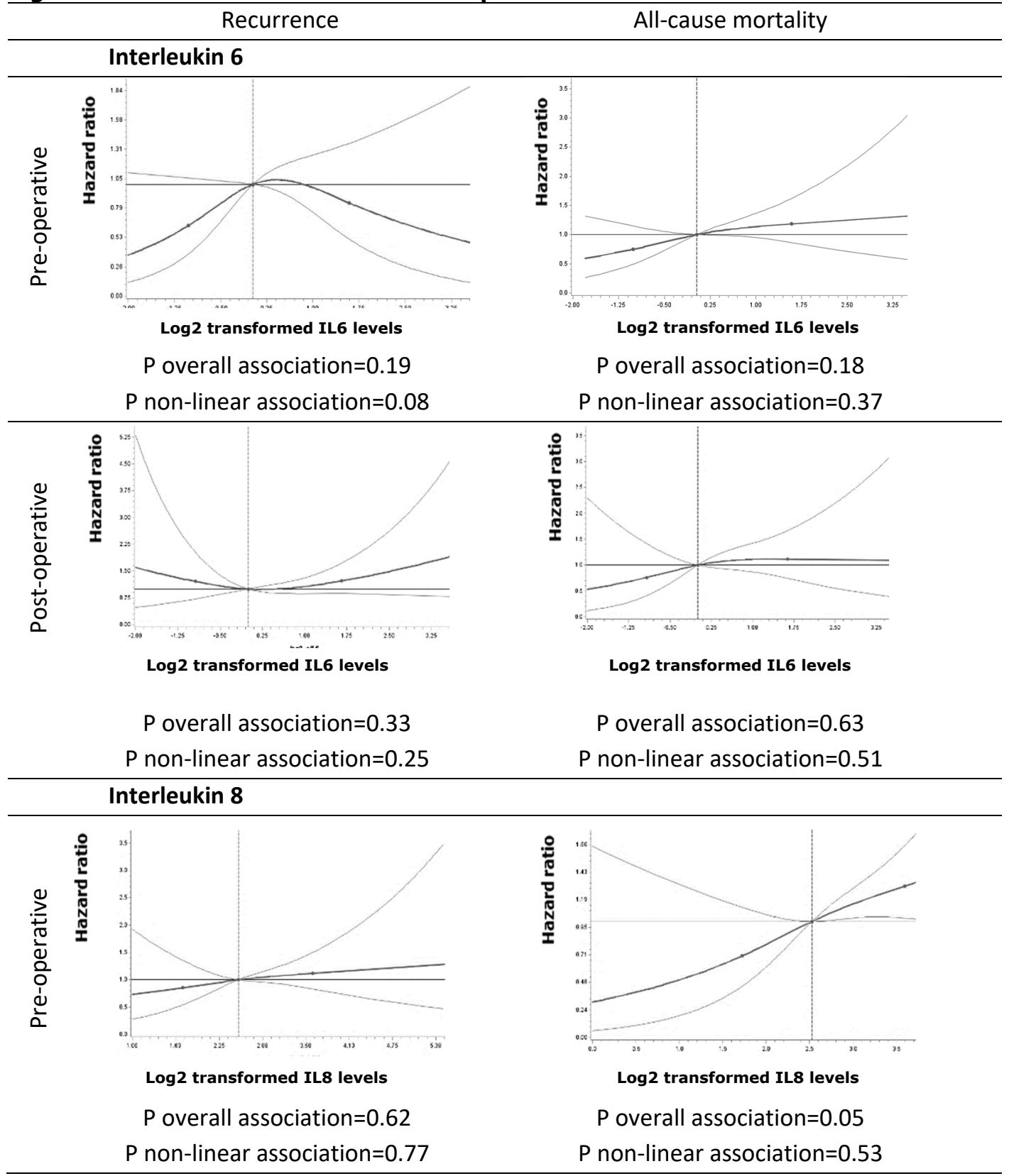




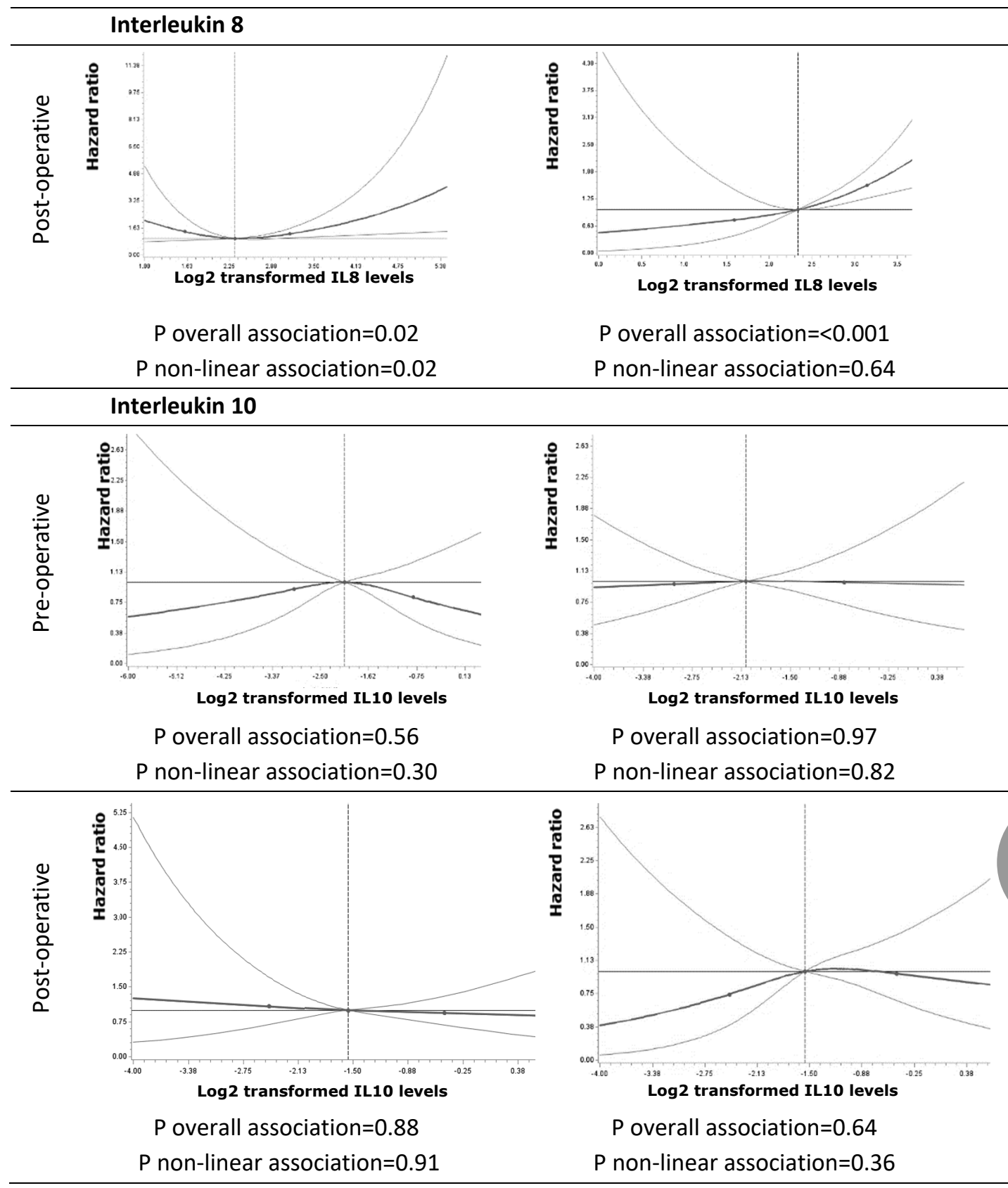




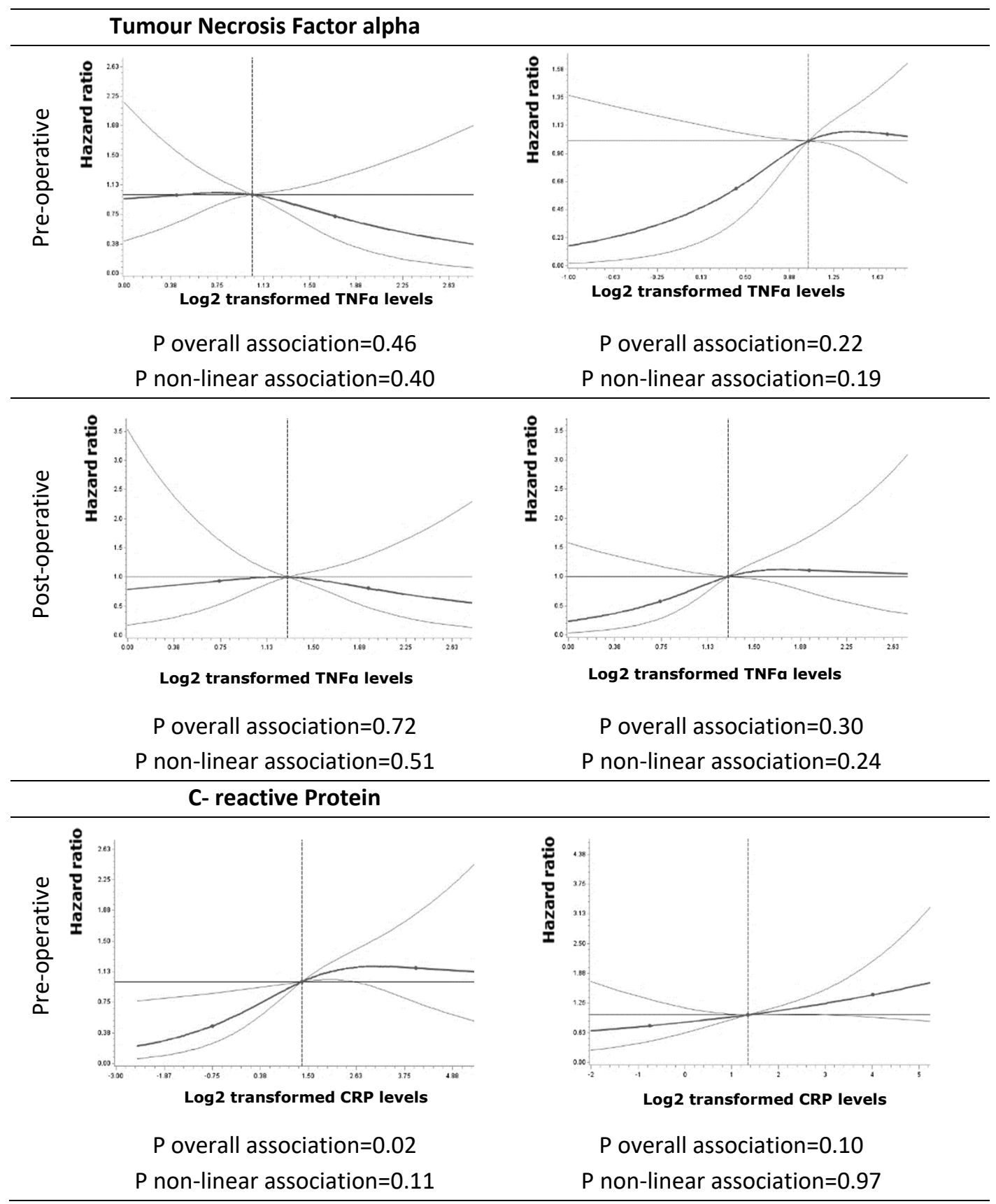




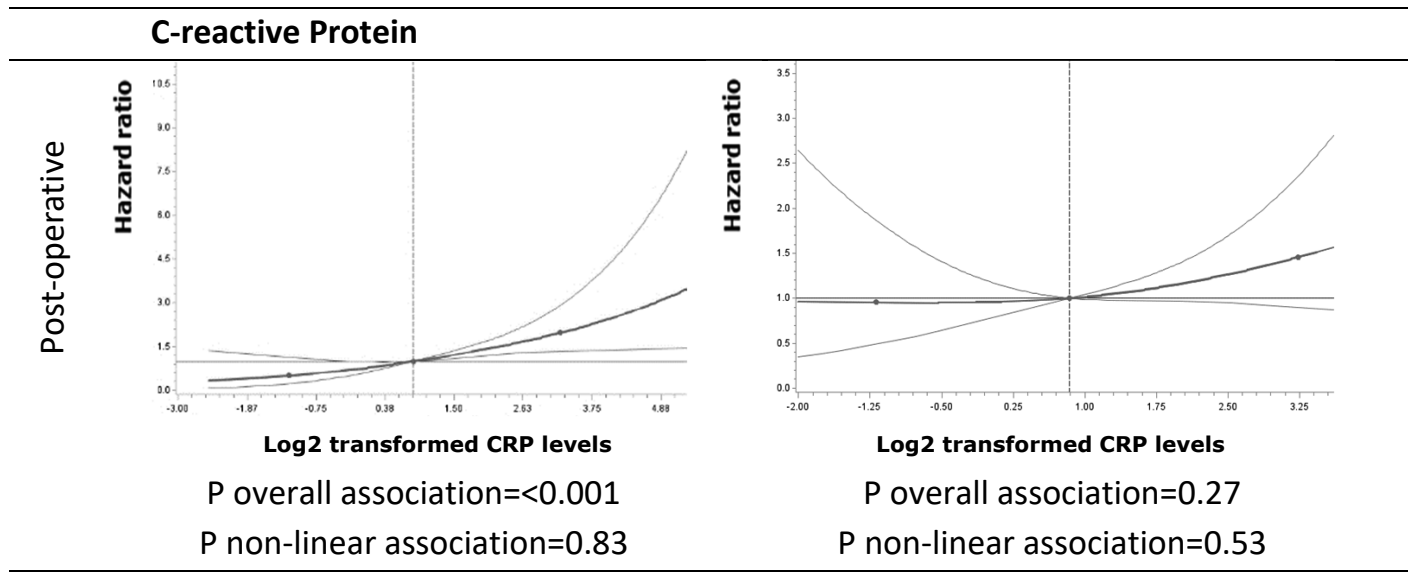

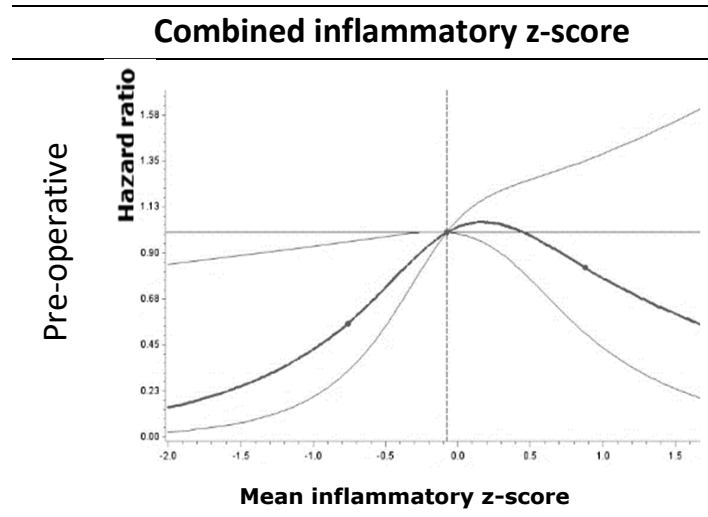

P overall association $=0.10$

$\mathrm{P}$ non-linear association $=0.05$

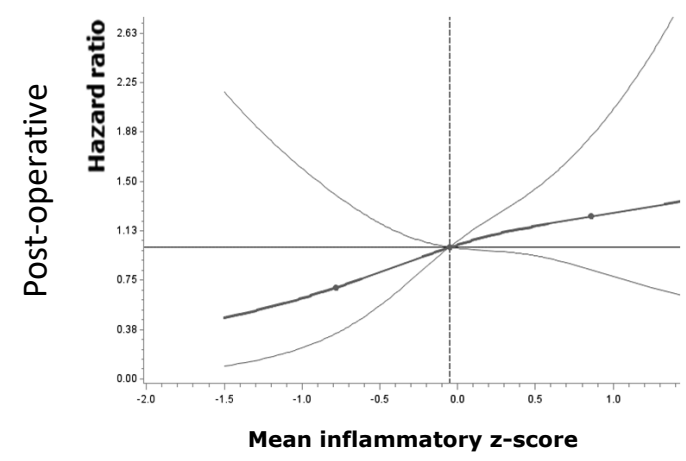

P overall association $=0.33$

$P$ non-linear association $=0.62$

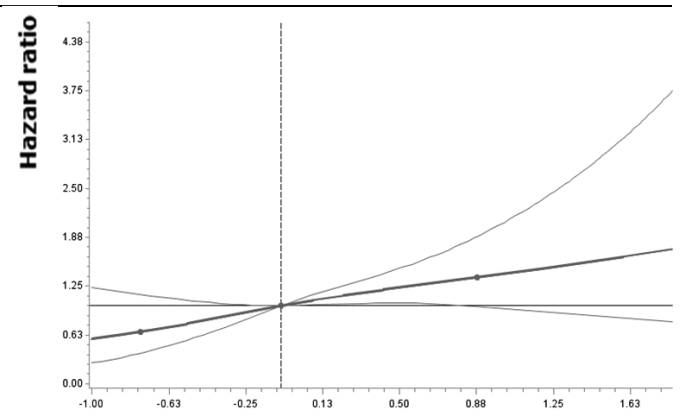

Mean inflammatory z-score

P overall association $=0.05$

$\mathrm{P}$ non-linear association $=0.55$

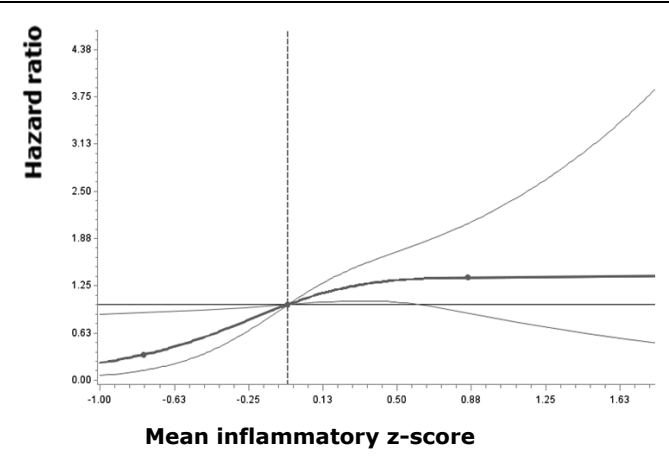

$P$ overall association $=0.03$

$\mathrm{P}$ non-linear association $=0.10$

* Models were adjusted for age, sex, BMI, stage of disease, smoking status, cohort and season of blood collection. Knots were placed on the $10^{\text {th }}, 50^{\text {th }}$ and $90^{\text {th }}$ percentile, where the $50^{\text {th }}$ percentile was used as the reference. Dark grey line represents the effect estimation; light grey lines the lower and upper $95 \%$ confidence intervals. 
TABEL

Table S1: Baseline characteristics of colorectal cancer patients stratified for changes in levels of inflammation markers in the first six months after diagnosis.

\begin{tabular}{|c|c|c|c|}
\hline & $\begin{array}{l}\text { Increased levels (>1 SD) } \\
\qquad(N=76)\end{array}$ & $\begin{array}{c}\text { Stable } \\
(\mathrm{N}=455)\end{array}$ & $\begin{array}{l}\text { Decreased levels (>1 SD) } \\
\qquad(\mathrm{N}=\mathbf{7 2})\end{array}$ \\
\hline Age (years) & $67.0(61.0-70.3)$ & $66.6(61.7-72.7)$ & $68.5(63-74)$ \\
\hline Gender (female) & $18(24)$ & $158(35)$ & $22(31)$ \\
\hline BMI $\left(\mathrm{kg} / \mathrm{m}^{2}\right)$ & $27.7(24.6-30.5)$ & $27.2(24.6-29.9)$ & $25.8(23.2-27.9)$ \\
\hline Unknown & 0 & 1 & 0 \\
\hline \multicolumn{4}{|l|}{ Education ${ }^{\text {b }}$} \\
\hline Low & $38(50)$ & $213(48)$ & $28(40)$ \\
\hline Medium & $22(29)$ & $116(26)$ & $17(24)$ \\
\hline High & $16(21)$ & $113(26)$ & $25(36)$ \\
\hline Unknown & 0 & 13 & 2 \\
\hline \multicolumn{4}{|l|}{ Smoking habits } \\
\hline Current & $9(12)$ & $52(12)$ & $7(10)$ \\
\hline Former & $45(60)$ & $259(57)$ & $45(63)$ \\
\hline Never & $22(29)$ & $141(31)$ & $20(28)$ \\
\hline Unknown & 0 & 3 & 0 \\
\hline \multicolumn{4}{|l|}{ Physical activity $^{c}$} \\
\hline (hours/week) & $9.3(4.5-22.3)$ & $12.0(5.0-20.0)$ & $10.0(3.8-18.9)$ \\
\hline unknown & 0 & 3 & 0 \\
\hline \multicolumn{4}{|l|}{ Dietary factors } \\
\hline Fruits & $121(69-256)$ & $152(66-227)$ & $105(43-213)$ \\
\hline Vegetables & $103(76-170)$ & $115(73-173)$ & $114(69-165)$ \\
\hline Fibre & $22(18-28)$ & $22(17-27)$ & $18(15-23)$ \\
\hline Red meat & $49(35-70)$ & $41(25-59)$ & $37(22-53)$ \\
\hline Processed meat & $31(19-50)$ & $30(13-47)$ & $30(10-43)$ \\
\hline Alcohol & $12(2-25)$ & $9(1-20)$ & $4(1-24)$ \\
\hline Unknown & 0 & 7 & 1 \\
\hline \multicolumn{4}{|l|}{ Type of cancer } \\
\hline Colon & $45(59)$ & $299(66)$ & $57(79)$ \\
\hline Rectum & $31(41)$ & $156(34)$ & $15(21)$ \\
\hline \multicolumn{4}{|l|}{ Tumour stage } \\
\hline 1 & $16(21)$ & $146(32)$ & $13(18)$ \\
\hline II & $14(18)$ & $123(27)$ & $37(51)$ \\
\hline III & $46(61)$ & $186(41)$ & $22(31)$ \\
\hline \multicolumn{4}{|l|}{ Type of treatment } \\
\hline Only surgery & $34(45)$ & $262(58)$ & $41(57)$ \\
\hline Surgery and chemotherapy & $20(26)$ & $89(20)$ & $18(25)$ \\
\hline Surgery and radiotherapy & $9(12)$ & $54(12)$ & $10(14)$ \\
\hline Surgery and chemo radiation & $11(15)$ & $42(9)$ & $3(4)$ \\
\hline Unknown & 2 & 8 & 0 \\
\hline Use of NSAIDs (yes) & $9(12)$ & $87(19)$ & $19(26)$ \\
\hline
\end{tabular}




\begin{tabular}{|c|c|c|c|}
\hline Comorbidities & & & \\
\hline Yes & $54(71)$ & $345(76)$ & $50(70)$ \\
\hline Unknown & 0 & 1 & 0 \\
\hline $\begin{array}{c}\text { Serum } 25(\mathrm{OH}) \mathrm{D}_{3} \text { levels } \\
(\mathrm{nmol} / \mathrm{L})\end{array}$ & $51.8(36.7-78.0)$ & $53.2(41.0-68.2)$ & $50(36-65)$ \\
\hline \multicolumn{4}{|l|}{ Season of blood collection ${ }^{a}$} \\
\hline \multicolumn{4}{|l|}{ Spring } \\
\hline Summer & $18(24)$ & $138(30)$ & $22(31)$ \\
\hline Autumn & $30(39)$ & $117(26)$ & $19(26)$ \\
\hline \multirow[t]{2}{*}{ Winter } & $11(15)$ & $87(19)$ & $14(19)$ \\
\hline & $17(22)$ & $113(25)$ & $17(24)$ \\
\hline \multicolumn{4}{|l|}{ Inflammatory markers } \\
\hline IL6 (pg/mL) & $0.8(0.6-1.2)$ & $0.9(0.7-1.4)$ & $2.2(1.3-4.3)$ \\
\hline IL8 (pg/mL) & $5.1(3.7-6.8)$ & $5.5(4.2-7.8)$ & $9.0(5.9-14.8)$ \\
\hline IL10 (pg/mL) & $0.2(0.1-0.3)$ & $0.2(0.2-0.3)$ & $0.4(0.2-3.4)$ \\
\hline TNF (pg/mL) & $1.9(1.5-2.4)$ & $2.0(1.6-2.5)$ & $2.7(2.1-3.4)$ \\
\hline CRP $(\mu \mathrm{g} / \mathrm{mL})$ & $1.6(0.8-3.5)$ & $2.4(1.1-4.8)$ & $13.7(4.7-31.3)$ \\
\hline \multicolumn{4}{|l|}{ Cohort } \\
\hline COLON & $45(59)$ & $310(68)$ & $55(76)$ \\
\hline EnCoRe & $31(41)$ & $145(32)$ & $17(24)$ \\
\hline Death & $13(17)$ & $42(9)$ & $5(7)$ \\
\hline Recurrence & $16(21)$ & $48(11)$ & $5(7)$ \\
\hline
\end{tabular}

Values presented are median (quartile 1 - quartile 3) or number (percentages). Abbreviations: 25(OH) D3, 25hydroxyvitamin D3; NSAIDs, non-steroid anti-inflammatory drugs. *Low, moderate and high levels of inflammation were derived from the tertiles of the combined inflammatory-z-scores of IL6 + IL8 + IL10 + TNF $\alpha+$ CRP /5. For 81 patients, levels of one inflammation marker was missing for those patients the main inflammatory $z$-score was calculated by; summing the z-scores of the four available inflammation markers /4. For 5 patients more than one inflammatory marker was missing, for these patients no mean inflammatory z-score was calculated. Cut-off points for the tertiles of the total inflammation level were: -0.32 and 0.18 .

a: Low education was defined as primary school and lower general secondary education; medium as lower vocational training and higher general secondary education; high as high vocational training and university. b: Activities with a Metabolic Equivalent score (MET score) $\geq 3$ were defined as moderate to vigorous physical activity

c: Intake in grams per day.

d: Spring: March-May; Summer: June-August; Autumn: September-November; Winter: December-February. 



\title{
CHAPTER 7
}

\section{THE ASSOCIATION BETWEEN THE ADAPTED DIETARY INFLAMMATORY INDEX AND COLORECTAL CANCER RECURRENCE AND ALL-CAUSE MORTALITY}

\author{
Evertine Wesselink \\ Laura E. Staritsky \\ Moniek van Zutphen \\ Anne J.M.R. Geijsen \\ Dieuwertje E. Kok \\ Flip M. Kruyt \\ Renzo P. Veenstra \\ Ernst Jan Spillenaar Bilgen \\ Ewoud A. Kouwenhoven \\ Johannes H.W. de Wilt \\ Ellen Kampman
}

Franzel J.B. van Duijnhoven

Clinical Nutrition. 2021 Jan 11;S0261-5614(21)00010-8. 


\section{ABSTRACT}

Background \& aim: The inflammatory potential of the diet has been linked to colorectal cancer (CRC) development and mortality. However, it is unknown whether it is also associated with $\mathrm{CRC}$ recurrence. Therefore, the aim of this study was to investigate the associations between the inflammatory potential of the diet and plasma inflammation markers as well as recurrence and all-cause mortality in CRC patients.

Methods: Data of the Colorectal cancer, Observational, LONgitudinal (COLON) study, a prospective cohort study, was used. Dietary intake, assessed using a semi-quantitative food frequency questionnaire, was available for 1,478 patients at diagnosis and for 1,334 patients six months after diagnosis. Dietary intake data were used to calculate the adapted dietary inflammatory index (ADII). Data about cancer recurrence and all-cause mortality, were assessed through linkage with the Netherlands Cancer Registry and the Municipal Personal Records Database, respectively. The association between the ADII (continuous) and inflammation markers (Interleukin (IL)6, IL8, IL10, Tumour Necrosis Factor(TNF) $\alpha$, high sensitivity C-reactive protein (hsCRP) and a summary inflammatory zscore), measured with a multiplex assay using electrochemiluminiscence detection, was assessed using quantile regression analyses. Restricted cubic splines (RCS) analyses and multivariable Cox proportional hazard models were used to explore the relationship between the ADII and CRC outcomes.

Results: During a median follow-up time of 3.2 years (Interquartile range (IQR) 2.0-4.1) for recurrence and 4.8 years (IQR 3.5-5.9) for all-cause mortality, 228 recurrences and 279 deaths occurred. A more pro-inflammatory diet at diagnosis as well as six months after diagnosis was associated with higher levels of TNF $\alpha$, hSCRP and the summary inflammatory z-score. Results of RCS showed no relationship between the ADII and CRC outcomes at both time points. Also results of the Cox proportional hazard models showed no associations between the ADII at both time points and recurrence $(\mathrm{HR}(95 \% \mathrm{Cl}) 0.98$ (0.94-1.04) \& $0.96(0.91-1.02)$ or all-cause mortality (HR $(95 \% \mathrm{Cl}) 1.03(0.98-1.07) \& 1.00$ (0.95-1.05)).

Conclusion: Our study did not show an association between the ADII and recurrence and all-cause mortality in CRC patients. Further research should also take into account molecular tumour subtypes, as the effect of the inflammatory potential of the diet on cancer recurrence and mortality is more likely to be present in tumours with an inflammatory signature.

Keywords: inflammatory potential of the diet, inflammatory markers, colorectal cancer, recurrence, all-cause mortality, 


\section{INTRODUCTION}

Chronic low-grade inflammation is related to the development of several chronic diseases, including cancer [1]. In general, acute inflammation is a useful process and necessary for repair [2]. However, chronic inflammation can interfere with normal homeostasis as it contributes to, among others, excessive cell proliferation and DNA damage [1, 3]. Moreover, higher levels of inflammation markers, including C-reactive protein (CRP), Interleukin (IL)6, IL8, IL10 and tumour necrosis factor (TNF) $\alpha$, have been associated with a worse progression free-, cancer specific- and overall survival [4-11] and a higher risk of CRC recurrence [12]. Diet has a marked effect on inflammation and subsequent health status [13]. A high intake of red and processed meat and a low intake of fruits and vegetables has been linked to cancer development and progression via molecular processes that induce chronic inflammation [13]. Besides, an excess in dietary intake, leading to a higher BMI, also induces chronic inflammation [13]. Overweight and obesity in turn are linked to cancer development and progression [14, 15].

Over the years, several indices have been developed to indicate the inflammatory potential of the diet of an individual. The Dietary Inflammatory Index (DII) was first established in 2009 [16] by the University of South Carolina`s Cancer Prevention and Control program and updated in 2014 [17]. Also an energy-adjusted DII (E-DII) was developed by that same group $[18,19]$. This index and other indices, such as the Adapted Dietary Inflammatory Index (ADII) [20], developed by other groups, are based on studies that investigated foods and nutrients in relation to inflammatory markers, such as cytokines. The inflammatory potential of each food or nutrient is determined by its ability to either increase or decrease inflammatory markers, including CRP, IL1 $\beta$, IL4, IL6, IL10 or TNF $\alpha[17,20]$.

Previous studies have shown that a higher inflammatory potential of the diet is linked to a higher CRC risk, whereas a lower dietary inflammatory potential relates to a lower CRC risk $[21,22]$. However, only limited studies investigated the association between the inflammatory potential of the diet and clinical outcomes in CRC patients. The studies by Galas et al. showed that the DII assessed at diagnosis can be used as an indicator for longer hospitalization [23] and survival in CRC patients without distant metastasis [24]. Three recent studies in CRC survivors observed a higher risk of all-cause mortality with a more pro-inflammatory diet pre and post diagnosis [25-27]. The association between the inflammatory potential of the diet and CRC recurrence, however, has not been studied yet.

The objective of this study was to investigate whether the adapted dietary inflammatory index, measured at and post diagnosis, is associated with the inflammatory markers IL6, IL8, IL10, TNF $\alpha$ and CRP, as well as with CRC recurrence and all-cause mortality. We expected a more pro-inflammatory potential of the diet to be associated with higher 
levels of IL6, IL8, TNF $\alpha$ and CRP and lower levels of IL10. In addition, we expected a more pro-inflammatory diet to be associated with a higher risk of $\mathrm{CRC}$ recurrence and all-cause mortality.

\section{MATERIALS AND METHODS}

\section{STUDY POPULATION}

In total, 1,766 CRC patients were recruited between August 2010 and December 2017 (Figure 1) in the Colorectal cancer: Longitudinal, Observational study, on Nutritional and lifestyle factors that may influence CRC tumour recurrence, survival and quality of life COLON- study. The design of the COLON study (NCT03191110; ClinicalTrials.gov) has been described earlier [28]. In short, newly diagnosed CRC patients were recruited directly after diagnosis in 11 hospitals in the Netherlands and were followed during and after treatment. Men and women above the age of 18 were eligible. Non-Dutch speaking patients, patients with a history of CRC, (partial) bowel resection, chronic inflammatory bowel disease, hereditary CRC syndromes (e.g. Lynch syndrome, Familial Adenomatous Polyposis, Peutz-Jegher), dementia or another mental condition obstructing participation were excluded from the study. The COLON study was approved by the Committee on Research involving Human Subjects, region Arnhem-Nijmegen, the Netherlands (2009349). All patients signed informed consent.

Patients with missing data on dietary intake at diagnosis ( $\mathrm{N}=288)$, missing data on recurrence $(n=84)$ were excluded leaving 1478 patients for the analyses regarding mortality, and 1394 for the analyses regarding recurrence (Figure 1). Only samples stored for 2 years or less were analysed, since, cytokines were previously shown to remain stable in plasma for a period up to 2 years of storage at $-80^{\circ} \mathrm{C}$ [29]. Therefore, data on inflammation markers were not available for 954 patients, leaving 524 patients for the analyses regarding inflammation markers. Twenty-five patients died in the first six months after diagnosis, 14 had a recurrence and for 119 no dietary data was available. In total, 1334,1242 and 450 patients were included in the analyses regarding mortality, recurrence and inflammation markers six months after diagnosis, respectively. 


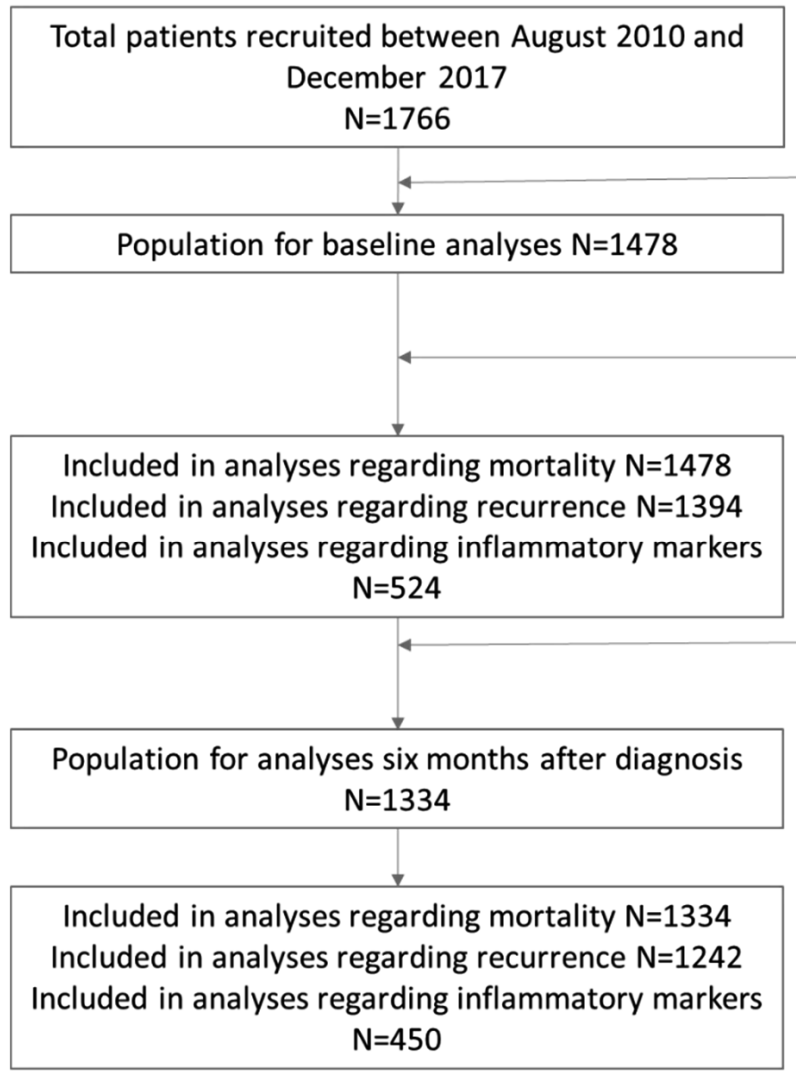

Missing data on dietary intake at diagnosis $(\mathrm{N}=288)$

Missing data on recurrence $\mathrm{N}=84$

Missing data on inflammation markers $\mathrm{N}=954$

Died in first six months $(\mathrm{N}=25)$

Recurrence in first six months $(\mathrm{N}=14)$

No dietary data available $(\mathrm{N}=119)$

Figure 1 Flowchart representing patient selection for the current study.

\section{COLLECTION OF DIETARY DATA AND CALCULATION OF THE ADII}

Habitual dietary intake was assessed at diagnosis as well as six months after diagnosis using a 204-item semi-quantitative food frequency questionnaire (FFQ). The reference period for the FFQ was the month before diagnosis or the previous month during followup. Dietary intake of nutrients was calculated for each food item based on frequency of intake, number of portions and portion size, as well as the type of product. Average daily intakes of nutrients were calculated using the 2011 Dutch food composition table (NEVO) [30]. The food components that were included in the calculation of the ADII were: protein, saturated fatty acids (SFAs), mono unsaturated fatty acids (MUFAs), trans fatty acids, n-3 poly unsaturated fatty acids (PUFAs), n-6 PUFAs, cholesterol, carbohydrate, fibre, alcohol, caffeine, vitamin A, $\beta$-carotene, thiamine, riboflavin, niacin, vitamin B6, folate, vitamin $B 12$, vitamin $C$, vitamin $D$, vitamin $E$, iron, magnesium, selenium, zinc, tea and quercetin. The NEVO did not provide information on the caffeine content of food items. Therefore, the estimated caffeine content of $68 \mathrm{mg} / 100 \mathrm{~mL}$ for coffee and $20 \mathrm{mg} / 100 \mathrm{~mL}$ for tea by 
van Woudenbergh et al. was used [20]. No data was available on type of soft drinks, caffeine content of soft drinks is thus not included in the calculation for caffeine.

Several steps were conducted to calculate the ADII score. First, energy adjustment for each nutrient was done using the residual method [31]. Second, to avoid that the variation in the ADII was solely driven by a few dietary components with a large range in intake, we standardized the individual nutrient intake. Standardization was done by calculating zscores, which was done by dividing the energy-adjusted intake by the standard deviation of the study population. The standardized energy-adjusted intake was then multiplied by the inflammatory weight score of a specific nutrient (derived from Shivappa et al., 2014; [17]). When the intake of alcohol was above $40 \mathrm{~g} /$ day, it was assumed not likely to have an anti-inflammatory effect [20]. Therefore, in these cases an Inflammatory Weight of zero was assigned to alcohol [20]. Finally, all nutrient-specific ADIl scores were summed to create the overall ADII score for an individual. Positive ADII scores are considered proinflammatory and negative ADII scores are considered anti-inflammatory. The contribution of each nutrient to the ADII score for both time points, assessed by using forward linear regression, is shown in supplementary data Table S1. To get more insights in food-groups contributing to either an anti- or pro-inflammatory potential of the diet the median intake of 39 previously defined food groups [32] were compared between tertiles of the ADII scores (Table S2).

\section{BLOOD COLLECTION AND INFLAMMATION MARKERS}

Blood samples were obtained during a regular clinical visit in the hospital at time of diagnosis and six months after diagnosis. All blood samples in EDTA tubes were centrifuged and aliquoted into plasma and were stored in a freezer at $-80^{\circ} \mathrm{C}$ until further analysis.

Plasma levels of IL6, IL8, IL10 and TNF $\alpha$ were determined using a custom-made multiplex assay using electrochemiluminiscence detection (Meso Scale Diagnostics, Rockville, Maryland, USA). The analyses were performed following the manufacturers' instructions, and assay plates were analysed on a QuickPlex SQ 120 plate reader (Meso Scale Diagnostics). Only samples stored $<2$ years were analysed [29], resulting in a subset of 524 samples at diagnosis and 450 six months after diagnosis. The highest inter and intrabatch coefficients of variation for all cytokines were $<8 \%$, and reported values deviated no more than $15 \%$ from the assigned target values.

High sensitivity C-reactive protein (hSCRP) was measured using an immuno-MALDI mass spectrometry method [33] (BEVITAL, Bergen, Norway). The inter-assay coefficient ranged between 3-6\%. 
Also a combined summary inflammatory z-score for inflammation markers was calculated to cluster conceptually related markers of low-grade inflammation and improve statistical efficiency. This was done by summing the z-scores of each inflammatory marker (inflammatory z-score $=$

$\left.\frac{-z_{\text {Score }}(\text { LnIL10 })+z_{\text {score }}(\text { LnIL8 })+z_{\text {score }}(\operatorname{LnIL6})+z_{\text {score }}(\operatorname{LnTNF\alpha })+z_{\text {score }}(\operatorname{LnCRP})}{\text { number of cytokines }}\right)$. The z-score for IL-10 was subtracted as this is a known anti-inflammatory cytokine [34].

\section{CRC RECURRENCE AND ALL-CAUSE MORTALITY}

Information on recurrence was collected from medical records by the Dutch Cancer Registry. Recurrence was defined as a locoregional recurrence or distant metastasis. Information on all-cause mortality was gathered from linkage with the Municipal Personal Record Database.

Follow-up time for recurrence was calculated starting from the date of dietary assessment until date of recurrence or until the date recurrence status was updated (February 2018) or the date of end of follow-up, whichever came first. For all-cause mortality follow-up time was defined starting from the date of dietary assessment until date of death, or until the last date survival status was updated (December 2019), or the date of end of followup, whichever came first.

\section{DEMOGRAPHICS, LIFESTYLE AND MEDICAL DATA}

Information on demographics (age, gender, education), height, weight and lifestyle (smoking, use of non-steroidal anti-inflammatory drugs) was obtained at diagnosis and six months after diagnosis using self-administered questionnaires. Physical activity was assessed using the Short QUestionnaire to ASsess Health-enhancing physical activity (SQUASH)[35].

Clinical data, such as stage of disease, tumour location (colon/rectum), tumour differentiation, histological type, date of start treatment, type of treatment (surgery, neoadjuvant/adjuvant chemotherapy, radiotherapy) and presence of comorbidities (diabetes, endocrine disorders, cardiovascular, infectious, gastro-intestinal, muscular and joint, neurologic, pulmonary and urogenital diseases) were derived from the Dutch ColoRectal Audit (DCRA). This nationwide audit was initiated by the association of surgeons of the Netherlands to monitor, evaluate and improve CRC care [36]. 


\section{DATA ANALYSES}

Patient characteristics at diagnosis were described as numbers with percentages or medians with interquartile range (IQR) for the total study population and stratified by tertiles of the ADII. In addition, patients characteristics for patients with and without data available about inflammation markers were compared using descriptive statistics.

The association between the ADII (continuous) and the inflammatory markers (IL6, IL8, IL10, TNF $\alpha$, and hsCRP) was assessed cross-sectionally at diagnosis and six months after diagnosis using quantile regression analyses [37]. Based on literature the following covariates were added to the model: age, sex, stage of disease, smoking status, BMI, use of NSAIDs and having comorbidities [38-41]. Education level, physical activity, the use of statins, use of supplements and cancer type (colon vs rectal), did not influence the effect estimate, at either one of the two time points, by more than $10 \%$ and were thus not taken into account in the models. In a sensitivity analyses, we excluded patients with an acute infection (CRP > $10 \mu \mathrm{g} / \mathrm{ml}$ ).

Restricted cubic splines (RCS) analyses were used to explore and visualize the association between the ADII and recurrence and all-cause mortality. The SAS Macro \%RCS_Reg vs1.44 was used. Since linear associations were observed, the association between the ADII (continuous) and CRC recurrence and all-cause mortality was assessed using Cox proportional hazard models.

The following covariates were added to the Cox proportional hazard model based on literature: age, sex and stage of disease $[24,26]$. Smoking status, education level, BMI, physical activity, the use of NSAIDs and statins, use of supplements, tumour location (colon vs rectal) and comorbidities, did not influence the effect estimate by more than $10 \%$ at either one of the two time points and were thus not taken into account in the models. Statistical analyses were performed in SAS 9.4 (SAS Institute, Cary NC). P-values < 0.05 were considered statistically significant.

\section{RESULTS}

Of the included 1,478 CRC patients (Figure 1) 534 (36\%) were female (Table 1). The median age was 66 [IQR 61-72] year. Two-third of the patients had colon cancer. The majority of patients had no distant metastases at presentation with $24 \%$ stage I, $27 \%$ stage II, $41 \%$ stage III and $8 \%$ stage IV of disease. Patients with a more pro-inflammatory diet (tertile 3 ) compared with a more anti-inflammatory diet (tertile 1 ) were more often male, had more often a lower education level, were more often current smokers, were less physical active, and used less often supplements and statins. The inflammatory potential of the diet was rather stable between diagnosis and six months after diagnosis in the study population, as for only $15 \%$ the ADII score changed with more than 1 standard 
deviation (2.7 points). Median change for the total population was 0.1 points (IQR -1.3; 1.2). During a median follow-up of 3.2 years [IQR 2.0-4.1] for recurrence and a median follow-up of 4.8 years [IQR 3.6-5.9] for all-cause mortality, 228 recurrences and 279 deaths occurred. For the post-diagnostic measurements the median follow-up was 2.8 years [IQR 1.7-3.8] for recurrence and 4.6 years [IQR 3.4-5.6] for all-cause mortality, in which 184 recurrences and 210 deaths occurred.

Table 1 Baseline characteristics of CRC patients, stratified for tertiles of the adapted dietary inflammatory index.

\begin{tabular}{|c|c|c|c|c|}
\hline & \multirow{4}{*}{$\begin{array}{l}\text { Total population } \\
(n=1478)\end{array}$} & \multicolumn{3}{|c|}{ ADII Tertiles } \\
\hline & & Tertile 1 & Tertile 2 & Tertile 3 \\
\hline & & $(-12.2$ to $<-1.0)$ & $(-1.0$ to $<1.2)$ & $(1.2$ to $<8.5)$ \\
\hline & & $N=492$ & $N=493$ & $N=493$ \\
\hline Age (years) & 66.2 [61.3-71.6] & 66.4 [61.3-70.9] & 66.1 [61.6-71.6] & $66.2[60.8-72.4]$ \\
\hline Gender, female & $534(36)$ & $226(46)$ & $169(34)$ & $139(28)$ \\
\hline BMI $\left(\mathrm{kg} / \mathrm{m}^{2}\right)$ & $26.0[24.0-28.7]$ & $25.7[23.8-28.5]$ & $26.4[24.1-29.0]$ & $26.0[24.1-28.7]$ \\
\hline Unknown & 27 & 7 & 9 & 11 \\
\hline \multicolumn{5}{|l|}{ Education $^{a}$} \\
\hline Low & $615(42)$ & $190(39)$ & $202(42)$ & $223(46)$ \\
\hline Medium & $378(26)$ & $118(24)$ & $126(26)$ & $134(28)$ \\
\hline High & $455(31)$ & $176(36)$ & $156(32)$ & $123(26)$ \\
\hline Unknown & 30 & 8 & 9 & 13 \\
\hline \multicolumn{5}{|l|}{ Smoking habits } \\
\hline Current & $159(11)$ & $26(5)$ & $49(10)$ & $84(17)$ \\
\hline Former & 855 (59) & $290(60)$ & $293(61)$ & $272(57)$ \\
\hline Never & $436(30)$ & $169(35)$ & $142(29)$ & $125(26)$ \\
\hline Unknown & 28 & 7 & 9 & 12 \\
\hline $\begin{array}{l}\text { Physical activity }{ }^{\text {b }} \\
\text { (hours/week) }\end{array}$ & $10.5[5.0-19.0]$ & $12.0[6.0-19.0]$ & $10.0[5.0-19.0]$ & $8.5[3.6-18.2]$ \\
\hline Unknown & 2 & 0 & 1 & 1 \\
\hline Supplement use (yes) & $646(44)$ & $263(53)$ & $222(45)$ & $161(33)$ \\
\hline Unknown & 2 & 0 & 1 & 1 \\
\hline \multirow[t]{2}{*}{ Use of NSAIDs (yes) } & $183(12)$ & $60(12)$ & $53(11)$ & $70(14)$ \\
\hline & 28 & 7 & 9 & 12 \\
\hline Use of Statins (yes) & $291(20)$ & $117(24)$ & $97(20)$ & $77(16)$ \\
\hline \multicolumn{5}{|l|}{ Type of cancer } \\
\hline Colon & 995 (67) & $347(71)$ & $322(65)$ & $326(66)$ \\
\hline Rectum & $483(33)$ & $145(29)$ & $171(35)$ & $167(34)$ \\
\hline \multicolumn{5}{|l|}{ Tumour stage } \\
\hline I & $351(24)$ & $130(26)$ & $100(20)$ & $121(25)$ \\
\hline II & $401(27)$ & $132(27)$ & $141(29)$ & $128(26)$ \\
\hline III & $610(41)$ & $198(40)$ & $211(43)$ & $201(41)$ \\
\hline IV & $112(8)$ & $31(6)$ & $39(8)$ & $42(9)$ \\
\hline Unspecified & $4(0.3)$ & $1(0.2)$ & $2(0.4)$ & $1(0.2)$ \\
\hline
\end{tabular}




\begin{tabular}{lllll} 
Comorbidities & & & \\
Yes & $978(67)$ & $328(67)$ & $336(68)$ & $314(65)$ \\
Unknown & 14 & 5 & 2 & 7 \\
\hline
\end{tabular}

BMI: Body Mass Index; NSAIDs; Non-Steroidal-Anti-Inflammatory Drugs

Values presented are median [quartile 1 - quartile 3] or number (percentage).

a: Low education was defined as primary school and lower general secondary education; medium as lower vocational training and higher general secondary education; high as high vocational training and university. b: Activities with a Metabolic Equivalent score (MET score) $\geq 3$ were defined as moderate to vigorous physical activity

\section{THE INFLAMMATORY POTENTIAL OF THE DIET AND PLASMA INFLAMMATION MARKERS}

Higher ADII scores at diagnosis as well as six months after diagnosis were statistically significantly associated with higher levels of TNF $\alpha(\beta(95 \% \mathrm{Cl}) 0.03(0.01 ; 0.05) \& 0.04$ $(0.00 ; 0.07)$, respectively) and borderline significant for the summary z-score $(\beta(95 \% \mathrm{Cl})$ $0.01(-0.0 ; 0.02) \& 0.01(-0.00 ; 0.02)$, respectively) (Table 2$)$. Six months after diagnosis higher ADIl scores were associated with higher levels of hsCRP $(\beta 0.0995 \% \mathrm{Cl} 0.03 ; 0.14)$ and IL8 ( $\beta 0.0995 \% \mathrm{Cl}-0.00 ; 0.18)$. No statistically significant associations between the ADII score and IL6 and IL10 were observed (Table 2). Similar results were observed when excluding patients with an acute infection (CRP $>10 \mu \mathrm{g} / \mathrm{ml}$ ) (data not shown).

Table 2 Results of quantile regression analysis for the association between the ADII scores and the inflammatory markers

\begin{tabular}{|c|c|c|c|c|}
\hline & \multicolumn{2}{|r|}{ Crude } & \multicolumn{2}{|r|}{ Adjusted* } \\
\hline & $\mathbf{n}$ & $\beta$-coefficient 95\%Cl & $\mathbf{n}$ & $\beta$-coefficient $95 \% \mathrm{Cl}$ \\
\hline \multicolumn{5}{|l|}{ At diagnosis } \\
\hline hs-CRP & 1033 & $0.11(0.01 ; 0.21)$ & 1025 & $0.05(-0.04 ; 0.14)$ \\
\hline IL-6 & 522 & $0.02(-0.00 ; 0.05)$ & 519 & $0.01(-0.02 ; 0.03)$ \\
\hline IL-8 & 524 & $-0.03(-0.17 ; 0.12)$ & 521 & $0.01(-0.10 ; 0.13)$ \\
\hline IL-10 & 505 & $-0.00(-0.01 ; 0.00)$ & 502 & $-0.00(-0.01 ; 0.00)$ \\
\hline TNF- $\alpha$ & 512 & $0.03(0.01 ; 0.05)$ & 509 & $0.03(0.01 ; 0.05)$ \\
\hline Inflammatory z-score & 520 & $0.01(0.00 ; 0.02)$ & 517 & $0.01(-0.00 ; 0.02)$ \\
\hline \multicolumn{5}{|c|}{ Six months after diagnosis } \\
\hline hs-CRP & 1058 & $0.12(0.08 ; 0.16)$ & 996 & $0.09(0.03 ; 0.14)$ \\
\hline IL-6 & 448 & $0.03(0.00 ; 0.06)$ & 436 & $0.02(-0.01 ; 0.05)$ \\
\hline IL-8 & 448 & $0.12(0.06 ; 0.19)$ & 436 & $0.09(0.00 ; 0.18)$ \\
\hline IL-10 & 420 & $-0.00(-0.01 ; 0.01)$ & 410 & $-0.01(-0.01 ; 0.00)$ \\
\hline TNF- $\alpha$ & 450 & $0.03(0.00 ; 0.07)$ & 438 & $0.04(0.00 ; 0.07)$ \\
\hline Inflammatory z-score & 445 & $0.02(0.01 ; 0.03)$ & 433 & $0.01(-0.00 ; 0.02)$ \\
\hline
\end{tabular}

$\mathrm{Cl}$ : Confidence Interval; Hs-CRP: high sensitive C-reactive protein; IL: Interleukin; TNF: Tumour Necrosis Factor;

*Adjusted for age, sex, stage of disease, BMI, smoking status, use of NSAIDs and comorbidities.

Median (Q1-Q3) of the inflammation markers were as follows: hsCRP (mg/L), 2.4 (1.1-5.9); IL6 (pg/ml), 1.0 (0.71.6); IL8 (pg/ml), 6.0 (4.1-8.3); IL10 (pg/ml), 0.2 (0.2-0.3); TNF $(\mathrm{pg} / \mathrm{ml}), 1.9$ (1.5-2.5) and inflammatory z-score $0.06(-0.4 ; 0.3)$ at diagnosis. hsCRP (mg/L), 1.7 (0.9-3.8); IL6 (pg/ml), 1.0 (0.7-1.7); IL8 (pg/ml), 5.0 (3.9-6.6); IL10 
$(\mathrm{pg} / \mathrm{ml}), 0.3(0.2-0.5) ; \mathrm{TNF} \alpha(\mathrm{pg} / \mathrm{ml}), 2.3(1.9-2.8)$ and Inflammatory z-score $-0.02(-0.41 ; 0.35)$ six months after diagnosis

\section{THE INFLAMMATORY POTENTIAL OF THE DIET AND CRC RECURRENCE AND ALL-CAUSE MORTALITY}

Results of RCS analyses showed no associations between the ADII at diagnosis as well as six months after diagnosis and recurrence and all-cause mortality (Figure 2).

Figure 2 Visualization of the relationship between the adapted dietary inflammatory index score and CRC recurrence and all-cause mortality using restricted cubic splines analyses.

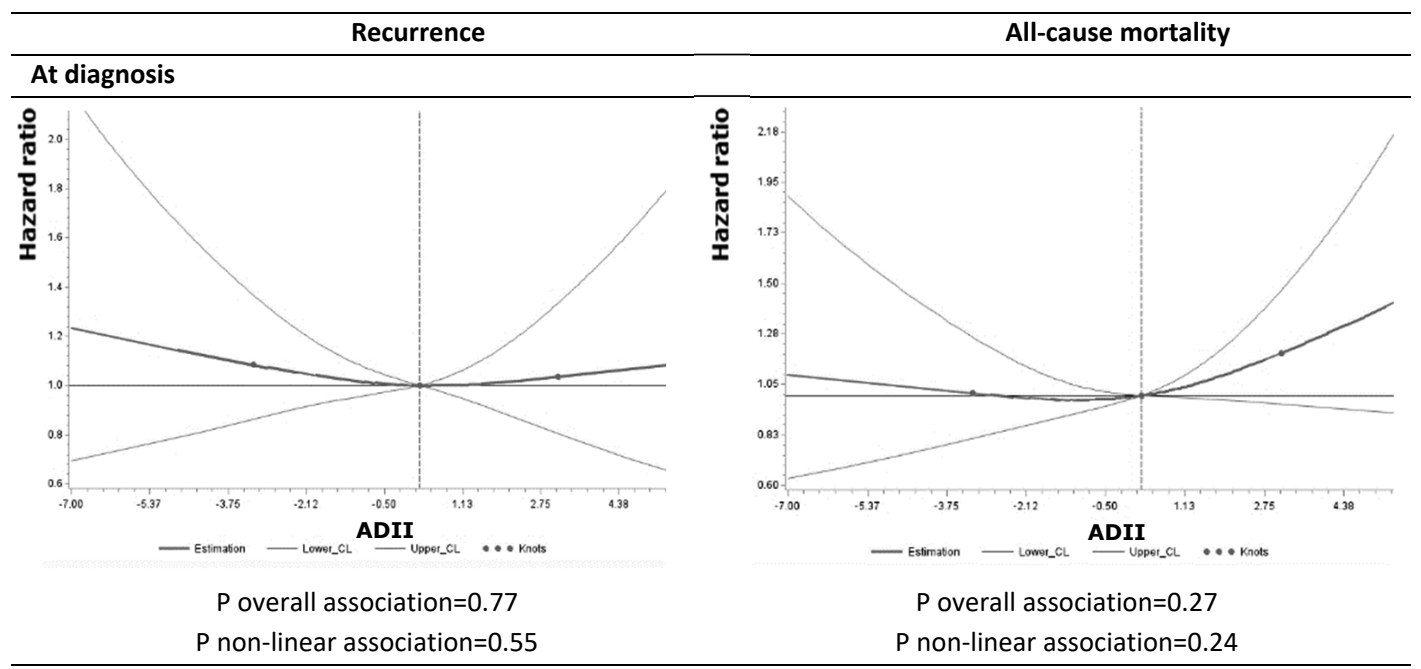

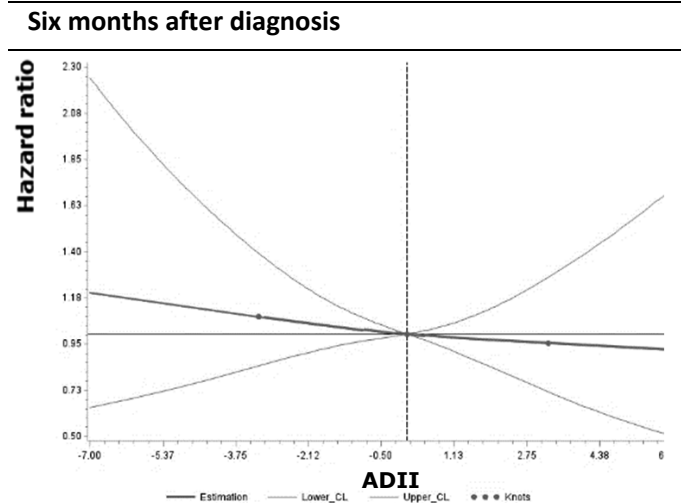

P overall association $=0.76$

$P$ non-linear association $=0.87$

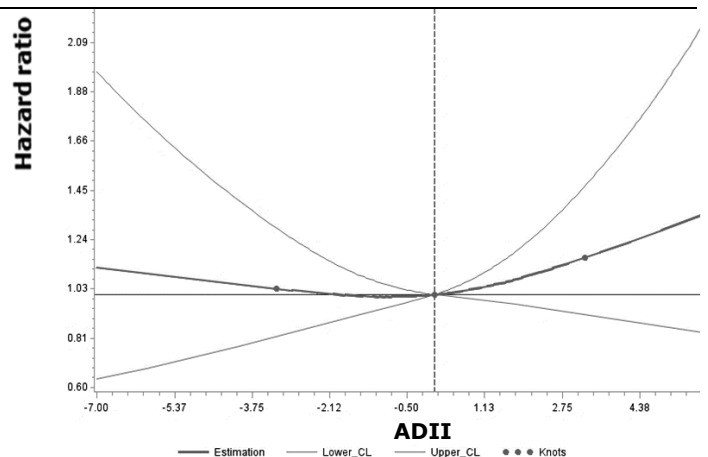

P overall association $=0.47$

P non-linear association $=0.32$

Models were adjusted for age, sex and stage of disease. Knots were placed on the $10^{\text {th }}, 50^{\text {th }}$ and $90^{\text {th }}$ percentile, where the $50^{\text {th }}$ percentile was used as the reference. 
No associations were observed between the ADII assessed at diagnosis and six months after diagnosis and recurrence and all-cause mortality (Table 3 ).

Table 3 Hazard ratios and 95\% Cl for the association between the adapted dietary inflammatory index and recurrence and all-cause mortality in CRC patients

\begin{tabular}{lcc}
\hline Risk for CRC recurrence $^{1}$ & & \\
\hline & ADIl at diagnosis & ADII six months after diagnosis \\
\hline No./Events & $1394 / 228$ & $1242 / 184$ \\
No. of cases / 1000 person years & 52 & 52 \\
Crude $\mathrm{HR}(95 \% \mathrm{Cl})$ & & $0.99(0.94-1.05)$ \\
Adjusted $\mathrm{HR}^{2}(95 \% \mathrm{Cl})$ & $1.00(0.95-1.05)$ & $0.96(0.91-1.02)$ \\
& $0.98(0.94-1.04)$ & $1335 / 210$ \\
\hline Risk for all-cause mortality & & 35 \\
\hline No./Events & $1478 / 279$ & \\
No. of deaths / 1000 person years & 39 & $1.02(0.97-1.08)$ \\
Crude $\mathrm{HR}(95 \% \mathrm{Cl})$ & & $1.00(0.95-1.05)$ \\
Adjusted $\mathrm{HR}^{2}(95 \% \mathrm{Cl})$ & $1.05(1.00-1.10)$ & \\
\end{tabular}

HR: Hazard Ratio; Cl: Confidence Interval

${ }^{1}$ Recurrence includes loco regional recurrence and/or distant metastasis. For 84 patients, data about recurrence was missing therefore these patients were excluded from the recurrence analyses.

${ }^{2}$ Adjusted for age, sex and stage of disease.

\section{DISCUSSION}

The aim of this study was to investigate the association between the inflammatory potential of the diet, measured by the ADII, and inflammatory markers, as well as recurrence and all-cause mortality in CRC patients. Higher ADII scores were associated with higher levels of TNF $\alpha$, CRP and a higher summary inflammatory z-score. However, no associations between the ADII and recurrence and all-cause mortality were observed.

In our study the ADII was associated with TNF $\alpha$, IL8 and hSCRP levels and the summary inflammatory z-score, while no associations were observed between the ADII score and IL6 and IL10. A possible reason for the null-findings for IL6 and IL10 could be the relatively low plasma levels of cytokines and the limited variation of plasma levels within the study population. The study of Woudenbergh et al., did observe a statistically significant association between the ADII score and IL6 and borderline significant associations for hsCRP and TNF $\alpha$ [20]. In that study, inflammation markers were assessed in a larger study population $(n=1,024)$ and larger variations in cytokine levels were observed, for example median and IQR levels for IL6 were $1.4 \mathrm{pg} / \mathrm{ml}$ (1.1-2.9) compared to $1.0 \mathrm{pg} / \mathrm{ml}(0.7-1.6)$ in our study [20]. Although we did not observe an association between each inflammation marker measured and the ADII score, we are confident that the ADII is reflecting the 
inflammatory potential of the diet, since it was associated with several inflammation markers in a previous study [20] and our study.

In our study no associations between the ADII at diagnosis as well as six months after diagnosis and CRC recurrence were observed when using the ADII as a continuous variable in the models. To our best knowledge, this is the first study investigating the inflammatory potential of the diet in relation to $\mathrm{CRC}$ recurrence. One reason for the lack of an association between the ADII scores and recurrence could be the limited power since we had a short follow-up period ( 2.8 years) and relatively few events $(n=184)$. Observational studies with a longer follow-up time and more events are needed to unravel the association between the inflammatory potential of the diet and CRC recurrence.

We observed no statistically significant association between a higher risk of all-cause mortality with a more pro-inflammatory diet. Results from two previous studies investigating the association between the DII and all-cause mortality in CRC patients [24, 25 ] showed either a higher risk with a more pro-inflammatory diet ( $\mathrm{HR}_{\text {tertile3vs } 1} 1.39,95 \% \mathrm{Cl}$ $1.13,1.72)[25]$ or a lower risk with a more anti-inflammatory diet at diagnosis ( $\mathrm{HR}_{\text {low v }}$ high $0.61,95 \% \mathrm{Cl} 0.38-0.99$ [24]). One previous study did observe an association between post-diagnostic and all-cause mortality ( $\mathrm{HR}_{\text {tertile1vs }}=0.49 ; 95 \% \mathrm{Cl} 0.31-0.79$ [27]). While another study observed a non-statistically significant association ( $\mathrm{HR}_{\text {continuous }}$ : $1.08 ; 95 \% \mathrm{Cl}$ : 0.97-1.20[26]). One reason for the discrepancy in the findings of our study and previous studies could be the difference in follow-up time. In those previous studies, the mean follow-up time was longer (ranging from 5 to 12 years), while our follow-up was 4.8 years for the analyses at diagnosis and 4.6 years for the post-diagnosis analyses. Another difference with previous studies is the DII score used. In most previous studies the DII [17] was used, while we used the ADII [20]. However, when repeating our analyses using the DII, we observed similar results, i.e. no associations with CRC outcomes (Table S3). Thus the different methods used to assess the inflammatory potential of the diet was not an explanation for the discrepancy in findings.

\section{FURTHER RESEARCH}

More research is needed on how to accurately assess the inflammatory potential of the diet. Investigating the influence of the inflammatory potential of the diet on CRC recurrence and survival using a nutrient-based approach provides us valuable information about the direction (protective or harmful) and strength of the inflammatory potential of the diet as a possible risk factor for recurrence and survival in CRC patients. However, the use of an index based on nutrients makes it difficult to translate results to dietary guidelines for cancer patients. Indices based on whole food groups might be more useful for translation to daily life. Therefore, it would also be valuable to investigate the influence of the inflammatory potential of the diet on CRC recurrence and survival using a 
method based on food groups. In addition, further research should also take into account molecular tumour subtype, since CRC is a heterogeneous disease and the effect of the inflammatory potential of the diet on cancer recurrence (and survival) is more likely to present in tumour with an inflammatory signature $[42,43]$.

\section{STRENGTHS AND LIMITATIONS}

Our study has some important strengths. First of all, to the best of our knowledge this is the first study investigating the association between the inflammatory potential of the diet and CRC recurrence. Second, due to the availability of detailed data on diet and other clinical and lifestyle factors, we could adjust for the most plausible confounders, although residual confounding can never be fully excluded.

Our study also had some limitations. For our ADII calculations, we were only able to include 28 of 45 food components with an inflammatory weight [17]. However, the intake of the remaining 17 components (such as ginger and turmeric) was expected to be low in the Dutch population, and we thus do not expect that this will have influenced our results. The ADII calculated with 28 food components still reflects the inflammatory potential of the diet, as higher ADII scores were associated with higher levels of IL6, sICAM and a summary inflammatory z-score for low grade inflammation in a previous study [20] and with higher levels of CRP, IL8, TNF $\alpha$ and a summary inflammatory z-score in our study. In addition, although not markedly different from the total study population, the subpopulation in which we measured cytokine levels was small $(n=524)$ and the variations in cytokine levels was rather low e.g. $1.0 \mathrm{pg} / \mathrm{ml}(0.7-1.6)$ for IL6, which limits the power to observe associations. Finally, we could not assess changes in the inflammatory potential of the diet and CRC outcomes. Because, of the 1334 patients with dietary intake data available at both time points, only 206 (15\%) showed changes of the ADII score with more than 1 standard deviation. Also, results of previous research in our groups showed that patients only marginally changed their diets after cancer diagnosis [44]. Investigating, in an intervention study, whether actively changing the inflammatory potential of the diet can improve cancer outcomes such as recurrence and mortality would be a next step to ultimately develop dietary guidelines for CRC patients. 


\section{CONCLUSION}

A higher ADII score was associated with higher levels of TNF $\alpha$, CRP and IL8. No associations with recurrence and all-cause mortality in CRC patients were observed. More research in large prospective cohort studies and stratification for molecular subtypes is needed to further unravel the association between the inflammatory potential of the diet and CRC outcomes, especially recurrence. Also investigating changes in the inflammatory potential of the diet and the association with CRC recurrence and mortality merits further investigation. 


\section{ADDITIONAL INFORMATION}

\section{FUNDING}

The COLON study was financially supported by Wereld Kanker Onderzoek Fonds (WKOF) \& World Cancer Research Fund International (WCRF International) as well as by funds from grant 2014/1179 as part of the World Cancer Research Fund International Regular Grant Programme; Alpe d'Huzes/Dutch Cancer Society (UW 2013-5927, UW 2015-7946); and ERA-NET on Translational Cancer Research (TRANSCAN:Dutch Cancer Society (UW20136397, UW2014-6877) and the Netherlands Organization for Health Research and Development (ZonMw, the Netherlands).

The funding sources had no role in the study design and conduct, data analysis and interpretation, or manuscript preparation.

\section{AUTHORS' CONTRIBUTION}

E Wesselink, LE Staritsky, E Kampman and FJB van Duijnhoven contributed to the design and the conceptualization of this study. E Wesselink, HJW de Wilt, DE Kok, AJMR Geijsen, E.A. Kouwenhoven, R.P. Veenstra, F. Kruyt and E.J. Spillenaar Bilgen and M van Zutphen contributed to recruitment of participants and the data collection and curation. Formal analyses were done by E Wesselink and L Staritsky. The manuscript was drafted by $\mathrm{E}$ Wesselink and FJB van Duijnhoven, and all authors critically read and revised the manuscript. All authors approved the final version of the manuscript.

\section{ACKNOWLEDGMENT}

The authors would like to thank the participants of the COLON study and the investigators at Wageningen University \& Research and the co-workers from the following hospitals for their involvement in recruitment for the COLON study: Hospital Gelderse Vallei, Ede; Radboudumc, Nijmegen; Slingeland Hospital, Doetinchem; Canisius Wilhelmina Hospital, Nijmegen; Rijnstate Hospital, Arnhem; Gelre Hospitals, Apeldoorn/Zutphen; Hospital Bernhoven, Uden; Isala, Zwolle; ZGT, Almelo; Martini Hospital, Groningen; Admiraal de Ruyter Hospital, Goes/Vlissingen. 


\section{REFERENCES}

[1] Shacter E, Weitzman SA. Chronic inflammation and cancer. ONCOLOGY-WILLISTON PARK THEN HUNTINGTON. 2002;16:217-29.

[2] Ward PA, Lentsch AB. The Acute Inflammatory Response and Its Regulation. Archives of Surgery. 1999;134:666-9.

[3] Terzić J, Grivennikov S, Karin E, Karin M. Inflammation and colon cancer. Gastroenterology. 2010;138:210114. e5.

[4] Chang PH, Pan YP, Fan CW, Tseng WK, Huang JS, Wu TH, et al. Pretreatment serum interleukin-1ß, interleukin6 , and tumor necrosis factor- $\alpha$ levels predict the progression of colorectal cancer. Cancer medicine. 2016;5:42633.

[5] Seruga B, Zhang H, Bernstein LJ, Tannock IF. Cytokines and their relationship to the symptoms and outcome of cancer. Nature Reviews Cancer. 2008;8:887.

[6] Xu J, Ye Y, Zhang H, Szmitkowski M, Mäkinen M, Li P, et al. Diagnostic and prognostic value of serum interleukin-6 in colorectal cancer. Medicine. 2016;95.

[7] Knupfer H, Preiss R. Serum interleukin-6 levels in colorectal cancer patients--a summary of published results. International journal of colorectal disease. 2010;25:135-40.

[8] Abtahi S, Davani F, Mojtahedi Z, Hosseini SV, Bananzadeh A, Ghaderi A. Dual association of serum interleukin10 levels with colorectal cancer. Journal of cancer research and therapeutics. 2017;13:252.

[9] Stanilov N, Miteva L, Dobreva Z, Stanilova S. Colorectal cancer severity and survival in correlation with tumor necrosis factor-alpha. Biotechnology \& Biotechnological Equipment. 2014;28:911-7.

[10] Xia W, Chen W, Zhang Z, Wu D, Wu P, Chen Z, et al. Prognostic value, clinicopathologic features and diagnostic accuracy of interleukin-8 in colorectal cancer: a meta-analysis. PloS one. 2015;10:e0123484.

[11] Woo HD, Kim K, Kim J. Association between preoperative C-reactive protein level and colorectal cancer survival: a meta-analysis. Cancer Causes \& Control. 2015;26:1661-70.

[12] Matsubara D, Arita T, Nakanishi M, Kuriu Y, Murayama Y, Kudou M, et al. The impact of postoperative inflammation on recurrence in patients with colorectal cancer. International Journal of Clinical Oncology. 2020;25:602-13.

[13] Wiseman MJ. Nutrition and cancer: prevention and survival. British Journal of Nutrition. 2018:1-7.

[14] Schlesinger S, Lieb W, Koch M, Fedirko V, Dahm CC, Pischon T, et al. Body weight gain and risk of colorectal cancer: a systematic review and meta-analysis of observational studies. Obesity Reviews. 2015;16:607-19.

[15] Arnold M, Pandeya N, Byrnes G, Renehan AG, Stevens GA, Ezzati M, et al. Global burden of cancer attributable to high body-mass index in 2012: a population-based study. The lancet oncology. 2015;16:36-46.

[16] Cavicchia PP, Steck SE, Hurley TG, Hussey JR, Ma Y, Ockene IS, et al. A new dietary inflammatory index predicts interval changes in serum high-sensitivity C-reactive protein. The Journal of nutrition. 2009;139:2365-72.

[17] Shivappa N, Steck SE, Hurley TG, Hussey JR, Hebert JR. Designing and developing a literature-derived, population-based dietary inflammatory index. Public health nutrition. 2014;17:1689-96.

[18] Sokol A, Wirth MD, Manczuk M, Shivappa N, Zatonska K, Hurley TG, et al. Association between the dietary inflammatory index, waist-to-hip ratio and metabolic syndrome. Nutrition research (New York, NY). 2016;36:1298-303.

[19] Harmon BE, Wirth MD, Boushey CJ, Wilkens LR, Draluck E, Shivappa N, et al. The Dietary Inflammatory Index Is Associated with Colorectal Cancer Risk in the Multiethnic Cohort. The Journal of nutrition. 2017;147:430-8. [20] van Woudenbergh GJ, Theofylaktopoulou D, Kuijsten A, Ferreira I, van Greevenbroek MM, van der Kallen CJ, et al. Adapted dietary inflammatory index and its association with a summary score for low-grade inflammation and markers of glucose metabolism: the Cohort study on Diabetes and Atherosclerosis Maastricht (CODAM) and the Hoorn study. The American journal of clinical nutrition. 2013;98:1533-42.

[21] Shivappa N, Godos J, Hebert JR, Wirth MD, Piuri G, Speciani AF, et al. Dietary Inflammatory Index and Colorectal Cancer Risk-A Meta-Analysis. Nutrients. 2017;9. 
[22] Li D, Hao X, Li J, Wu Z, Chen S, Lin J, et al. Dose-response relation between dietary inflammatory index and human cancer risk: evidence from 44 epidemiologic studies involving 1,082,092 participants. The American journal of clinical nutrition. 2018;107:371-88.

[23] Galas A, Kulig P, Kulig J. Dietary inflammatory index as a potential determinant of a length of hospitalization among surgical patients treated for colorectal cancer. European journal of clinical nutrition. 2014;68:1168-74.

[24] Galas A, Kulig J. Low-grade dietary-related inflammation and survival after colorectal cancer surgery. Journal of cancer research and clinical oncology. 2014;140:1517-25.

[25] Deng FE, Shivappa N, Tang Y, Mann JR, Hebert JR. Association between diet-related inflammation, all-cause, all-cancer, and cardiovascular disease mortality, with special focus on prediabetics: findings from NHANES III. European journal of nutrition. 2017;56:1085-93.

[26] Ratjen I, Shivappa N, Schafmayer C, Burmeister G, Nothlings U, Hampe J, et al. Association between the dietary inflammatory index and all-cause mortality in colorectal cancer long-term survivors. International journal of cancer Journal international du cancer. 2019;144:1292-301.

[27] Zheng J, Tabung FK, Zhang J, Murphy EA, Shivappa N, Ockene JK, et al. Post-cancer diagnosis dietary inflammatory potential is associated with survival among women diagnosed with colorectal cancer in the Women's Health Initiative. European journal of nutrition. 2019.

[28] Winkels RM, Heine-Broring RC, van Zutphen M, van Harten-Gerritsen S, Kok DE, van Duijnhoven FJ, et al. The COLON study: Colorectal cancer: Longitudinal, Observational study on Nutritional and lifestyle factors that may influence colorectal tumor recurrence, survival and quality of life. BMC Cancer. 2014;14:374.

[29] de Jager W, Bourcier K, Rijkers GT, Prakken BJ, Seyfert-Margolis V. Prerequisites for cytokine measurements in clinical trials with multiplex immunoassays. BMC immunology. 2009;10:52.

[30] Dutch Nutrition Center. NEVO Nederlandse Voedingsmiddelen tabel 2011 (In Englisch: Dutch Food Composition Table).

[31] Willett WC, Howe GR, Kushi LH. Adjustment for total energy intake in epidemiologic studies. The American journal of clinical nutrition. 1997;65:1220S-8S; discussion 9S-31S.

[32] Hu FB, Rimm E, Smith-Warner SA, Feskanich D, Stampfer MJ, Ascherio A, et al. Reproducibility and validity of dietary patterns assessed with a food-frequency questionnaire. The American journal of clinical nutrition. 1999;69:243-9.

[33] Meyer K, Ueland PM. Targeted quantification of C-reactive protein and cystatin c and its variants by immuno-MALDI-MS. Analytical chemistry. 2014;86:5807-14.

[34] Verma R, Balakrishnan L, Sharma K, Khan AA, Advani J, Gowda H, et al. A network map of Interleukin-10 signaling pathway. Journal of cell communication and signaling. 2016;10:61-7.

[35] Wendel-Vos GC, Schuit AJ, Saris WH, Kromhout D. Reproducibility and relative validity of the short questionnaire to assess health-enhancing physical activity. Journal of clinical epidemiology. 2003;56:1163-9.

[36] Van Leersum N, Snijders H, Henneman D, Kolfschoten N, Gooiker G, Ten Berge M, et al. The Dutch surgical colorectal audit. European Journal of Surgical Oncology (EJSO). 2013;39:1063-70.

[37] Staffa SJ, Kohane DS, Zurakowski D. Quantile Regression and Its Applications: A Primer for Anesthesiologists. Anesthesia \& Analgesia. 2019;128:820-30.

[38] Calder PC, Ahluwalia N, Brouns F, Buetler T, Clement K, Cunningham K, et al. Dietary factors and low-grade inflammation in relation to overweight and obesity. British Journal of Nutrition. 2011;106:S1-S78.

[39] Yanbaeva DG, Dentener MA, Creutzberg EC, Wesseling G, Wouters EF. Systemic effects of smoking. Chest. 2007;131:1557-66.

[40] Alfano CM, Peng J, Andridge RR, Lindgren ME, Povoski SP, Lipari AM, et al. Inflammatory cytokines and comorbidity development in breast cancer survivors versus noncancer controls: evidence for accelerated aging? Journal of Clinical Oncology. 2017;35:149.

[41] Rasic I, Radovic S, Aksamija G. Relationship between chronic inflammation and the stage and histopathological size of colorectal carcinoma. Medical Archives. 2016;70:104.

[42] Guinney J, Dienstmann R, Wang X, de Reyniès A, Schlicker A, Soneson C, et al. The consensus molecular subtypes of colorectal cancer. Nature Medicine. 2015;21:1350-6. 
[43] Menter DG, Davis JS, Broom BM, Overman MJ, Morris J, Kopetz S. Back to the colorectal cancer consensus molecular subtype future. Current gastroenterology reports. 2019;21:5.

[44] van Zutphen M, Boshuizen HC, Kok DE, van Baar H, Geijsen AJMR, Wesselink E, et al. Colorectal cancer survivors only marginally change their overall lifestyle in the first 2 years following diagnosis. Journal of Cancer Survivorship. 2019; 13(6): 956-967.. 


\section{SUPPLEMENTARY DATA}

Table S1: Explained interindividual variance in the adapted dietary inflammatory index by dietary components included in the calculation of the adapted dietary inflammatory index ${ }^{1}$

\begin{tabular}{lll}
\hline Component & $\begin{array}{l}\text { Partial R-square (ADII } \\
\text { at diagnosis, } \mathbf{n = 1 4 7 8 )}\end{array}$ & $\begin{array}{l}\text { Partial R-square (ADII } \\
\mathbf{6} \text { months after } \\
\text { diagnosis, } \mathbf{n = 1 3 3 4 )}\end{array}$ \\
\hline Quercetin (mg/day) & 0.41 & 0.37 \\
\hline Folic acid (mcg/day) & 0.17 & 0.20 \\
\hline Trans fatty acids (g/day) & 0.11 & 0.15 \\
\hline Vitamin D (mg/day) & 0.06 & 0.06 \\
\hline Beta carotene (mcg/day) & 0.06 & 0.05 \\
\hline Carbohydrates (g/day) & 0.04 & 0.03 \\
\hline Thiamin (mg/day) & 0.04 & 0.04 \\
\hline Alcohol (g/day) & 0.03 & 0.01 \\
\hline Saturated fatty acids (g/day) & 0.02 & 0.02 \\
\hline Fibre intake (g/day) & 0.02 & 0.02 \\
\hline Vitamin C (mg/day) & 0.01 & 0.01 \\
\hline Retinol (mcg/day) & 0.01 & 0.01 \\
\hline Omega 3 fatty acids & 0.01 & 0.01 \\
\hline Tea (g/day) & - & 0.01 \\
\hline Other components & 0.01 & 0.01 \\
\hline Forward linear regression & & 5 \\
\hline
\end{tabular}

${ }^{1}$ Forward linear regression was used to calculate the partial R2. Components that explained $>1 \%$ of the interindividual variation in the final model are shown. 
Table S2 Intake from food groups stratified by tertiles of the adapted dietary inflammatory index.

\section{ADII scores at diagnosis}

Tertile 1

$(-12.1$ to $<-1.0)$

Tertile 2

Tertile 3

$\mathrm{N}=492$

$(-1.0$ to 1.2$)$

$\mathrm{N}=493$

$(1.2$ to $<8.5)$

$\mathrm{N}=493$

Food consumption

(g/day)

\begin{tabular}{|c|c|c|c|}
\hline \multicolumn{4}{|l|}{ Meat \& Fish } \\
\hline Processed meat & $21[10-38]$ & $25[12-40]$ & $25[12-40]$ \\
\hline Red meat & 35 [19-50] & 37 [21-50] & $37[21-50]$ \\
\hline Organ meat & $0[0-4]$ & $0[0-5]$ & $0[0-3]$ \\
\hline Fish & $13[8-24]$ & $11[5-16]$ & $8[2-14]$ \\
\hline Poultry & $12[7-21]$ & $10[5-17]$ & $9[4-16]$ \\
\hline \multicolumn{4}{|l|}{ Eggs and dairy } \\
\hline Eggs & 14 [7-18] & 14 [7-18] & 14 [7-18] \\
\hline Butter & $0[0-2]$ & $0[0-2]$ & $0[0-4]$ \\
\hline Margarine & $19[7-31]$ & $16[6-26]$ & $13[3-24]$ \\
\hline Low fat dairy & $172[77-280]$ & 139 [72-249] & $127[43-236]$ \\
\hline High fat dairy & $86[35-160]$ & 78 [39-153] & $80[40-162]$ \\
\hline \multicolumn{4}{|l|}{ Drinks } \\
\hline Liquor & $0[0-1]$ & $0[0-1]$ & $0[0-4]$ \\
\hline Wine & $11[0-77]$ & $5[0-58]$ & $0[0-44]$ \\
\hline Beer & $0[0-67]$ & $11[0-108]$ & $18[0-154]$ \\
\hline Tea & 348 [232-464] & $161[36-348]$ & $80[9-232]$ \\
\hline Coffee & 348 [232-580] & 464 [232-580] & 348 [232-580] \\
\hline Fruit juices & $38[5-115]$ & $43[9-107]$ & $30[0-122]$ \\
\hline High energy drinks & $0[0-9]$ & $0[0-21]$ & $5[0-92]$ \\
\hline Low energy drinks & $0[0-8]$ & $0[0-11]$ & $0[0-5]$ \\
\hline \multicolumn{4}{|l|}{ Vegetables \& Fruits } \\
\hline Curciferous vegetables & $15[9-24]$ & $10[6-17]$ & $7[4-12]$ \\
\hline Dark yellow vegetables & $18[11-28]$ & $11[4-19]$ & $6[1-13]$ \\
\hline Tomatoes & $9[4-21]$ & $5[2-13]$ & $4[0-9]$ \\
\hline Green leafy vegetables & $23[12-38]$ & $16[8-26]$ & $11[4-19]$ \\
\hline Other vegetables & 25 [14-40] & $17[9-30]$ & 9 [3-18] \\
\hline Legumes & 24 [14-38] & $20[11-30]$ & $14[6-24]$ \\
\hline Garlic and onion & $18[7-32]$ & $10[2-21]$ & $5[0-13]$ \\
\hline Potatoes & 75 [45-90] & 75 [45-90] & 75 [45-90] \\
\hline Fruit & 213 [116-289] & 118 [69-218] & 82 [26-158] \\
\hline \multicolumn{4}{|l|}{ Grains } \\
\hline Whole grain & $130[97-171]$ & 112 [83-145] & 95 [58-131] \\
\hline Refined grain & $43[19-68]$ & 45 [24-78] & 53 [27-87] \\
\hline Breakfast cereals & $0[0-53]$ & $0[0-5]$ & $0[0-0]$ \\
\hline \multicolumn{4}{|l|}{ Others } \\
\hline Fries & $5[0-12]$ & $6[0-13]$ & $10[2-19]$ \\
\hline Pizza & $0[0-13]$ & $0[0-13]$ & $0[0-13]$ \\
\hline
\end{tabular}


CHAPTER 7

\begin{tabular}{llll}
\hline Snacks & $1[0-4]$ & $1[0-4]$ & $1[0-8]$ \\
\hline Nuts & $10[4-23]$ & $7[2-16]$ & $5[1-12]$ \\
\hline Oil and vinegar & $0[0-1]$ & $0[0-0]$ & $0[0-0]$ \\
\hline Mayonnaise & $2[1-4]$ & $2[1-4]$ & $2[0-5]$ \\
\hline Soup & $36[22-89]$ & $36[22-71]$ & $36[18-71]$ \\
\hline Sweets and desserts & $31[18-48]$ & $33[20-50]$ & $34[20-61]$ \\
\hline Condiments & $12[5-23]$ & $13[5-24]$ & $15[6-30]$ \\
\hline
\end{tabular}

Values presented are Median [Q1-Q3] 
Table S3: Hazard ratios and $95 \% \mathrm{Cl}$ for the association between the dietary inflammatory index measured using different scores (i.e. ADII and DII) and recurrence and all-cause mortality in CRC patients

\begin{tabular}{lcc}
\hline \multicolumn{2}{l}{ Inflammatory potential of the diet at diagnosis } & DII \\
\hline \multicolumn{2}{c}{ ADII } & \\
\hline Risk for CRC recurrence ${ }^{1}$ & $1394 / 228$ & $1394 / 228$ \\
\hline No./Events & 52 & 52 \\
No. of cases / 1000 person years & & $1.00(0.94-1.06)$ \\
Crude HR $(95 \% \mathrm{Cl})$ & $1.00(0.95-1.05)$ & $1.01(0.95-1.07)$ \\
Adjusted $\mathrm{HR}^{2}(95 \% \mathrm{Cl})$ & $0.98(0.94-1.04)$ &
\end{tabular}

\begin{tabular}{lcc}
\hline Risk for all-cause mortality & & \\
\hline No./Events & $1478 / 279$ & $1478 / 279$ \\
No. of deaths / 1000 person & 39 & 39 \\
years & & $1.08(1.03-1.14)$ \\
Crude $\mathrm{HR}(95 \% \mathrm{Cl})$ & $1.05(1.00-1.10)$ & $1.08(1.02-1.14)$ \\
Adjusted $\mathrm{HR}^{2}(95 \% \mathrm{Cl})$ & $1.03(0.98-1.07)$ &
\end{tabular}

Inflammatory potential of the diet six months after diagnosis

\begin{tabular}{|c|c|c|}
\hline & ADII & DII \\
\hline \multicolumn{3}{|l|}{ Risk for CRC recurrence $^{1}$} \\
\hline No./Events & $1242 / 184$ & $1242 / 184$ \\
\hline No. of cases / 1000 person years & 52 & 52 \\
\hline \multicolumn{3}{|l|}{ Crude HR (95\% Cl) } \\
\hline \multirow[t]{2}{*}{ Adjusted $\mathrm{HR}^{2}(95 \% \mathrm{Cl})$} & $0.99(0.94-1.05)$ & $0.98(0.91-1.04)$ \\
\hline & $0.96(0.91-1.02)$ & $0.97(0.91-1.03)$ \\
\hline \multicolumn{3}{|l|}{ Risk for all-cause mortality } \\
\hline No./Events & $1335 / 210$ & $1335 / 210$ \\
\hline No. of deaths / 1000 person & 35 & 35 \\
\hline \multicolumn{3}{|l|}{ years } \\
\hline Crude HR $(95 \% \mathrm{Cl})$ & $1.02(0.97-1.08)$ & $1.04(0.98-1.10)$ \\
\hline Adjusted $\mathrm{HR}^{2}(95 \% \mathrm{Cl})$ & $1.00(0.95-1.05)$ & $1.02(0.96-1.08)$ \\
\hline
\end{tabular}





\section{CHAPTER 8}

\section{GENERAL DISCUSSION}



The overarching objective of this thesis was to investigate associations between vitamin $D$ concentrations, inflammation and their interplay in relation to colorectal cancer (CRC) outcomes. To answer this objective specific aims were:

1. To investigate which factors determine vitamin $D$ concentrations in CRC patients (Chapter 2)

2. To investigate the associations between vitamin $D$ concentrations, magnesium and calcium intake and their interactions in relation to CRC outcomes (Chapter 3 )

3. To investigate changes in vitamin $D$ concentrations over time in relation to $C R C$ outcomes (Chapter 4).

4. To investigate how vitamin $D$ concentrations are associated with levels of inflammation markers over time (Chapter 5).

5. To investigate the association between inflammation markers (Chapter 6 ) and the inflammatory potential of the diet (Chapter 7) and CRC outcomes.

In this discussion chapter, a summary and schematic overview of the main results of this thesis are provided first (Figure 1). Furthermore, results are compared to existing literature. Subsequently, methodological and biological considerations, clinical implications of the main findings and potential avenues for future research are discussed. 

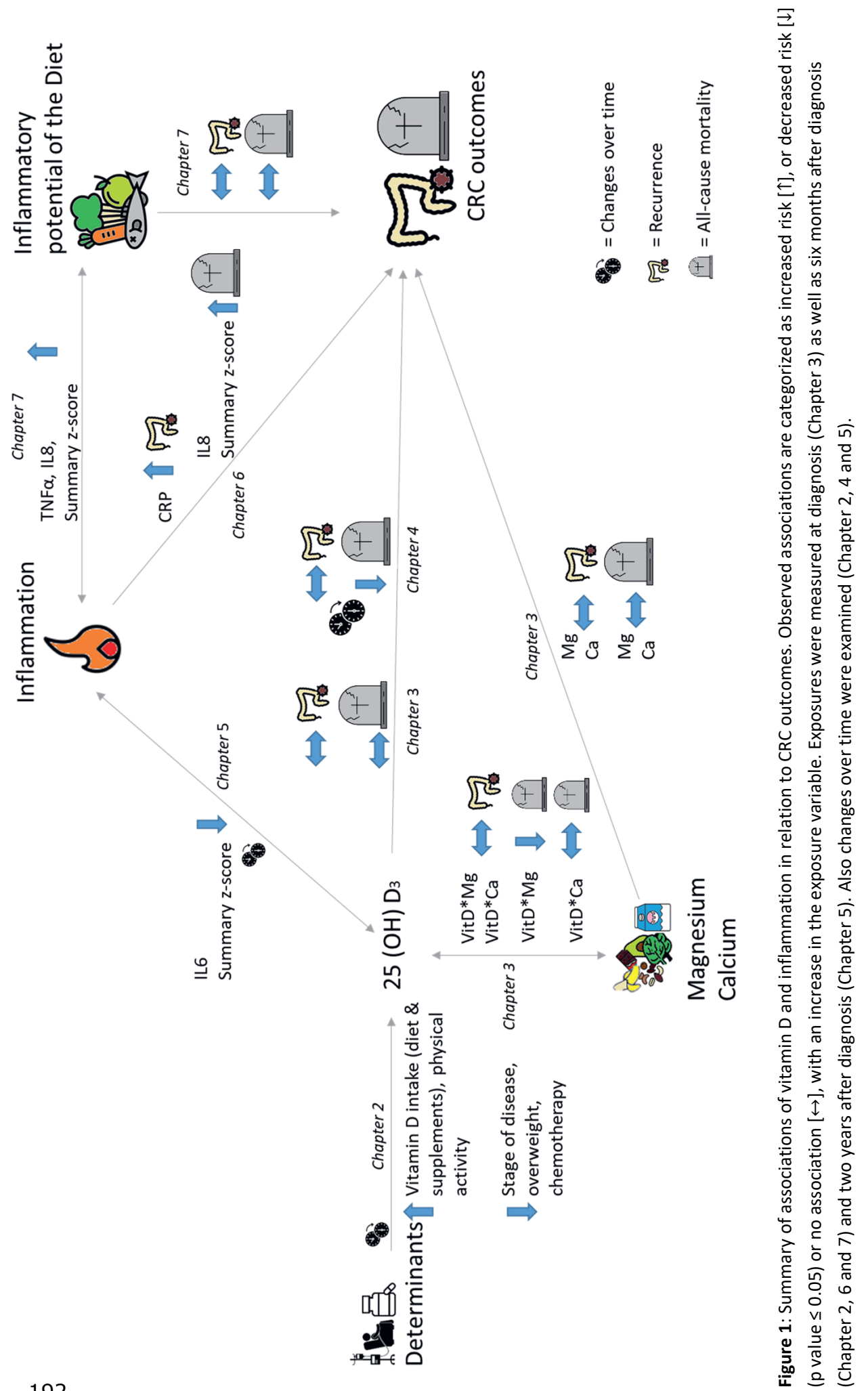


\section{INTERPRETATION OF MAIN FINDINGS}

Overall, in this thesis we observed an association between vitamin D and CRC outcomes, where higher vitamin D concentrations over time were associated with a lower risk of allcause mortality. The association between vitamin $\mathrm{D}$ and all-cause mortality was stronger in patients with a magnesium-rich diet. Higher vitamin D concentrations were associated with lower levels of inflammatory markers, while higher levels of these markers were associated with a higher risk of a recurrent event and mortality. In the following 4 statements the main findings will be discussed and compared with previous literature.

\section{StATEMENT 1: MORE ADVANCED CRC AND CHEMOTHERAPY ARE ASSOCIATED WITH LOWER SERUM 25(OH) D 3 CONCENTRATIONS}

In chapter 2, we discussed which demographic, lifestyle and clinical characteristics were associated with (changes) in $25(\mathrm{OH}) \mathrm{D}_{3}$ concentrations. Approximately half of all CRC patients were vitamin $D$ deficient ( $45 \%$ at diagnosis and $54 \%$ six months after diagnosis). This is in line with previous studies in CRC patients where prevalences ranging between 40 and $87 \%$ were observed [1-5].

We observed that several lifestyle factors were associated with $25(\mathrm{OH}) \mathrm{D}_{3}$ concentrations at diagnosis and six months after diagnosis, even after adjusting for clinical factors. This means that these lifestyle factors, such as BMI, physical activity and the use of vitamin D supplements are likely to be important determinants of vitamin D concentrations in CRC patients. Above mentioned lifestyle factors were also observed to be associated with $25(\mathrm{OH}) \mathrm{D}_{3}$ concentrations in the general population [6-10]. Next to lifestyle factors, we also investigated clinical factors in relation to $25(\mathrm{OH}) \mathrm{D}_{3}$ concentrations. We observed an association between a more advanced stage of disease and lower $25(\mathrm{OH}) \mathrm{D}_{3}$ concentrations at diagnosis. In line with our results, a previous study also observed lower concentrations of vitamin D in stage III compared to stage I and II of disease [11]. A potential explanation could be that a more advanced stage of disease and/or larger tumour size is accompanied with more inflammation $[12,13]$, which may lead to lower $25(\mathrm{OH}) \mathrm{D}_{3}$ concentrations [14]. In addition, treatment with chemotherapy was associated with lower concentrations six months after diagnosis as well as a decrease in $25(\mathrm{OH}) \mathrm{D}_{3}$ concentrations over time. In line with our findings, previous studies also observed either a higher risk of having vitamin D deficiency [15] or a decrease in $25(\mathrm{OH}) \mathrm{D}_{3}$ concentrations over time [16] in patients receiving chemotherapy. This decrease in $25(\mathrm{OH}) \mathrm{D}_{3}$ concentrations could be induced by changes in lifestyle due to chemotherapy or by chemotherapy directly. It is plausible that patients receiving chemotherapy participate less in outdoor activities, resulting in less sun exposure and subsequently lower $25(\mathrm{OH}) \mathrm{D}_{3}$ concentrations. In addition, the intestinal absorption of vitamin D could be reduced because of subclinical mucositis induced by chemotherapy [17]. Although we did not 
observe a decline over time in $25(\mathrm{OH}) \mathrm{D}_{3}$ concentrations in patients who only underwent surgery and in patients who received radiation therapy, it is likely that these treatments also affect $25(\mathrm{OH}) \mathrm{D}_{3}$ concentrations. Previous studies observed lower $25(\mathrm{OH}) \mathrm{D}_{3}$ concentrations (median ranging between 17 and $25 \mathrm{nmol} / \mathrm{L}$ ) shortly after surgery [1, 4, 18]. However, in our study follow-up samples were taken six months after diagnosis (median $25(\mathrm{OH}) \mathrm{D}_{3}$ concentrations $48 \mathrm{nmol} / \mathrm{L}$ ), which was approximately 3-4 months after surgery for most patients. As a consequence, decreased $25(\mathrm{OH}) \mathrm{D}_{3}$ concentrations may have recovered over time. To summarize, next to commonly known determinants of vitamin D status, such as vitamin D supplementation, BMI and physical activity, also cancer stage and cancer treatment influences vitamin D concentrations.

\section{StATEMENT 2: VitAMIN D IS ASSOCIATED WITH BETTER SURVIVAL, ESPECIALLY IN COMBINATION WITH MAGNESIUM}

In chapter 3 we observed a statistically non-significant higher risk of all-cause mortality in patients with severe vitamin $\mathrm{D}$ deficiency at diagnosis compared to patients with sufficient vitamin D levels (HR $1.3995 \% \mathrm{Cl} 0.87-2.21$ ). In line with our results, higher $25(\mathrm{OH}) \mathrm{D}_{3}$ concentrations were associated with a lower risk of all-cause mortality in a recent metaanalysis including 17 original studies; pooled $\mathrm{HR} 0.6495 \% \mathrm{Cl} 0.55-0.72$ [19]. Of the 17 independent cohorts included in the meta-analysis, 15 studies observed a lower risk of allcause mortality with the highest compared to the lowest quartile of $25(\mathrm{OH}) \mathrm{D}_{3}$ concentrations [19]. In the above mentioned meta-analysis of Wu et al., also a doseresponse relationship was investigated. A risk reduction of $7 \%$ for all-cause mortality was observed for each $20 \mathrm{nmol} / \mathrm{L}$ increment in 25(OH)D concentrations [19].

With respect to recurrence, we did not observe an association between $25(\mathrm{OH}) \mathrm{D}_{3}$ concentrations ( $<30 \mathrm{nmol} / \mathrm{L}$ compared to $50-75 \mathrm{nmol} / \mathrm{L}$ ) at diagnosis and recurrence, while a previous study observed a $32 \%(95 \% \mathrm{Cl} 1.03-1.71)$ higher risk of recurrence in patients with extremely low plasma $25(\mathrm{OH}) \mathrm{D}_{3}$ concentrations at diagnosis ( $\left.<11.8 \mathrm{nmol} / \mathrm{L}\right)$ compared to patients with higher $25(\mathrm{OH}) \mathrm{D}_{3}$ levels (> $45.2 \mathrm{nmol} / \mathrm{L}$ ) [4]. A reason for the discrepancy in findings could be the difference in absolute values of $25(\mathrm{OH}) \mathrm{D}_{3}$ levels and cut-off values. In the previous study $25(\mathrm{OH}) \mathrm{D}_{3}$ concentrations were lower compared to our study (median $54.5 \mathrm{nmol} / \mathrm{L}$, IQR 39.8-70.2 vs $25.2 \mathrm{nmol} / \mathrm{L}$, IQR 0.5-234.1, respectively). It could be that the associations between vitamin $D$ and recurrence are stronger, or may be only present, in patients with severe vitamin D deficiency.

In chapter 4 we observed a lower risk of all-cause mortality in all trajectories of vitamin D levels over time characterized by sufficient levels two years after diagnosis compared to a trajectory of consistent deficient levels. Sufficient vitamin D levels over time also seemed to be associated with a lower risk of recurrences compared to consistent deficient vitamin $\mathrm{D}$ levels over time. Although no other studies assessed the associations between 
(trajectories of) vitamin D concentrations over time and cancer outcomes, a previous study in which vitamin D concentrations were measured at several time points after diagnosis in different patients showed a lower risk of all-cause mortality with higher vitamin D concentrations measured before surgery as well as after surgery [18]. Thus, findings of our study and of previous studies suggest a beneficial influence of higher $25(\mathrm{OH}) \mathrm{D} 3$ concentration on cancer survival. The association with cancer recurrence is still unclear.

Furthermore, we observed that the risk of all-cause mortality was lowest in patients who had both sufficient vitamin $D$ concentrations and a high dietary magnesium intake. $A$ previous study in the general population observed a stronger inverse association between $25(\mathrm{OH}) \mathrm{D}_{3}$ concentrations and all-cause mortality in participants with a high magnesium intake compared to participants with a low magnesium intake [20]. This indicates that for an optimal health benefit both vitamin $D$ and magnesium status should be adequate. $A$ synergistic interaction between vitamin $D$ and magnesium is biologically plausible as magnesium is an essential cofactor for the enzymes involved in vitamin D activation [21]. Also, results of a randomized controlled trial that aimed to investigate the effect of magnesium supplementation on vitamin D metabolism showed that an optimal magnesium status may be important for optimizing $25(\mathrm{OH}) \mathrm{D}_{3}$ status [22]. Of note, very high concentrations of $25(\mathrm{OH}) \mathrm{D}_{3}$ due to large doses of vitamin $\mathrm{D}$ supplements can induce magnesium depletion [23]. Therefore, increasing dietary magnesium intake or magnesium supplementation should be considered in vitamin D deficient patients treated with high dose vitamin D supplements.

To conclude, vitamin D and magnesium may work synergistically in decreasing the risk of all-cause mortality in CRC patients. In addition, trajectories of vitamin D over time characterized by sufficient vitamin D concentrations ( $>50 \mathrm{nmol} / \mathrm{L}$ ) were associated with a lower risk of all-cause mortality in CRC patients. Whether $25(\mathrm{OH}) \mathrm{D}_{3}$ concentrations are also associated with $\mathrm{CRC}$ recurrence is still unclear.

\section{StATEMENT 3: A DOSE OF VITAMIN D PER DAY LOWERS INFLAMMATION, BUT DOES NOT KEEP INFLAMMATION AWAY.}

One of the suggested mechanisms by which vitamin D exerts its beneficial effects on CRC outcomes is by its anti-inflammatory properties [24]. Therefore, to reveal part of the "black box" between vitamin D and CRC outcomes, we investigated longitudinal associations between $25(\mathrm{OH}) \mathrm{D}_{3}$ concentrations and inflammation markers in chapter 5 . We observed lower levels of IL6 and the inflammatory summary z-score with higher $25(\mathrm{OH}) \mathrm{D}_{3}$ concentrations. Within individuals, higher $25(\mathrm{OH}) \mathrm{D}_{3}$ concentrations over time were additionally associated with lower IL10 and TNF $\alpha$ levels over time. However, although $25(\mathrm{OH}) \mathrm{D}_{3}$ and inflammatory cytokines were measured several times during 
follow-up, concentrations were assessed cross-sectional in the same blood sample. Therefore, it is not possible to conclude from our results whether increasing $25(\mathrm{OH}) \mathrm{D}_{3}$ decreases inflammation markers, or the other way around. Only limited research regarding vitamin $D$ and inflammation markers has been done in cancer patients $[25,26]$. Results of a recent study in CRC patients showed a weak negative correlation between $25(\mathrm{OH}) \mathrm{D} 3$ concentrations and C-reactive protein (CRP) concentrations $(r=-0.12, p=0.02$ ) [18]. Furthermore, a randomized clinical trial in colorectal adenoma patients observed a non-statistically significant decline in CRP, IL6, IL1 $\beta$ and TNF $\alpha$ concentrations after supplementation with $20 \mu \mathrm{g} /$ day of vitamin D3 for six months. Moreover, a combined inflammatory z-score decreased with $77 \%$ in the vitamin D group compared with $33 \%$ in the control group in the same RCT [27]. In patients with prostate cancer higher 25(OH)D3 concentrations were associated with lower levels of CRP ( $r=-0.38, p=<0.05)$ and IL8 ( $r=-$ $0.29, p<0.01)$ [25]. Thus, results of our and previous studies seem to suggest that $25(\mathrm{OH}) \mathrm{D}_{3}$ concentrations could decrease levels of inflammatory markers. A dose of vitamin $D$ a day may result in lower levels of inflammation, but can probably not keep inflammation away.

\section{STATEMENT 4: INFLAMMATION IS ASSOCIATED WITH WORSE CRC OUTCOMES}

In chapter 6 we showed that higher levels of several inflammation markers were associated with worse CRC outcomes. Higher CRP levels before and after surgery were associated with a higher risk of recurrence, while higher IL8 levels and the combined inflammatory z-score were associated with a higher risk of all-cause mortality. Previous studies also observed worse CRC outcomes with higher levels of inflammation markers, including IL6, IL8, TNF $\alpha$ and CRP [12, 28-34]. Taking all evidence from previous studies together we can conclude that in general, higher pre-operative as well as post-operative levels of pro-inflammatory markers are associated with worse CRC survival, whereas the association with CRC recurrence is less well investigated. Inflammation could influence cancer outcomes via several mechanisms. The most prominent one is the stimulation of NFkB and STAT3 which results in increased cell proliferation, angiogenesis, tumour growth and tumour invasion $[35,36]$. Thus, we hypothesize that reducing levels of inflammation could probably delay tumour growth and recurrence, resulting in a better overall survival. Chronic inflammation is also suggested to play a role in the onset of comorbidities such as cardiovascular diseases and diabetes [37, 38], thus associations between inflammation markers and all-cause mortality could go beyond cancer alone.

Diet is a modifiable lifestyle factor which can potentially reduce levels of inflammation, since diet has a marked effect on inflammation [39-41]. Although the adapted dietary inflammatory index (ADII) was associated with several inflammation markers, no associations between the ADII and CRC outcomes were observed in our study (Chapter 7). Apart from our study, no studies have investigated associations between the 
inflammatory potential of the diet and cancer recurrence. Results of three previous studies showed a higher risk of CRC-specific and all-cause mortality in CRC patients with a more pro-inflammatory diet (highest category) compared to an anti-inflammatory diet (lowest category) with hazard ratios ranging between 1.32 and 1.42 [42-45]. A reason for the discrepancy in findings between our study and previous studies could be the difference in follow-up time. In the previous studies, the median follow-up time was longer (ranging from 5 to 12 years), while our median follow-up was 4.8 years for the analyses at diagnosis and 4.6 years for the post-diagnosis analyses. Another difference between our study and previous studies is the method by which the inflammatory potential of the diet was assessed. The most commonly used score to assess the inflammatory potential of the diet is the dietary inflammatory index (DII) developed in 2014. Later on two updated versions were made: the adapted dietary inflammatory index (ADII), and the energy-adjusted dietary inflammatory index (EDII), which are comparable. We repeated our analyses using the DII. Similar results for the ADII and DII were observed in our study population, i.e. no associations with CRC outcomes. Thus, the difference between scores used could not explain the discrepancy between our findings and findings of previous studies. Although not observed in our study, the inflammatory potential of the diet may be associated with all-cause mortality, but further investigation is warranted. In summary, higher levels of chronic low-grade inflammation are associated with worse CRC outcomes. Whether a more anti-inflammatory diet might decrease levels of chronic lowgrade inflammation and subsequently improve CRC outcomes still needs to be elucidated.

We observed statistically significant associations between inflammation markers and $25(\mathrm{OH}) \mathrm{D}_{3}$ concentrations as well as $\mathrm{CRC}$ outcomes (Chapter 5-7). The same cytokines were, however, not consistently statistically significantly associated with the exposures (i.e. $25(\mathrm{OH}) \mathrm{D}_{3}$ concentrations and the inflammatory potential of the diet) and outcomes (i.e. recurrence and all-cause mortality). For example, IL6 was associated with $25(\mathrm{OH}) \mathrm{D}_{3}$ concentrations, IL8 and TNF $\alpha$ with the inflammatory potential of the diet, IL8 with allcause mortality and CRP with recurrence. Although this might seem random, the inflammatory z-score, which is a way to combine potentially related cytokines, showed (borderline) statistically significant associations with all investigated exposures and outcomes. Furthermore, although not statistically significant, associations for individual cytokines were in the expected direction. Thus, the combined results of this thesis implies that higher levels of pro-inflammatory markers are associated with worse CRC outcomes. 


\section{METHODOLOGICAL AND BIOLOGICAL CONSIDERATIONS}

The interpretation and validity of research findings is influenced by several methodological and biological aspects of which the most important ones will be considered below. These aspects mainly concern causality, potential confounding, the choice of inflammatory markers, colorectal cancer recurrences, data harmonization loss of follow-up, and external validity.

\section{EVIDENCE FOR A CAUSAL RELATIONSHIP BETWEEN VITAMIN D AND MORTALITY IN CRC PATIENTS}

Is vitamin D only a marker of "good health" rather than causally related to mortality? In this paragraph the evidence for a causal relationship between vitamin D and CRC outcomes will be discussed taking methodological and biological considerations into account. The Bradford Hill criteria are used as a guidance [46]. The viewpoints, temporality, consistency and strength of the association, dose-response relationship, experimental evidence, biological plausibility and specificity are discussed.

The only essential criterion for causal inference is temporality. The exposure, in our case $25(\mathrm{OH}) \mathrm{D}_{3}$ concentrations, must precede the outcome: recurrence and/or mortality. In our study and previous studies, vitamin D concentrations were assessed before the outcomes occurred. Although the $25(\mathrm{OH}) \mathrm{D}_{3}$ concentrations were measured before the event occurred, reverse causation when investigating recurrence or residual confounding when investigating survival (e.g. underlying causes of death influencing vitamin D concentrations) can still be present. One way to partly solve this problem is by excluding participants who had an event very shortly after the last exposure measurement was taken. In case of reverse causation or residual confounding related to the underlying cause of death, associations will be attenuated or even diminished after excluding patients who had a recurrent event or died shortly after the exposure was measured. Similar results regarding the association between trajectories of vitamin D over time and CRC outcomes were observed after excluding patients who died shortly after the last measurement (Chapter 4), providing evidence for a causal relationship. Thus, the criterion for temporality is met.

Three other factors when weighing evidence are the consistency and strength of an association and the presence of a dose-response relationship. In statement 2 , the main findings of our studies and previous studies regarding the associations between $25(\mathrm{OH}) \mathrm{D}_{3}$ concentrations and CRC outcomes are discussed. Briefly, results of epidemiological studies show a consistent inverse association between $25(\mathrm{OH}) \mathrm{D}_{3}$ concentrations and all-cause mortality in CRC patients. Moderate heterogeneity for all-cause mortality $\left(\mathrm{I}^{2}=52.6 \%\right)$ was observed in the meta-analysis of Wu and colleagues in 2020 [19]. This heterogeneity was caused by a study in a Chinese population with 206 stage I-III CRC patients and 74 events. 
In this particular study only 24 patients were included in the highest category of vitamin D concentrations (>75 nmol/L) and compared to 59 patients in the lowest category $(<15$ $\mathrm{nmol} / \mathrm{L})$, which could have led to unstable effect estimates as shown by the wide $\mathrm{Cl}(0.90$ 3.56). Also no correction for confounding was done [47]. Thus, no unexplained heterogeneity was observed. Moreover, a dose-response relationship was observed, a risk reduction of $7 \%$ for all-cause mortality for each $20 \mathrm{nmol} / \mathrm{L}$ increment in 25(OH)D concentrations, which increases the likelihood of a causal relationship [19]. With respect to the strength of the association, a 36\% (28-45\%) lower risk of dying was observed in the highest compared to the lowest category of $25(\mathrm{OH}) \mathrm{D}_{3}$ concentrations [19]. Even though the difference between the lowest and highest category of vitamin D concentrations can be large, this is a substantially decreased risk, which is statistically significant and clinically relevant. The stronger an association, the more likely that the associations cannot be explained by other measured or non-measured confounding factors. Within the articles of this thesis, but also in most previous studies cited, adjustment for confounding has been applied to prevent any interference with other measured variables in the studied association. However, due to the observational nature of this study residual confounding can never be excluded. Serum vitamin D levels, $25(\mathrm{OH}) \mathrm{D}_{3}$ were adjusted for week of blood collection using the locally weighted polynomial regression (LOESS) method, which is a more sophisticated and accurate way to correct for seasonal variance compared to adding season as a variable to the models $[48,49]$. Thus the association observed in epidemiological studies between vitamin D and all-cause mortality in CRC survivors is likely not entirely caused by confounding variables such as stage of disease (adjusted for in all but one of the conducted studies) or other demographic or lifestyle factors (extensively adjusted for in most conducted studies). Taken together, a moderately strong and consistent association between vitamin D and CRC and all-cause mortality was observed in epidemiological studies.

A method called mendelian randomization is often used in epidemiological studies to overcome unmeasured confounding and to judge causality. Mendelian randomization uses genetic variation to investigate the causality of a relationship [50]. However, a disadvantage of this method is the large number of participants needed $(n>15.000)$ to perform such an analysis, because genetic variants usually explain only a very small proportion of the exposure variation. Mendelian randomization studies conducted so far, did not observe associations between genetic risk scores of vitamin D concentrations and CRC outcomes [51-55]. However, most of these studies were underpowered [51, 53-55] and the genetic risk scores explained $\leq 5 \%$ of the variability in serum $25(\mathrm{OH}) \mathrm{D}_{3}$ concentrations [51-55]. Since the genetic risk scores only explained less than $5 \%$ of circulating vitamin D concentrations it is questionable whether mendelian randomization, is valuable when investigating vitamin $\mathrm{D}$ and $\mathrm{CRC}$ outcomes. 
In addition, experimental trials were conducted to investigate the effect of vitamin D supplementation on mortality. A reduced total cancer mortality rate was observed with daily vitamin D supplementation in the general population [56]. Moreover, two randomized controlled trials investigated vitamin $D$ supplements in relation to CRC prognosis in CRC patients $[57,58]$. Four other trials assessed the effect of vitamin $D$ supplementation on CRC mortality in population cohorts [59-62]. Recently (2020), a metaanalysis was conducted combining results of 5 of the intervention studies mentioned above [57-60, 62]. A pooled $\mathrm{HR}$ of $0.67(95 \% \mathrm{Cl} 0.40-0.94)$ was observed for progression free survival and a pooled $\mathrm{HR}$ of $0.76(95 \% \mathrm{Cl} 0.39-1.13)$ was observed for CRC-specific survival [26]. Although longer follow-up of minimally 5 years in these trials is needed to obtain better estimates of effects on survival, experimental evidence tentatively supports a lower risk of all-cause mortality after vitamin D supplementation, which is suggestive of a causal relationship.

Elucidation of biological pathways underlying the association between vitamin D and CRC outcomes contributes to the likelihood of a causal relationship. Results of animal and invitro studies support evidence for a potential causal relationship between vitamin $D$ and CRC outcomes, as vitamin D has several anti-cancer and anti-inflammatory effects [24]. In addition, nowadays more advanced epidemiological techniques are available to understand complex associations between exposures and disease progression. For example, a mediation analysis allows for the disentanglement and decomposition of biological pathways of direct and indirect effects that play a role in filling in the "black box" between vitamin D and cancer prognosis. Originally, we aimed to perform such a mediation analysis [63] to investigate whether inflammation is one of the underlying mechanism for the association between vitamin D and CRC prognosis. Unfortunately, we did not have enough power to investigate this, as we only had inflammation markers available in a subgroup of our population ( $n \pm 750)$. In this subgroup with a limited number of events ( $n=82$ ), no association between vitamin $D$ and CRC outcomes was observed, while we did observe an association between $25(\mathrm{OH}) \mathrm{D}_{3}$ concentrations and all-cause mortality in the overall population. Therefore, it was not possible to conduct a mediation analysis. Although not fully unraveled yet, emerging evidence suggests that a plausible biological mechanism underlying the association between vitamin D and CRC outcomes is present.

The association between $25(\mathrm{OH}) \mathrm{D}_{3}$ and $\mathrm{CRC}$ outcomes is not specific, as vitamin $\mathrm{D}$ deficiency is linked to several health outcomes of which bone health is the most well understood. Also all-cause mortality has multiple causes and risk factors. Unfortunately, we had no data available about the causes of death. Therefore, we were not able to perform analyses with disease-specific mortality as an outcome. Although we could not assess CRC-specific mortality in our study, previous studies did. These previous studies consistently observed an inverse association between $25(\mathrm{OH}) \mathrm{D}_{3}$ concentrations and CRC- 
specific mortality (pooled HR $0.6595 \% \mathrm{Cl} 0.56-0.73$ ) [19]. Non-specificity is not a reason to rule out causality. Many factors contribute to the risk of all-cause mortality and vitamin $D$ could be one of them.

Taken together, although caution is warranted in drawing conclusions about cause and effect based on analyses in this thesis, conducted in a prospective observational cohort study, results of this thesis (and previous research) give a foundation for a causal relationship. Before conclusions can be drawn about causality, 1) underlying mechanisms should be further clarified preferably in human studies and 2) randomized controlled trials investigating the effect of improving and optimizing $25(\mathrm{OH}) \mathrm{D}_{3}$ concentrations in relation to recurrence and CRC-specific mortality should be conducted. For more details see the paragraph on future research later on in this discussion.

\section{THE CHOICE FOR CIRCULATING INFLAMMATORY CYTOKINES AS MARKERS OF INFLAMMATION}

A first point to consider when investigating inflammation is which inflammatory marker should be measured to accurately capture inflammation. In large epidemiological studies it is often possible to collect blood samples. However, large-scale collection of tissue is challenging. Furthermore, blood samples are often collected and processed at multiple sites and under variable conditions. These two points should be kept in mind when deciding which inflammatory markers to measure. Several parameters of inflammation are primarily present within cells and thus not expected to be present in the circulation. Examples are NFKB and STAT3, which would be interesting to measure as they play a central role in the suggested underlying mechanism between vitamin $D$ and CRC prognosis. These transcription factors, however, can only be analyzed in immune cells and not in plasma [64]. Another example is COX2 which can only be detected in tissue. Although lipid-derived mediators such as prostaglandins, which are products of COX2, are measurable in plasma [65], they are chemically instable. This makes these lipid-derived mediators often impractical to include when samples are collected at multiple sites under variable conditions. Soluble peptides and proteins, such as cytokines and CRP are relatively stable in plasma [66]. Thus cytokines and CRP, are inflammatory markers most accessible to be measured in blood plasma collected in epidemiological studies. We chose to assess cytokines regulated by vitamin D and involved in CRC progression [64].

Another point of consideration is the storage and analyses of inflammatory markers. Blood samples were collected over an extended timespan in our study. Improper or extended storage may result in degradation of cytokines. Cytokines were shown to remain stable in plasma for a period of up to 2 years of storage at $-80^{\circ} \mathrm{C}$ [66]. Therefore, we only measured inflammatory markers in samples stored $<2$ years. However, because of this, samples of an individual collected at different time-point during follow-up could not be analyses at the same time. This resulted in inter-assay variability. To get insights in inter- 
assay variability over time, plasma of 16 healthy individuals was collected, mixed and used as an additional control next to the standard manufactory controls. We added this plasma control to all plates, during all rounds of measurements, to check for inter-plate variability and stability of samples over time. For the manufacturer's controls inter and intra-batch coefficients for all cytokines were $<8 \%$. For the heathy control plasma sample the average inter-batch coefficient for IL10 was 34\%, for IL6 27\%, for IL8 11\% and for TNF $18 \%$. The average intra-batch coefficient for IL-10 was 16\%, for IL6 8\%, for IL8 5\% and for TNF $6 \%$. Although the inter-batch variation seems to be high for IL10 and IL6, the detected differences between patients was higher than the measurement insecurity as variation in IL6 concentrations was higher in our study population (median $1.0 \mathrm{\rho g} / \mathrm{ml}$, IQR 0.7-1.7) compared to the healthy control plasma sample (median $0.3 \rho \mathrm{g} / \mathrm{ml}$, IQR $0.2-0.4 ; 27 \%$ of $0.3=0.08 \rho \mathrm{g} / \mathrm{ml})$.

In conclusion, inflammatory cytokines are the most reliable markers of inflammation in the setting of a large prospective cohort study and we are confident that the inflammatory marker data used in our analyses is of high quality.

\section{COLORECTAL CANCER RECURRENCE}

Within the literature several definitions of recurrence can be found [67]. Varying definitions for cancer recurrence hinders comparisons between studies and may introduce error in the outcome assessment [67]. In this thesis recurrence was defined as a locoregional recurrence or distant metastasis. Of the patients included in the COLON and EnCoRe studies who got a recurrent event (13\%), the majority had a distant metastasis $(88 \%)$, while only $12 \%$ had a locoregional recurrence. Thus, to study vitamin D and other lifestyle related factors in relation to recurrence in general and especially to local recurrences, large sample sizes are needed. This is also one of the reasons why the relation between vitamin $D$ and recurrence has hardly been studied so far. Another reason is that collecting data about tumour recurrences is more expensive and time-consuming compared to collecting data about vital status and cause of death.

Most recurrent events occur in the first three years after diagnosis [68], while cancer develops over many years [69]. Because of this relatively short time-span it can be argued that the recurrence escaped tumour treatment, or that it was already present at time of diagnosis in a different part of the body. This raises the question whether vitamin $D$ concentrations and lifestyle after diagnosis can still influence recurrence. Vitamin $D$ has several anti-inflammatory and anti-cancer properties [70] and inflammation plays an important role in CRC progression [35], as discussed in more detail in the introduction. We hypothesize that improving vitamin D concentrations and decreasing the inflammatorypotential of the diet could reduce inflammation and thereby slow down the growth of the recurrent tumour and hamper metastasis. 


\section{DATA HARMONIZATION ACROSS COHORT STUDIES}

To answer complex questions about (changes in) vitamin D and dietary factors in relation to CRC outcomes, collaborations with other cohorts are required to have enough power to detect relative small associations as expected in nutritional research. However, one of the challenges when combining data of two or more cohorts is data harmonization. In this thesis we used data from two prospective cohort studies in the Netherlands. To be able to conduct our analyses, demographic, clinical, nutritional, and lifestyle information as well as biomarkers needed to be harmonized across cohorts. A major strength was that vitamin $D$ concentrations as well as inflammatory marker levels were assessed in the same laboratory for both cohorts. Circulating serum $25(\mathrm{OH}) \mathrm{D}_{3}$ concentrations were assessed using a high quality and state-of-the-art technique. Liquid chromatography coupled to tandem-mass spectrometry (LC-MS/MS) is the superior and preferred method compared to the traditional and more widely used automated radioimmunoassay [71-73]. Levels of inflammatory markers were measured at our lab at Wageningen University \& Research using a multiplex assay. Also recurrence data and data about mortality was assessed using the same approach (i.e. via linkages with the Netherlands Cancer Registry and the Municipal Personal Records Database, respectively). Thus, main exposures and main outcomes were measured in the same way. The two main differences between the COLON and EnCoRe study were the time points of measurements and the dietary assessment methods. In the COLON study, measurements were done at diagnosis, and at six months, one year and two years after diagnosis, while in the EnCoRe study measurements were done at diagnosis, and at six weeks, six months, one year and two years after the end of treatment. To be able to harmonize the data of both cohorts for analyses at several time points after CRC diagnosis, we selected the time point in the EnCoRe cohort closest to either six months, one year and two years after diagnosis as the second, third and fourth time points. Also, dietary assessment methods were different between the two studies. Habitual dietary intake in the month (COLON study) or year (EnCoRe study) preceding diagnosis was assessed using a semi-quantitative food frequency questionnaire (FFQ). At the other time points, habitual dietary intake was assessed with a FFQ in the COLON study and a 7-day food diary in the EnCoRe study. Although not similar, the FFQs from COLON and EnCoRe were comparable. Both FFQs captured all food groups that are considered main contributors to dietary vitamin D (e.g. fatty fish, eggs), dietary magnesium (e.g. whole grain products, nuts and vegetables) and dietary calcium intake (e.g. dairy products). Furthermore, for both FFQs the amount of vitamin D, magnesium or calcium was calculated in the same way using the same food composition table. In addition, we made cohort-specific quartiles. This is because, although absolute values may differ due to differences in the assessment method, we were still able to rank participants based on their intake of vitamin D, magnesium and calcium. Finally, dietary intake was only used as the exposure of interest in one research question (Chapter 3 ). In all other analyses, dietary 
intake was taken into account as a potential confounder. In summary, biomarkers $\left(25(\mathrm{OH}) \mathrm{D}_{3}\right.$ and inflammatory markers), which were the main exposures of interest, and recurrence and mortality, which were the main outcomes of interest, were measured in exactly the same way in both studies. Differences in timing of follow-up measurements and dietary assessment methods are not likely to have influenced observed associations. Thus data harmonization led to a larger sample size without reducing accuracy of data assessment, which resulted in more statistical power.

\section{EXTERNAL VALIDITY}

Before providing some ideas about the clinical implications of this study, we should be aware of an important question namely: are participants of the COLON and EnCoRe study, in particular those who donated blood at several timepoints, representative for the CRC patients seen in daily clinical practice? If so, findings of this thesis can be generalized to the general population of CRC patients/survivors. In most analyses regarding CRC outcomes stage I-III CRC patients were included (Chapter 2-4 and 6). Stage IV patients were included in the analyses regarding the association between vitamin $D$ and inflammation markers (Chapter 5 ) and the association between the ADII and CRC outcomes (COLON study only; Chapter 7). Thus, findings of this thesis can be generalized to stage I-III CRC patients, but not to stage IV CRC patients.

For the analyses regarding vitamin D and inflammation markers (Chapter 2-6), only patients who donated blood were included. At baseline, characteristics of the patients who donated blood were not different from patient who did not donate blood. However, at follow-up time points especially the two year follow-up timepoint (Chapter 4 and 5), patients who donated blood seemed to be slightly healthier and had a better prognosis compared to the total CRC population in the Netherlands [74]. This could potentially hamper external validity, especially for the findings regarding changes over time (Chapter 2, 4 and 5). On the other hand, when repeating the analyses regarding baseline vitamin D concentrations and CRC outcomes within the specific study population with blood samples available at all follow-up timepoints, slightly stronger yet not statistically significant associations were observed. The comparable results indicate that our population for analyses regarding changes in vitamin D concentrations is not a selected population, however, they seemed to have a greater chance of survival compared to CRC patients in general [74]. Thus, the results regarding trajectories of vitamin D over time (Chapter 4) could only be generalized to stage I-III CRC patients with a relatively good prognosis. 


\section{CLINICAL IMPLICATIONS}

Based on the results of this thesis and current literature available, two preliminary guidelines are made: one regarding vitamin $\mathrm{D}$ supplementation and one about chronic inflammation.

\section{Preliminary guideline 1: Vitamin D SUPPlementation in COMBinAtion With MAgNeSiUm AND CALCIUM SHOULD BE ADVISED TO CRC PATIENTS WITH INSUFFICIENT VITAMIN D CONCENTRATIONS.}

Approximately half of all CRC patients are vitamin D deficient (Chapter 2). We observed that patients who had a deficient $25(\mathrm{OH}) \mathrm{D}_{3}$ status appeared to be more often male, obese, had a lower level of physical activity, a more advanced disease stage, and were less often using vitamin $\mathrm{D}$ and calcium supplements compared with patients who had sufficient vitamin D concentrations (Chapter 2). In addition, chemotherapy was associated with a decline in vitamin D concentrations. Given the high prevalence of vitamin $D$ deficiency in CRC patients, vitamin D concentration should be monitored, especially in patients with one or more of the abovementioned characteristics. Temporarily low $25(\mathrm{OH}) \mathrm{D}_{3}$ concentrations seem not to be harmful as long as levels restore afterwards (Chapter 4). In addition, better survival rates were observed in patients with sufficient vitamin D concentrations two years after diagnosis. Therefore, increasing vitamin D concentrations could be beneficial for CRC patients. If vitamin D concentrations are still low ( $<50 \mathrm{nmol} / \mathrm{L}$ ) one year after CRC diagnosis, vitamin D supplementation is recommended, as availability of vitamin $D$ is limited in our diet and synthesis of vitamin $D$ in the skin decreases with age [10]. Since magnesium and calcium are closely linked to vitamin D metabolism, adequate intake/concentrations of all three nutrients is necessary (Chapter 3). Preferable by means of a vitamin D supplement combined with a diet rich in magnesium and sufficient in calcium. If it is not possible to increase magnesium and calcium via dietary intake, a combined supplement of vitamin $D$, magnesium and calcium is recommended. The Dutch Health Council recommends an additional intake of vitamin D by means of supplementation of $10 \mu \mathrm{g} /$ day for women between 50 and 69 years of age and of $20 \mu \mathrm{g} /$ day for all individuals above the age of 70 in the general population $[75,76]$. In our study population, only $23 \%$ of the male participants $\geq 70$ years and $34 \%$ of the female participants $\geq 50$ years followed this recommendation. Stimulating cancer survivors to stick to the guidelines regarding vitamin $D$ for the general population can probably already improve vitamin D status. In case of severe deficiencies ( $<30 \mathrm{nmol} / \mathrm{L}$ ) higher starting dosages as a boost are needed [77]. Around $70 \%$ of Dutch geriatricians and general practitioners are aware of the vitamin $D$ supplementation recommendations of the Dutch Health Council and about half of them prescribed vitamin D for older adults $>70$ years [78]. Increasing the awareness of the high prevalence of vitamin D deficiencies in CRC patients among health care professionals would contribute to a more active approach toward reducing the prevalence of vitamin $D$ deficiencies. It can be presumed that 
dosages up to $100 \mu \mathrm{g} /$ day can be safely administered [77]. Of note, clinicians should be aware that vitamin $\mathrm{D}$ supplementation interacts with some commonly prescribed drugs in older adults, such as statins (could reduce vitamin D synthesis) and lipase inhibitors (reduce vitamin D absorption) [79]. In case of vitamin D deficiency, vitamin D supplementation should be taken several hours before the dose of medication to minimize interaction.

A continued debate is going on with respect to the optimum cut-off value for deficient serum $25(\mathrm{OH}) \mathrm{D}_{3}$ concentrations. Vitamin $\mathrm{D}$ deficiency is generally defined as circulating $25(\mathrm{OH}) \mathrm{D}_{3}$ concentrations below $50 \mathrm{nmol} / \mathrm{L}$ [80]. However, also $30 \mathrm{nmol} / \mathrm{L}$ or $75 \mathrm{nmol} / \mathrm{L}$ are used as cut-off values for vitamin D deficiency [77]. The cut-off value for optimal serum $25(\mathrm{OH}) \mathrm{D}_{3}$ is likely also depending on health outcomes of interest. Today, those cut-off values and coherent recommendations are based on bone health $[80,81]$. However, optimum levels could be different with respect to CRC. A dose-response analysis regarding vitamin D concentrations and CRC outcomes showed that $25(\mathrm{OH}) \mathrm{D}_{3}$ concentrations up to $80 \mathrm{nmol} / \mathrm{L}$ appeared to reduce all-cause mortality, while no additional beneficial effect was observed with concentrations $>80 \mathrm{nmol} / \mathrm{L}$ [19]. These results indicate an optimum level of $80 \mathrm{nmol} / \mathrm{L}$. Based on our results and previous results, we hypothesize that most health gain can be achieved by increasing deficient vitamin D concentrations to $75-80$ nmol/L.

\section{PRELIMINARY GUIDELINE 2: LOW-GRADE CHRONIC INFLAMMATION SHOULD BE TREATED BY IMPROVING VITAMIN D CONCENTRATIONS AND A MORE ANTI-INFLAMMATORY DIET.}

As mentioned before, higher levels of inflammatory markers were associated with worse CRC outcomes. Health care professionals should be aware of the negative effects of lowgrade chronic inflammation on health outcomes. In Chapter 6, we observed that patients with a higher level of chronic inflammation were more often overweight, less physically active and had lower $25(\mathrm{OH}) \mathrm{D}_{3}$ concentrations. Furthermore, in Chapter 7 , we observed that higher levels of inflammatory markers were associated with a more pro-inflammatory diet, characterized by a higher intake of refined grains and fast foods and a lower intake of fruit, vegetables and nuts. In addition, in our previous work (not part of this thesis) we observed that a better adherence to the World Cancer Research Fund / American Institute for Cancer Research recommendations for cancer prevention, which include guidelines on body weight, physical activity and a healthy diet, was associated with lower levels of IL6, IL8, TNF $\alpha$ and CRP [82]. Taken together, actively adopting a healthier lifestyle and optimizing $25(\mathrm{OH}) \mathrm{D}_{3}$ concentrations could probably decrease inflammation and improve prognosis of CRC. The effect of adopting a healthier lifestyle is probably going beyond cancer alone, as inflammation is also linked to cardiometabolic diseases which are common in CRC survivors $[37,38]$. To stimulate adherence to a healthy lifestyle during and after cancer treatment, nutrition and exercise programs guided by dieticians and lifestyle 
coaches are necessary, also during follow-up care. In addition, more personalized evidence-based guidelines for subgroups of CRC survivors are required to improve selfempowerment of CRC survivors.

\section{FUTURE RESEARCH}

For future research we will elaborate on three key aspects 1) the added value of tumour tissue, 2) intervention studies investigating the effect of increasing $25(\mathrm{OH}) \mathrm{D}_{3}$ concentrations in relation to $\mathrm{CRC}$ outcomes and 3) further unraveling the antiinflammatory effect of vitamin D and the diet.

Although this thesis adds to the evidence for a potential causal role of vitamin D in CRC prognosis, underlying mechanisms of vitamin D, inflammation and CRC outcomes should be further elucidated by investigating tumour tissue before firm conclusions can be drawn. In this thesis we investigated circulating $25(\mathrm{OH}) \mathrm{D}_{3}$ concentrations as well as circulating inflammatory markers. However, it is important to note that plasma markers of vitamin $D$ and inflammation may not exclusively reflect processes within the tumour and the tumour microenvironment. Measuring vitamin D status and inflammation in the tumour itself may provide a better understanding of the interaction between vitamin $D$ and inflammation in CRC prognosis. In addition, the association between vitamin D, inflammation and CRC outcomes is likely dependent on immune-related tumour characteristics [83, 84]. A study in 869 CRC patients observed a stronger association between predicted vitamin D concentrations and CRC survival in patients with lower peritumoural lymphocytic reaction compared to patients with a higher lymphocytic reaction [84]. Next to the effect of vitamin D on CRC outcomes, also the effect of the inflammatory potential of the diet on CRC outcomes may differ among molecular tumour subtypes with different degrees of immune responses [85]. Thus, an integrative evaluation of 1 ) circulating $25(\mathrm{OH}) \mathrm{D}_{3}$ concentrations and systemic inflammation, 2 ) detailed profiling of vitamin D, inflammation and host immunity in the tumour microenvironment and 3 ) factors influencing vitamin D concentrations and inflammation is important to extend our knowledge on underlying mechanisms regarding the complex interplay between exposures (e.g. vitamin D, nutrition) and tumour immune responses in relation to CRC prognosis. This molecular pathological epidemiological research approach $[86,87]$ is essential to further unravel such complex interrelationships.

In addition, trials with vitamin $\mathrm{D}$ supplementation considering actual $25(\mathrm{OH}) \mathrm{D}_{3}$ concentrations over time should be conducted. Until now the same dosage of vitamin D supplements was provided to all individuals in the intervention group independent of vitamin D status. Two randomized controlled trials investigated the effect of vitamin D supplementation on CRC mortality $[57,58]$. The AMATERASU trial, conducted in Japan, included stage I-III CRC patients [57], and the SUNSHINE trial, conducted in the USA, 
included patients with stage IV CRC [58]. CRC-specific mortality was included as a secondary outcome in some other trials with vitamin $D$ supplementation in the general population [59-62]. The dosage of vitamin D supplementation varied between 10-100 $\mu \mathrm{g} /$ day and the duration of the interventions between six months and seven years. Although most trials observed a beneficial effect of vitamin $D$ on progression-free and CRC-specific survival, no statistically significant associations were observed. However, the general population trials had limited power with respect to CRC-specific deaths as an outcome. Trials in CRC patients are needed, with a minimal follow-up of 5 years to obtain better estimates of recurrence and cancer-specific survival, as previous studies had a median follow-up time of 3.5 years [57] and 1.9 years [58]. In the proposed trials dosage and duration of vitamin D supplementations should be based on $25(\mathrm{OH}) \mathrm{D}_{3}$ concentrations rather than providing all participants in the intervention group the same dosage of vitamin D supplements. For example, a higher (starting) dosage should be given to patients with insufficient $25(\mathrm{OH}) \mathrm{D}_{3}$ concentrations, while patients with sufficient levels do not need a high starting dose. Considering the results of this thesis, also the combined exposure of vitamin $D$, magnesium and calcium in relation to $C R C$ recurrence and mortality should be further investigated first in observational studies. In addition, the source of magnesium and calcium (supplemental vs dietary) should be explored. Also inflammation as an underlying mechanism should be further investigated using a molecular pathological epidemiological research approach. Special attention should be given to cytokines known to be involved in tumour progression such as IL6, IL8 and TNF $\alpha 36,88]$, and to immune cell infiltration in tumour tissue [89] rather than on more general markers of inflammation, such as CRP.

\section{OVERALL CONCLUSION}

In the general introduction I ended the first paragraph with a couple of unanswered questions regarding the "black box" between vitamin D and CRC prognosis. Here I will come back to those questions to provide answers.

Is vitamin D casually related to CRC prognosis? Results of this thesis (and previous research) give a good indication for a causal relationship with all-cause mortality. The evidence is less clear for $C R C$ recurrence as research in this field is still limited. Caution is warranted in drawing conclusions about cause and effect based on the observational analyses in this thesis, conducted using data from prospective cohort studies. Clarifying underlying mechanisms and results of randomized controlled trials in future research will add the final pieces of evidence needed to answer this question.

Does vitamin D improve CRC prognosis by reducing inflammation? Results of this research provides some insights into the "black box" between vitamin D and CRC prognosis. Serum $25(\mathrm{OH}) \mathrm{D}_{3}$ concentrations over time are associated with a better cancer prognosis. In 
addition, $25(\mathrm{OH}) \mathrm{D}_{3}$ concentrations were associated with lower levels of inflammation markers, while lower levels of inflammation markers were associated with a better prognosis. Thus although results of this thesis give some indirect clues about inflammation as an underlying mechanisms, more research, for example investigating vitamin $D$ concentrations and inflammation within the tumour microenvironment, is needed to further unravel the role of inflammation.

Are vitamin D and inflammation associated with CRC recurrences? Based on the results of this thesis and previous research, we cannot conclude anything about the association between vitamin $D$ and recurrences. Data is still limited and results are conflicting. With respect to inflammation, higher levels of inflammation seem to increase the risk of CRC recurrence.

In conclusion, $25(\mathrm{OH}) \mathrm{D}_{3}$ concentrations and levels of inflammation markers were both associated with CRC outcomes. In addition, higher $25(\mathrm{OH}) \mathrm{D}_{3}$ concentrations were associated with lower levels of inflammation markers. Results of this thesis provide a foundation for a causal relationship between higher $25(\mathrm{OH}) \mathrm{D}_{3}$ concentrations and allcause mortality in CRC survivors. However, the role of vitamin D in cancer recurrence is still unclear. The anti-inflammatory effects of vitamin $D$ as one of the potential underlying mechanisms for its association with CRC outcomes should be further explored using a molecular pathological epidemiological research approach. The work of this thesis adds important knowledge to the field of nutrition and CRC survival. Ultimately, insights into the role of vitamin D and inflammation in CRC survival could lead to the development of guidelines about vitamin D for CRC patients, which may beneficially affect CRC prognosis. 


\section{REFERENCES}

[1] Mezawa H, Sugiura T, Watanabe M, Norizoe C, Takahashi D, Shimojima A, et al. Serum vitamin D levels and survival of patients with colorectal cancer: post-hoc analysis of a prospective cohort study. BMC Cancer. 2010;10:347.

[2] Fedirko V, Riboli E, Tjønneland A, Ferrari P, Olsen A, Bueno-de-Mesquita HB, et al. Prediagnostic 25hydroxyvitamin D, VDR and CASR polymorphisms, and survival in patients with colorectal cancer in western European populations. Cancer Epidemiology and Prevention Biomarkers. 2012;21:582-93.

[3] Zgaga L, Theodoratou E, Farrington SM, Din FV, Ooi LY, Glodzik D, et al. Plasma Vitamin D Concentration Influences Survival Outcome After a Diagnosis of Colorectal Cancer. Journal of Clinical Oncology. 2014;32:2430-9. [4] Maalmi H, Walter V, Jansen L, Chang-Claude J, Owen RW, Ulrich A, et al. Relationship of very low serum 25hydroxyvitamin D3 levels with long-term survival in a large cohort of colorectal cancer patients from Germany. European journal of epidemiology. 2017;32:961-71.

[5] Wesa KM, Segal NH, Cronin AM, Sjoberg DD, Jacobs GN, Coleton MI, et al. Serum 25-hydroxy vitamin D and survival in advanced colorectal cancer: a retrospective analysis. Nutrition and cancer. 2015;67:424-30.

[6] Touvier M, Deschasaux M, Montourcy M, Sutton A, Charnaux N, Kesse-Guyot E, et al. Determinants of vitamin D status in Caucasian adults: influence of sun exposure, dietary intake, sociodemographic, lifestyle, anthropometric, and genetic factors. Journal of Investigative Dermatology. 2015;135:378-88.

[7] Van Dam RM, Snijder MB, Dekker JM, Stehouwer CD, Bouter LM, Heine RJ, et al. Potentially modifiable determinants of vitamin D status in an older population in the Netherlands: the Hoorn Study-. The American journal of clinical nutrition. 2007;85:755-61.

[8] Brock K, Huang W-Y, Fraser D, Ke L, Tseng M, Stolzenberg-Solomon R, et al. Low vitamin D status is associated with physical inactivity, obesity and low vitamin $D$ intake in a large US sample of healthy middle-aged men and women. The Journal of steroid biochemistry and molecular biology. 2010;121:462-6.

[9] Larose TL, Chen Y, Camargo CA, Langhammer A, Romundstad P, Mai X-M. Factors associated with vitamin D deficiency in a Norwegian population: the HUNT Study. Journal of epidemiology and community health. 2014;68:165-70.

[10] Lips P, Schoor NM, Jongh RT. Diet, sun, and lifestyle as determinants of vitamin D status. Annals of the New York Academy of Sciences. 2014;1317:92-8.

[11] Churilla TM, Brereton HD, Klem M, Peters CA. Vitamin D deficiency is widespread in cancer patients and correlates with advanced stage disease: a community oncology experience. Nutrition and cancer. 2012;64:521-5.

[12] Chang PH, Pan YP, Fan CW, Tseng WK, Huang JS, Wu TH, et al. Pretreatment serum interleukin-1 $\beta$, interleukin-6, and tumor necrosis factor- $\alpha$ levels predict the progression of colorectal cancer. Cancer medicine. 2016;5:426-33.

[13] Rasic I, Radovic S, Aksamija G. Relationship between chronic inflammation and the stage and histopathological size of colorectal carcinoma. Medical Archives. 2016;70:104.

[14] Mangin M, Sinha R, Fincher K. Inflammation and vitamin D: the infection connection. Inflammation Research. 2014;63:803-19.

[15] Fakih MG, Trump DL, Johnson CS, Tian L, Muindi J, Sunga AY. Chemotherapy is linked to severe vitamin D deficiency in patients with colorectal cancer. International journal of colorectal disease. 2009;24:219.

[16] Isenring EA, Teleni L, Woodman RJ, Kimlin MG, Walpole E, Karapetis CS, et al. Serum vitamin D decreases during chemotherapy: an Australian prospective cohort study. Asia Pacific journal of clinical nutrition.

2018;27:962.

[17] Peterson DE, Bensadoun R-J, Roila F, Group ObotEGW. Management of oral and gastrointestinal mucositis: ESMO Clinical Practice Guidelines. Annals of Oncology. 2011;22:vi78-vi84.

[18] Vaughan-Shaw PG, Zgaga L, Ooi LY, Theodoratou E, Timofeeva M, Svinti V, et al. Low plasma vitamin D is associated with adverse colorectal cancer survival after surgical resection, independent of systemic inflammatory response. Gut. 2020;69:103-11. 
[19] Wu G, Xue M, Zhao Y, Han Y, Zhang S, Zhang J, et al. Low circulating 25-hydroxyvitamin D level is associated with increased colorectal cancer mortality: a systematic review and dose-response meta-analysis. Bioscience Reports. 2020;40.

[20] Deng X, Song Y, Manson JE, Signorello LB, Zhang SM, Shrubsole MJ, et al. Magnesium, vitamin D status and mortality: results from US National Health and Nutrition Examination Survey (NHANES) 2001 to 2006 and NHANES III. BMC medicine. 2013;11:187.

[21] Uwitonze AM, Razzaque MS. Role of magnesium in vitamin D activation and function. The Journal of the American Osteopathic Association 2018;118:181-9.

[22] Dai Q, Zhu X, Manson JE, Song Y, Li X, Franke AA, et al. Magnesium status and supplementation influence vitamin $D$ status and metabolism: results from a randomized trial. The American journal of clinical nutrition. 2018;108:1249-58.

[23] Reddy P, Edwards LR. Magnesium supplementation in vitamin D deficiency. American journal of therapeutics. 2019;26:e124-e32.

[24] Krishnan AV, Feldman D. Mechanisms of the anti-cancer and anti-inflammatory actions of vitamin D. Annual review of pharmacology and toxicology. 2011;51:311-36.

[25] Xie DD, Chen YH, Xu S, Zhang C, Wang DM, Wang H, et al. Low vitamin D status is associated with inflammation in patients with prostate cancer. Oncotarget. 2017;8:22076-85.

[26] Vaughan-Shaw PG, Buijs LF, Blackmur JP, Theodoratou E, Zgaga L, Din FVN, et al. The effect of vitamin D supplementation on survival in patients with colorectal cancer: systematic review and meta-analysis of randomised controlled trials. British Journal of Cancer. 2020.

[27] Hopkins MH, Owen J, Ahearn T, Fedirko V, Flanders WD, Jones DP, et al. Effects of supplemental vitamin D and calcium on biomarkers of inflammation in colorectal adenoma patients: a randomized, controlled clinical trial. Cancer prevention research. 2011;4:1645-54.

[28] Seruga B, Zhang H, Bernstein L, Tannock IF. Cytokines and their relationship to the symptoms and outcome of cancer. Nature Reviews Cancer. 2008;8:887.

[29] Xu J, Ye Y, Zhang H, Szmitkowski M, Mäkinen M, Li P, et al. Diagnostic and prognostic value of serum interleukin-6 in colorectal cancer. Medicine. 2016;95.

[30] Knupfer H, Preiss R. Serum interleukin-6 levels in colorectal cancer patients--a summary of published results. International journal of colorectal disease. 2010;25:135-40.

[31] Abtahi S, Davani F, Mojtahedi Z, Hosseini SV, Bananzadeh A, Ghaderi A. Dual association of serum interleukin-10 levels with colorectal cancer. Journal of cancer research and therapeutics. 2017;13:252.

[32] Stanilov N, Miteva L, Dobreva Z, Stanilova S. Colorectal cancer severity and survival in correlation with tumour necrosis factor-alpha. Biotechnology \& Biotechnological Equipment. 2014;28:911-7.

[33] Xia W, Chen W, Zhang Z, Wu D, Wu P, Chen Z, et al. Prognostic value, clinicopathologic features and diagnostic accuracy of interleukin-8 in colorectal cancer: a meta-analysis. PloS one. 2015;10:e0123484.

[34] Woo HD, Kim K, Kim J. Association between preoperative C-reactive protein level and colorectal cancer survival: a meta-analysis. Cancer Causes \& Control. 2015;26:1661-70.

[35] Greten FR, Grivennikov SI. Inflammation and Cancer: Triggers, Mechanisms, and Consequences. Immunity. 2019;51:27-41.

[36] West NR, McCuaig S, Franchini F, Powrie F. Emerging cytokine networks in colorectal cancer. Nature Reviews Immunology. 2015;15:615-29.

[37] Alfano CM, Peng J, Andridge RR, Lindgren ME, Povoski SP, Lipari AM, et al. Inflammatory cytokines and comorbidity development in breast cancer survivors versus noncancer controls: evidence for accelerated aging? Journal of Clinical Oncology. 2017;35:149.

[38] Lopez-Candales A, Burgos PMH, Hernandez-Suarez DF, Harris D. Linking chronic inflammation with cardiovascular disease: from normal aging to the metabolic syndrome. Journal of nature and science. $2017 ; 3$.

[39] Wiseman MJ. Nutrition and cancer: prevention and survival. British Journal of Nutrition. 2018:1-7.

[40] Galland L. Diet and inflammation. Nutrition in Clinical Practice. 2010;25:634-40.

[41] Barbaresko J, Koch M, Schulze MB, Nöthlings U. Dietary pattern analysis and biomarkers of low-grade inflammation: a systematic literature review. Nutrition reviews. 2013;71:511-27. 
[42] Deng FE, Shivappa N, Tang Y, Mann JR, Hebert JR. Association between diet-related inflammation, all-cause, all-cancer, and cardiovascular disease mortality, with special focus on prediabetics: findings from NHANES III. European journal of nutrition. 2017;56:1085-93.

[43] Ratjen I, Shivappa N, Schafmayer C, Burmeister G, Nothlings U, Hampe J, et al. Association between the dietary inflammatory index and all-cause mortality in colorectal cancer long-term survivors. International journal of cancer Journal international du cancer. 2019;144:1292-301.

[44] Zheng J, Tabung FK, Zhang J, Murphy EA, Shivappa N, Ockene JK, et al. Post-cancer diagnosis dietary inflammatory potential is associated with survival among women diagnosed with colorectal cancer in the Women's Health Initiative. European journal of nutrition. 2019.

[45] Tabung FK, Steck SE, Ma Y, Liese AD, Zhang J, Caan B, et al. The association between dietary inflammatory index and risk of colorectal cancer among postmenopausal women: results from the Women's Health Initiative. Cancer Causes \& Control. 2015;26:399-408.

[46] Fedak KM, Bernal A, Capshaw ZA, Gross S. Applying the Bradford Hill criteria in the 21st century: how data integration has changed causal inference in molecular epidemiology. Emerging themes in epidemiology.

2015;12:14.

[47] Yang L, Chen H, Zhao M, Peng P. Prognostic value of circulating vitamin D binding protein, total, free and bioavailable 25-hydroxy vitamin $D$ in patients with colorectal cancer. Oncotarget. 2017;8:40214-21.

[48] Zhang H, Ahn J, Yu K. Comparing statistical methods for removing seasonal variation from vitamin D measurements in case-control studies. Statistics and its interface. 2011;4:85-93.

[49] Cleveland WS, Devlin SJ. Locally Weighted Regression: An Approach to Regression Analysis by Local Fitting. Journal of the American Statistical Association. 1988;83:596-610.

[50] Davies NM, Holmes MV, Davey Smith G. Reading Mendelian randomisation studies: a guide, glossary, and checklist for clinicians. Bmj. 2018;362:k601.

[51] Theodoratou E, Palmer T, Zgaga L, Farrington SM, McKeigue P, Din FV, et al. Instrumental variable estimation of the causal effect of plasma 25-hydroxy-vitamin $D$ on colorectal cancer risk: a mendelian randomization analysis. PloS one. 2012;7:e37662.

[52] He Y, Timofeeva M, Farrington SM, Vaughan-Shaw P, Svinti V, Walker M, et al. Exploring causality in the association between circulating 25 -hydroxyvitamin $\mathrm{D}$ and colorectal cancer risk: a large Mendelian randomisation study. BMC medicine. 2018;16:142.

[53] Dimitrakopoulou VI, Tsilidis KK, Haycock PC, Dimou NL, Al-Dabhani K, Martin RM, et al. Circulating vitamin D concentration and risk of seven cancers: Mendelian randomisation study. Bmj. 2017;359.

[54] Hiraki LT, Qu C, Hutter CM, Baron JA, Berndt SI, Bézieau S, et al. Genetic predictors of circulating 25hydroxyvitamin $d$ and risk of colorectal cancer. Cancer Epidemiology and Prevention Biomarkers. 2013;22:203746.

[55] Chandler PD, Tobias DK, Wang L, Smith-Warner SA, Chasman DI, Rose L, et al. Association between Vitamin D Genetic Risk Score and Cancer Risk in a Large Cohort of U.S. Women. Nutrients. 2018;10:55.

[56] Keum N, Lee DH, Greenwood DC, Manson JE, Giovannucci E. Vitamin D supplementation and total cancer incidence and mortality: a meta-analysis of randomized controlled trials. Annals of Oncology. 2019;30:733-43.

[57] Urashima M, Ohdaira H, Akutsu T, Okada S, Yoshida M, Kitajima M, et al. Effect of Vitamin D Supplementation on Relapse-Free Survival Among Patients With Digestive Tract Cancers: The AMATERASU Randomized Clinical TrialEffect of Vitamin D Supplementation on Relapse-Free Survival in Patients With Digestive Tract CancersEffect of Vitamin D Supplementation on Relapse-Free Survival in Patients With Digestive Tract Cancers. JAMA : the journal of the American Medical Association. 2019;321:1361-9.

[58] Ng K, Nimeiri HS, McCleary NJ, Abrams TA, Yurgelun MB, Cleary JM, et al. Effect of High-Dose vs StandardDose Vitamin D3 Supplementation on Progression-Free Survival Among Patients With Advanced or Metastatic Colorectal Cancer: The SUNSHINE Randomized Clinical TrialEffect of High-vs Standard-Dose Vitamin D3 on Progression-Free Survival of Colorectal CancerEffect of High- vs Standard-Dose Vitamin D3 on Progression-Free Survival of Colorectal Cancer. JAMA : the journal of the American Medical Association. 2019;321:1370-9. 
[59] Trivedi DP, Doll R, Khaw KT. Effect of four monthly oral vitamin D3 (cholecalciferol) supplementation on fractures and mortality in men and women living in the community: randomised double blind controlled trial. Bmj. 2003;326:469.

[60] Wactawski-Wende J, Kotchen JM, Anderson GL, Assaf AR, Brunner RL, O'Sullivan MJ, et al. Calcium plus vitamin $D$ supplementation and the risk of colorectal cancer. The New England journal of medicine. 2006;354:684-96.

[61] Avenell A, MacLennan GS, Jenkinson DJ, McPherson GC, McDonald AM, Pant PR, et al. Long-term follow-up for mortality and cancer in a randomized placebo-controlled trial of vitamin $D(3)$ and/or calcium (RECORD trial). The Journal of clinical endocrinology and metabolism. 2012;97:614-22.

[62] Chandler PD, Chen WY, Ajala ON, Hazra A, Cook N, Bubes V, et al. Effect of Vitamin D3 Supplements on Development of Advanced Cancer: A Secondary Analysis of the VITAL Randomized Clinical Trial. JAMA Netw Open. 2020;3:e2025850.

[63] Richiardi L, Bellocco R, Zugna D. Mediation analysis in epidemiology: methods, interpretation and bias. International journal of epidemiology. 2013;42:1511-9.

[64] van Harten-Gerritsen AS, Balvers MG, Witkamp RF, Kampman E, van Duijnhoven FJ. Vitamin D, inflammation and colorectal cancer progression: A review of mechanistic studies and future directions for epidemiological studies. Cancer Epidemiology and Prevention Biomarkers. 2015:cebp. 0601.2015.

[65] Brenner DR, Scherer D, Muir K, Schildkraut J, Boffetta P, Spitz MR, et al. A Review of the Application of Inflammatory Biomarkers in Epidemiologic Cancer Research. Cancer epidemiology, biomarkers \& prevention : a publication of the American Association for Cancer Research, cosponsored by the American Society of Preventive Oncology. 2014;23:1729-51.

[66] de Jager W, Bourcier K, Rijkers GT, Prakken BJ, Seyfert-Margolis V. Prerequisites for cytokine measurements in clinical trials with multiplex immunoassays. BMC immunology. 2009;10:52.

[67] Friedenreich CM, Shaw E, Neilson HK, Brenner DR. Epidemiology and biology of physical activity and cancer recurrence. Journal of Molecular Medicine. 2017;95:1029-41.

[68] Elferink MAG, de Jong KP, Klaase JM, Siemerink EJ, de Wilt JHW. Metachronous metastases from colorectal cancer: a population-based study in North-East Netherlands. International journal of colorectal disease.

2015;30:205-12.

[69] Saif MW, Chu E. Biology of Colorectal Cancer. The Cancer Journal. 2010;16:196-201.

[70] Krishnan AV, Feldman D. Mechanisms of the anti-cancer and anti-inflammatory actions of vitamin D. Annual review of pharmacology and toxicology. 2011;51:311-36.

[71] van den Ouweland JM, Beijers AM, van Daal H. Overestimation of 25-hydroxyvitamin D3 by increased ionisation efficiency of 3-epi-25-hydroxyvitamin D3 in LC-MS/MS methods not separating both metabolites as determined by an LC-MS/MS method for separate quantification of 25-hydroxyvitamin D3, 3-epi-25hydroxyvitamin D3 and 25-hydroxyvitamin D2 in human serum. Journal of Chromatography B. 2014;967:195-202. [72] van den Ouweland JM, Vogeser M, Bacher S. Vitamin D and metabolites measurement by tandem mass spectrometry. Reviews in endocrine \& metabolic disorders. 2013;14:159-84.

[73] van den Ouweland JMW, Beijers AM, Demacker PNM, van Daal H. Measurement of 25-OH-vitamin D in human serum using liquid chromatography tandem-mass spectrometry with comparison to radioimmunoassay and automated immunoassay. J Chromatogr B Analyt Technol Biomed Life Sci. 2010;878:1163-8.

[74] Brouwer NP, Bos AC, Lemmens VE, Tanis PJ, Hugen N, Nagtegaal ID, et al. An overview of 25 years of incidence, treatment and outcome of colorectal cancer patients. International journal of cancer. 2018;143:275866.

[75] Counsel DH. Dossier Evaluatie van de voedingsnormen voor vitamine D. 2012.

[76] EFSA Panel on Dietetic Products N, Allergies. Dietary reference values for vitamin D. EFSA Journal. 2016;14:e04547.

[77] Alshahrani F, Aljohani N. Vitamin D: Deficiency, Sufficiency and Toxicity. Nutrients. 2013;5:3605-16.

[78] Chel V, Elders P, Tuijp M, van den Berg H, Drongelen K, Siedenburg R, et al. Vitamine D-suppletie bij ouderen: advies en praktijk. Nederlands Tijdschrift voor Geneeskunde. 2013;157. 
[79] Robien K, Oppeneer SJ, Kelly JA, Hamilton-Reeves JM. Drug-vitamin D interactions: a systematic review of the literature. Nutrition in Clinical Practice. 2013;28:194-208.

[80] Holick MF, Binkley NC, Bischoff-Ferrari HA, Gordon CM, Hanley DA, Heaney RP, et al. Evaluation, treatment, and prevention of vitamin D deficiency: an Endocrine Society clinical practice guideline. The Journal of Clinical Endocrinology \& Metabolism. 2011;96:1911-30.

[81] Heaney RP. Vitamin D and calcium interactions: functional outcomes-. The American journal of clinical nutrition. 2008;88:541S-4S.

[82] Wesselink E, van Baar H, van Zutphen M, Tibosch M, Kouwenhoven EA, Keulen ETP, et al. Inflammation Is a Mediating Factor in the Association between Lifestyle and Fatigue in Colorectal Cancer Patients. Cancers. 2020;12.

[83] Punt CJ, Koopman M, Vermeulen L. From tumour heterogeneity to advances in precision treatment of colorectal cancer. Nature reviews Clinical oncology. 2017;14:235-46.

[84] Hamada T, Liu L, Nowak JA, Mima K, Cao Y, Ng K, et al. Vitamin D status after colorectal cancer diagnosis and patient survival according to immune response to tumour. European journal of cancer. 2018;103:98-107.

[85] Liu L, Nishihara R, Qian ZR, Tabung FK, Nevo D, Zhang X, et al. Association Between Inflammatory Diet Pattern and Risk of Colorectal Carcinoma Subtypes Classified by Immune Responses to Tumor. Gastroenterology. 2017;153:1517-30.e14.

[86] Ogino S, Campbell PT, Nishihara R, Phipps Al, Beck AH, Sherman ME, et al. Proceedings of the second international molecular pathological epidemiology (MPE) meeting. Cancer Causes \& Control. 2015;26:959-72.

[87] Ogino S, Chan AT, Fuchs CS, Giovannucci E. Molecular pathological epidemiology of colorectal neoplasia: an emerging transdisciplinary and interdisciplinary field. Gut. 2011;60:397-411.

[88] Li J, Huang L, Zhao H, Yan Y, Lu J. The Role of Interleukins in Colorectal Cancer. International Journal of Biological Sciences. 2020;16:2323-39.

[89] Pagès F, Mlecnik B, Marliot F, Bindea G, Ou F-S, Bifulco C, et al. International validation of the consensus Immunoscore for the classification of colon cancer: a prognostic and accuracy study. The Lancet. 2018;391:212839. 

SUMMARY / SAMENVATTING 
Colorectal cancer (CRC) accounts for one in ten cancer cases and deaths worldwide, and the incidence of CRC is still increasing. The number of CRC survivors is further increasing due to population screening programs and better treatments, which have increased cancer survival rates. Cancer recurrence is a major concern for CRC survivors and an important contributor to mortality in this growing population. Therefore, it is necessary to optimize long-term survival in CRC patients. Vitamin D is a potentially interesting compound in this respect, as high compared to low concentrations of vitamin D at diagnosis are associated with higher survival rates in CRC survivors. It is hypothesized that vitamin D exerts its beneficial effect on CRC survival due to its anti-inflammatory potential. Nevertheless, many questions remain unanswered regarding the "black box" between vitamin D and CRC survival. Therefore, the aim of this study was to investigate the relationship between vitamin $D$, inflammation and their interplay in relation to $C R C$ recurrence and all-cause mortality in two prospective cohort studies in CRC patients in the Netherlands; the COLON study and the EnCoRe study.

The relative importance of dietary and lifestyle factors in conjunction with clinical factors on vitamin D status in CRC patients is presently unknown. Therefore, we evaluated which clinical characteristics in conjunction with demographic and lifestyle factors are associated with serum 25-hydroxyvitamin $\mathrm{D}_{3}\left(25(\mathrm{OH}) \mathrm{D}_{3}\right)$ concentrations at diagnosis and six months later in chapter 2 . The main determinants of vitamin $D$ status as well as changes in concentrations over time were the use of vitamin $\mathrm{D}$ supplementations and receiving chemotherapy. Vitamin D supplement use was associated with higher $25(\mathrm{OH}) \mathrm{D}_{3}$ concentrations. Receiving chemotherapy was associated with lower $25(\mathrm{OH}) \mathrm{D}_{3}$ concentrations and a steeper decrease in $25(\mathrm{OH}) \mathrm{D}_{3}$ concentrations in the first six months after diagnosis. Other determinants of low vitamin $\mathrm{D}$ concentrations were a more advanced stage of disease, a higher body mass index (BMI) and lower physical activity levels.

Higher concentrations of $25(\mathrm{OH}) \mathrm{D}_{3}$ at diagnosis were previously associated with a lower all-cause mortality risk in CRC patients. However, in these previous studies the interaction with nutrients involved in vitamin D metabolism (i.e. magnesium en calcium) were not taken into account. Therefore, vitamin $D$, magnesium and calcium in relation to CRC outcomes was investigated in chapter 3. Moreover, the interaction between vitamin $D$, magnesium and calcium in relation to CRC was examined. Lower concentrations of vitamin $D$ (severe deficient < 30 nmol/L vs sufficient $(50-75 \mathrm{nmol} / \mathrm{L}$ ) seemed to be associated with a higher risk of all-cause mortality. Furthermore, an inverse association between magnesium intake, but not calcium intake, and all-cause mortality was observed. The lowest risk of all-cause mortality was observed in patients with sufficient vitamin D levels as well as a high magnesium intake, suggesting a synergistic interaction between vitamin $D$ and magnesium. No interaction between vitamin $D$ and calcium was observed. 
In addition, $25(\mathrm{OH}) \mathrm{D}_{3}$ concentrations are likely to change over time, which could also affect health outcomes in CRC patients. In chapter $\mathbf{4}$ of this thesis the association between trajectories of vitamin D concentrations over time and CRC outcomes was assessed. Around $20 \%$ of all CRC patients had consistent deficient vitamin D levels from diagnosis until two years after diagnosis, $39 \%$ had consistently sufficient levels, while $20 \%$ had increasing levels and $23 \%$ a temporary drop during treatment. Patients characterized by one of the three trajectories with sufficient levels of vitamin $D$ two years after diagnosis had a lower risk of all-cause mortality compared to patients with consistent deficient levels. No associations for recurrence were observed.

One of the potential mechanisms by which vitamin D exerts beneficial effects in cancer is by its anti-inflammatory properties. Therefore, we investigated the association between vitamin $\mathrm{D}$ concentrations and concentrations of inflammatory markers over time in chapter 5 . Higher serum $25(\mathrm{OH}) \mathrm{D}_{3}$ levels at diagnosis, six months and two year after diagnosis as well as over time were associated with lower interleukin 6 (IL6) levels and a combined inflammatory z-score. Serum $25(\mathrm{OH}) \mathrm{D}_{3}$ levels were not associated with Creactive protein, IL8, IL10 and Tumour Necrosis Factor $\alpha$ (TNF $\alpha$ ) levels in plasma. These results indicate that vitamin $D$ could potentially lower inflammation marker levels, however, further intervention studies, investigating the effect of increasing vitamin $D$ levels on inflammatory mediators in CRC patients are needed.

The association between pre-operative and post-operative concentrations of inflammation markers and CRC outcomes, while taking into account lifestyle factors, was investigated in chapter 6 . Higher pre-operative as well as post-operative CRP levels were associated with a higher risk of recurrence. Higher pre-operative as well as post-operative IL8 and a combined inflammatory z-score were associated with a higher risk of all-cause mortality.

Diet has an effect on inflammation and subsequent health status. The inflammatory potential of the diet was associated with all-cause mortality in CRC patients in previous studies. However, it is unknown whether the inflammatory potential of the diet is also associated with cancer recurrence. Therefore, we investigated the association between the inflammatory potential of the diet and CRC outcomes in chapter 7 . The inflammatory potential of the diet was assessed using the Adapted Dietary Inflammatory Index (ADII). Higher ADII scores were associated with higher levels of TNF $\alpha$, CRP and the inflammatory $z$-score at diagnosis and six months after diagnosis, but not with recurrence and all-cause mortality.

Taken together, the findings of this thesis suggest that higher vitamin D concentrations, especially in combination with magnesium, are associated with better CRC outcomes. In addition, inflammation is one of the potential underlying mechanisms, as higher 
concentrations of vitamin D were associated with lower levels of inflammation markers, while higher levels of inflammation markers itself were associated with worse CRC outcomes. In this thesis, no associations were observed between the inflammatory potential of the diet and CRC outcomes.

An important question to answer is whether vitamin $D$ is causally related to CRC outcomes. Higher $25(\mathrm{OH}) \mathrm{D}_{3}$ concentrations were moderately strong and consistently associated with a lower risk of mortality in CRC patients in epidemiological studies. In addition, a dose-response relationship was observed and a plausible biological pathway was described. Although the afore mentioned evidence provides a good indication for a causal relationship between vitamin $\mathrm{D}$ and all-cause mortality, before conclusions can be drawn about causality 1) underlying mechanisms should be further clarified and 2) intervention studies investigating the effect of increasing $25(\mathrm{OH}) \mathrm{D}_{3}$ concentrations in relation to CRC outcomes should be conducted. In addition, while inflammatory cytokines are the most reliable markers of inflammation and serum $25(\mathrm{OH}) \mathrm{D}_{3}$ concentrations the most reliable marker of vitamin $D$ status in the setting of a large prospective cohort study, circulating concentrations of vitamin $D$ and inflammation may not exclusively reflect processes within the tumour and the tumour microenvironment. Measuring vitamin $D$ status and inflammation in the tumour itself may provide a better understanding of the interaction between vitamin $\mathrm{D}$ and inflammation in CRC prognosis.

To conclude, results of this thesis provide a foundation for a causal relationship between higher 25(OH)D3 concentrations and all-cause mortality in CRC patients. However, the role of vitamin $D$ in cancer recurrence is still unclear. The anti-inflammatory effects of vitamin $D$ as one of the potential underlying mechanisms for its association with CRC outcomes should be further explored using a molecular pathological epidemiological research approach. 


\section{Samenvatting voor hen die een voorkeur hebben voor de Nederlandse taal}

Het aantal patiënten dat dikke darmkanker overleeft stijgt. Dit komt doordat de diagnose tegenwoordig vaker in een vroeger stadium wordt vastgesteld, mede dankzij het bevolkingsonderzoek en doordat de behandelmogelijkheden blijven verbeteren. Veel mensen die dikke darmkanker hebben gehad maken zich zorgen over de terugkeer van de tumor. Dit is het geval bij ongeveer $30-40 \%$ van de patiënten die curatief behandeld zijn. Het is belangrijk om overleving van darmkanker te verbeteren. Vitamine $D$ is een mogelijk geschikte kandidaat hiervoor. Eerder onderzoek heeft aangetoond dat hogere vitamine D concentraties in het bloed bij diagnose zijn gelinkt aan een betere overleving van darmkanker. Een van de mogelijke onderliggende mechanismen hiervoor is het ontstekingsremmende effect van vitamine D. Daarom onderzoeken wij in dit proefschrift hoe vitamine $D$ en inflammatie samenhangen met elkaar en met de terugkeer en overleving van darmkanker in twee grote studies in darmkankerpatiënten, de COLON studie en de EnCoRe studie.

We weten dat bepaalde eigenschappen en bepaald gedrag van mensen samenhangen met vitamine D concentraties. Zo zijn vitamine D concentraties vaak lager in ouderen en mensen met overgewicht. Vitamine D concentraties zijn juist hoger in mensen die meer buiten sporten of vitamine D supplementen gebruiken. We weten niet hoe darmkanker en de behandeling van darmkanker vitamine D concentraties beïnvloeden. Daarom hebben we in hoofdstuk 2 van dit proefschrift onderzocht welke factoren samenhangen met vitamine $\mathrm{D}$ concentraties. De factoren die het sterkst bijdroegen aan vitamine $\mathrm{D}$ concentraties bij diagnoses, 6 maanden na diagnose en aan de verandering over tijd, waren het gebruik van vitamine $D$ supplementen en het krijgen van chemotherapie. Patiënten die vitamine $D$ supplementen gebruikten hadden vaker hogere vitamine $D$ concentraties. Patiënten die chemotherapie kregen hadden vaker lagere concentraties en vitamine $\mathrm{D}$ concentraties namen ook sterker af over de tijd. Andere factoren die samenhingen met lage vitamine $D$ concentraties waren een verder gevorderd ziektestadium, overgewicht en weinig lichamelijke activiteit.

Hogere concentraties van vitamine $D$ ten tijde van de diagnose van darmkanker hangen samen met een betere overleving onder darmkanker patiënten. In eerdere studies is er echter nooit gekeken naar de interactie met andere nutriënten die het metabolisme van vitamine $D$ beïnvloeden, zoals magnesium en calcium. Daarom hebben wij onderzocht in hoofdstuk 3 hoe vitamine D, magnesium en calcium op zichzelf samenhangen met uitkomstmaten zoals de terugkeer van kanker en overleving in darmkanker patiënten. Daarnaast hebben we ook gekeken of er een interactie was tussen de drie nutriënten in 
relatie tot darmkanker uitkomstmaten. Een slechtere vitamine D status (veel te lage concentraties $<30 \mathrm{nmol} / \mathrm{L}$ ten opzichte van adequate concentraties $(50-75 \mathrm{nmol} / \mathrm{L}) \mathrm{hing}$ samen met een groter risico op overlijden. Een hogere magnesium inname leek samen te hangen met een lager risico op overlijden. We zagen geen verbanden tussen calcium inname en risico op overlijden. De beste overlevingskansen zagen we in patiënten die zowel een adequate vitamine $D$ status als een magnesium-rijke voeding hadden. Magnesium rijke producten zijn donker groene bladgroenten, volkoren granen, noten en peulvruchten. Wij zagen geen interacties tussen de drie nutriënten in relatie tot de terugkeer van kanker.

Daarnaast is het heel waarschijnlijk dat vitamine D concentraties veranderen over tijd. Die veranderingen over tijd hebben mogelijk invloed op gezondheidsuitkomsten onder darmkankerpatiënten. Daarom hebben we in hoofdstuk 4 onderzocht hoe veranderingen van vitamine D over tijd samenhangen met darmkanker uitkomstmaten. Wij zagen dat ongeveer $20 \%$ van alle darmkanker patiënten altijd te lage vitamine D concentraties had, dat wil zeggen vanaf diagnose tot twee jaar na diagnose. Ongeveer $40 \%$ van de darmkankerpatiënten had altijd een adequate vitamine $D$ status. Bij $20 \%$ van de patiënten namen vitamine $D$ concentraties toe over tijd en bij $23 \%$ namen de vitamine $D$ concentraties af tijdens de behandeling en stegen daarna weer. Patiënten die adequate vitamine $D$ concentraties hadden 2 jaar na diagnose hadden een betere overlevingskans vergeleken met de patiënten die altijd te lage vitamine $D$ concentraties hadden. Voor de terugkeer van kanker zagen we geen verbanden.

Vitamine D heeft ontstekingsremmende eigenschappen en zou dus een mogelijk middel zijn om ontstekingswaarden in het bloed te verlagen. Daarom hebben we in hoofdstuk 5 onderzocht hoe vitamine D samenhangt met ontstekingsmarkers zoals C-reactief proteïne, interleukine 6 en interleukine 8. Wij zagen dat hogere vitamine D concentraties gerelateerd waren met lagere concentraties van interleukine 6 , een stofje wat een belangrijke rol speelt in het verloop van darmkanker.

In hoofdstuk 6 hebben we de associatie tussen concentraties van ontstekingsmarkers voor en na de operatie in relatie tot darmkanker uitkomstmaten onderzocht. Hierbij hebben we ook rekening gehouden met leefstijl factoren die mogelijk ontsteking en/of darmkanker uitkomstmaten beïnvloeden. We zagen dat hogere CRP concentraties, samenhingen met een hoger risico op de terugkeer van kanker. Hogere interleukine 8 concentraties en een gecombineerde score van alle gemeten ontstekingsmarkers hingen samen met een hoger risico op overlijden. 
Voeding heeft mogelijk een effect op chronische ontsteking in ons bloed en daarmee samenhangend onze gezondheid. Eerdere studies hebben onderzocht hoe de voeding samenhangt met ontstekingswaarden. Op basis hiervan is een score ontwikkeld die aangeeft in welke mate de voeding ontsteking beïnvloedt of in andere woorden die de "inflammatoire potentie van de voeding" weergeeft. Een hogere score (pro-inflammatoir) betekent dat een bepaald voedingspatroon ontsteking stimuleert. Eerdere studies hebben aangetoond dat een meer pro-inflammatoire voeding is gelinkt aan een slechtere overlevingskans. We weten nog niet of de inflammatoire potentie van onze voeding ook samenhangt met de terugkeer van kanker. Dit hebben we onderzocht in hoofdstuk 7 . We zagen een verband tussen voeding en een aantal ontstekingsmarkers, maar we zagen geen verband tussen de inflammatoire potentie van onze voeding en het risico op de terugkeer van de kanker of overleving.

Alles tezamen suggereren de bevindingen van dit proefschrift dat vitamine $D$, met name in combinatie met magnesium, gerelateerd is aan een lager risico op overlijden bij darmkanker patiënten. Daarnaast is ontsteking een van de mogelijke mechanismen die het verband tussen vitamine $D$ en darmkanker uitkomstmaten verklaart. Hogere vitamine $D$ concentraties hangen samen met een betere overleving en met lagere concentraties van ontstekingsmarkers. Lagere concentraties van ontstekingsmarkers zijn gelinkt aan een betere overleving. In dit proefschrift vonden we geen verbanden tussen de inflammatoire potentie van onze voeding en de terugkeer en overleving van darmkanker.

Een belangrijke vraag om te beantwoorden is of het verband tussen vitamine $D$ en darmkanker uitkomstmaten oorzakelijk is. Vitamine $D$ is in deze en andere studies gelinkt aan een lager risico op overlijden. Daarnaast is er een dosis-respons relatie (hoe hogere concentraties hoe beter de overleving) gevonden en is er bovendien sprake van een aannemelijk onderliggend biologisch mechanisme. Toch kunnen we nu nog geen sterke conclusies trekken met betrekken tot een oorzakelijk verband. Daarvoor zijn eerst interventie studies met vitamine D supplementen bij darmkanker patiënten nodig. Daarnaast zal in toekomstig onderzoek het meten van vitamine $D$ en ontsteking in de tumor zelf, vergeleken met het meten in bloed, meer inzicht geven in de relatie en interactie tussen vitamine $D$, ontsteking en het verloop van darmkanker.

Concluderend geven de resultaten van dit proefschrift een basis voor een oorzakelijk verband tussen hogere vitamine $D$ concentraties en overleving van darmkankerpatiënten. De rol die vitamine D mogelijk speelt in de terugkeer van kanker is nog steeds onduidelijk. Of vitamine D overleving na darmkanker positief beïnvloedt door zijn 
ontstekingsremmende eigenschappen moet nog verder onderzocht worden, bijvoorbeeld door ook te kijken naar vitamine $D$ en ontsteking in de tumor zelf. 


Acknowledgements

Dankwoord 
Terugkijkend op mijn PhD zie ik pas echt wat een geweldige tijd het is geweest. Ik heb er enorm van genoten en ben ik nu best trost op het resultaat. Dit resultaat had er niet kunnen zijn zonder de hulp en steun van vele anderen. ledereen die hieraan meegewerkt heeft, wil ik ontzettend bedanken en een aantal mensen in het bijzonder.

Allereerst de deelnemers van de COLON en de EnCoRe studies. Hartelijk dank allemaal voor jullie vrijwillige deelname, het afgeven van bloedsamples en het invullen van alle (veel te lange) vragenlijsten! Zonder jullie deelname aan deze studies zou dit promotietraject niet mogelijk zijn geweest.

Ellen, jij was degene die mij al weer heel wat jaartjes geleden enthousiast hebt gemaakt voor het onderzoek. Op dat moment had ik nooit verwacht bij jou mijn promotieonderzoek te mogen doen en ik heb daar geen seconde spijt van gehad. Dankjewel voor je frisse en kritische blik op mijn werk en de positieve prikkelende manier waarop je mij stimuleerde het beste uit mezelf en mijn onderzoek te halen. Dank je wel ook voor je steun en vertrouwen de afgelopen jaren.

Fränzel, een promotietraject met jou als begeleider is gewoon een feest. Ik had het niet beter kunnen treffen! Ik vond het super dat je me helemaal vrij mijn pad liet kiezen in mijn onderzoek en me stimuleerde om naast mijn promotietraject ook bij andere projecten betrokken te zijn. Bedankt dat ik altijd bij je terecht kon met vragen en bedankt voor de overleggen die naast nuttig ook vaak heel gezellig waren (en soms wel heel erg uitliepen).

Martijn, als ik mijn stukken rondstuurde voor feedback was jij altijd de eerste die reageerde. Dank je wel voor je betrokkenheid en je opbouwende feedback!

Matty, Renger, Michiel en Hans ook jullie zijn vanaf dag 1 (en eigenlijk al daarvoor) betrokken geweest bij mijn promotietraject. Ik heb jullie input vanuit jullie eigen invalshoek in de verschillende fasen heel erg gewaardeerd!

I would like to thank the members of the thesis committee, Prof. Dr. Edward L. Giovannucci, Prof. Dr. Lisette C.P.G.M. de Groot, Prof. Dr. Miriam Koopman, and Prof. Dr. Huub F.J. Savelkoul for your effort in reading and evaluating my thesis.

Dieuwertje, we hebben elkaar leren kennen toen ik mijn MSc thesis bij jou schreef. Afgelopen jaren heb ik met super veel plezier met je samengewerkt. Je bent een fantastische collega en ik hoop dat we nog heel veel jaren blijven samen werken!

Moniek, Harm en Anne de afgelopen jaren heb ik met veel plezier samen met jullie als mede-PhDers op de COLON studie gewerkt, bedankt daarvoor. Ik wil ook de andere 228 
(ex) members van het "COLON / Voeding en kanker clubje" (Anne-Sophie, Nynke, Merel, Ilse, Jesca, Renate, Koen, Judith, Klaske) van harte bedanken voor de goede sfeer en de prettige samenwerking! Een speciaal bedankje voor Koen voor het maken van een profielfoto voor in dit boekje. Joeri dank je wel voor het opzetten van een COLON database. Hendriek and João, bedankt voor jullie adviezen op het gebied van statistiek en data-analyse. Graag wil ik ook alle co-auteurs, artsen en verpleegkundigen betrokken bij de COLON studie bedanken. Jody en Henny, bedankt voor het meten van de vitamine D levels en Michiel en Nhien bedankt voor al het werk rond het meten en cleanen van de inflammatiemarkers. De dames van diëtetiek, bedankt voor het berekenen van de voedingsdata. Anne, Mechteld en Henriette bedankt voor de praktische hulp rondom de label printer, de Biobank, lab materialen and de VIP toegang.

Collega`s van de EnCoRe-studie (Eline, Annaleen, Marlou-Floor), heel erg bedankt voor de prettige samenwerking rondom het uitwisselen van de data.

Mijn collega`s van de NAD groep. Ik wil jullie allemaal bedanken voor de leuke en zinvolle discussies tijdens de MENU-D, de feedback op mijn draft papers bij de NAD-paper club en natuurlijk niet te vergeten voor de gezelligheid tijdens de koffie-uurtjes, de lunchwandelingen en het jaarlijkse uitje! Thank you!

Janita, Varisha, Laura, Lieke, Gemma, Ellen, Diana, Joanne, Meilissa, Ghazala, Floor, Lonneke, Anne-Wil, Anouk, Mirthe bedankt dat ik jullie mocht begeleiden tijdens jullie scriptie. Dit was voor mij een ontzettend leuke en ook leerzame ervaring.

Mariëlle, Liesbeth, ELLY, Anniek, Esther, bedankt voor de gezellige diners en spelletjesavonden. Ontspanning is af en toe ook nodig en jullie zijn het perfecte gezelschap daarvoor. Op naar nog een boel diners en gezellige avondjes!

Thank you office mates of 1061 (Desiree, Pol, ELLY, Gerdine, Marie-Luise, Ruoxuan, Apple, Ilse, Cong) for the pleasant work atmosphere and the nice activities outside working hours (Sinterklaas, lasergamen). Elbrich, Guadalupe, Moniek, Paulina, Rachelle, Pol, organizing the PhD tour to Canada with you was a great experience.

Liesbeth en Melanie, wat ontzettend fijn dat jullie mijn paranimfen willen zijn. Liesbeth, dank je wel voor de vele gezellige wandelingen, fietstochten, theemomentjes en dinertjes. Dankjewel ook dat je altijd klaar staat met een luisterend oor. Melanie, bedankt dat je zo`n fijne vriendin bent en bedankt voor de gezellige thee en diner momentjes. 
Dames van het secretariaat, door jullie hulp en inzet verliepen alle administratieve dingetjes altijd vlekkeloos. Jasmijn en Gea, bedankt voor jullie hulp en de gezellige praatjes in de wandelgang.

Voedingsmiepjes, Nicole, Britt \& Melanie, samen met jullie begon ik 9 jaar geleden aan de studie Voeding en Gezondheid. Bedankt voor de gezelligheid tijdens en na de studietijd! Ik hoop dat we nog lang contact blijven houden.

Papa en mama, dank jullie wel dat ik altijd weer welkom ben in het veilige, warme nest aan de Wolfersveenweg. De weekenden en later zaterdagen thuis, hoewel vaak vol met tuin en boerderij werk, waren voor mij perfect om weer op te laden en tot rust te komen. Hendrik, Regine, Annegreet en Marlien dank jullie wel voor de rumoerige weekenden thuis, die mis ik nu best nog wel eens. Dries \& Lianne, eigenlijk zien we elkaar veel te weinig. Mama, dank u voor het lezen en corrigeren van de Nederlandse samenvatting, want zoals $u$ al zei, mijn Nederlands is af en toe bedroevend.

Pa Stassen, Yvette, Robine, Ivo, Oma Annie, Stefanie, Marc-Jan, Jim, Britt, familie en vrienden bedankt voor jullie interesse in mijn promotietraject. Vooral ook bedankt voor de gezellige theetjes en dinertjes en afleiding afgelopen jaren.

Nick, mijn rots in de branding. Dank je wel dat ik bij jou helemaal mezelf kan zijn en je er altijd voor me bent en me onvoorwaardelijk steunt. Dank je wel voor je liefde, vertrouwen en geduld. Ik houd van jou! 

ABOUT THE AUTHOR 


\section{CURRICULUM VITAE}

Evertine Wesselink (Vera) was born on July 2, 1993 in Zelhem, the Netherlands. In 2012, she started with her bachelor in Nutrition and Health at Wageningen University, the Netherlands. During her bachelor, she completed a minor in Gastrointestinal diseases at the Erasmus Medical Centrum in Rotterdam, the Netherlands. After obtaining her BSC degree in 2015, she continued with the

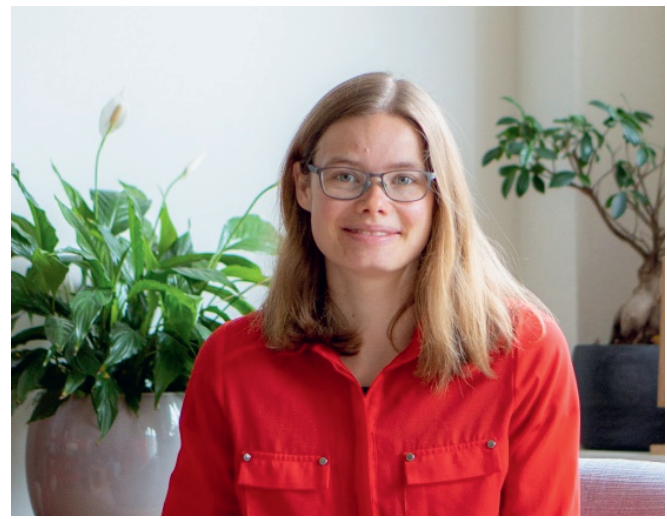
master Nutrition and Health, where she specialized in nutritional physiology and health status. For her MSc thesis, she investigated dietary magnesium and calcium intake in relation to the occurrence and severity of chemotherapy-induced peripheral neuropathy. She completed her MSc internship at Ziekenhuis Gelderse Vallei in Ede, the Netherlands. During her internship she studied the association between hyperosmolar drugs and food intake, food intolerance, gastro-intestinal problems and clinical outcomes as mortality. At the same time, she performed a literature study in which she investigated mitochondrial functioning in critically ill patients and the potential role of food components in mitochondrial functioning. After she achieved her MSc degree in 2017, she started as a PhD candidate at the division of Human Nutrition and Health at Wageningen University. During her PhD project, she investigated the associations between vitamin $\mathrm{D}$, inflammation and colorectal cancer outcomes. Results of this project are described in this thesis. Vera collected and analyzed observational data for the COLON study. She is also involved in a research project within the International Survival Analysis in Colorectal cancer Consortium (ISACC), in which she investigates calcium intake and geneenvironment interactions in relation to colorectal cancer survival. During her PhD project, Vera attended various courses and she presented her work at several conferences. She was involved in teaching and supervised many BSc and MSc students. She was part of the organizing committee of the PhD tour to East Canada in 2019. She is currently working as a postdoctoral researcher on the RegioDeal Foodvalley, in which she will continue to investigate the role of nutrition and lifestyle on cancer outcomes. Her ambition is to further unravel underlying mechanisms behind the associations between diet, lifestyle and chronic diseases by combining epidemiology and biology. She has many hobbies too, including reading, all kind of creative activities, gardening, cycling, working on the farm and enjoying time in nature. 


\section{LIST OF PUBLICATIONS}

\section{Publications in peer-reviewed journals as a first author}

Wesselink E, Balvers MGJ, Kok DE, Winkels RM, van Zutphen M, Schrauwen RWM, Keulen ETP, Kouwenhoven EA, Breukink SO, Witkamp RF, de Wilt JHW, Bours MJL, Weijenberg MP, Kampman E, van Duijnhoven FJB. Levels of inflammation markers are associated with the risk of recurrence and all-cause mortality in colorectal cancer patients. Cancer Epidemiol Biomarkers Prev. 2021; 30(6):1089-1099.

Wesselink E, Kok DE, de Wilt JHW, Bours MJL, van Zutphen M, Keulen ETP, Kruyt FM, Breukink SO, Kouwenhoven EA, van den Ouweland J, Weijenberg MP, Kampman E, van Duijnhoven FJB. Sufficient 25-Hydroxyvitamin D Levels 2 Years after Colorectal Cancer Diagnosis are Associated with a Lower Risk of All-cause Mortality. Cancer Epidemiol Biomarkers Prev. 2021 Apr;30(4):765-773.

Wesselink E, Staritsky LE, van Zutphen M, Geijsen AJMR, Kok DE, Kruyt F, Veenstra RP, Spillenaar Bilgen EJ, Kouwenhoven EA, de Wilt JHW, Kampman E, van Duijnhoven FJB. The association between the adapted dietary inflammatory index and colorectal cancer recurrence and all-cause mortality. Clin Nutr. 2021 Jan 11:S02615614(21)00010-8.

Wesselink E, van Baar H, van Zutphen M, Tibosch M, Kouwenhoven EA, Keulen ETP, E Kok D, van Halteren HK, Breukink SO, de Wilt JHW, Weijenberg MP, Kenkhuis MF, Balvers MGJ, Witkamp RF, van Duijnhoven FJB, Kampman E, Beijer S, Bours MJL, Winkels RM. Inflammation Is a Mediating Factor in the Association between Lifestyle and Fatigue in Colorectal Cancer Patients. Cancers (Basel). 2020 Dec 9;12(12):3701.

Wesselink E, Kok DE, Bours MJL, de Wilt JHW, van Baar H, van Zutphen M, Geijsen AMJR, Keulen ETP, Hansson BME, van den Ouweland J, Witkamp RF, Weijenberg MP, Kampman E, van Duijnhoven FJB. Vitamin D, magnesium, calcium, and their interaction in relation to colorectal cancer recurrence and all-cause mortality. Am J Clin Nutr. 2020;111(5):1007-1017.

Wesselink, E., Balvers, M., Bours, M., de Wilt, J., Witkamp, R. F., van Baar, H., Geijsen, A., van Halteren, H., Keulen, E., Kok, D. E., Kouwenhoven, E. A., van den Ouweland, J., van Zutphen, M., Weijenberg, M. P., Kampman, E., \& van Duijnhoven, F. (2020). The association between circulating levels of vitamin D and inflammatory markers in the first 2 years after colorectal cancer diagnosis. Therapeutic advances in gastroenterology, 13, 1756284820923922.

Wesselink E, Balvers M, Bours MJL, de Wilt JHW, Aquarius M, Breukink SO, Hansson B, Witkamp RF, van Baar H, Geijssen A, Halteren H, Keulen ETP, Kok DE, van den Ouweland J, van Roekel EH, Winkels R, Snellen M. van Zutphen M, Weijenberg MP, Kampman E, van Duijnhoven FJB. Chemotherapy and vitamin D supplement use are major determinants of circulating vitamin $D$ levels during the first six months after colorectal cancer diagnosis: a prospective cohort study. The Journal of steroid biochemistry and molecular biology. 2020;199:105577.

Wesselink E, Koekkoek WAC, Grefte S, Witkamp RF, van Zanten ARH. Feeding mitochondria: Potential role of nutritional components to improve critical illness convalescence. Clinical nutrition 2019; 38(3):982-995. 
Wesselink E, Winkels RM, van Baar H, Geijsen A, van Zutphen M, van Halteren HK, Hansson BME, Radema SA, de Wilt JHW, Kampman E, Kok DEG. Dietary Intake of Magnesium or Calcium and Chemotherapy-Induced Peripheral Neuropathy in Colorectal Cancer Patients. Nutrients 2018;10(4),398.

Wesselink E, Koekkoek K, Looijen M, van Blokland DA, Witkamp RF, van Zanten ARH. Associations of hyperosmolar medications administered via nasogastric or nasoduodenal tubes and feeding adequacy, food intolerance and gastrointestinal complications amongst critically ill patients: A retrospective study. Clinical nutrition ESPEN 2018;25: 78-86.

\section{Co-authored publications in peer-reviewed journals}

Kok DE, Arron MNN, Huibregtse T, Kruyt FM, Bac DJ, van Halteren HK, Kouwenhoven EA, Wesselink E, Winkels RM, van Zutphen M, van Duijnhoven FJB, de Wilt JHW, Kampman E. Association of Habitual Preoperative Dietary Fiber Intake With Complications after Colorectal Cancer Surgery. JAMA surgery. Published online June 16, 2021.

van Zutphen M, van Duijnhoven FJB, Wesselink E, Schrauwen RWM, Kouwenhoven EA, van Halteren HK, de Wilt JHW, Winkels RM, Kok DE, Boshuizen HC. Identification of Lifestyle Behaviors Associated with Recurrence and Survival in Colorectal Cancer Patients Using Random Survival Forests. Cancers. 2021;13(10):2442.

Geijse AJMR, Kok DE., van Zutphen M., Keski-Rahkonen P., Achaintre D., Gicquiau A., Gsur A., Kruyt F.M., Ulrich CM., Weijenberg MP., de Wilt JHW., Wesselink E., Scalbert A., Kampman E. and van Duijnhoven FJB. Diet quality indices and dietary patterns are associated with plasma metabolites in colorectal cancer patients. European journal of nutrition. 2021.

van Zutphen M, Boshuizen HC, Kenkhuis M-F, Wesselink E, Geijsen AJMR, de Wilt JHW, van Halteren HK, Spillenaar Bilgen EJ, Keulen ETP, Janssen-Heijnen MLG, Breukink SO, Bours MJL, Kok DE, Winkels RM, Weijenberg MP, Kampman E and van Duijnhoven FJB. Lifestyle after colorectal cancer diagnosis in relation to recurrence and all-cause mortality. The American journal of clinical nutrition. 2021; 113(6):1447-1457.

Koole JL, Bours MJL, Geijsen, AJMR, Gigic B, Ulvik A, Kok DE, Brezina S, Ose J, Baierl A, Böhm J, Brenner H, Breukink SO, Chang-Claude J, van Duijnhoven FJB, van Duijvendijk P, Gumpenberger T, Habermann N, van Halteren HK, Hoffmeister M, Holowatyj AN, Janssen-Heijnen MLG, Keulen ETP, Kiblawi R, Kruyt FM, Li Cl, Lin T, Midttun $\varnothing$, Peoples AR, van Roekel EH, Schneider MA, Schrotz-King P, Ulrich AB, Vickers K, Wesselink E, de Wilt JHW, Gsur A, Ueland PM, Ulrich CM, Kampman E and Weijenberg MP. Circulating B-vitamin biomarkers and Bvitamin supplement use in relation to quality of life in patients with colorectal cancer: results from the FOCUS consortium. The American journal of clinical nutrition. 2021; 113 (6):1468-1481.

Vinke P, Wesselink E, van Orten-Luiten W, van Norren K. The Use of Proton Pump Inhibitors May Increase Symptoms of Muscle Function Loss in Patients with Chronic Illnesses. Int J Mol Sci. 2020;21(1):323.

van Baar, H., Winkels, R. M., Brouwer, J. G. M., Posthuma, L., Bours, M. J. L., Weijenberg, M. P., Boshuizen, H. C., van Zutphen, M., van Duijnhoven, F. J. B., Kok, D. E., Wesselink, E., Slooter, G. D., Spillenaar Bilgen, E. J., Hansson, B. M. E., de Wilt, J. H. W., Kampman, E. and Beijer, S. Associations of Abdominal Skeletal Muscle Mass, Fat Mass, and Mortality among Men and Women with Stage I-III Colorectal Cancer. Cancer epidemiology, biomarkers \& prevention : a publication of the American Association for Cancer Research, cosponsored by the American Society of Preventive Oncology. 2020;29(5):956-965.

van Baar, H., Bours, M. J. L., Beijer, S., van Zutphen, M., van Duijnhoven, F. J. B., Kok, D. E., Wesselink, E., de Wilt, J. H. W., Kampman, E. and Winkels, R. M. Body composition and its association with fatigue in the first 2 years after colorectal cancer diagnosis. Journal of Cancer Survivorship. 2020. 
Geijsen A, van Roekel EH, van Duijnhoven FJB, Achaintre D, Bachleitner-Hofmann T, Baierl A, Bergmann MM., Boehm J., Bours MJL., Brenner H., Breukink SO., Brezina S., Chang-Claude J., Herpel E., de Wilt JHW., Gicquiau A., Gigic B., Gumpenberger T., Hansson BME., Hoffmeister M., Holowatyj AN., Karner-Hanusch J., Keski-Rahkonen P., Keulen ETP., Koole JL., Leeb G, Ose J., Schirmacher P., Schneider MA., Schrotz-King P., Stift A., Ulvik A., Vogelaar FJ., Wesselink E., van Zutphen M., Gsur A., Habermann N., Kampman E., Scalbert A., Ueland PM., Ulrich AB., Ulrich CM., Weijenberg MP. and Kok DE. Plasma metabolites associated with colorectal cancer stage: Findings from an international consortium. International journal of cancer Journal international of cancer. 2020; 146(12):3256-3266.

Geijsen AJMR, Ulvik A, Gigic B, Kok DE, van Duijnhoven FJB, Holowatyj AN, Brezina S, van Roekel EH, Baierl A, Bergmann MM, Böhm J, Bours MJL, Brenner H, Breukink SO, Bronner MP, Chang-Claude J, de Wilt JHW, Grady WM, Grünberger T, Gumpenberger T, Herpel E, Hoffmeister M, Huang LC, Jedrzkiewicz JD, Keulen ETP, Kiblawi R, Kölsch T, Koole JL, Kosma K, Kouwenhoven EA, Kruyt FM, Kvalheim G, Li Cl, Lin T, Ose J, Pickron TB, Scaife CL, Schirmacher P, Schneider MA, Schrotz-King P, Singer MC, Swanson ER, van Duijvendijk $P$, van Halteren HK, van Zutphen M, Vickers K, Vogelaar FJ, Wesselink E, Habermann N, Ulrich AB, Ueland PM, Weijenberg MP, Gsur A, Ulrich CM, Kampman E. Circulating Folate and Folic Acid Concentrations With Colorectal Cancer Recurrence and Survival. JNCI Cancer Spectr. 2020;4(5):pkaa051.

Winkels RM, Van Brakel L, Van Baar H, Beelman RB, Van Duijnhoven FJB, Geijsen A, Van Halteren HK, Hansson BME, Richie JP and Sun D, Wesselink E, van Zutphen M, Kampman E and Kok DE. Are Ergothioneine Levels in Blood Associated with Chronic Peripheral Neuropathy in Colorectal Cancer Patients Who Underwent Chemotherapy? Nutrition and cancer. 2020;72(3):451-459.

van Zutphen M, Boshuizen HC., Kok DE., van Baar H, Geijsen AJMR., Wesselink E, Winkels RM., van Halteren HK., de Wilt JHW., Kampman E and van Duijnhoven FJB. Colorectal cancer survivors only marginally change their overall lifestyle in the first 2 years following diagnosis. Journal of Cancer Survivorship. 2019; 13(6):956-967

van Zutphen M, Geelen A, Boshuizen HC, Winkels RM, Geijsen A, Wesselink E, Snellen M., Kok DE., de Wilt JHW., van de Meeberg PC., Kouwenhoven EA., van Halteren HK., Spillenaar Bilgen EJ., Kampman E. and van Duijnhoven FJB. Pre-to-post diagnosis weight trajectories in colorectal cancer patients with non-metastatic disease.

Supportive care in cancer : official journal of the Multinational Association of Supportive Care in Cancer. 2019; 27(4):1541-1549. 


\section{OVERVIEW OF COMPLETED TRAINING ACTIVITIES}

\begin{tabular}{|c|c|c|}
\hline Discipline specific activities & Organiser and location & Year \\
\hline \multicolumn{3}{|l|}{ Courses } \\
\hline Infections, immunology and tolerance & LUMC, Leiden, NL & 2018 \\
\hline $\begin{array}{l}\text { Exposure Assessment in Nutritional } \\
\text { Research }\end{array}$ & VLAG, Wageningen, NL & 2018 \\
\hline $\begin{array}{l}\text { Masterclass "Nutrition and Cancer: } \\
\text { from Bench to Bed to Behaviour" }\end{array}$ & $\begin{array}{l}\text { VLAG/WUR Division of Human } \\
\text { Nutrition \& Health, Wageningen, } \\
\text { NL }\end{array}$ & 2019 \\
\hline ENLP essential program & ENLP, online & 2021 \\
\hline \multicolumn{3}{|l|}{ Conferences and meetings } \\
\hline WEON 2018 "Science for Society" & RIVM, Bilthoven, NL & 2018 \\
\hline Dutch Nutritional Science Days & NAV, Heeze, NL & 2018 \\
\hline Dutch Nutrional Science Days & NAV, Heeze, NL & 2019 \\
\hline Nutrition live online 2020 & ASN, online & 2020 \\
\hline $\begin{array}{l}\text { Symposium Lifestyle \& Cancer - } \\
\text { Evidence Matters }\end{array}$ & AICR, online & 2021 \\
\hline $5^{\text {th }}$ International Molecular & Dana-Farber Cancer Institute & 2021 \\
\hline $\begin{array}{l}\text { Pathological Epidemiology meeting } \\
\text { WEON } 2021 \text { "Science in an online } \\
\text { society" }\end{array}$ & $\begin{array}{l}\text { Boston, online } \\
\text { UMCA, online }\end{array}$ & 2021 \\
\hline \multicolumn{3}{|l|}{ General courses and activities } \\
\hline Presenting with impact & WGS, Wageningen, NL & 2017 \\
\hline $\begin{array}{l}\text { Concepts and Methods in } \\
\text { Epidemiology }\end{array}$ & WUR, Wageningen, NL & 2018 \\
\hline PhD week & VLAG, Wageningen, NL & 2017 \\
\hline Introduction to $\mathrm{R}$ & VLAG, Wageningen, NL & 2018 \\
\hline PhD Carousel 2018 & WGS, Wageningen, NL & 2018 \\
\hline Supervising BSc and MSc students & VLAG, Wageningen, NL & 2019 \\
\hline Pitch Training & $\begin{array}{l}\text { WUR Division of Human Nutrition } \\
\text { \& Health, Wageningen, NL }\end{array}$ & 2019 \\
\hline Course Good Clinical Practice & $\begin{array}{l}\text { WUR Division of Human Nutrition } \\
\& \text { Health, Wageningen, NL }\end{array}$ & 2020 \\
\hline Critical thinking and argumentation & WGS, online & 2020 \\
\hline Scientific Writing & WGS, online & 2020 \\
\hline Writing grant proposals & WGS, online & 2021 \\
\hline $\begin{array}{l}\text { Workshop Hoe blijf ik in beeld in een } \\
\text { online wereld }\end{array}$ & NAV, online & 2020 \\
\hline $\begin{array}{l}\text { Short Course Mendelian } \\
\text { Randomization }\end{array}$ & Imperial College Londen, online & 2021 \\
\hline
\end{tabular}


Optional courses and activities

Preparation of research proposal

WUR, Wageningen, NL

2017

Participation and organizing PhD study

WUR, Canada

2019

tour Canada

Staff seminars and chair group

WUR, Wageningen, NL

2017-2021

meetings

Wetenschapsavonden Alliantie

Alliantie Voeding in de Zorg, NL

2018-2020

Voeding in de Zorg 


\section{COLOPHON}

The research described in this thesis was financially supported by Wereld Kanker Onderzoek Fonds, including funds from grant 2014/1179 as part of the World Cancer Research Fund International Regular Grant Programme; Alpe d'Huzes/Dutch Cancer Society (UM-2010-4867, UM 2012-5653, UW 2013-5927, UW 2015-7946); and ERA-NET on Translational Cancer Research (TRANSCAN/Dutch Cancer Society (UW2013-6397, UW2014-6877) and the Netherlands Organization for Health Research and Development (ZonMw), the Netherlands) and by a grant from Kankeronderzoekfonds Limburg as part of Health Foundation Limburg (Grant No. 00005739).

Financial support from Wageningen University for printing this thesis is gratefully acknowledged.

Cover design by Evertine Wesselink

Layout by Evertine Wesselink

Printed by Proefschriftmaken.nl

Copyright (C) Evertine Wesselink, 2021 

